University of Nebraska - Lincoln DigitalCommons@University of Nebraska - Lincoln

Architectural Engineering -- Dissertations and

Student Research

Architectural Engineering

$4-2012$

THE ASSESSMENT OF HIGH DYNAMIC RANGE LUMINANCE MEASUREMENTS WITH LED LIGHTING

Yulia I. Tyukhova

University of Nebraska - Lincoln, jtyukhova@yahoo.com

Follow this and additional works at: http://digitalcommons.unl.edu/archengdiss

Part of the Architectural Engineering Commons

Tyukhova, Yulia I., "THE ASSESSMENT OF HIGH DYNAMIC RANGE LUMINANCE MEASUREMENTS WITH LED

LIGHTING" (2012). Architectural Engineering -- Dissertations and Student Research. 17.

http://digitalcommons.unl.edu/archengdiss/17

This Article is brought to you for free and open access by the Architectural Engineering at DigitalCommons@University of Nebraska - Lincoln. It has been accepted for inclusion in Architectural Engineering -- Dissertations and Student Research by an authorized administrator of

DigitalCommons@University of Nebraska - Lincoln. 
THE ASSESSMENT OF HIGH DYNAMIC RANGE LUMINANCE

MEASUREMENTS WITH LED LIGHTING

by

Yulia I. Tyukhova

\section{A THESIS}

Presented to the Faculty of

The Graduate College at the University of Nebraska

In Partial Fulfillment of Requirements

For the Degree of Master of Science

Major: Architectural Engineering

Under the Supervision of Professor Clarence Waters

Lincoln, Nebraska

April, 2012 


\title{
THE ASSESSMENT OF HIGH DYNAMIC RANGE LUMINANCE MEASUREMENTS WITH LED LIGHTING
}

\author{
Yulia I. Tyukhova, M.S.
}

University of Nebraska, 2012

Adviser: Clarence Waters

This research investigates whether a High Dynamic Range Imaging (HDRI) technique can accurately capture luminance values of a single LED chip. Previous studies show that a digital camera with exposure capability can be used as a luminance mapping tool in a wide range of luminance values with an accuracy of $10 \%$. Previous work has also demonstrated the ability of HDRI to capture a rapidlychanging lighting environment with the sun. However, these publications don't investigate HDRI's ability to capture a bright light source with a narrow light distribution (LED lighting).

Some of the existing concerns in LED technology today include low quality products on the market, inaccurate performance claims, and insufficient information on Solid-State Lighting (SSL) products. Division 2 in the International Commission on Illumination (CIE) (Physical Measurement of Light and Radiation) prepares the technical report (TC2-58) on measuring LED radiance and luminance; however, progress has not yet been published. Manufacturers do not provide luminance data on their products even though luminance is the most important quantity in lighting design and illuminating engineering. It is one of the direct stimuli to vision, and many measures of performance and perception. 
In this research two conventional luminance measurement methods of a single LED chip are implemented. One method involves the use of a luminance meter with a close-up lens, and the other method allows obtaining luminance through calculations from the illuminance measurements. Luminous intensity data can be determined using direct illuminance measurements taken in a created photometer. These data along with dimensions of an LED can then be used to calculate average luminance.

Varying apertures and shutter speeds in a digital camera allows obtaining a sequence of images with different exposures. These images are combined together using software to create an HDRI that gives pixel by pixel luminance values. The HDRI of a single LED chip is obtained using a neutral density filter. The results of this research indicate that the HDRI technique can capture luminance values of a single LED chip. 


\section{AUTHOR'S ACKNOWLEDGMENTS}

First and foremost, I would like to express my utmost gratitude to my adviser

Dr. Waters for all his guidance and encouragement. Dr. Waters has inspired me with the topic for this work, gave me confidence and provided lots of support throughout the research. I appreciate all his help and I feel really lucky to have him as an adviser.

I would like to thank GE Lighting for providing LED luminaires for this research, in particular Jason Brown for all his assistance.

I appreciate all the help that I have received from HDRI forum. But especially I would like to thank Greg Ward for his great help and fast responses.

I wish to thank Robert Guglielmetti, Jennifer Scheib, and Dr. Tiller for willingness to help, their time, and valuable advice. Also, I would like to thank them and Dr. Alahmad for being members of my committee.

And finally, I would like to thank my family for all the love and support that I receive from them. 


\section{Corrigenda}

September 2012

The following corrections have been made to the text:

Page $72\left(19.44 * 10^{6} \mathrm{~cd} / \mathrm{m}^{2}\right)$

Page $74\left(19.44 * 10^{6} \mathrm{~cd} / \mathrm{m}^{2}\right)$

Page $80\left(19.44 * 10^{6} \mathrm{~cd} / \mathrm{m}^{2}\right)$

Page 127 twice $\left(19.44 * 10^{\mathbf{6}} \mathbf{c d} / \mathbf{m}^{2}\right)$

Page $130\left(\mathbf{1 9 . 4 4} * 10^{6} \mathbf{c d} / \mathbf{m}^{2}\right)$ replaces $\quad\left(19.44 * 10^{7} \mathrm{~cd} / \mathrm{m}^{2}\right)$

66

c6 c 


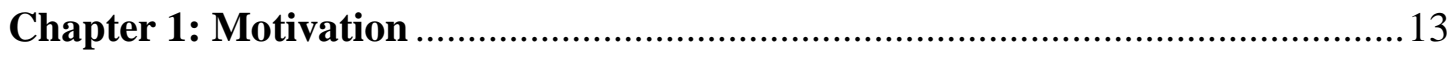

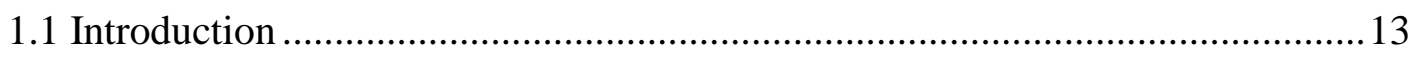

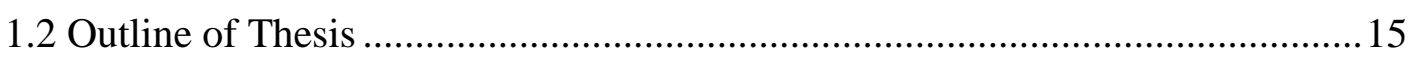

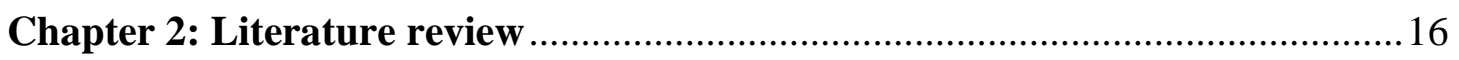

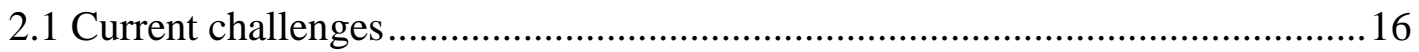

2.2 Theory of High Dynamic Range Imaging with the application in Lighting ......23

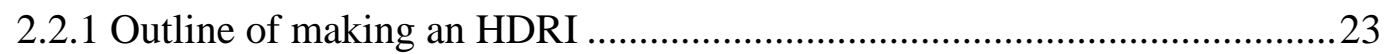

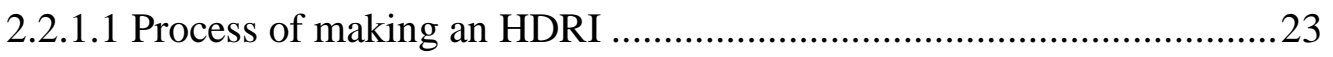

2.2.1.2 Practical guidelines of making an HDRI .........................................26

2.2.2. Response curve of a specific camera and lens combination......................29

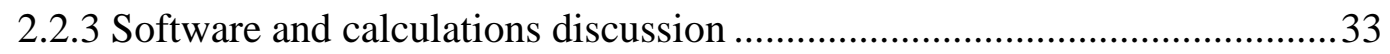

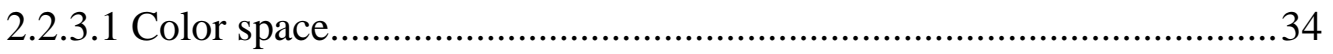

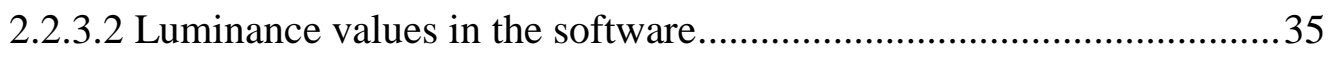

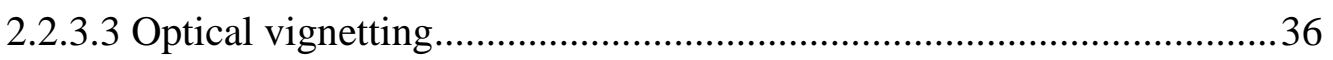

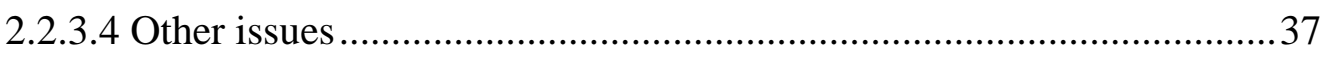

2. 3 Studies of HDRI technique validation for lighting measurements ...................39

2.3.1 Studies of the HDRI technique validation in a scene with no bright light

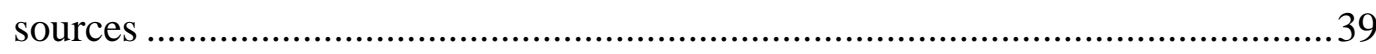

2.3.2 Studies of the HDRI technique capturing a natural scene and/or the sun ...51

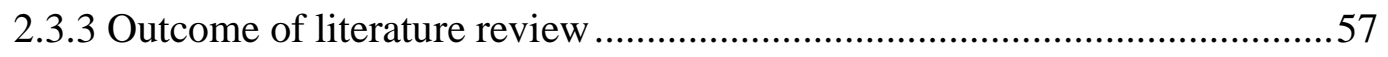

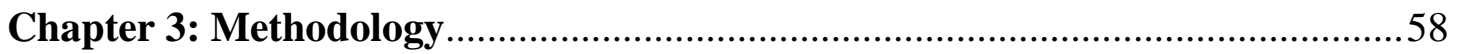

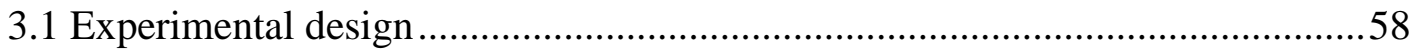

3.2 Measurements of luminance of a single LED chip with traditional methods ....60

3.2.1 Measurements of a single LED chip's dimensions ..................................60

3.2.2 Measuring luminance of a single LED chip in GE garage fixture with the

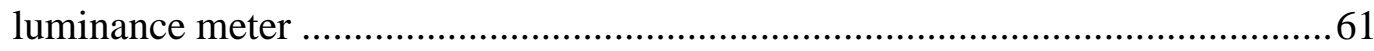

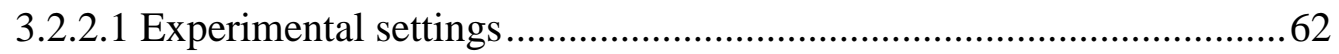

3.2.2.2 Analyzing the overlay of a single LED chip area and the measuring

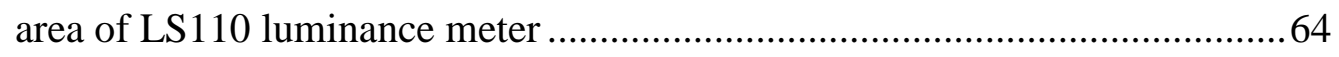

3.2.3 Luminance calculation through the luminous intensity curve and area

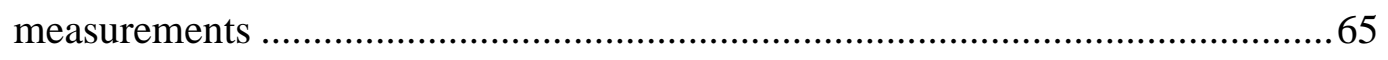

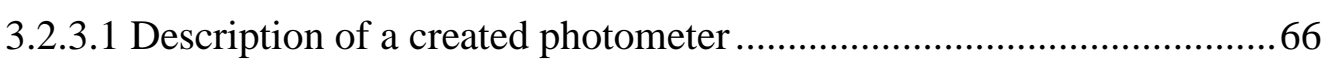

3.2.3.2 Analysis of direct illuminance measurements from a single LED chip 
3.3 The implementation of the HDRI technique for lighting measurements ..... .80

3.3.1 Response curves for cameras and lenses .80

3.3.2 Optical vignetting .83

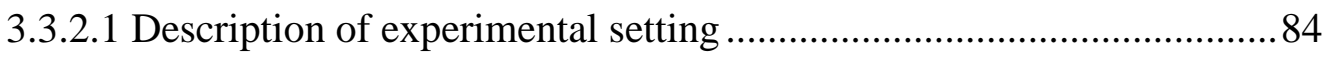

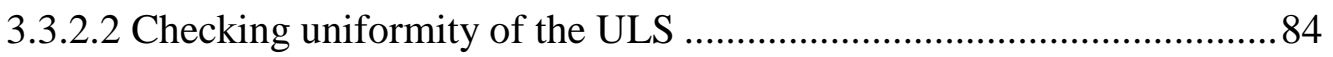

3.3.2.3 Build-in peripheral illumination correction experiment ......................86

3.3.2.4 Optical vignetting effect with different zoom settings .89

3.3.3 Defining the experimental settings for capturing an HDRI of a single LED chip in GE garage fixture

3.3.3.1. Capture of a single LED chip trial ................................................... 92

3.3.3.2 Analysis of a scene used for obtaining the response curve.................94

3.3.3.3 Scene with a wider dynamic range ................................................95

3.3.3.4. Exposures analysis with the histograms in Photoshop CS5 ...............96

3.4 Measurements of a single LED chip in GE garage fixture with the HDRI technique

3.4.1 Response curve for Canon EOS 7D fitted with 28-105 mm CANON zoom lens

3.4.2 Using raw2hdr Perl script for fusing a sequence of images ..................... 101

3.4.3 The HDRI capture of an incandescent light source ..................................102

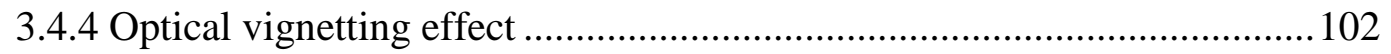

3.4.4.1 Comparing optical vignetting effect experiment results for two software

3.4.4.2 Analysis of the critical area of the HDRI where LED and reflectance standard's calibration zone are located 106

3.4.5 Capturing a single LED chip with the ND filter in RAW format and fusing images with raw2hdr Perl script ................................................................. 110

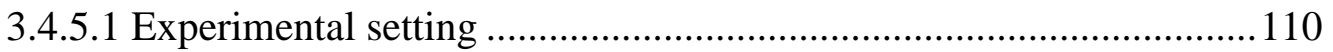

3.4.5.2 Analysis of average luminance measurements with the HDRI .........114

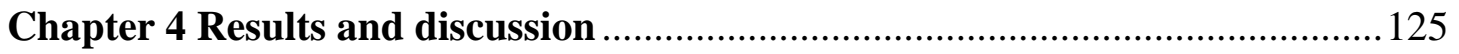

4.1 Discussion of results and recommendations ................................................. 125

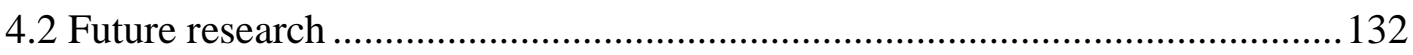

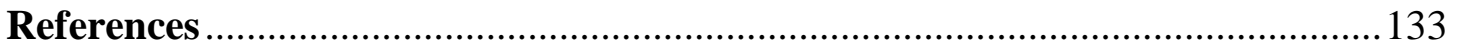

Appendix A: Table of experimental settings from studies of HDRI in lighting ...... 140 Appendix B: Specification of equipment. 
Appendix C: LED output measurements depending on line voltage over the day-time

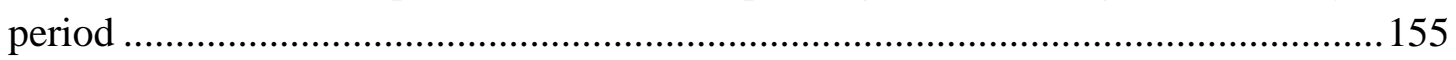

Appendix D: Excerpt from HDRI Mailing Lists (www.radiance-online.org)........... 156

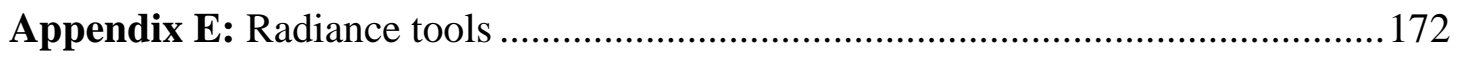

(http://www.radiance-online.org/pipermail/hdri/2012-February/000363.html) ........ 172

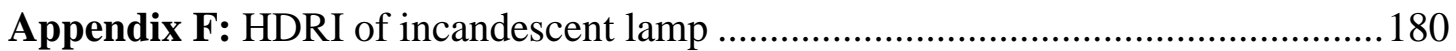




\section{LIST OF FIGURES}

Figure 2-1. Response functions of a few popular films and video cameras provided by their manufacturers (Mitsunaga et al. 1999)

Figure 2-2. Response functions of a few popular cameras (according webHDR

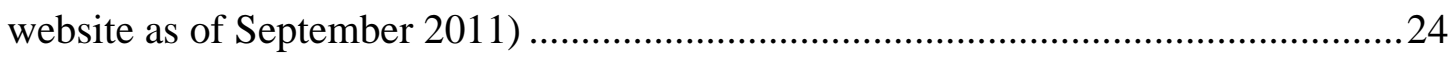

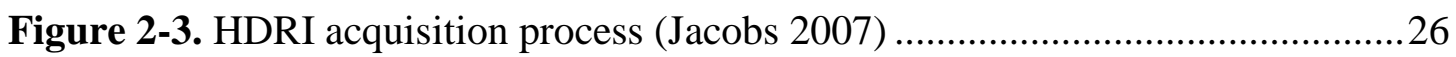

Figure 2-4. Relationship between image irradiance $\mathrm{E}$ and scene radiance $\mathrm{L}$ ("Introduction to Computer Vision" as of October 2011) ..........................................29

Figure 2-5. Recovering camera's response curve.

Figure 2-6. Images of flare components of a point light source at different locations (Xiao et al. 2002) .38

Figure 2-7. A- Munsell chip, B - Experiment set-up (Anaokar et al. 2005)

Figure 2-8. X-Rite ColorChecker chart that was used in (Chung et al. 2010) to analyze variations of calibration factor over time for the HDR photography in a daylit interior scene.

Figure 2-9. "Digital filter" for compensating luminance loss due to the vignetting effect in the study (Chung et al. 2010).....

Figure 2-10. Defining solid angles of central pixel $\mathrm{d} \omega_{\mathrm{c}}$, and any other pixel i on a sensor $\mathrm{d} \omega_{\mathrm{i}}$ (Moeck et al. 2006)

Figure 2-11. Set-up for assessing accuracy of obtaining illuminance values from the luminance map

Figure 2-12. A typical HDR sequence spanning the 17 stops of the sky and the sun in

7 exposures

Figure 2-13. Fisheye projection and application of the HDR image as a light source in a computer simulation (Inanici 2010)

Figure 2-14. a - Mean luminance of the scene, $b$ - Predetermined absolute luminance threshold area, c-Mean luminance task area (desk and monitor), d - Mean luminance task area (defined as subtended solid angle encompassing the screen and keyboard) 56

Figure 3-1. Cree ${ }^{\circledR}$ XLamp ${ }^{\circledR}$ XP-E LEDs 61

Figure 3-2. XP-E LED dimensions (CREE LED lighting)

Figure 3-3. Minimum measured diameter of an object with a luminance meter LS110 and the close-up lens \#135 at a minimum distance.

Figure 3-4. The set-up for the luminance meter measurement of a single LED chip.63

Figure 3-5. Metering circle of LS1 10 luminance meter and the area of a single LED chip. Relative sizes are to scale

Figure 3-6. Created photometer for measuring direct illuminance from a light source

Figure 3-7. Tube covering the illuminance meter..................................................6 68

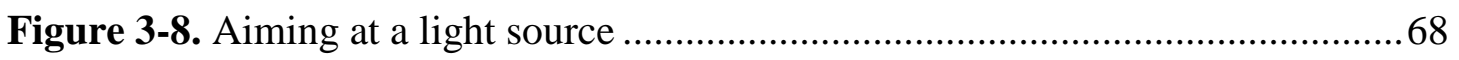

Figure 3-9. GE Garage Luminaire without the cover and reflectors ..........................69

Figure 3-10. Dimensions of the created cardboard tube ............................................ 70

Figure 3-11. Changing gear in 2.5 degree increments .......................................... 70 
Figure 3-12. Normal to the LED array. .71

Figure 3-13. Luminous intensity curve of a single LED chip, cd ............................. 75

Figure 3-14. Calculated luminance values of a single LED chip depending on the angle, $\mathrm{cd} / \mathrm{m}^{2}$.

Figure 3-15. Specification of the axes over the scanned manufacturer's data on luminous intensity curve (GetData Graph Digitizier software) .77

Figure 3-16. Obtaining data from the luminous intensity curve with the point capture mode (GetData Graph Digitizier software)

Figure 3-17. Digitalized manufacturer's and measured/calculated intensity curves of a single LED chip, relative units .79

Figure 3-18. Experimental set-up for deriving the response curve ............................ 81

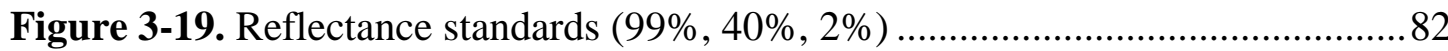

Figure 3-20. Experimental set-up for evaluating optical vignetting effect .................84

Figure 3-21. Five points of ULS of an exit port where luminance values are measured

Figure 3-22. HDRI of ULS with peripheral Illumination correction on (CANON EOS 7D with zoom lens 16-35 mm)

Figure 3-23. HDRI of ULS and the analyzed areas

Figure 3-24. HDRI of ULS with peripheral Illumination correction off (CANON EOS $7 \mathrm{D}$ with zoom lens $16-35 \mathrm{~mm}$ ) .88

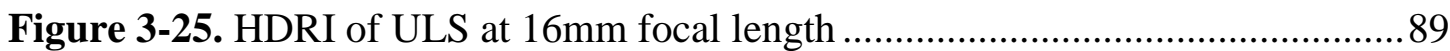

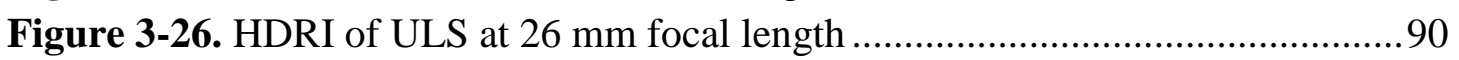

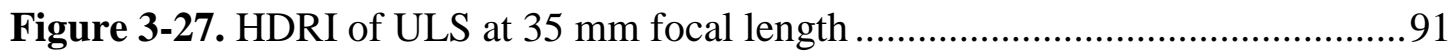

Figure 3-28. Experimental setting for obtaining an HDRI of the LED ..................... 92

Figure 3-29. Pseudo colors HDRI of a single LED chip (obtained with CANON EOS $7 \mathrm{D}$ at $16 \mathrm{~mm}$, normal to the LED)

Figure 3-30. Statistical data for a single LED in Photosphere

Figure 3-31. Pseudo colors HDRI of camera calibration scene for obtaining RC for EOS 7D camera fitted with CANON zoom lens $16-35 \mathrm{~mm}$

Figure 3-32. Calibration scene with a wider dynamic range for obtaining the RC of camera/lens combination

Figure 3-33. Histogram of the RGB values in the shortest exposure (1/8000') of the LED sequence

Figure 3-34. Enlarged histogram of the RGB values in the shortest exposure (1/8000') of the LED sequence

Figure 3-35. Histogram of the RGB values in the longest exposure (4' ') of the LED sequence

Figure 3-36. Enlarged histogram of the RGB values in the longest exposure (4') of the LED sequence

Figure 3-37. Experimental set-up for deriving the response curve for EOS 7D and 28-

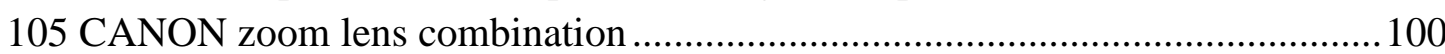

Figure 3-38. Nine areas (300x280 pixels) used for the vignetting analysis.............. 103

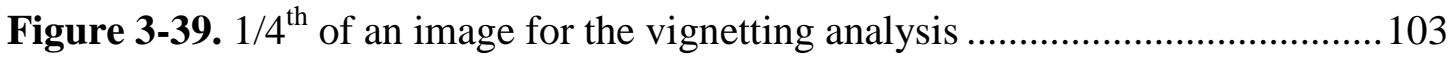


Figure 3-40. Mean luminance values and standard deviations for 9 zones of four HDRIs (two are fused in Photosphere and - two in raw2hdr)..

Figure 3-41. Part of the image with locations of the LED and reflectance standard's calibration zone 106

Figure 3-42. HDRI of ULS with the area of the LED and reflectance standard's calibration zone's locations (in pseudo colors)...

Figure 3-43. Mean luminance values and standard deviations for 9 zones of four HDRIs of the LED and reflectance standard calibration zone's location.....

Figure 3-44. The LED location on the image testing optical vignetting effect (aperture 4.5, fused in raw2hdr @ $105 \mathrm{~mm}$ ).....

Figure 3-45. Reflectance standard's calibration zone location on the image testing optical vignetting effect (aperture 4.5, fused in raw2hdr @ 105mm).....

Figure 3-46. The LED location on the image testing optical vignetting effect (aperture 16, fused in raw2hdr@105mm).

Figure 3-47. Reflectance standard's calibration zone location on the image testing optical vignetting effect (aperture 16, fused in raw2hdr @ 105mm).

Figure 3-48. Experimental setting for making an HDRI of a single LED chip ........ 111

Figure 3-49. HDR image of a single LED chip and reflectance standard ( $\rho=99 \%)$.

Calibration at white reflectance standard

Figure 3-50. HDR image of a single LED chip and reflectance standard $(\rho=99 \%)$ in pseudo colors

Figure 3-51. Histogram of the RGB values of the shortest exposure $(1 / 8000$ ', f/16, ND NDA2-703-002) in a single LED sequence

Figure 3-52. Enlarged histogram of the RGB values in the shortest exposure (1/8000', f/16, ND NDA2-703-002) in a single LED sequence.

Figure 3-53. Histogram of the RGB values in the longest exposure (30', f/4.5, ND

NDA2-703-002) in a single LED sequence

Figure 3-54. Enlarged histogram of the RGB values in the longest exposure (30', $\mathrm{f} / 4.5$, ND NDA2-703-002) in a single LED sequence

Figure 3-55. Selected area (39x37 pixels) is smaller than the LED chip (1.4 $\mathrm{mm} \times 1.4$ mm, 48 pixels x 48 pixels). Mean value $2.04 * 10^{7} \mathrm{~cd} / \mathrm{m}^{2}$.

Figure 3-56A. Selected area $(51 \times 49$ pixels) is moved around the LED chip. Mean value $9.32 * 10^{6} \mathrm{~cd} / \mathrm{m}^{2}$

Figure 3-56B. Selected area (51x49 pixels) is moved around the LED chip. Mean value $9.85 * 10^{6} \mathrm{~cd} / \mathrm{m}^{2}$

Figure 3-56C. Selected area $(51 \times 49$ pixels $)$ is moved around the LED chip. Mean value $7.46 * 10^{6} \mathrm{~cd} / \mathrm{m}^{2}$

Figure 3-56D. Selected area (51x49 pixels) is moved around the LED chip. Mean value $8.04 * 10^{6} \mathrm{~cd} / \mathrm{m}^{2}$

Figure 3-56E. Selected area (51x49 pixels) is moved around the LED chip. Mean value $8.17 * 10^{6} \mathrm{~cd} / \mathrm{m}^{2}$

Figure 3-57A. The non-uniformity of a single LED chip ...................................... 118

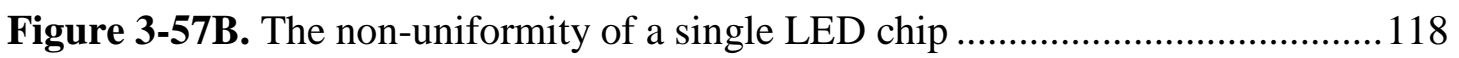

Figure 3-57C. The non-uniformity of a single LED chip 119 
Figure 3-57D. The non-uniformity of a single LED chip

Figure 3-58. Selected area of the LED chip (48x48 pixels) in pseudo colors

Figure 3-59. Cropped image of a single LED chip in pseudo colors ( 48 pixels by 48 pixels).

Figure 3-60. Luminance values obtained with the pvalue Radiance tool from a cropped HDRI of a single LED (48 pixels by 48 pixels)

Figure 3-61. X coordinate on the cropped HDRI of a single LED (pseudo colors) 122

Figure 3-62. Frequency distribution of luminance values within a single LED chip obtained with the pvalue Radiance tool from a cropped HDRI.

Figure 4-1. Calibrating an image in the zone where flare doesn't affect the image .128 Figure 4-2. Frequency distribution of luminances within a single LED chip obtained from the HDRI; mean luminance from the HDRI; and average value from illuminance measurements/calculations 131

Figure B. Calibration certificate for LS110 luminance meter 154

Figure F1. Experimental setting for making HDRI of the incandescent light source

Figure F2. HDRI of incandescent light source captured with CANON EOS 7D fitted with $28-105 \mathrm{~mm}$ zoom lens at $28 \mathrm{~mm}$

Figure F3. HDRI (pseudo colors) of incandescent light source captured with CANON EOS 7D fitted with $28-105 \mathrm{~mm}$ zoom lens at $28 \mathrm{~mm}$.

Figure F4. Enlarged HDRI of incandescent light source captured with CANON EOS

7D fitted with $28-105 \mathrm{~mm}$ zoom lens at $28 \mathrm{~mm}$

Figure F5. Enlarged HDRI (pseudo colors) of incandescent light source captured with CANON EOS 7D fitted with $28-105 \mathrm{~mm}$ zoom lens at $28 \mathrm{~mm}$ 182

Figure F6. HDRI of incandescent light source captured with CANON EOS 7D fitted with $28-105 \mathrm{~mm}$ zoom lens at $105 \mathrm{~mm}$

Figure F7. HDRI (pseudo colors) of incandescent light source captured with CANON EOS 7D fitted with 28-105mm zoom lens at $105 \mathrm{~mm}$.

Figure F8. Enlarged HDRI of incandescent light source captured with CANON EOS

7D fitted with $28-105 \mathrm{~mm}$ zoom lens at $105 \mathrm{~mm}$ 184

Figure F9. Enlarged HDRI (pseudo colors) of incandescent light source captured with CANON EOS 7D fitted with 28-105mm zoom lens at $105 \mathrm{~mm}$ 184 


\subsection{Introduction}

\section{Chapter 1: Motivation}

Light-Emitting Diode (LED) lighting is already widely used in many applications and locations. Colored lighting is used in theatres, stage productions, restaurants, casinos, and white lighting - in road and tunnel applications, sports and arenas, street and area, landscape and garage lighting. Among existing concerns in LED technology today are low quality products on the market, inaccurate performance claims, and insufficient information on Solid-State Lighting (SSL) products (Ohno 2012). Therefore, ways to characterize and measure LED products become very important.

Standards not only help improve the performance of LEDs but make comparisons among the products easier. Some lighting measuring standards for LEDs governing safety and performance are available today (Ohno 2012). Among standards for LED lighting measurements and performance are G-2-10, LM-79-08, LM-80-08, TM-16-05, and TM-21-11 developed by the Illuminating Engineering Society of North America (IES), and 127-2007 and 177-2007 developed by the International Commission on Illumination (CIE). None of the standards mentioned above dictates the procedure for obtaining luminance values of LEDs. Division 2 in CIE (Physical Measurement of Light and Radiation) prepares the technical report (TC2-58) on measuring LED radiance and luminance (according to CIE website as of February 2012), however progress has not yet been published. Manufacturers do not provide luminance data on their products, since no standard developed for LED luminance measurements exist.

Luminance is the most important quantity in lighting design and illuminating engineering. "Luminance is a measure of the light emitting power of a surface, in a particular direction, per unit apparent area" (DiLaura 2011 et al., p. 5.14). It is one of 
the direct stimuli to vision, and many measures of performance and perception (luminance of the source and background in glare analysis, luminance contrast for characterizing the task, etc.). LED is a very bright light source that introduces the problem of glare. The assessment of glare implies knowing the luminance value (DiLaura et al. 2011). Luminance of LED products must be measured in a lighting environment, and for the goal of obtaining luminance different approaches have to be considered.

There are three traditional ways to measure luminance. In one method, luminance is measured with a conventional luminance meter. In the second one, it is derived through the illuminance measurement and subsequent calculations. And in the third method, luminance is obtained through digital imaging photometer measurement.

High Dynamic Range Imaging (HDRI) technique introduces a new approach of capturing luminance values in a lighting environment. It can store the information of a scene with the range of many orders of magnitude, and values can be photometrically correct. Instead of the exhausting procedure of point by point measurements of light levels with a luminance meter like Minolta LS110, HDRI introduces the unique tool of getting thorough high resolution information about the existing lighting conditions in a fast and efficient way.

The goal of this research is to investigate whether a bright light source with a narrow light distribution (LED) can be accurately captured by the HDRI. The results of this research will contribute to the current state of knowledge on how HDRI technique can be applied for lighting measurements and analysis (e.g. glare assessment). Although there are some inaccuracies in the LEDs specification, LED 
lighting is quickly spreading on the market. The application of the HDRI technique to LED lighting will help measure, characterize, and specify LED products.

\subsection{Outline of Thesis}

This thesis investigates whether the HDRI technique can accurately capture luminance of a single LED chip. Chapter 2 provides background information on current research, covers the theory of the HDRI technique as well as practical guidelines for the successful acquisition of an HDR image. The chapter discusses previous research on the implementation of the HDRI technique to lighting measurements for both scenes with natural lighting and/or the sun and without. Chapter 3 describes the methodology of experiments conducted as well as experimental results. Luminance measurements are conducted using two traditional methods. One method involves the use of a luminance meter and a close-up lens. In the second method, average luminance is calculated from direct illuminance measurements in a created photometer. A detailed description of how the HDRI technique is implemented to capture a single LED chip is then given. The information on deriving the response curves for the camera/lens combination, optical vignetting effect assessments and fusing an HDR image in raw2hdr Perl script from a sequence of RAW images is provided. Finally, Chapter 4 discusses the results of these experiments and proposes areas for future work. 


\section{Chapter 2: Literature review}

This chapter discusses current challenges in LED products' measurements. It covers the theory of the HDRI technique implemented for lighting measurements in detail and provides practical guidelines for the successful acquisition of an HDR image. This chapter addresses previous research on the following topics: (1) HDRI with the application to lighting measurements without a bright light source in the scene, and (2) capturing lighting environment of natural scenes and/or the sun with the HDRI technique.

\subsection{Current challenges}

LED lighting is already widely used in many applications and locations. Colored lighting is used in theatres, stage productions, restaurants, casinos, and white lighting - in road and tunnel applications, sports and arena, street and area, landscape and garage lighting (Weinert 2010). Among existing concerns in LED technology today are low quality products in the market, inaccurate performance claims, and insufficient information on SSL products. Therefore, ways to characterize and measure LED products become very important. Standards help improve the performance of LEDs as well as compare products.

Further development of LEDs standards and testing procedures will allow the improvement in implementation of the technology.

Some lighting measuring standards governing safety and performance for LEDs are available today (Ohno 2012). Among standards for LEDs' lighting measurements and performance are the following:

Developed by the Illuminating Engineering Society of North America:

- $\quad$ G-2-10 Guideline for the Application of General Illumination ("White") LED Technologies (IES 2010); 
- $\quad$ LM-79-08 Approved Method: Electrical and Photometric Testing of Solid-State Lighting Devices (IES 2008);

- $\quad$ LM-80-08 Approved Method: Measuring Lumen Depreciation of LED Light Sources (IES 2008);

- $\quad$ TM-16-05 Technical Memorandum Light Emitting Diode (LED) Sources and Systems (IES 2005);

- TM-21-11 Projecting Long Term Lumen Maintenance of LED Light Sources (IES 2011).

Developed by the International Commission on Illumination:

- 127-2007 Measurement of LEDs (spectrum, luminous flux and luminous intensity curve for individual low-power LED packages (chips)) (CIE 2007);

- 177-2007 Color Rendering of White LED Light Sources (CIE 2007).

LED lighting requires completely different metrics for its assessment compared to the conventional light sources. Lumen output is a standard specification for a light fixture. Only total fixture lumens can serve as basis for valid comparisons between LED and conventional fixtures. For measuring conventional light sources and luminaires relative photometry is used, where the lumen output of a fixture's lamp is a reference, and the lumen output of the luminaire is measured relative to it. LEDs are typically inseparable from the luminaires, so absolute photometry has to be used. Only fixture lumens are measured. Therefore, fixture efficiency, which compares lamp lumens to fixture lumens, has no meaning for a LED luminaire (or in other words the efficiency is $100 \%$ ).

Luminous flux is not the best measurement of a LED luminaire, which can underestimate fixture's performance. Delivered light is the most relevant evaluation 
of the LED luminaire's performance. It describes how much useful light a luminaire can deliver to a task area. Useful light is the amount of fixture's light output that is effectively directed to a task area, discounting any wasted light (Weinert 2010).

A single LED has special features. It is a very bright light source and it is extremely small. Glare is an issue when LED lighting is implemented. The assessment of glare implies knowing the luminance. "Luminance is a measure of the light emitting power of a surface, in a particular direction, per unit apparent area" (DiLaura 2011 et al., p. 5.14)). It is the most important quantity in lighting design and illuminating engineering. Luminance is one of the direct stimuli to vision, and many measures of performance and perception (luminance of the source and background in glare analysis, luminance contrast for characterizing the task, etc.) (DiLaura et al. 2011).

As an example, LM-79-08 (IES) describes the procedures for performing standardized measurements of power, light output, and color characteristics of the LED technology (methods use sphere-spectroradiometer, sphere-photometer and goniophotometer). But none of the standards mentioned above provides procedure for obtaining luminances of LEDs. Division 2 in CIE (Physical Measurement of Light and Radiation) prepares the technical report (TC2-58) on measuring LED radiance and luminance (according to CIE website as of February 2012), but progress has not yet been published. Manufacturers do not provide luminance data on their products, since no standard developed for LED luminance measurements exists.

In a lighting environment luminance of an LED product must be measured. It is hard to find measured luminance of an LED. However, one conference paper reports $9.83 * 10^{6} \mathrm{~cd} / \mathrm{m}^{2}$ for high power white LED (Kohtaro et al. 2009). A good reference to compare this value to would be the luminance of the Sun. Prior to 
atmospheric attenuation the solar disk has a luminance of $1.6^{*} 10^{9} \mathrm{~cd} / \mathrm{m}^{2}$. The sun is roughly one-half of degree in diameter. If viewed directly sun can cause permanent physical damage to the eye due to sun's output in non-visible portion of the spectrum and extreme luminance (DiLaura et al. 2011).

For the purpose of acquiring luminance of an LED different approaches have to be considered. There are four ways to find out the luminance of a point in a certain direction:

1. To measure it with a conventional luminance meter;

2. To derive through the illuminance measurement and subsequent calculations;

3. Digital imaging photometer measurement;

4. And the non-traditional approach - High Dynamic Range Imaging technique.

Point by point measurements of light levels with a luminance meter like Minolta LS110 is a coarse and exhausting procedure. Performing lighting audits of premises requires a lot of effort and time. Moreover, these data are hard to analyze.

The calculation of luminance through illuminance measurements and reflective properties of the material can't always be done accurately. Simple formula stands true only for diffuse surfaces. And even more one needs to know the materials' coefficients of reflection. Direct illuminance measurements of a light source and subsequent calculations require a lot of time and effort as well.

The other way to measure luminance is obtain it with digital imaging photometers. They are extremely expensive, cumbersome and require some experience to work with (according to Radiant Imaging website as of October 2011). 
HDRI technique introduces the non-traditional approach of obtaining luminances in a lighting environment. It can store the information of a scene with the range of many orders of magnitude, and values can be photometrically correct.

Scene dynamic range is defined as (Xiao et al. 2002):

$$
\begin{gathered}
\mathrm{DR}_{\text {scene }}=\frac{Y_{\max }}{Y_{\min }} \quad(2.1), \text { where } \\
\mathrm{Y}_{\max }-\text { maximum scene luminance, } \\
\mathrm{Y}_{\min }-\text { minimum scene luminance. }
\end{gathered}
$$

For natural scenes it is typical to have the range of 10000 for luminance distribution. As an example, window with the view of sky can have the order of $10000 \mathrm{~cd} / \mathrm{m}^{2}$, while the floor under the table might be as low as $1 \mathrm{~cd} / \mathrm{m}^{2}$ (Xiao et al. 2002).

Term "dynamic range" for the image is the ratio between the lightest and darkest pixel which are considered to be outliers. The most robust definition will exclude certain percentage of the lightest and darkest pixels (Reinhard et al. 2010). It is important to understand that dynamic range of the image is affected by three main components in the imaging pipeline: the optics, the sensor and the color transformations (Xiao et al. 2002).

HDR image can be created by taking multiple photographs of the same scene at different exposure levels and merging them to generate the original dynamic range of the captured scene (Reinhard et al. 2010, Banterle et al. 2009). The new HDRI tool for capturing lighting scene at a high resolution within a large field of view in a quick and inexpensive manner can provide researcher with lots of opportunities and flexibility. It allows getting thorough information about existing lighting conditions. HDRI can be applied in lots of scientific applications such as luminance display (falsecolor and isocontour), luminaire performance testing, glare analysis, luminance 
derivation, imaged-based lighting rendering, human vision simulation and some others (Guglielmetti et al. 2011).

The human visual system (HVS) can adapt to luminances in a very wide range $\left(10^{-6}-10^{6} \mathrm{~cd} / \mathrm{m}^{2}\right)$, while a single photograph can capture only a limited range. At any given eye adaptation humans can see details in regions that vary $1: 10^{4}$. In order to interpret visual environment eye and brain work together, both are parts of visual image processing system. Optical system of the eye forms the image of the observed scene onto the retina. Photoreceptors absorb the light, and convert it to electrical signals. Optical nerve transmits electrical signals to the area of the brain called visual cortex that processes these signals. And then the perceived image is produced. The HVS is responsible for our vision.

There are two types of photoreceptors in retina: cones and rods. They are responsible for photopic and scotopic vision respectively. The fovea, the central area in the receptive field, contains cones responsible for the daylight vision (sensitive to $10^{-2}-10^{8} \mathrm{~cd} / \mathrm{m}^{2}$ ) numbering around 6 million. Three types of cones are responsible for our color vision. In photopic vision fine details are resolved in the fovea and color is perceived. The rods numbering around 90 million are responsible for the nighttime vision. They are sensitive to luminances in the range of $10^{-6}-10^{-2} \mathrm{~cd} / \mathrm{m}^{2}$. Fovea doesn't have any rods. In the scotopic vision there's no perception of color. Resolution of the detail occurs in the periphery. Mesopic range is the range when both types of photoreceptors are activated $\left(10^{-2}-10 \mathrm{~cd} / \mathrm{m}^{2}\right)$. It's intermediate between the photopic and scotopic states (Reinhard et al. 2010).

Our eyes have the ability to dynamically adjust to a given scene, while camera captures a single still image (according to Cambridge in Color website as of September 2011). In addition, computer display technology doesn't deliver images in 
the range of luminances that HVS is capable to capture. Display range has about two orders of magnitude between minimum and maximum luminance. Maximum display luminance for the well-designed cathode ray tube (CRT) is around $100 \mathrm{~cd} / \mathrm{m}^{2}$, and for LCD display it's about $300-400 \mathrm{~cd} / \mathrm{m}^{2}$. These levels do not even start to approach daylight levels. Traditional computer image files do not store information in photometric units $\mathrm{cd} / \mathrm{m}^{2}$ (Reinhard et al. 2010). Traditional imaging methods (consumer-grade cameras) are usually constrained by limitations in technology -8 bits per color channel or pixel. It is called low dynamic range (LDR). Therefore, much of the luminance information available in a scene is lost (Moeck et al. 2006).

HDRI has revolutionized the field of computer graphics, photography, visual reality, visual effects, and video game industry. Now real-world lighting can be captured, stored, transmitted and used in various applications. Both very dark and very bright areas can be stored at the same time. Information in HDRI allows applying advanced tone mapping or calibrated false color luminance images. To display an HDRI image on conventional screen tone mapping is used. It is the operation that changes the dynamic range of the HDR content to fit the lower dynamic range available on the given display (Reinhard et al. 2010, Jacobs 2007).

The initial purpose of the HDRI technique was not intended towards measuring luminance. Therefore, it has to be evaluated for this type of application.

HDRI technique is already widely used in lighting research. There are studies on assessing HDRI's accuracy for various lighting applications (Inanici 2006; Moeck et al. 2006; Moeck 2007; Inanici 2010; Konis et al. 2011; Cai et al. 2011). The HDRI tool still has to be evaluated for accuracy and reliability in the lighting field to gain more statistical data and to expand HDRI's abilities. 
This research investigates the ability of the HDRI technique to accurately capture luminance values of a bright light source (a single LED chip) with a narrow light distribution.

\subsection{Theory of High Dynamic Range Imaging with the application in Lighting}

\subsubsection{Outline of making an HDRI}

The following sections cover the process of making an HDRI and provide practical guidelines for the HDRI acquisition.

\subsubsection{Process of making an HDRI}

In order to obtain High Dynamic Range Image a sequence of photographs of a scene with various exposure times should be taken with specific settings (see section 2.2.1.2). Exposure is a key concept. It is determined by one of the three settings in a digital camera: aperture size (determines the ray cone angle; how much light can enter a camera); shutter speed (controls the exposure duration); and ISO-speed (controls the sensitivity) (according to Cambridge in Color website as of September 2011). Control of one of the three settings allows changing the exposure.

Response curve (RC) of a camera is a non-linear relationship between image's irradiance and scene's radiance (Mitsunaga et al. 1999).Usually manufacturers do not provide camera's response function. Camera makers apply various algorithms to modify RC to enhance the contrast, saturation, and other parameters of the photographs. In order to provide softer highlights and reduce noise visibility in the shadows ends of the curves are modified as well. But as long as the input is not altered by the camera from one exposure to the other, it is possible to recover camera's response function from an appropriate sequence of images. So, the first step in making an HDRI is to obtain camera response function, which is done only once for a specific camera and lens combination (see section 2.2.2), then it can be re-used. 
While taking photographs with various exposures, each image in a sequence will contain pixels that are underexposed, properly exposed, and overexposed. But the most important point is that every pixel will be properly exposed in one or more images in a sequence. If we assume that our device is linear then in order to bring every exposure into one domain, each pixel has to be divided by the image's exposure time. As soon as each image is in the same units of measurements, corresponding pixels may be averaged across exposures. Under- and overexposed pixels should be excluded (Reinhard et al. 2010). There are some examples of response curves from different manufacturers on figures 2-1 and 2-2. They illustrate that a high-order polynomial may be used to model the response function.

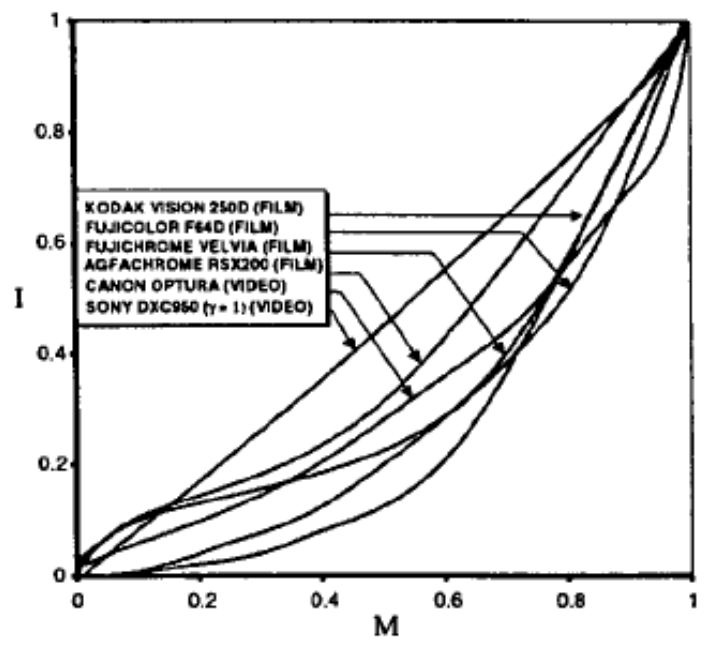

Figure 2-1. Response functions of a few popular films and video cameras provided by their manufacturers (Mitsunaga et al. 1999)

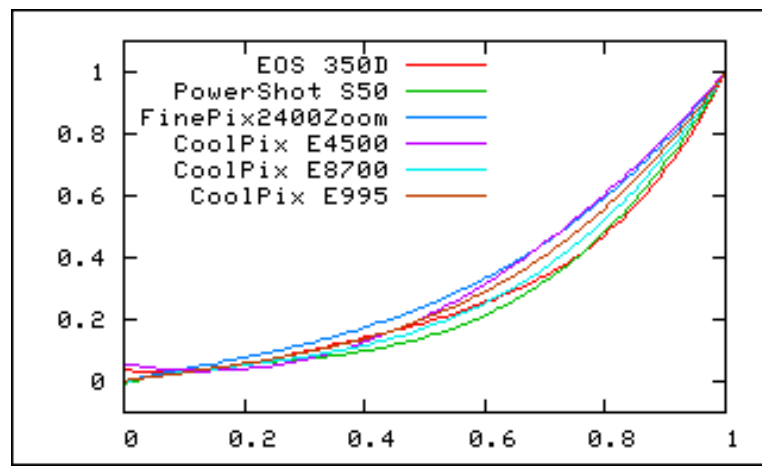

Figure 2-2. Response functions of a few popular cameras (according webHDR website as of September 2011) 
In order for HDR software to merge LDR images, it has to know exposure values (Jacobs 2007):

$$
E V=\log _{2}\left(\frac{f^{2}}{T} \frac{S}{100}\right) \quad \text { (2.2), where }
$$

$\mathrm{f}$ - aperture,

$\mathrm{T}-$ shutter speed,

$\mathrm{S}-$ ISO setting.

Standard JPEG file has all this information in exchangeable image file format EXIF header of the image (according to EXIF website as of October 2011). All parameters of the image and all the settings are stored in this standard.

One should be aware of some errors that are introduced in the exposure value of an individual LDR image. The error in aperture number is the first one, which is caused by limited repeatability of diaphragm inside the lens. The second one is the exposure time. Shutter speed is rounded on the screen (eg. 1/30, 1/60, while it is $1 / 32$, 1/64). So, these errors add up, and additional calibration would provide more accurate luminance readings per pixel of an HDRI (Jacobs 2007).

After obtaining response curve the next step is to make an absolute photometric calibration. This can be done by taking a reading on a grey card or uniform area in the scene with the luminance meter.

In glare evaluation (physiological evaluation of the luminous environment) additional spatial or photogrammetrical calibration is required. Besides knowing the object's luminance it's necessary to know the position of a pixel with regards to the camera and the solid angle subtended (Jacobs 2007).

The next step is to fuse low dynamic range images of multiple exposures with the obtained response curve to create an HDR image of the analyzed scene with the 
software. Steps of making an HDRI are shown on figure 2-3. Obtained image can be used for various purposes like analysis of the lighting environment (falsecolor technique giving luminance values within the scene) (Reinhard et al. 2010).

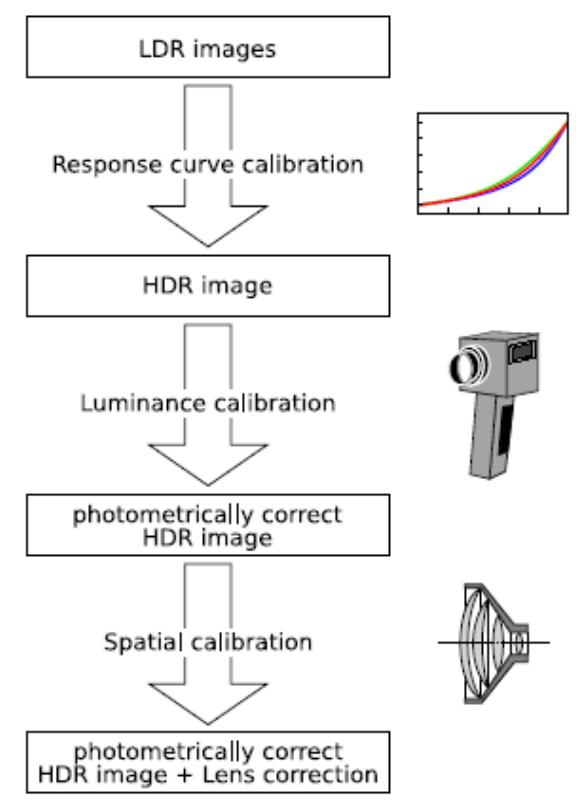

Figure 2-3. HDRI acquisition process (Jacobs 2007)

\subsubsection{Practical guidelines of making an HDRI}

In order to make a successful HDRI, it's necessary to follow some practical guidelines.

First, it is necessary to obtain camera's responsive curve. Usually digital number $\mathrm{Z}$ is a nonlinear function of the original exposure $\mathrm{X}$ at the pixel (Mitsunaga et al. 1999). Then along with the photometric calibration with a luminance meter digital camera can be used as a luminance mapping tool.

While capturing a sequence of images, it's necessary to use aperture priority mode of a camera (user sets aperture size and ISO-speed, camera sets shutter speed) or manual exposure mode (all settings are set by the user). In order to create a successful HDRI low dynamic range images have to be identical. Changing aperture size introduces problems with the optical vignetting (light fall-off towards the edges). 
The depth of field is changed as well. When ISO setting is set to a higher value more noise is introduced. The best option to create an HDRI is to change the exposure time;

Camera's white balance has to be fixed on daylight (D65); ISO 100;

All settings in the camera such as color and contrast enhancement have to be turned off. It would be less likely for a camera to make color transformations; Tripod is necessary, so there would be no movement between taking images, therefore less alignment would be needed later. It's more convenient to obtain a sequence automatically, for example using the autobracketing feature in a camera or to control it through the computer. Thereby less influence of camera and objects' movement will occur in the sequence;

A scene for obtaining response curve should have large grey or white surfaces with very bright and very dark areas. Those will provide nicely continuous gradients for the software to sample. The closer the scene to a neutral grey (noncolored), the better;

A sequence of exposure bracketed images should be separated by 1 exposure value (EV). This is equivalent to halving or doubling the exposure. The number of bracketed exposures depends on a model of a camera. The more exposures camera has the higher dynamic range can be captured. Most scenes can be effectively captured with nine exposures, or even with 5-7 (Reinhard et al. 2010). The minimum number of photographs needed to recover a radiance map given camera's response curve is a function of radiance range in a real scene. $\mathrm{R}$ - the whole range of radiance values of the scene, $\mathrm{F}$ - camera's working range. So, the minimum number is $\mathrm{R} / \mathrm{F}$ (Cai et al. 2011). 
- $\quad$ Photos histograms allow checking if the scene was properly captured. Histogram of the photo is a chart that shows the number of tones being captured at each brightness level (Busch 2011). Some cameras have two types of histograms: one is for the overall brightness of an image, and alternate version with separate red, green, and blue channels of the image. Horizontal axis shows the brightness level starting from the left 0 , which is black, to the right 255 , which is white. Each vertical line out of 256 shows the number of pixels in the image at each brightness level. If there are no pixels at the particular brightness level, that would be shown by no bar at a particular position. The more pixels of certain brightness are in the image the taller the bar. For example, if the image was badly underexposed, the shape of the histogram would be shifted to the left - to darker tones. The opposite situation occurs for the overexposed image;

"If, however, on the darkest image (shortest exposure), some or all parts of the bright sources are either over-exposed (they have a value of 255 in the JPEG), or even if they are above 200, then the HDR engine can't reliably work out the true luminance and will simply cut it off." The recommended range is above 27 for the longest exposure and below 228 for the shortest in 8-bit domain (HDRI mailing list as of February 2012);

Luminance of a uniformly lit grey card in the scene has to be measured with a luminance meter for the absolute calibration;

- $\quad$ Determine the luminance in the HDR image;

- Compute the calibration factor, which is simply the ratio of two:

$$
\mathrm{CF}=\text { Luminance }_{\text {Real }} / \text { Luminance }_{\mathrm{HDR}}
$$

This factor will be around 1.0 (according to webHDR website as of September 2011, Reinhard et al. 2010). 


\subsubsection{Response curve of a specific camera and lens combination}

Non-linear relationship (or mapping) between image's irradiance and scene's radiance is called radiometric response function of the imaging system (Mitsunaga et al. 1999). Some algorithms developed by various authors derive an approximate response function without radiometric calibration, which implies measuring RGB response of the camera in the range of 380-780 nm with the monochromator.

The relationship of the image irradiance $\mathrm{E}$ to scene radiance $\mathrm{L}$ is determined in the sequence provided below ("Introduction to Computer Vision" as of October 2011).

Due to physical property of imaging systems known as reciprocity (figure 2-4), we have:

$$
d \omega_{i}=d \omega_{s}
$$

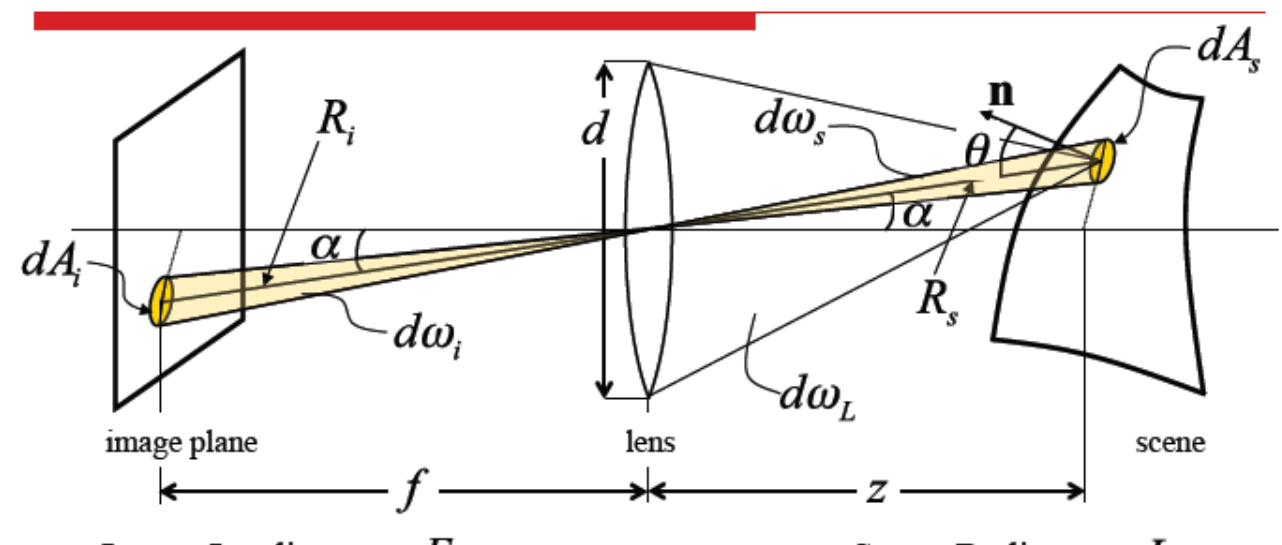

Image Irradiance: $E$

Scene Radiance: $L$

Figure 2-4. Relationship between image irradiance $E$ and scene radiance L ("Introduction to Computer Vision" as of October 2011)

Then, according to the definition of the solid angle:

$\frac{d A_{i} \cos \propto}{R_{i}^{2}}=\frac{d A_{s} \cos \theta}{R_{S}^{2}}$

From the geometry: 
$\frac{d A_{i} \cos \alpha}{(f / \cos \alpha)^{2}}=\frac{d A_{s} \cos \theta}{(z / \cos \alpha)^{2}}$

Recombination:

$\frac{d A_{s}}{d A_{i}}=\frac{\operatorname{co} \propto}{\cos \theta}\left(\frac{Z}{f}\right)^{2}$

Solid angle subtended by the lens:

$$
d \omega_{L}=\frac{\pi d^{2}}{4} \frac{\cos \alpha}{(z / \cos \alpha)^{2}}
$$

Then, flux received from projected area of the scene equals to the flux projected to the image:

$$
d \varphi=L\left(d A_{s} \cos \theta\right) d \omega_{L}
$$

And image irradiance equals to:

$$
E=\left(\frac{d \varphi}{d A_{i}}\right)
$$

To obtain the dependence of image irradiance on scene radiance, following steps are performed. Substitute flux (2.9) into the image irradiance definition (2.10); then substitute $d A_{S} / d A_{i}(2.7)$ and $d \omega_{L}(2.8)$. After reducing the fraction you get the relationship of the image irradiance $\mathrm{E}$ and the scene radiance L:

$$
E=L \frac{\pi}{4}\left(\frac{d}{f}\right)^{2} \cos ^{4} \alpha \quad(2.11), \text { where }
$$

$\mathrm{d}$ - diameter of the aperture;

$\mathrm{f}$ - focal length of the imaging lens;

$\alpha$ - angle subtended by the principal ray from the optical axis.

Thus, image irradiance is proportional to the scene radiance (Ko Nishino 2010).

For the ideal imaging system the image brightness would be (Mitsunaga 1999):
$I=E t$
(2.12), where 
$\mathrm{t}$ - the time that image detector is exposed to the scene.

For the ideal imaging system the radiometric response would be linear:

$$
\begin{aligned}
& I=L k e \\
& k=\cos ^{4} \varphi / h^{2} \\
& e=\left(\pi d^{2} / 4\right) t
\end{aligned}
$$

- $\quad$ is exposure of the image, which can be changed either by altering aperture diameter $(\mathrm{d})$ or the duration of exposure $(\mathrm{t})$.

But unfortunately the relationship between image's irradiance and scene's radiance is not linear due to various non-linearities like "gamma" correction, image digitizer, inclusive of A/D conversion, etc. (Mitsunaga et al. 1999). And also relationship is non-linear due to manufacturers' applications of some tone mapping operators that make an image look better (Moeck et al. 2006). So, radiometric response function has to be derived.

In (Debevec et al. 1997) the goal of the research was to recover camera's response curve by having only a set of photographs of various exposures, and further using pixel values of the image to reconstruct radiance picture of the captured scene. The idea of the approach was to obtain information about pixel location at various exposures (figure 2-5). The speed setting of the camera provides user with the relative exposure ratios. Thus, the shape of the camera's response curve is known at those three parts. The next step is to find a way to fit curve parts together (Moeck et al. 

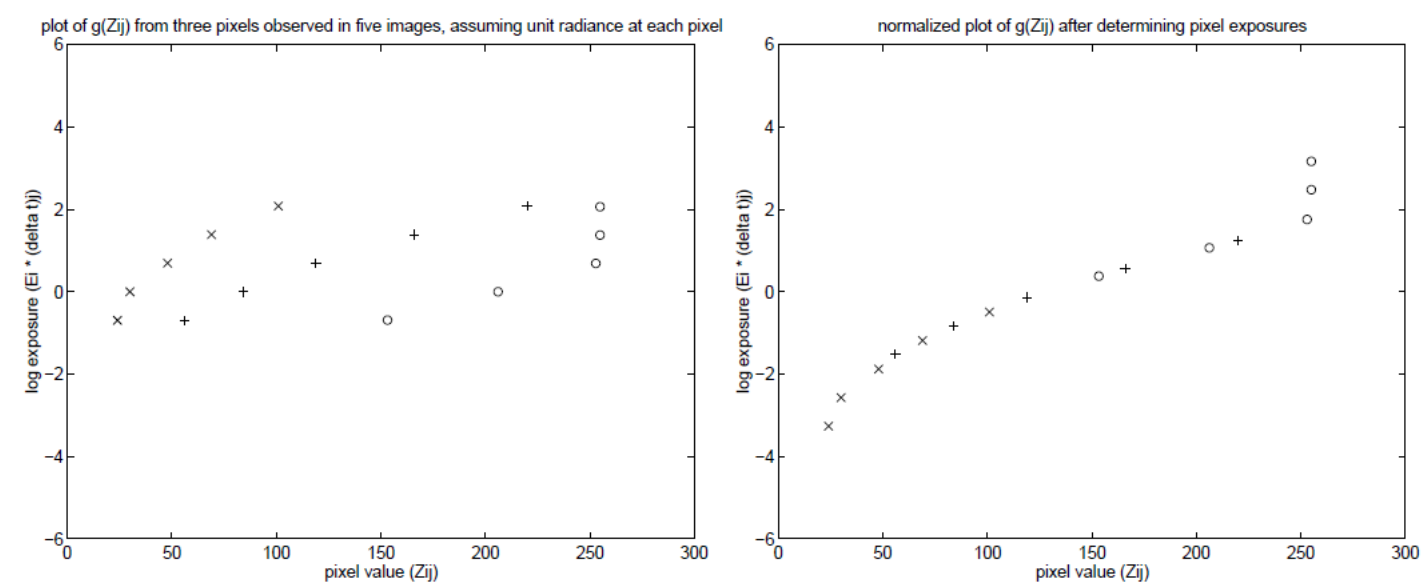

Figure 2-5. Recovering camera's response curve. $X$ - represents part of the $\mathrm{g}$ curve obtained from the digital values at one pixel for five different known exposures (equation 2.16). Position of the curve is arbitrary corresponding to the unknown $\ln E_{\mathrm{i}}$. Symbols + and ${ }^{\circ}$ represent two other pixels. The idea is to "line up" them into a single smooth, monotonic curve (Debevec et al. 1997)

Debevec and Malik used linear optimization to find a smooth curve with a minimized mean-square error.

After deriving camera's response curve assuming that the exposure is known, the function can be used to convert pixel values to relative radiance values (Debevec et al. 1997).

$$
\ln E_{i}=g\left(Z_{i j}\right)-\ln \Delta t_{j} \quad(2.16), \text { where }
$$

$\mathrm{Z}_{\mathrm{ij}}$ - pixel value ( $\mathrm{i}$ - spatial index over pixels, $\mathrm{j}$ - over exposure),

$\Delta \mathrm{t}_{\mathrm{j}}-$ exposure times,

$\mathrm{E}_{\mathrm{i}}-$ irradiance values,

$\mathrm{g}=\mathrm{f}^{-1}$, where $\mathrm{f}$ is tone mapping processing.

So, in order to capture the whole high dynamic range all available exposures for a particular pixel should be used to compute its radiance (equation 2.17). Also, the weighting function is implemented to give higher weight to exposures in which pixel's value is closer to the middle of the response curve (equation 2.18). 


$$
\begin{gathered}
\ln E_{i}=\frac{\sum_{j=1}^{P} w\left(Z_{i j}\right)\left(g\left(Z_{i j}\right)-\ln \Delta t_{j}\right)}{\sum_{j=1}^{P} w\left(Z_{i j}\right)} \\
w(z)= \begin{cases}z-Z_{\min } & \text { for } z \leq \frac{1}{2}\left(Z_{\min }+Z_{\max }\right) \\
Z_{\max }-z & \text { for } z>\frac{1}{2}\left(Z_{\min }+Z_{\max }\right)\end{cases}
\end{gathered}
$$

Response curve $\mathrm{g}(\mathrm{z})$ will typically have a steep slope near maximum and minimum pixel values $\left(Z_{\max }\right.$ and $\left.Z_{\min }\right)$, so there's an expectation that it will be less smooth. This means the curve will fit the data more poorly near extremes. It explains the reason for giving higher weight to exposures in the middle of the curve. This algorithm by Debevec works pretty well with images that are not too noisy.

The other algorithm is introduced in (Mitsunaga et al. 1999). Their approach in deriving a polynomial approximation of the response function doesn't require precise exposure inputs. Authors resolve the exact exposure ratios in addition to the camera response function.

In (Robertson et al. 2003) authors present new iterative procedure of recovering camera's response curve. Also, new weighting function for the input images for an HDRI reconstruction is implemented. Higher exposure times are weighted more heavily. The technique is based on a probabilistic formulation.

One more way of recovering camera's response function is presented in (Herwig et al. 2009). The focus of the paper is to recover the response curve with non-iterative methods with a minimum set of input values.

Camera's response function in combination with the exposure information of the LDR images should provide radiometrically correct pixel information.

\subsubsection{Software and calculations discussion}

Currently, there's a great number of software available for the HDRI creation. Among software presented on the market are: Photosphere, Picturehaut, WebHDR, 
hdrgen, bracket, HDR Shop, Luminance HDR, Hugin, Photomatix, CinePaint, and Photoshop (Guglielmetti et al. 2011). Photosphere is one of the most widely used.

\subsubsection{Color space}

Most cameras have their own native color space because of the various spectral responsitivities of the sensors. Converting between various device-dependent color spaces is a straight forward procedure, but it's more convenient to have a single standard color space. Unfortunately, there are a couple of standards. One version of image encoding is "output-referred standards". This standard uses a color space that corresponds to a specific output device, so no additional resources are wasted on colors that are out of the device gamut. But the disadvantage of such encoding is that colors that can be represented on a specific device can't be represented on others. The other version of the standard is "scene referred". The goal is to portray original captured scene as close as possible. But then the requirement of applying some tone mapping to the pixels to fit device's gamut occurs. Tone mapping can be represented by simple clamping values in a range of $[0,1]$ or applying compression based on the studies of human visual system and its operation. The major advantage of such a standard is that correct output can be produced on any device.

sRGB color space is specified by the International Electrotechnical Commission, and many digital cameras produce images in this color space. Luminance of a particular pixel of an image can be computed from the linear combination of the RGB components. Therefore, one has to know the primaries of the camera-depended color space, and the white point (Moeck et al. 2006). Nonlinear sRGB color space is based on a virtual display. It has the following primaries and the white point in terms of chromaticities $(x, y)$ : $R(x=0.64, y=0.33), G(x=0.30, y=0.60)$, 
$\mathrm{B}(\mathrm{x}=0.15, \mathrm{y}=0.06)$, illuminant is $\mathrm{D} 65$, white point $(\mathrm{x}=0.3127, \mathrm{y}=0.3290)$. The maximum luminance for the white is $80 \mathrm{~cd} / \mathrm{m}^{2}$ (Reinhard et al. 2010).

\subsubsection{Luminance values in the software}

A radiometer that has been optically or electronically filtered to approximate a spectral sensitivity function of the fovea is called a photometer. Therefore, luminance can either be obtained through measurements with a radiometer and a physical photopic filter or through electronic derivation of luminance values.

Since a regular digital photo camera does not have a photopic filter to account for the human vision (CIE photopic luminous efficiency curve $V(\lambda)$ ), therefore a second way to obtain luminance is used in the HDRI technique.

If a pixel color is specified in a device-independent RGB color space, its luminance may be computed from a linear combination of the red, green, and blue components. Photometric data can be generated from HDR images through a series of calculations that involve conversion from RGB to CIE XYZ values for each pixel (Inanici 2006).

Photosphere derives luminance in the following sequence:

- $\quad$ Obtain CIE XYZ values for each pixel from sRGB standard camera color space (using CIE Standard Illuminant D65, and standard CIE Colorimetric Observer with 2 field of view) by converting one color space to the other;

- $\quad$ Then luminance can be computed since the Y component in XYZ color space represents luminance $\mathrm{L}(\mathrm{V}(\lambda)=\overline{\mathrm{y}}(\mathrm{x}))$. Thus, $\mathrm{L}$ is the linear combination of the red, green, and blue components.

$L=k *(0.2127 * R+0.7151 * G+0.0722 * B) \mathrm{cd} / \mathrm{m}^{2} \quad$ (2.19) , where 
- $\quad \mathrm{k}$ is a constant used to calibrate images with a physical luminance measurement of the selected region in the scene. Can be applied either to the pixel values in an image or to the camera response function.

The calculations in Photosphere are based on CIE chromaticities for the reference primaries (sRGB) and CIE Standard Illuminant D65:

$$
\begin{aligned}
& R(x, y, z)=(0.64,0.33,0.03) \\
& G(x, y, z)=(0.30,0.60,0.10) \\
& B(x, y, z)=(0.15,0.06,0.79) \\
& \text { D65 }(x, y, z)=(0.3127,0.3290,0.3583)
\end{aligned}
$$

Shooting with white balance mode set to D65 assures that measurement condition matches the sRGB color space. In Radiance calculations are based on equal energy white point $(\mathrm{x}, \mathrm{y}, \mathrm{z})=(0.330 .330 .33)$. Different computation is the reason for minor difference between original luminance calculations in Radiance $\left(\mathrm{R}^{*} 0.265+\right.$ $\left.\mathrm{G}^{*} 0.067+\mathrm{B}^{*} 0.065\right)$ and the calculations in Photosphere $\left(\mathrm{R}^{*} 0.2127+\mathrm{G}^{*} 0.7151+\right.$ B*0.0722) (HDRI mailing list as of September 2006).

\subsubsection{Optical vignetting}

Vignetting is a light fall-off towards the periphery of an image due to the blocking of some incident rays by the effective aperture size (Kim et al. 2008). The effect of optical vignetting becomes significant as the aperture size increases and vice versa. Some authors implement "digital filter" to reduce unwanted vignetting effect (Chung et al. 2010, Anaokar et al. 2005, Moeck et al. 2006, Inanici 2006).

Zoom lens has a special featured compared to prime one. In a zoom lens the aperture (d) is changed with the aperture setting as well as with the focal length (f). Even at the same f-stop (relative size of aperture, $\mathrm{N}=\mathrm{f} / \mathrm{d}$ ) the vignetting effect can be different, since one aperture stop can be obtained with many different combinations of 
aperture sizes and focal lengths. Thereby, if the aperture is set to a specific number, when zooming in/out the vignetting effect will be different.

Longer focal lengths (narrow angle of view) decrease the quality of the HDR images of light-emitting surfaces. The trend can be improved at smaller apertures (Cai et al. 2011). According to author the reason for lower quality might be the increased light scattering and lens flare when camera is zoomed in at a light source. This can be alleviated by increasing the ambient light level for increased signal-to-noise ratio of image sensors with low light sensitivity. Higher ambient light levels significantly improve quality of the HDR images of light-emitting surfaces. Average percentage error for light-emitting surfaces is $6.6 \%$.

\subsubsection{Other issues}

Other important issues to consider are noise, point spread function and distortion.

Noise in the image occurs with high ISO-setting and long exposures (when random photons are registered). Dark frame subtraction is recommended for exposures longer than one second. Photo of blank exposure with random pixels is compared with the original photo. Noise is represented in the pixels with the same characteristics in both images (Busch 2011). It is important to keep in mind that some information can be accidentally lost with the noise.

Factors such as aperture size, exposure time, distance from the optical center all affect the point spread function (PSF). This optical effect occurs when narrow light beam spreads out and scatters by the lens. It is an inherent property of the optical systems. The result is a reduction of pixel's luminance due to the falling off of some amount of light on surrounding pixels (Chung et al. 2010, Reinhard et al. 2010, Rea et al. 1990, Moeck et al. 2006, Inanici 2006, Anaokar et al. 2005, Jacobs 2007). 
Resolution of an image can be characterized by the PSF or the Modulation Transfer Function (MTF).

The PSF may be determined computationally by summing up all hot pixels, that is, pixels above a certain threshold, in a separate grey scale image (Reinhard et al. 2010). Then it is a straightforward procedure to remove the PSF from the HDR image by simply subtracting the PSF multiplied by the value of the hot pixel from its neighbors.

In (Xiao et al. 2002) authors investigate how diffraction and lens flare influence the dynamic range. The components of the flare are illustrated on figure 2-6. Flare consists of (a) star-like PSF, (b) triangular ghost image, (c) haze.

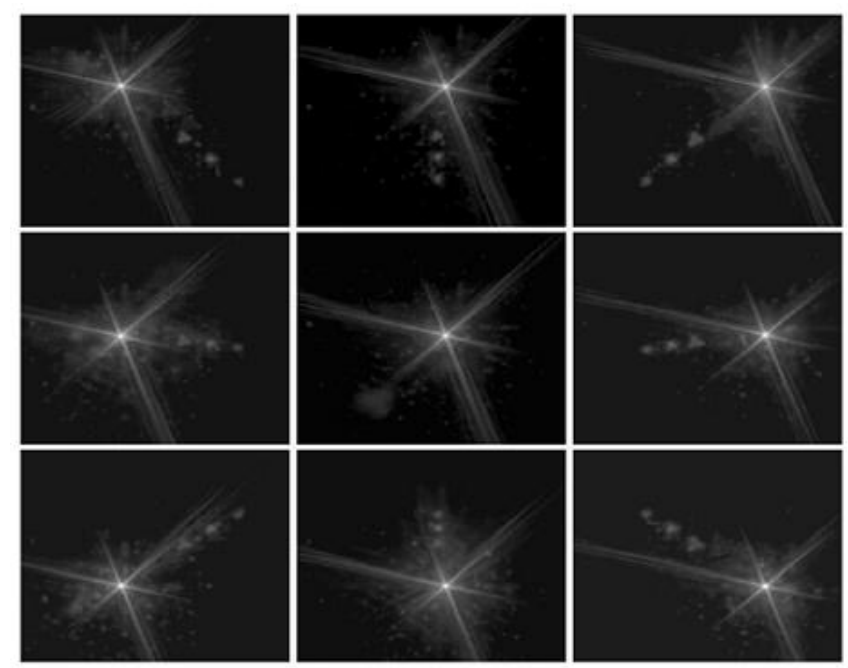

Figure 2-6. Images of flare components of a point light source at different locations (Xiao et al. 2002)

Star-like component (a) is caused by the diffraction. The amplitude drops to $0.1 \%$ of peak value at 1.5 degrees away from the star's center. Ghost images (b) are caused by reflections from different lens surfaces from the group of lens elements. The amplitude of the ghost image at 20 degree away from its peak is still $0.01 \%$ of the peak value. The veiling glare (c) is spread throughout the image. As aperture size increases, the amplitudes of these flare components slowly decrease. The limit on the 
effective dynamic range of the capture system is set by the optical components. Thus, dynamic range is scene-dependent.

Distortion plays a considerable role with the bigger field of view of the camera. The distortion needs to be checked at the minimum zoom (Moeck et al. 2006).

\section{3 Studies of HDRI technique validation for lighting measurements}

HDRI is a valuable tool in lighting research. It offers a variety of options for lighting analysis such as: luminance evaluation, illuminance derivation, glare analysis, image-based lighting, luminaire performance testing, etc.

In order to capture the wide luminance range of the scene with the HDRI photography, multiple exposure photographs are taken. Self-calibration process from a sequence of photographs allows the computational derivation of the camera response function. So, if a digital camera has a multiple exposure capability, then it can be used for the HDRI. Then with the known RC of the camera photograph sequence is combined into a single HDR image. HDRI photography was not specifically developed for lighting measurements. So, some studies evaluating HDRI technique applied to measurements of lighting environment were done by some authors (see table A in Appendix A).

\subsubsection{Studies of the HDRI technique validation in a scene with no bright light} sources

In "Evaluation of high dynamic range photography as a luminance data acquisition system" multiple exposure photographs were taken with a digital camera Nikon Coolpix 5400, and processed using software "Photosphere". Then reference physical measurements were taken with a calibrated Minolta LS110 luminance meter with $1 / 3^{\circ}$ field of view. For the determination of the camera's response function an 
interior scene with daylight with large and smooth gradients was chosen. The goal of the study was to compare physically measured luminance of a particular part in the scene with the one derived from the HDR image. Measurements were taken under various conditions: indoors and outdoors, and with various lighting sources.

Among measured targets were: 24 squares with reflectances in the range of 4$87 \%$, grey square target with $28 \%$ reflectance against white-then-black surrounding, and then black-then-white background. And among used targets was a Macbeth ColorChecker. Among light sources used in the research were an incandescent lamp, a projector (using 500W tungsten lamp), fluorescent T12, T8, and T5 lamps with CCT of $6500 \mathrm{~K}, 3500 \mathrm{~K}$, and $3000 \mathrm{~K}$ respectively, metal halide, and high pressure sodium lamps. Depending on the spectral power distribution of a light source the error margins for greyscale and colored targets varied.

The total number of targets of 485 under various light sources showed the following results. The average percentage error for all targets was $7.3 \%$, for greyscale and colored $5.8 \%$ and $9.3 \%$ respectively. Minimum target luminance was $0.5 \mathrm{~cd} / \mathrm{m}^{2}$, while the maximum was $12870 \mathrm{~cd} / \mathrm{m}^{2}$.

Increased error was observed for the darker greyscale targets. Luminances of the darker regions are over-estimated due to general light scattering in the lens. And the luminances of the saturated color samples show the increased error.

The research by Inanici shows that the HDRI technique gives reliable results capturing wide range of luminances with an accuracy of $10 \%$. In order to have absolute validity this method requires calibration with the luminance meter (Inanici 2006).

In (Anaokar et al. 2005) authors tested the accuracy of high resolution luminance map generated by the Photosphere software. Besides comparing the 
accuracy of three different cameras (Nikon Coolpix 5400 was chosen for the study), errors were estimated due to the usage of various colors of Munsell chips, spectral power distribution of a light source, optical vignetting of the camera, and spatial resolution of the object.

Authors calculated the error depending on color. Six Munsell cards of different colors were used, each consists of 16 chips as shown on figure 2-7.

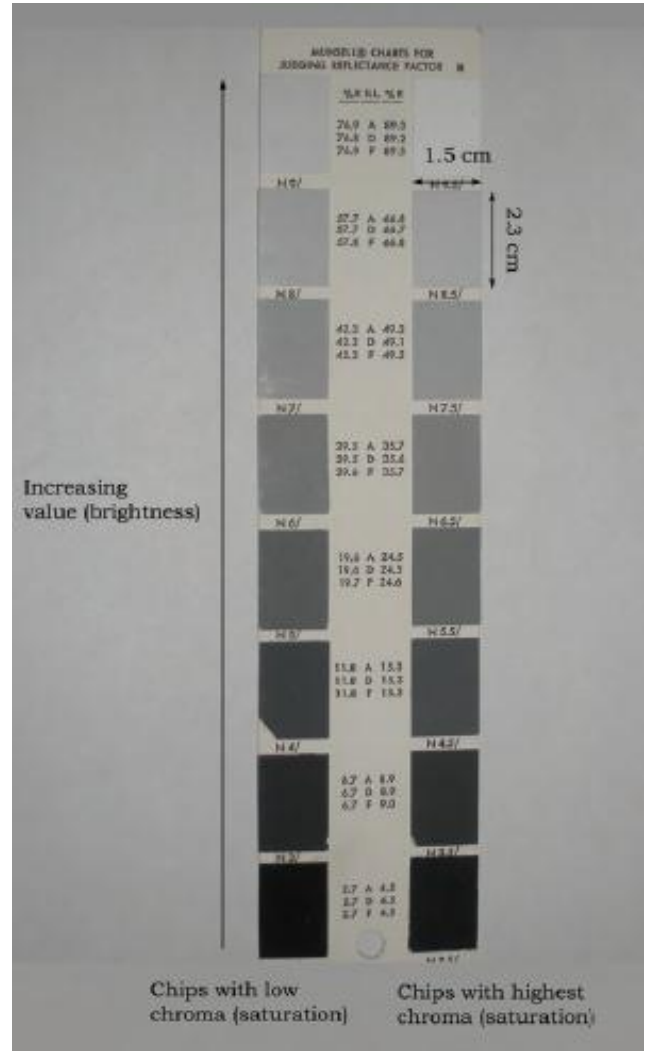

A

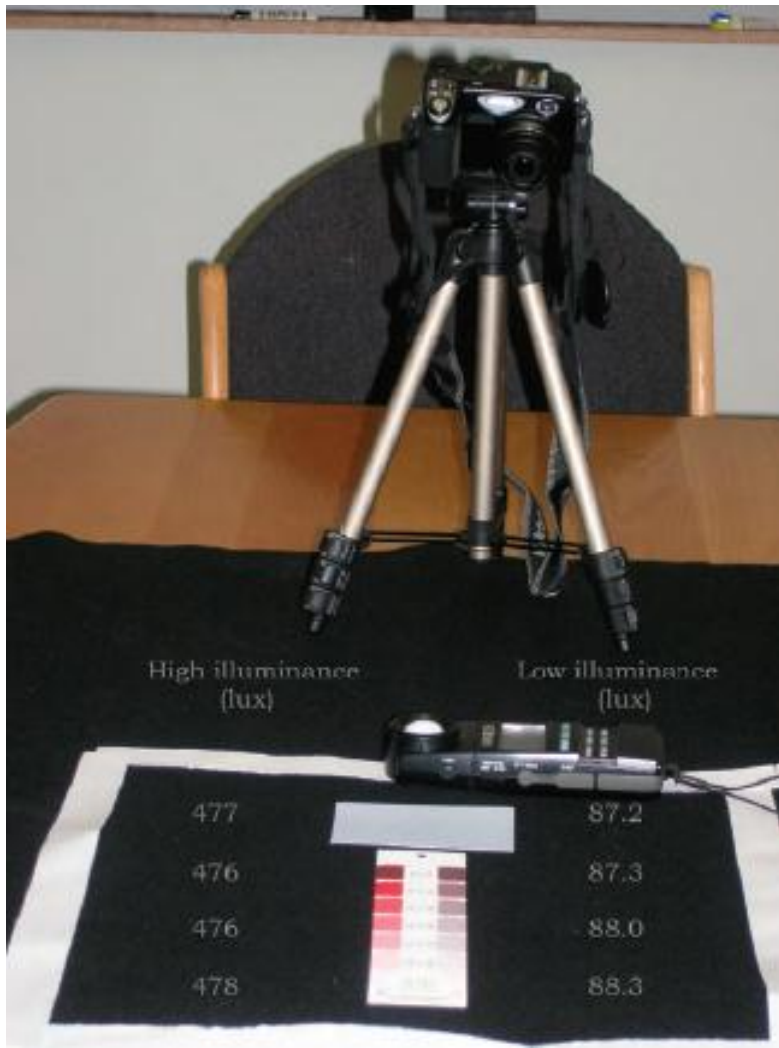

B

Figure 2-7. A- Munsell chip, B - Experiment set-up (Anaokar et al. 2005)

Reflectance of each chip of a Munsell card was specified for a specific light source. Twelve images were obtained varying shutter speed in one step increments, which is more reliable than changing aperture size (Debevec et al. 1997, Mitsunaga et al. 1999). HDRIs were obtained in the Photosphere software. Then illuminance 
measurements were taken for the grey card of $30 \%$ reflectance. Then luminance was computed as:

$$
L=\frac{E \rho}{\pi}
$$

This luminance was used to calibrate the image. Generated images gave authors luminances that were used to compute the reflectance for each sample. Then obtained values were compared to the actual coefficients of reflectance.

Results of the experiment revealed the following. Cool hues have the largest errors compared to warm hues. Spectral responsivity calibration is suggested to overcome the problem of large errors for surfaces with reflectances less than $20 \%$ (Jacobs 2007). The error in reflectance increases with saturation and with the decrease of Munsell value. Images fused in Photosphere show higher luminances for colors with a low value, or darker colors of the same hue and chroma. Errors in reflectance were similar for light sources with various spectral power distributions. For other results see the paper (Anaokar et al. 2005).

In (Moeck 2007) author investigates the accuracy of measurements done with the camera with CMOS (complementary metal oxide semiconductor) sensor (Canon EOS 350 D Digital Rebel XT, a digital SLR camera with 8 megapixels). Author overcomes the disadvantages of fisheye lens (cost, vignetting problems, lens flare, and only hemispherical field of view) by using mirror spheres. Due to dynamic outdoor lighting conditions it is important to capture panoramic maps that offer a full field of view around one axis. The paper presents results on verifying luminances of grey scales and matte color checkers obtained from HDR images. Luminance errors of spherical HDR images obtained with a mirror ball were calculated.

The results reveal that HDR luminances of most hues can be reasonably estimated. Light surfaces are underestimated, and dark surfaces are overestimated. 
Measurements of dark colors with high chroma result in severe error (dark saturated blue, green, purple hues). The maximum error of the complete series for dark saturated blue is $53 \%$. While yellow and red hues show minimum error.

Image sensors that are used in the camera have limited color gamut, which results in inaccuracies in luminance of color sample with high chroma. Color gamuts of CMOS color sensors (saturated blues, and greens) limit measurements of Munsell color samples. Also, camera's color sensors are just approximations of the CIE color matching functions that add up to the error.

For deriving camera's response curve it is recommended to use grey cards and matte color checkers with the known reflectance and uniform illuminance. One of the samples with luminance determined from its reflectance and illuminance should serve as the reference. Surfaces of interest should use anchor with similar reflective properties. As paper suggests Munsell anchor N7.5 is recommended for the light surface elements, Munsell anchor N5 for medium reflectance elements (Moeck 2007). Among lighting environments that might be analyzed with the HDRI are non-uniform light sources, sky models, windows and luminaires, etc.

Study on the calibration factor's $(\mathrm{CF})$ variation over time for the HDR photography was investigated in research paper (Chung et al. 2010). Authors looked at the variations of $\mathrm{CF}$ in a single scene for various daylighting conditions. $\mathrm{CF}$ is determined by dividing physical luminance of a specific object in the scene by same region in the HDR image. In order to derive luminance of a physical scene this CF is multiplied by the region of the interest in the HDR image.

The X-Rite ColorChecker chart was used in the paper (figure 2-8). Electric lights in the classroom were turned off; measurements were conducted with stable clear and overcast sky conditions. 


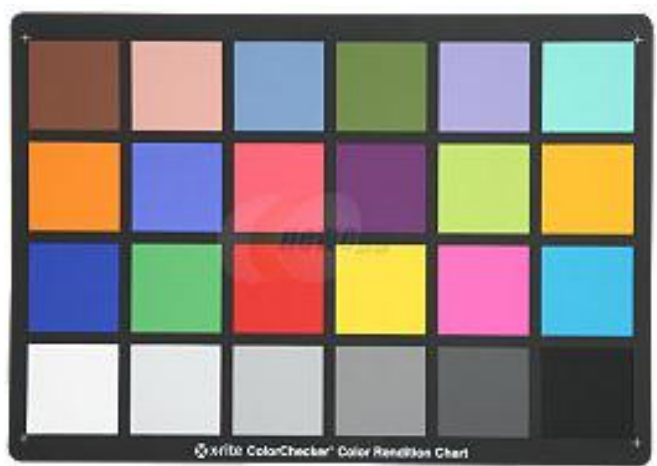

Figure 2-8. X-Rite ColorChecker chart that was used in (Chung et al. 2010) to analyze variations of calibration factor over time for the HDR photography in a daylit interior scene.

The HDR image was a product of nine LDR images in the range of $-4 \mathrm{EV}$ to 4EV. Authors used Aperture Priority Mode varying shutter speed. Then with hdrgen software camera responsive curve was derived (very dark and bright areas, large neutral grey and white smooth gradients in the scene were used). Then with the known responsive curve hdrgen can combine LDR images into a single HDRI.

Physical measurements of luminances were made by the calibrated luminance meter Minolta LS100 with 1 degree angle. Three measurements of each color in the chart were made before taking multiple exposures with the digital camera, and three after. Then these measurements were averaged and compared with the ones obtained from the HDRI.

Authors conducted experiments on 6 days at different times. Variation of mean vertical illuminance of the chart was in the range of 75 to 30000 lux. No direct sun was present in the scene during the experiments. Then CFs were computed and analyzed.

As a result, no influence of various lighting conditions was detected on HDRI's CF of any color. For any scene with a dominant color, one can achieve real luminance by multiplying the HDR luminance by $\mathrm{CF}_{\text {color }}$ of that color. 
Vignetting effect was analyzed and the proper correction ('digital filter') was applied in the study as well. The applied filter prevented luminance reduction at the corners of the image. In order to create data for such filter authors used white paper. They've measured luminances of nine points on the paper. And since the difference between measurements was less than 5\%, it was assumed to be matt. Then multiple photos of various exposures were taken. Vignetting effect was calculated by dividing every pixel of the photo by the value in the center of the HDRI (figure 2-9).

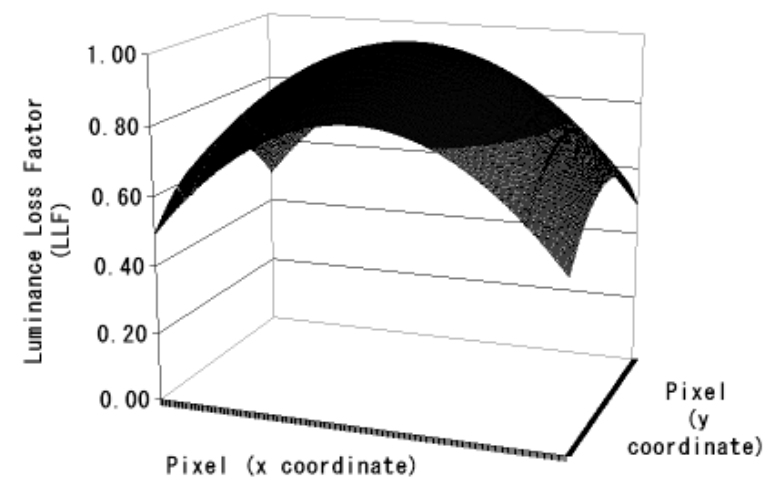

Figure 2-9. "Digital filter" for compensating luminance loss due to the vignetting effect in the study (Chung et al. 2010)

In "Improving the quality of high dynamic range images" (Cai et al. 2011) after applying vignetting correction and physical calibration HDR images were compared with each other on a quality of luminance acquisition. Very often camera with a wide-angle zoom or fisheye lens is used. These systems have many aberrations: vignetting effect (light falling off due to $\cos ^{4} \alpha$ at the periphery of an HDRI), diffraction of light (noticeable at small aperture), light scattering in the lens (depends on f-stop and the wavelength of light, and etc.), lens flare (CCD/CMOS sensors can't cover whole dynamic range), chromatic aberration (imperfect lens, or incorrect white balance of the camera), and radial distortion of the lens. The paper examines the dependence of quality of the HDR images on focal lengths and aperture sizes. The goal of the paper was to find an optimal combination of focal length and aperture size 
that lead to the least error: less vignetting, scattering and light diffraction at the lens. With large apertures light falls in the image periphery, and depth of field is narrower. Small apertures have diffraction issues. Aperture stop and light wavelength both influence light scattering in the lens. The number of EV is determined in the paper as well as appropriateness of using only one calibration factor for the entire scene was investigated.

This study for a specific camera and lens combination (Canon EOS 350D fitted with the Sigma lens 10-20mm F4-5.6 EX operating under fluorescent lighting) showed the following results. Higher quality HDR images are produced with larger apertures, while for medium apertures no significant difference was found. The smallest error obtained was at $\mathrm{f} / 5.6$ for each focal length 10,14 , and $20 \mathrm{~mm}$ under low or full fluorescent light levels. The maximum error obtained was for $\mathrm{f} / 22$.

HDR images photographed with a large aperture are not influenced by focal length. While with the small aperture quality of the HDR images of grey and colored samples can be improved with longer focal lengths. On the other hand, images taken with longer focal lengths decrease the quality of light-emitting surfaces. This tendency is reduced at smaller apertures. Noise in the camera image sensor contributes to the HDRI's error when black surfaces are photographed.

Quality of color, black, and luminous surfaces can be increased significantly using more EVs (starting from using 4-5 exposures). While for grey surfaces in the middle dynamic range (i.e. $4.8-212.9 \mathrm{~cd} / \mathrm{m}^{2}$ ) of scenes the quality starts to reach a plateau with almost no further improvement.

The targets of 12 greyscale patches on a foam board were placed on the front table, middle table, middle back table and on the back wall of the room. The quality of the HDR images of light-emitting surfaces and front grey targets can be 
significantly improved with higher ambient light level. But for the grey targets in the middle, middle back or back, neither for black nor for color targets the improvement of quality was found with the variation of the ambient light level.

Applying local or global physical calibration doesn't impact the quality of the HDR images. Although, local calibration might improve the accuracy as was suggested in the paper (Cai et al. 2011).

In (Moeck et al. 2006) authors give an explanation how to make an illuminance analysis from available HDR luminance maps. Luminances in the HDRI can be analyzed based on pixel's illuminance contribution at the camera. In order to obtain illuminance at the camera, one has to know luminance of every pixel and the solid angle. The meaningful grouping of pixels is a collection of pixels that represent visual tasks or objects in a scene like windows, walls, columns, etc. Technique proposed by the authors gives new opportunities to analyze illuminance in the space.

One has to be familiar with the limitations in the HDRI such as vignetting, luminance measurements of surfaces with low reflectances, and surfaces with high Munsell chroma. Authors used 5.1 Mpixel Nikon Coolpix 5400 (2003) with an F2.8F4.6 $4 \mathrm{X}$ optical zoom lens. The focal range of the lens is $5.8-24 \mathrm{~mm}$, which is equivalent to $28-116 \mathrm{~mm}$ for $35 \mathrm{~mm}$ film. All images were taken with the Matrix Auto White Balance with TTL control, using a Munsell N9.5 grey.

To obtain luminances from the luminance map authors used achromatic Munsell cards with values from two to nine. Physical measurements were done (1 degree angle of view luminance meter) for further comparison. Three cameras were compared: Nikon Coolpix 5400, Canon Digital Rebel and Olympus E1. All three performed similarly for grey scales with different Munsell values. The performance of these three cameras is much better than cameras without built in thermal noise 
suppression. But lower Munsell values are overestimated by the Nikon Coolpix, while the Olympus and Canon tend to underestimate them.

Luminance measurements of dark surfaces with low reflectance below N5 or $20 \%$ shows unreliable results for different lamp spectra (fluorescent $4100 \mathrm{~K}$, mercury vapor, and metal halide). Authors used automatic white balance. Authors suggest using spectral responsivity calibration to address this issue.

The results for Munsell hue and chroma are the following. Two different illuminance values of 478 lx and 88 lx were used (Sylvania Octron 4100K FO32/741 lamps). Saturated greens and blues didn't demonstrate reliable results. Although, high-to medium-reflectance blue and green hues with low chroma down to Munsell value N5 are within acceptable range. Warm colors with high Munsell values demonstrate reliable results. Fortunately, for a lot of applications this is not a major problem, since saturated dark hues are not often implemented in historic and modern buildings' interiors.

Illuminance can be expressed:

$$
\mathrm{E}=\int L_{\text {lum_meter }} \cos \beta d \omega
$$




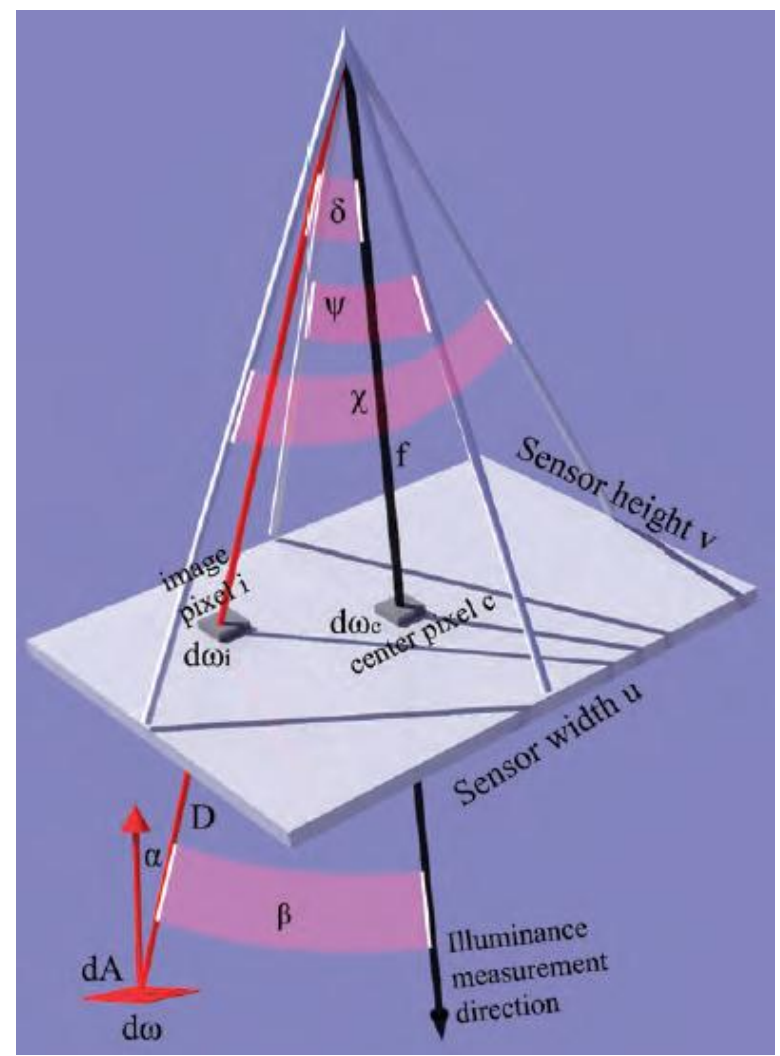

\section{Figure 2-10. Defining solid angles of central pixel d $\omega_{c}$, and any other} pixel i on a sensor $d \omega_{i}($ Moeck et al. 2006)

By defining solid angles (figure 2-10), one can obtain illuminance values from the luminance maps. Authors assessed the results on real experiments.

Table was uniformly lit with the indirect lighting. The illuminance was $480 \mathrm{~lx}$ (figure 2-11). There was a stack of five letter sized papers on a table. Top paper had twelve grids (0.0508 $\mathrm{m} \times 0.0762 \mathrm{~m}$ each). HDR image was obtained with the Nikon Coolpix 5400 camera out of twelve images with the shutter speeds varying from $1 / 2000$ to 1 second under maximum zoom. Luminance reference in the image was a Munsell N6 grey card with $30 \%$ reflectance. The HDR image was calibrated with the measured illuminance on the grey card. Minolta $1^{\circ}$ spot meter was used to measure the average luminance at the center of each grid on the white paper. Then the illuminance was calculated with:

$$
E=\sum_{i=1}^{12} \frac{L_{\text {lum_meter } \_} \times \cos \alpha i \times \cos \beta i \times \operatorname{Area}(i)}{D i^{2}}
$$


- area (i) is one of twelve $0.0508 \mathrm{~m} \times 0.0762 \mathrm{~m}$ sized patches on the paper with the uniform illuminance of 480 lx.

The calculated illuminance (equation 2.21) was $1.65 \mathrm{~lx}$.

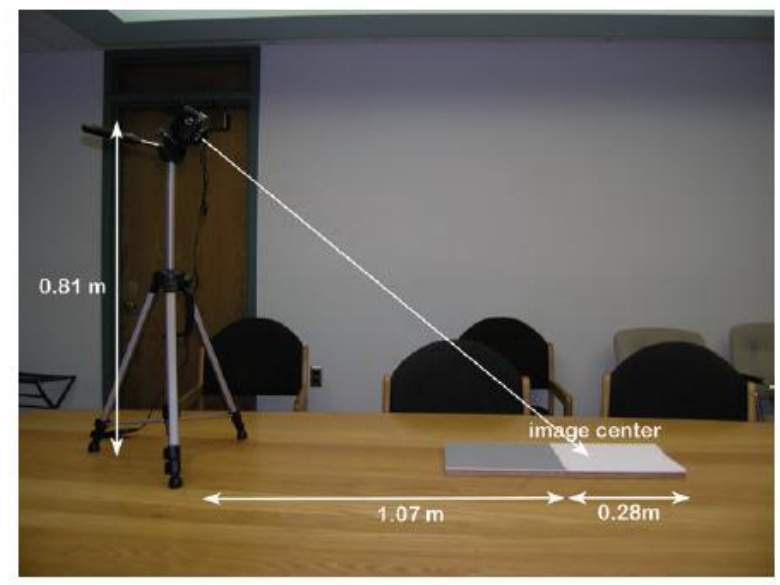

Figure 2-11. Set-up for assessing accuracy of obtaining illuminance values from the luminance map

The illuminance for all pixels from the HDR image was calculated using the following equation (figure 2-10):

$$
E=\int L_{i} \cos \delta d \omega i
$$

The sensors that were used in the camera (the CCD sensor size designated by $1 / 1.8^{\prime \prime}$ ) had dimensions of $u=7.18 \mathrm{~mm}$ and $v=5.32 \mathrm{~mm}$ (figure 2-10). The focal length was $24 \mathrm{~mm}$.

The illuminance of 1.72 lx was obtained from the HDRI (equation 2.22), which is within $5 \%$ error with the one obtained analytically from the equation 2.21 (table 2-1).

Table 2-1. Validation of illuminance calculation from a luminance map obtained with the HDRI (Moeck et al. 2006)

\begin{tabular}{|l|c|c|c|}
\hline & $\begin{array}{c}\text { Values Derived } \\
\text { Analytically Based } \\
\text { on Equation 2.21 }\end{array}$ & $\begin{array}{c}\text { Values Derived from } \\
\text { HDR Method using } \\
\text { Eq. 2.22 }\end{array}$ & $\begin{array}{c}\text { \% Error } \\
\text { Comparison }\end{array}$ \\
\hline Mean luminance (cd/m2) & 134.2972 & 131.0624 & 2.4087 \\
\hline Solid angle of paper (sr) & 0.0123 & 0.0131 & -6.8212 \\
\hline Illuminance due to paper (lux) & 1.6486 & 1.7180 & -4.2070 \\
\hline
\end{tabular}




\subsubsection{Studies of the HDRI technique capturing a natural scene and/or the sun}

In (Stumpfel et al. 2004) authors state that the HDR images can accurately capture natural illumination including the visible sun. Capturing the scene with the sun has two principal challenges. The first one is the breadth of dynamic range - the sun can be well over five times (or seventeen stops) brighter than the sky and clouds. So, varying only shutter speeds doesn't cover the whole range. And the second challenge is the absolute intensity of the sun, which is much brighter than what cameras are designed to capture.

Full dynamic range of the sun and sky can be preserved through careful selection of shutter speed, aperture size, and neutral density filter. The full range can be covered in seven exposures with a standard digital camera with a 12-bit linearresponse sensor. Work addresses calibration issues such as lens vignetting, infrared sensitivity, and spectral transmission of neutral density filter. Time-lapse renderings of a complex scene illuminated by a high-resolution, high dynamic range natural illumination environments demonstrate positive results.

Canon EOS-1Ds camera equipped with a 8mm Sigma fisheye lens pointing up was set on a roof with an unobstructed view of the horizon in all directions. Camera has an automatic exposure bracketing capability that varies exposure between 1 second and 1/8000 of a second. Camera yields only 14 stops (a factor of 16384). This is insufficient to capture the 17 stop range $(131,072 x)$ of a typical sunny sky. The usage of shutter speed longer than 2 seconds doesn't allow capturing the scene accurately due to the motion of the clouds.

Camera was controlled from a laptop. The developed program allows adjusting the shutter speed as well as the aperture of the camera. Varying two settings in the camera spanned the dynamic range of the sky. Lighting conditions of a natural 
environment change drastically with the sun position and weather over the course of the day. Images are analyzed as they are downloaded to a computer. Camera settings are changed if necessary.

It takes about 50 seconds to acquire and download a typical image sequence. To capture the sun directly a 3.0 neutral density (ND) filter was used, which reduces the amount of light passing through by a factor of 1000. ND filter caused a chromatic shift which had to be corrected.

The full range of the sunny sky can be covered in seven exposures with a standard digital camera with 12-bit linear-response sensor (figure 2-12).

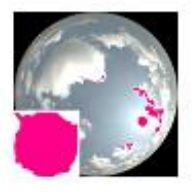

$\mathrm{f} / 4,1 \mathrm{~s}$

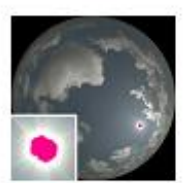

$\mathrm{f} / 4, \frac{1}{4} \mathrm{~s}$

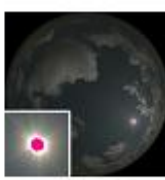

$\mathrm{f} / 4, \frac{1}{32} \mathrm{~s}$

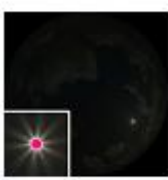

$\mathrm{f} / 16, \frac{1}{16} \mathrm{~s}$

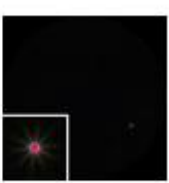

$\mathrm{f} / 16, \frac{1}{125} \mathrm{~s}$
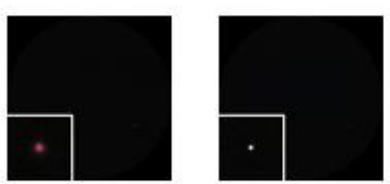

$\mathrm{f} / 16, \frac{1}{1000} \mathrm{~s} \quad \mathrm{f} / 16, \frac{1}{8000} \mathrm{~s}$

Figure 2-12. A typical HDR sequence spanning the 17 stops of the sky and the sun in 7 exposures

Obtaining faithful representation of sky luminance distributions is always a challenge due to the rapid changes of daylight environment (Inanici 2010). Sky scanners measure luminance at 145 points. These data are insufficient to represent actual sky conditions. There are 15 generic sky models specified by the CIE, but these models have the same disadvantages. The HDR imaging technique allows obtaining high resolution data for further analysis. Image-based lighting (IBL) is a visualization technique that allows using captured HDR images as light sources in a rendering process. IBL was not specifically developed for the lighting software, so it has to be evaluated for this application. Author acquired the HDR images of the sky dome. The main objectives of the paper were to develop technique on collecting high resolution 
data of image based sky luminances; application of the HDR image based sky models in lighting simulations; and assessment of image based sky models.

The HDRI was captured with Canon EOS 5D and fisheye lens Sigma $8 \mathrm{~mm}$ F3.5 EXDG that has $180^{\circ}$ angle of view and equi-angle projection properties. Camera was mounted on a tripod. White balance was set to daylight, ISO setting to 100, fixed aperture size of f/5.6 was used. Varying the shutter speed in manual exposure mode allowed capturing the sky. Shutter speed range is usually shortened under heavily cloudy sky since the dynamic range is reduced.

Using only one fixed aperture size does not allow capturing the sun. Therefore, author used two different apertures (f/4.0 and f/16) while varying shutter speeds. Neutral density filter Kodak Wratten 2 Optical Filter was used for the sequence.

About 10 images were taken with the aperture size of $f / 4$ and shutter speeds in the range of $15 \mathrm{~s}$ to $1 / 30$ ', and the aperture size of $\mathrm{f} / 16$ with shutter speeds in the range 1/15-1/8000 of a second. This approach allows recording sun in the sky dome (intermediate and clear). Twenty-two stop range corresponds to seven logarithmic units. Vignetting effect was taken into consideration in this work.

Specific calibration was used in this application. Usually sequence is calibrated with a single physical luminance measurement of a grey card obtained during the capturing process. But it is not practical to do such a calibration with the sky. Instead, horizontal illuminance is measured at the camera level.

The idea of IBL technique is to use captured sky dome as a light source in the software. Every pixel in the HDR image corresponds to physical value of luminance in $\mathrm{cd} / \mathrm{m}^{2}$, which contributes to the overall illuminance in a scene. All pixels of the obtained sky dome represent real world lighting conditions (figure 2-13). 

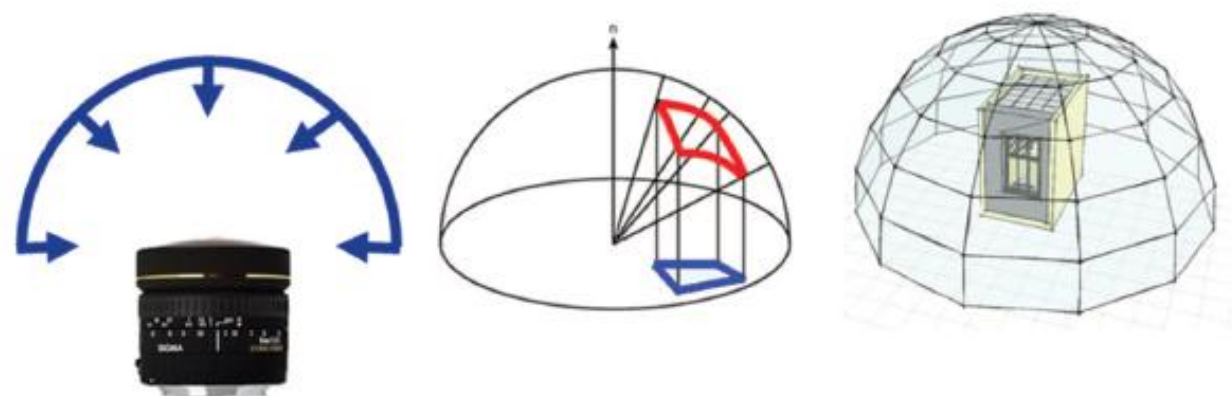

Figure 2-13. Fisheye projection and application of the HDR image as a light source in a computer simulation (Inanici 2010)

The discussed research included the following: the HDR images of the sky dome under various sky conditions and times were captured; at the same time the HDR images of the daylit office were obtained for measuring the luminance properties of the office; the CIE sky models and captured sky images were implemented in the same office with physically based rendering (PBR) and IBL techniques; comparison between captured and simulated lighting conditions were made.

The results show that image based sky models can provide reliable results for sky luminance distributions. This approach takes into account the influence of the surroundings. Also, the sun can be captured with the HDRI technique.

In (Konis et al. 2011) authors use the HDRI to assess innovative interior and exterior systems in comparison to the conventional methods under the real sun and sky conditions using existing visual comfort metrics.

The HDR images were obtained with Nikon 990 CCD camera with an equidistant fisheye lens (Nikon FC E8, 183º). Nine LDR images were taken in exposure-bracketed mode in $1 \mathrm{EV}$ steps (special script was written for the automated acquisition). Each image is 1536x1536 pixels. To acquire an HDRI hdrgen software was used. Authors applied "digital filter" to compensate for vignetting effect. 
Authors performed the test on accuracy of the HDRI under dynamic sky conditions.

Result of this paper show that if the HDRI are corrected for the lens light fall off and calibrated, luminance maps acquired with this technique show reliable results on average under dynamic sky conditions. But this holds true if change in global vertical illuminance during the acquisition of the HDRI is lower than 5\%. If it is not true, then these maps become less accurate.

One of the possible applications of the HDRI technique for analysis is represented in (Van et al. 2010). Authors study preference of occupants in a daylit office environment and the acceptance of luminance patterns using the HDR imaging. Lighting information from the HDRI was used to calculate Daylighting Glare Probability (DGP), Daylight Glare Index (DGI), and other metrics that used three luminance threshold analysis methods (figure 2-14). Luminance threshold analysis methods were the following:

\footnotetext{
Mean luminance of the scene;

Predetermined absolute luminance threshold, for example $2000 \mathrm{~cd} / \mathrm{m}^{2}$; - $\quad$ Mean luminance of the task.
} 


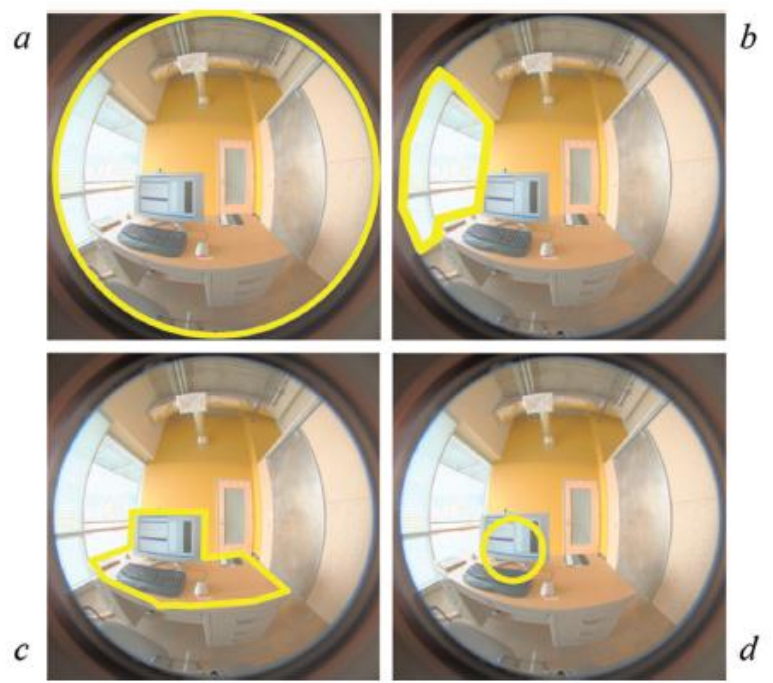

Figure 2-14. a - Mean luminance of the scene, $b$ - Predetermined absolute luminance threshold area, $c$ - Mean luminance task area (desk and monitor), d - Mean luminance task area (defined as subtended solid angle encompassing the screen and keyboard)

These methods were studied (along with additional metrics) for their ability to explain the assessments of luminance variability in the scenes under daylighting conditions (categories 'preferred' and 'just disturbing' were used).

Of the established methods, the most consistent and effective metrics to explain subjective responses were found to be: mean luminance of the task, mean luminance of the entire scene, and DGP using $2000 \mathrm{~cd} / \mathrm{m}^{2}$ as a glare source identifier.

One hundred and fifty candidate metrics were tested and revealed the most effective one, which is the 'mean luminance of the glare sources'. Glare sources were identified as 7 times more than the mean luminance of the task position in this metrics. Authors found out that DGP performed consistently better than DGI. See (Van et al. 2010) for further details see.

For glare assessment with the HDRI see (Bellia et al. 2009). 


\subsubsection{Outcome of literature review}

Many studies have proved that the HDRI technique is a promising tool and can be used for lighting measurements providing reliable high resolution luminance data, even though challenges exist.

The results of this research will contribute to the current state of knowledge on HDRI's implementation in lighting research and will allow expanding its capabilities. The investigation of the HDRI technique's ability to capture a bright light source (LED) with a narrow light distribution will be a valuable addition to the body of HDRI knowledge. 


\section{Chapter 3: Methodology}

\subsection{Experimental design}

The purpose of this research is to investigate whether the HDRI technique is capable of accurately capturing luminance of a bright light source (LED) with a narrow light distribution. To accomplish this goal a set of experiments was conducted. In order to assess the results of the HDRI technique, results had to be compared to the ones obtained with conventional methods of luminance measurement.

As was described in Section 2.1 there are three traditional ways to find out the luminance of a point in a certain direction:

1. To measure it with a luminance meter;

2. To derive it through the illuminance measurement and subsequent calculations;

3. Digital imaging photometer measurement;

Two methods of measuring luminance values were considered. The first one is to use specific close-up lens on a conventional luminance meter. Due to the small size of a light source (section 3.2.1) even with a luminance meter with one-third degree field of view, it is necessary to use a close-up lens (\#135, see appendix B) that would allow measuring luminance of an object as small as $1.8 \mathrm{~mm}$ in diameter.

The second one is through direct illuminance measurements in a constructed photometer. Luminance values were calculated through obtained luminous intensity curve and projected area of a single LED chip. Physical dimensions were measured and compared to the manufacturer's data.

For the definition of experimental settings for capturing luminance of a single LED chip with the HDRI, investigations of 2 cameras and 2 lenses were performed. Among the conducted experiments were the following. RCs for cameras were 
obtained in two different calibrating scenes (with and without a bright light source in the scene). Sequences of images were acquired with different shutter speed ranges. Vignetting effect was tested in various settings. After this preliminary stage of the experimental settings determination, series of images of a single LED chip in GE Garage luminaire were fused into the HDRI.

HDRI of a single LED chip was captured with CANON EOS 7D camera fitted with CANON zoom lens EF 28-105 mm 1:3.5-4.5 II USM. Neutral density filter NDA2-703-002 with $\boldsymbol{\tau}=0.0094$ was used on the lens to capture the LED chip. The range of shutter speeds was $1 / 8000$ to $30^{\prime \prime}$ at two aperture sizes (f/4.5 and f/16). Photograph histograms were checked in Photoshop CS5 to make sure that in the shortest exposure RGB values are lower than 228, and in the longest - higher than 27. RAW images were fused in hdrgen and calibrated with the hand-held measured luminance in Photosphere. The HDRI was analyzed and the luminance was compared to the one obtained with the traditional methods.

Among conducted experiments were the following:

Physical dimensions measurements;

Obtaining luminous intensity curve;

Luminance measurements with the luminance meter;

Luminance calculations from illuminance measurements in a created photometer;

Obtaining response curves for the different camera/lens combinations;

Vignetting experiments;

Uniformity of the Uniform Light Source (ULS);

HDRIs of LED with a ND filter;

Photoshop CS5 histograms check;

raw2hdr Perl script fusing; 
Photosphere analysis.

\subsection{Measurements of luminance of a single LED chip with traditional methods}

Two traditional methods of measuring luminance were considered.

The first one is to use conventional luminance meter (LS110 with one-third degree field of view) with a specific close-up lens (\#135). The close-up lens was used because of the small size of a light source (see 3.2.1). Luminance meter with a lens allows measuring the luminance of an object as small as $1.8 \mathrm{~mm}$ in diameter.

The second method is through direct illuminance measurements in a constructed photometer. Luminance values were calculated through the obtained luminous intensity curve and projected area of a single LED chip. Physical dimensions were measured and compared to the manufacturer's data.

\subsubsection{Measurements of a single LED chip's dimensions}

GE Lighting provided a luminaire for this research. It is GE Evolve ${ }^{\mathrm{TM}} \mathrm{LED}$ Garage fixture (EGMS-0-WL-N-60-P-C-10-white, see appendix B). CREE XP-E LEDs are used in this luminaire. The data on LEDs' sizes were obtained from the CREE website (figure 3-1 and appendix B), however, measurements were still performed. The only measurement that was impossible to acquire was the size of the square illuminating chip enclosed with the lens. For this dimension data was found on the CREE LED lighting website (figure 3-2).

Physical measurements were conducted with a micrometer. A single CREE XP-E LED has the following dimensions:

Square $3.480 \pm 0.254 \mathrm{~mm}(0.0137 \pm 0.0100) '$;

Silicone lens diameter $2.540 \pm 0.254 \mathrm{~mm}(0.10 \pm 0.01)$ '” 


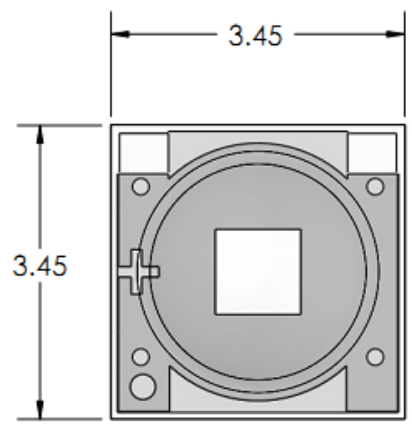

Top View

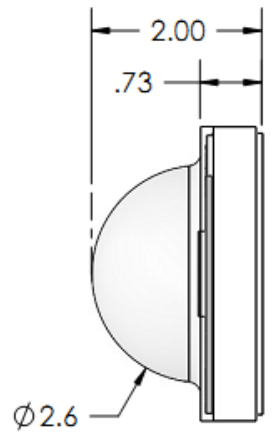

Side View

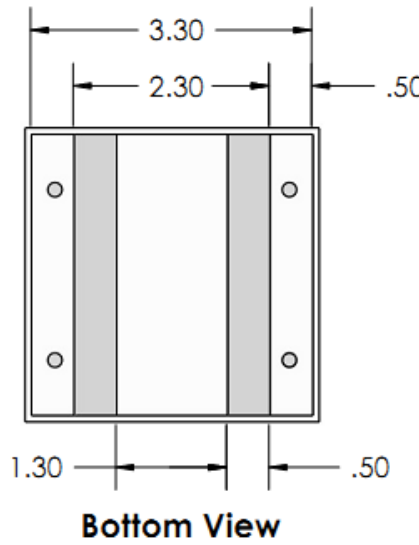

Bottom View

Figure 3-1. Cree ${ }^{\circledR}$ XLamp ${ }^{\circledR}$ XP-E LEDs
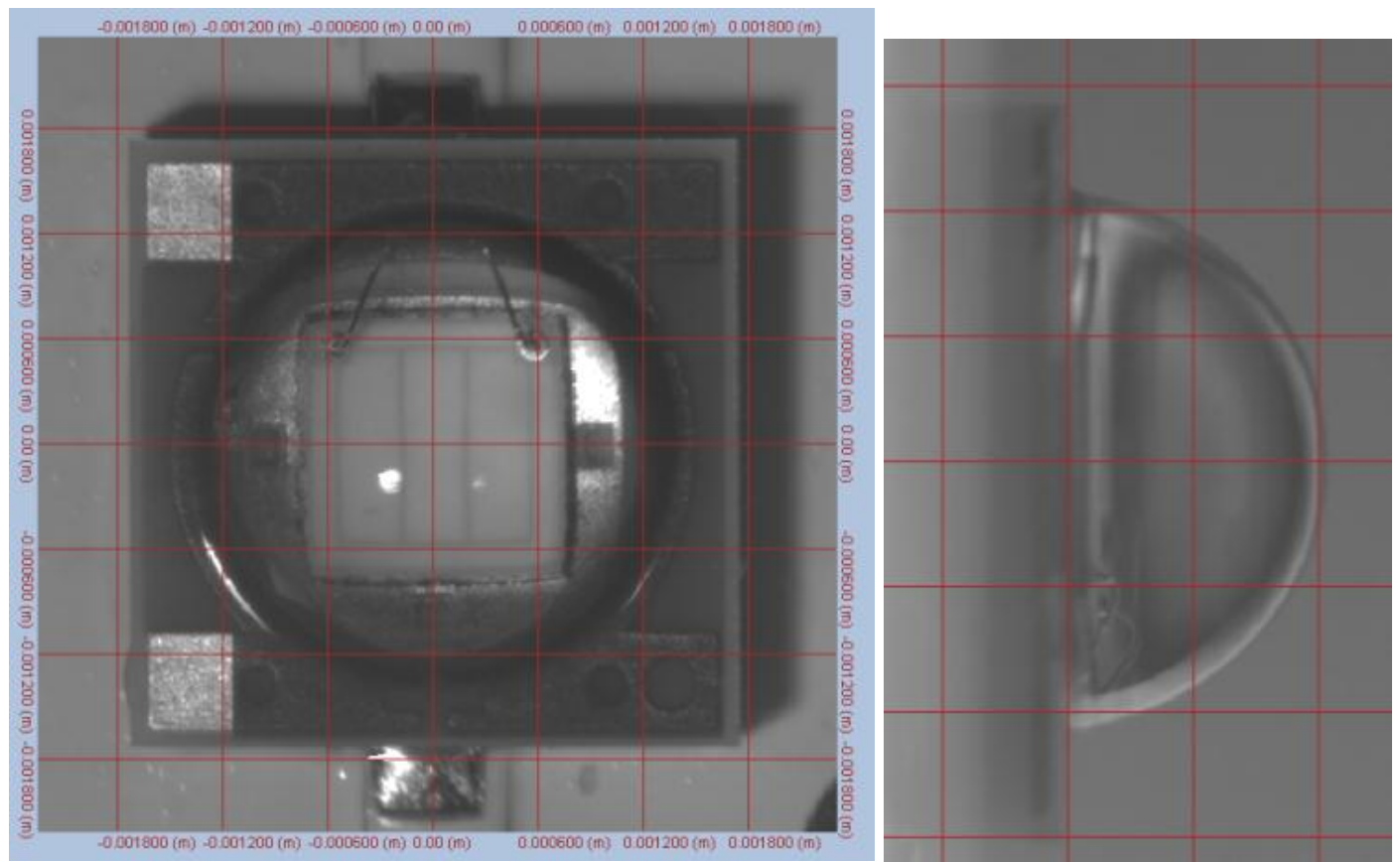

Figure 3-2. XP-E LED dimensions (CREE LED lighting)

According to figure 3-2 from the CREE LED lighting website, the side of a single LED chip is $1.4 \mathrm{~mm}(0.055$ ')

\subsubsection{Measuring luminance of a single LED chip in GE garage fixture} with the luminance meter

Goal: To obtain luminance of a single LED chip in GE garage fixture with the luminance meter (LS110). 
In order to assess HDRI's ability to perform photometric measurements of a bright light source with a narrow light distribution a conventional method of measuring luminance with the luminance meter was considered.

\subsubsection{Experimental settings}

For the purpose of obtaining luminance calibrated LS110 with the close-up lens \#135 was used (calibration certificate is in the appendix B). To account for the lens color correction a factor of 1.05 was set in the luminance meter. The close-up lens allows taking measurements as close as $1_{\text {minClose-up Lens }}=447 \mathrm{~mm}$ to an object to measure a minimum diameter of $d_{\text {Lmeterl }}=1.8 \mathrm{~mm}$ (figure 3-3, Appendix B).

Single LED chip is a very bright light source. When measuring its luminance value, the meter shows E0 mistake on the screen. It was found that luminance of the LED chip exceeds the measuring range of the luminance meter (which is 999900 $\mathrm{cd} / \mathrm{m}^{2}$ for LS110). Thus, neutral density filter had to be implemented. For this task NDA2-703-002 with $\boldsymbol{\tau}=0.0094$ was used. The transmittance of the filter was checked with the uniform luminance source (ULS) and LS110 luminance meter (the accuracy is within $2 \%$ ).

$$
\tau_{\text {measured }}=\frac{L_{\tau}}{L}=\frac{78}{8484}=0.0092
$$

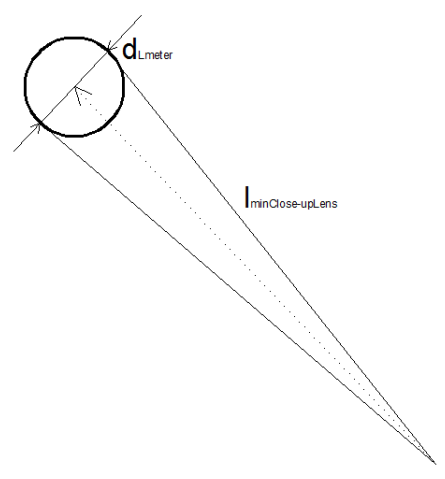

Figure 3-3. Minimum measured diameter of an object with a luminance meter LS110 and the close-up lens \#135 at a minimum distance 
The set-up for the experiment is shown on figure 3-4. The luminance meter was set on a tripod aimed at a single LED chip to the best ability. The distance from the LED chip to the sensor of the luminance meter was $(447 \pm 10) \mathrm{mm}$. Camera was aimed normal to the LED with an error of $(0 \pm 5)^{\circ}$. When measurements were taken, there was no ambient light in the room.
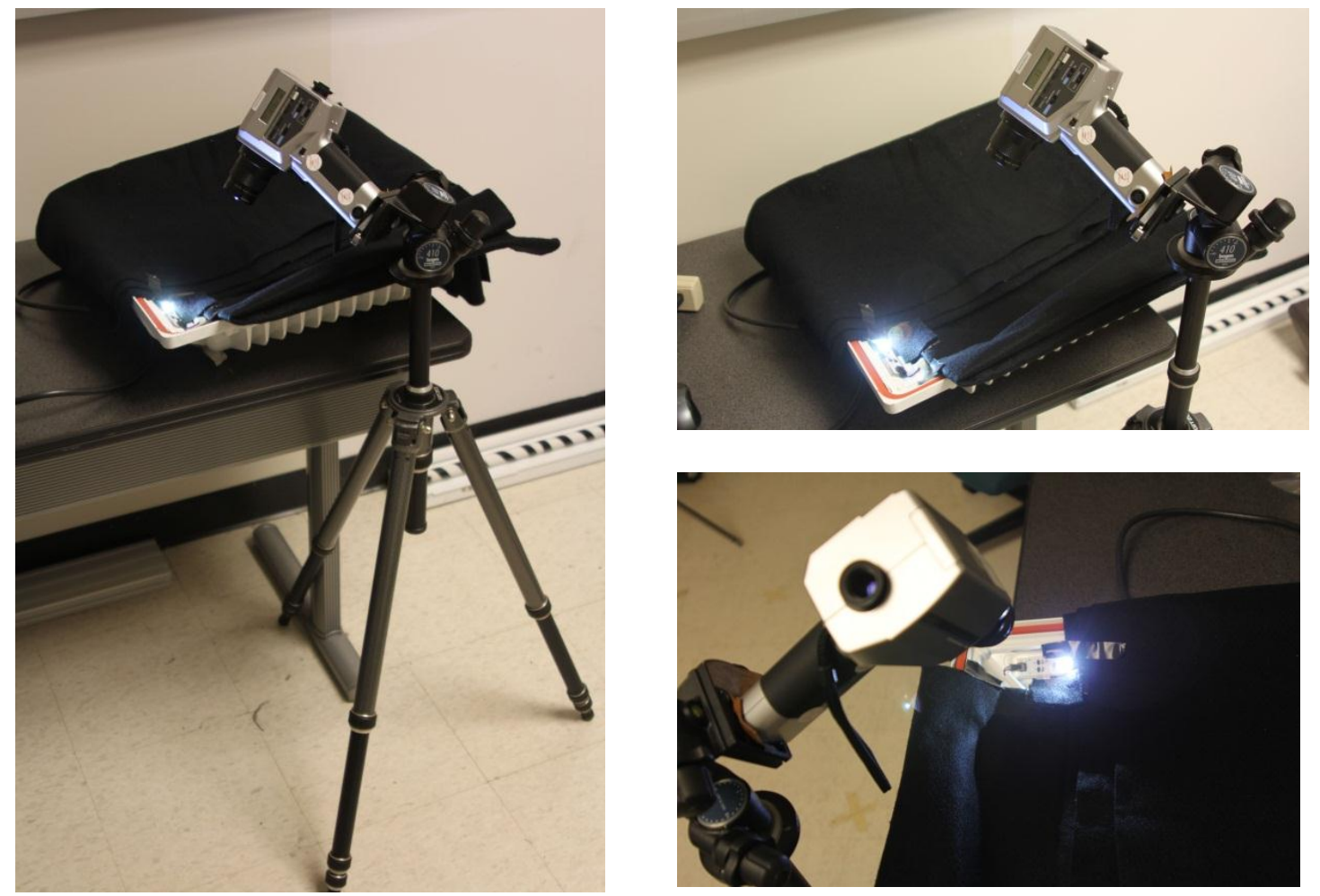

Figure 3-4. The set-up for the luminance meter measurement of a single LED chip

Challenges in aiming the luminance meter at a single LED chip are:

1. A very small size of the LED chip (refer to section 3.2.1). To provide an accurate measurement measured area of the object should be bigger than the acceptance angle of the luminance meter within which it averages the reading (figure 3-3). Since the difficulty in aiming the luminance meter exists, the right approach is to search for the peak value. Besides aiming at the LED chip through the eye piece, looking at the luminance readings on the side of the luminance meter while adjusting the tripod is a good strategy to use; 
2. A very high luminance value. Using NDA2-703-002 filter is critical for measuring purposes as well as for aiming purposes through the eye piece.

The highest obtained luminance value is $6.97 * 10^{6} \mathrm{~cd} / \mathrm{m}^{2}$.

Outcome: It's impossible to get a reliable luminance measurement of a single LED chip with the LS110 luminance meter even with the close-up lens (\#135).

3.2.2.2 Analyzing the overlay of a single LED chip area and the measuring area of LS110 luminance meter

If the luminance meter is aimed perfectly at the center of the LED chip, it still does not cover LED's whole area (see figure 3-5).

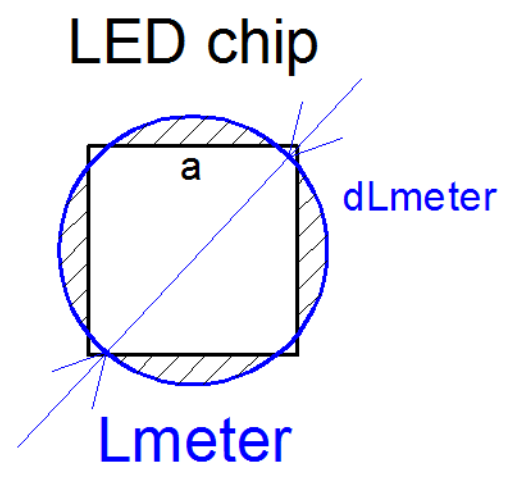

Figure 3-5. Metering circle of LS110 luminance meter and the area of a single LED chip. Relative sizes are to scale

Minimum metering area of the luminance meter at a minimum distance (see figure 3-3):

$\mathrm{A}_{\text {Lmeter }}=\pi *\left(\frac{d}{2}\right)^{2}=\pi *\left(\frac{1.8}{2}\right)^{2}=2.54 \mathrm{~mm}^{2}$

Area of a single LED chip (section 3.2.1):

$\mathrm{A}_{\text {LEDchip }}=\mathrm{a}^{2}=1.4^{2}=1.96 \mathrm{~mm}^{2}$

Compute luminance meter measuring area as $100 \%$, the overlay of the areas $(\mathrm{x}, \%)$ :

$2.54 \mathrm{~mm}^{2}-100 \%$

$1.96 \mathrm{~mm}^{2}-\mathrm{x}$ 
$x=77 \%$

The emitting surface of the LED covers only $77 \%$ of the measurement target of the luminance meter if aligned. Otherwise, the average luminance results in even a lower value, since more of the non-illuminating area around the LED chip is captured by a measuring circle of the luminance meter.

Outcome: Luminance meter measures LED chip with its surroundings. It explains the unreliable luminance measured with LS110 and the close-up lens (\#135).

\subsubsection{Luminance calculation through the luminous intensity curve and area}

measurements

Goal: To obtain average luminance of a single LED chip in GE garage fixture through calculations from illuminance measurements.

One of the ways to get luminance of a light source is to calculate it through direct illuminance measurements. LED is a point light source, therefore the inverse cosine square law can be used:

$$
E=\frac{I * \cos \propto}{D^{2}} \quad(3.4), \text { where }
$$

D - Distance from the light source (LED) to the sensor of the illuminance meter (T-10).

Direct illuminance measurement allows the calculation of luminous intensity data of a point light source. Plane of the illuminance meter sensor in the created photometer is perpendicular to a line from the LED chip to the meter at all times $(\cos \alpha=1)$, then from (3.4):

$$
I=E * D^{2}
$$

We assume LED chip is a uniform light source, therefore average luminance is:

$$
L=\frac{I}{A_{\text {projected }}}
$$


From (3.6):

$I=L * A_{\text {projected }}$

Projected area of the square LED chip:

$A_{\text {projected }}=a^{2} * \cos \alpha \quad$ (3.8), where

a - side of square LED chip emitting surface;

$\alpha$ - angle between normal to the LED and photometer.

And, finally, luminance can be calculated if (3.6) is substituted with (3.5) and (3.8):

$$
L=\frac{I}{A_{\text {projected }}}=\frac{E * D^{2}}{a^{2} * \cos \alpha}
$$

\subsubsection{Description of a created photometer}

For the purpose of measuring direct illuminance from a single LED chip the photometer based on the apparatus used for the previous research at PKI was created (Eble-Hankins et al. 2009).

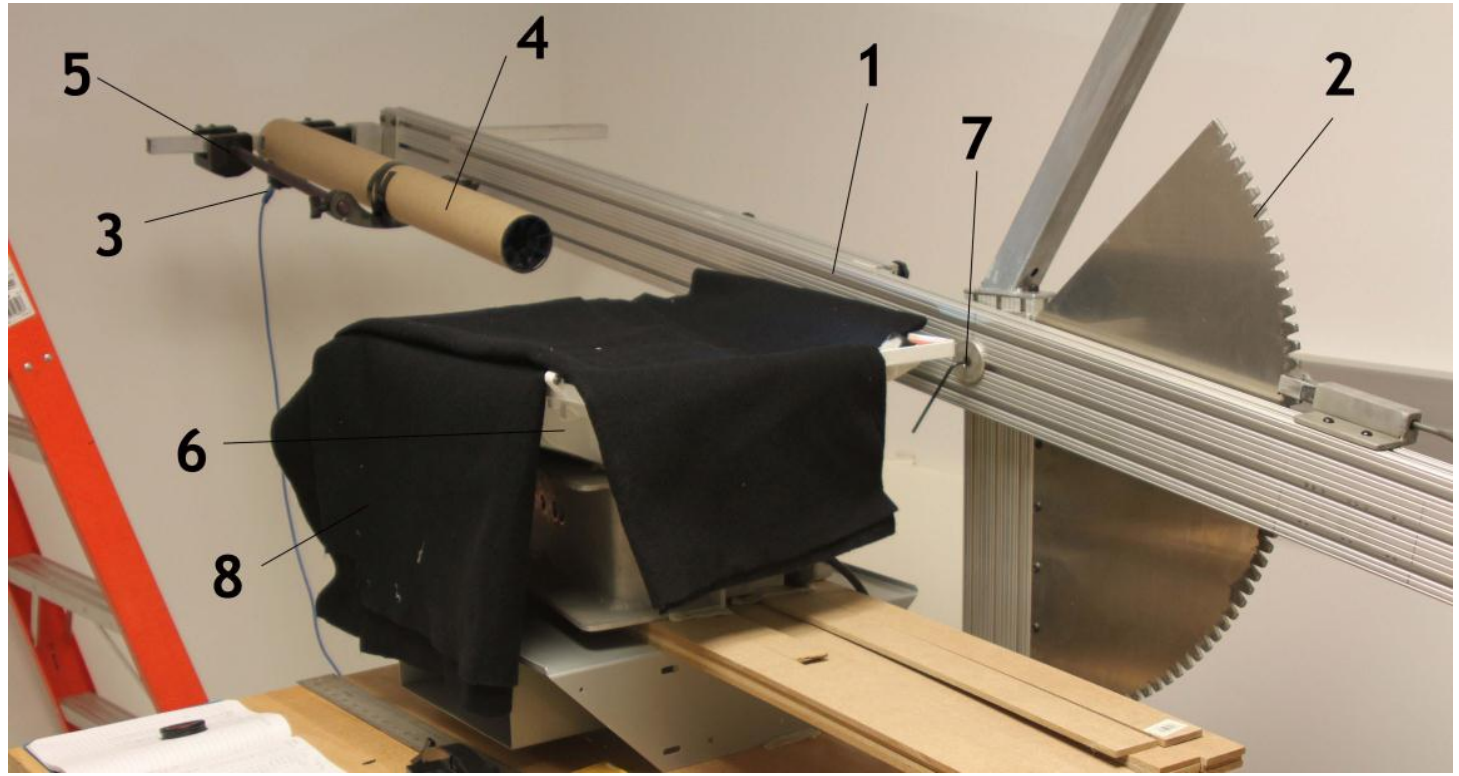

Figure 3-6. Created photometer for measuring direct illuminance from a light source

80/20 Inc.'s extruded aluminum modular framing system was used for the construction of the apparatus. Created photometer (figure 3-6) has an adjustable- 
length arm (1) that can be rotated via a gear in 2.5 degree increments (2) from 90 degrees above horizontal to 30 degrees below horizontal. Illuminance meter (3) was placed on the arm of the photometer. For this purpose hole was drilled in the arm of the photometer and bolt was used to have a rigid and stable connection. The tube (4) was placed along the operable arm covering the illuminance meter. One side of the tube was cut in such a way so that it can be mounted on the arm of the photometer and cover the sensor (figure 3-7). To aim the tube at the LED the adjustable rest (5) was used. The aiming is done visually when illuminance meter is taken out (figure 3-8). The luminaire (6) was placed on a rigid table with adjustable legs. The cover of the luminaire was taken off (figure 3-9). One of the fixtures' LED arrays was aligned with the pivot point (7) of the apparatus, so that the distance from the LED chip to the illuminance meter sensor is constant every time the angle of the photometer is changed. In order to eliminate the light from all other LEDs in arrays dark nontransparent fabric (8) was used. At the same time fabric doesn't completely cover heat dissipation parts of the luminaire. 

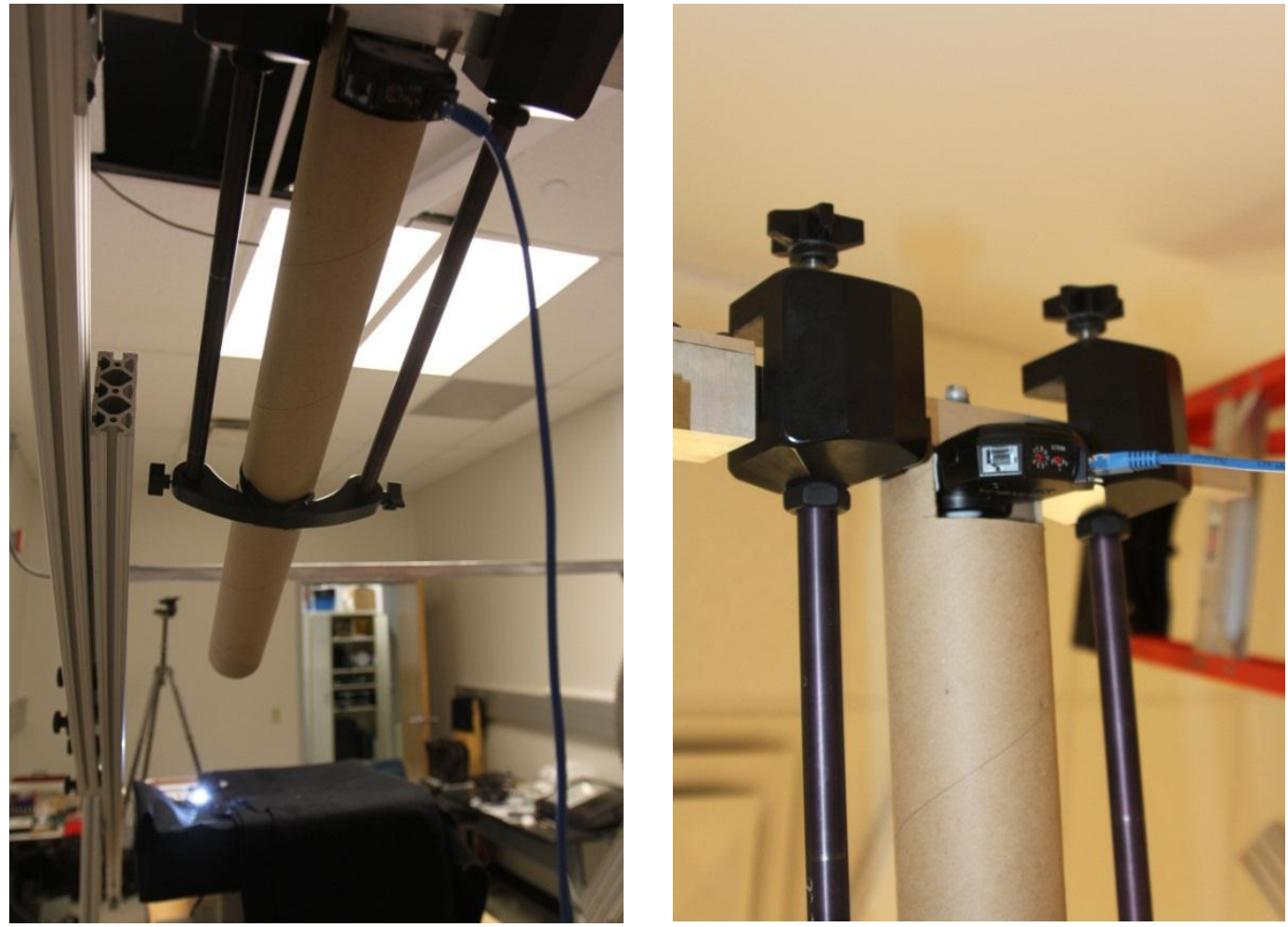

Figure 3-7. Tube covering the illuminance meter
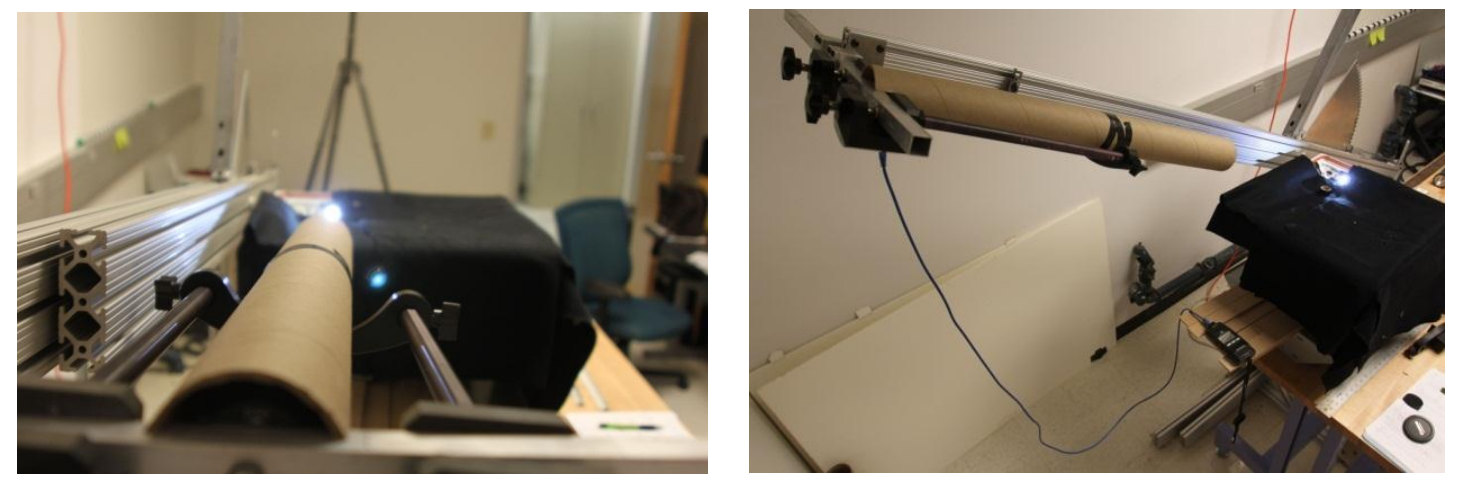

Figure 3-8. Aiming at a light source 


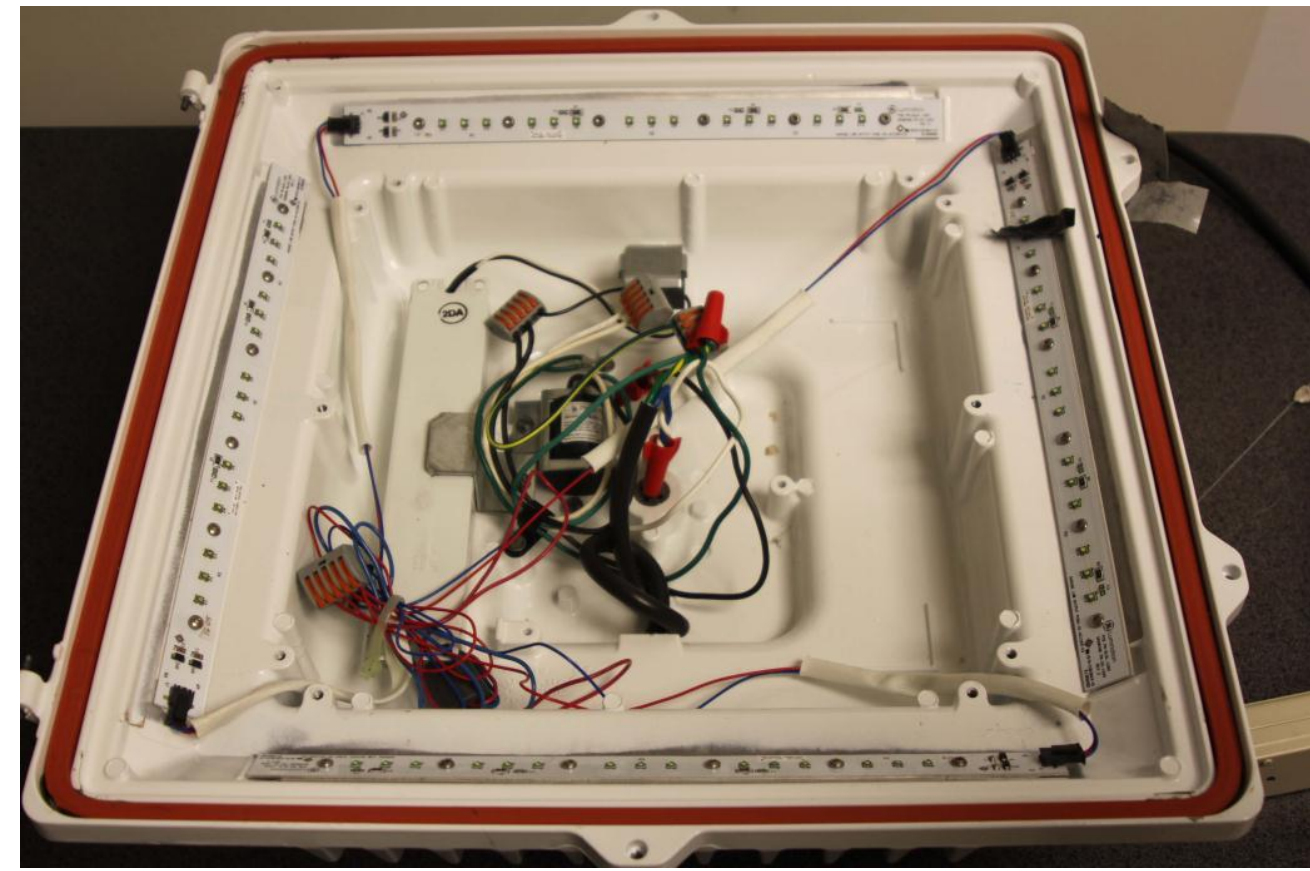

Figure 3-9. GE Garage Luminaire without the cover and reflectors

Multiple reflections in the tube add up to the indirect illuminance component.

In order to have direct illuminance measurement only, it's necessary to minimize all reflections reaching the illuminance meter. For this purpose a cardboard tube was created in a special way (figure 3-10). It is $8.255 \mathrm{~cm}\left(3 \frac{1 / 4}{4}\right.$, C) diameter, $86 \mathrm{~cm}$ (34', A) height cardboard tube. In order to eliminate reflections inside the tube two baffles were placed inside. One is located at the far end of the tube (its diameter is $8.255 \mathrm{~cm}\left(3 \frac{1}{4} ",, C\right)$ with the aperture of $2.86 \mathrm{~cm}(11 / 8 ”, D)$ in the middle, the other one is located $27.94 \mathrm{~cm}\left(11^{\prime \prime}, \mathrm{B}\right)$ away from the sensor, so all reflections can be absorbed before they reach the illuminance meter's sensor. On the other hand, the areas of the apertures were picked in such a way that the diameter of baffles is big enough for the whole sensor area to be illuminated; this would give the correct measurement. 


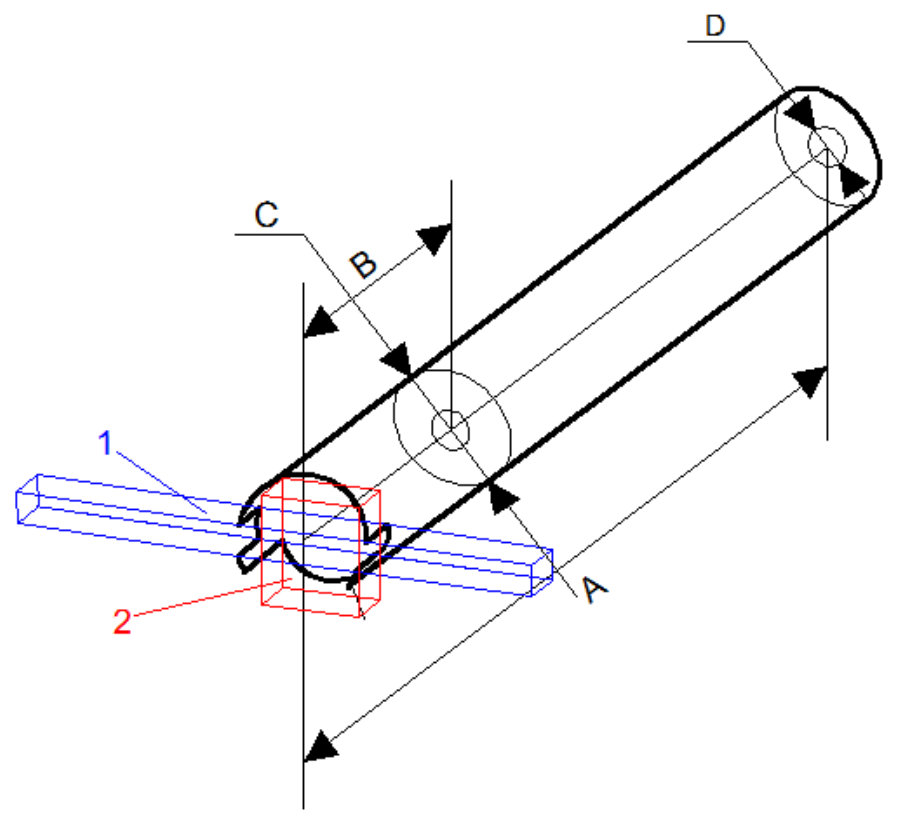

A $-86 \mathrm{~cm}$

B $-27.94 \mathrm{~cm}$

C $-8.255 \mathrm{~cm}$

D $-2.86 \mathrm{~cm}$

1 - operable arm

2 - illuminance meter

Figure 3-10. Dimensions of the created cardboard tube

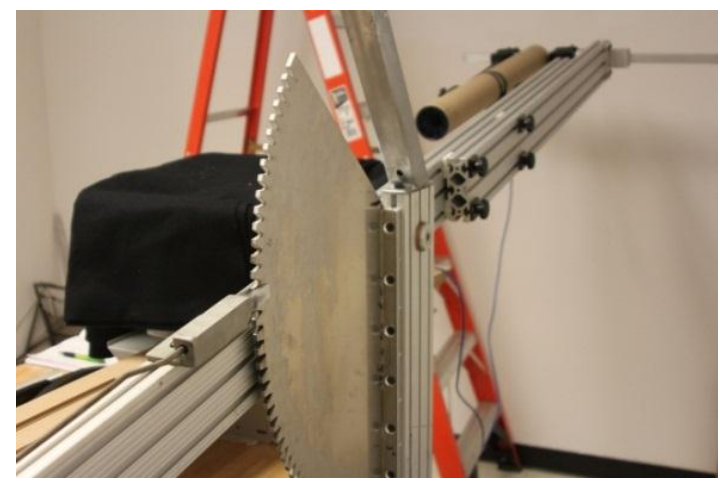

Figure 3-11. Changing gear in 2.5 degree increments

Measurements of illuminance were taken twice moving photometer both directions every 2.5 degrees (figure 3-11). Then measurements were averaged (table 3-1). There was no ambient light in the room during the measurements. To make sure LED chip was aimed at the center of the tube's aperture the aiming at LED was checked every 15 degrees. Re-aiming was performed when necessary.

Every array in the luminaire is angled (figure 3-12), thus the normal angle to the array from the horizontal had to be determined. Goniometer was used to find the 
angle, which is 47.5 degrees from horizontal. Then angles of the photometer were recalculated, so that normal to the LED is $0^{\circ}$.

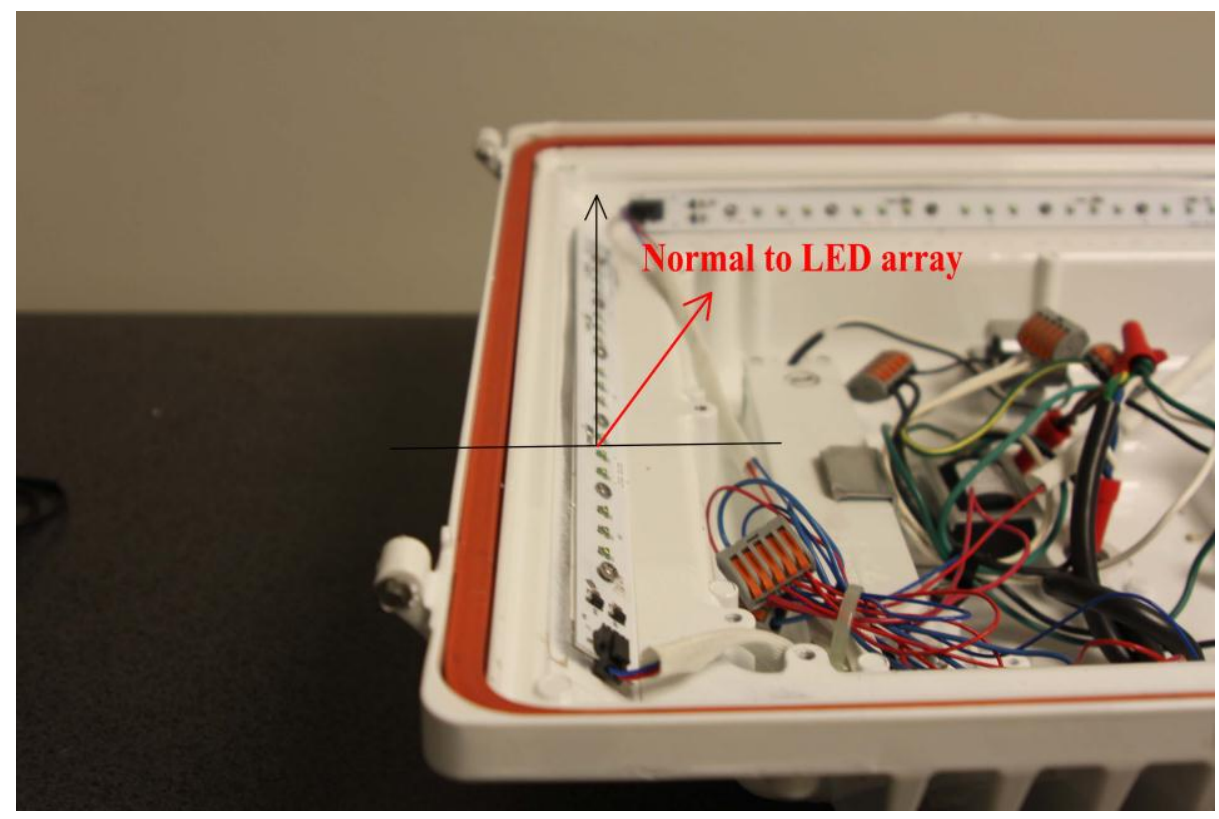

Figure 3-12. Normal to the LED array

After obtaining illuminance values, luminous intensity curve (figure 3-13) and luminance values of the LED were calculated using formulas 3.5 and 3.9 (the results are in the table 3-1).

An experiment of the variability of the light output over time of a single LED was conducted to make sure the output of the LED was constant. Both output and source voltage were measured in a 7-hour period (appendix C).

\subsubsection{Analysis of direct illuminance measurements from a single LED chip}

Table 3-1 has all measured illuminance levels and calculated luminance values.

Measured distance between the illuminance meter sensor and a single LED chip used in equation 3.9 is $\mathrm{D}=1.55 \mathrm{~m}(61$ ') $)$.

The LED chip has the same luminance values with 5\% error within the investigated angles (-40 to $42.5^{\circ}$ ) (table 3-1, figure 3-14). 
Such a drastic difference in calculated average luminance value $\left(19.44 * 10^{6}\right.$ $\left.\mathrm{cd} / \mathrm{m}^{2}\right)$ and value measured with the luminance meter $\left(\mathbf{6 . 9 7} * 10^{\mathbf{6}} \mathrm{cd} / \mathrm{m}^{2}\right)$ has to be explained (see sections 3.2.2.2 and 3.4.5.2). 
Table 3-1. Measurements of direct illuminance from a single LED and calculations of luminous intensity, projected area and luminance values (measured on the 2/3/2012, 3-5:30pm)

\begin{tabular}{|c|c|c|c|c|c|c|c|}
\hline \multicolumn{3}{|c|}{ Measured values } & \multirow{2}{*}{$\begin{array}{l}\frac{\text { Redefined }}{\text { angle }} \\
\text { Angles from } \\
\text { the normal to } \\
\text { a single LED } \\
\text { chip, degrees }\end{array}$} & \multicolumn{4}{|c|}{ Calculated values } \\
\hline $\begin{array}{c}\text { Angles of } \\
\text { photometer, } \\
\text { degrees }\end{array}$ & $\begin{array}{c}\text { E, lux } \\
\text { (from } \\
7.5 \text { to } \\
90^{\circ} \text { ) }\end{array}$ & $\begin{array}{c}\text { E, lux } \\
\text { (from } \\
90 \text { to } \\
7.5^{\circ} \text { ) }\end{array}$ & & $\mathbf{E}_{\text {average, }}$ lux & $\mathbf{I}(\alpha), \mathbf{c d}$ & $\begin{array}{l}\text { Projected area of } \\
\text { the LED chip, } \mathbf{m}^{2}\end{array}$ & $\mathrm{~L}$ of a chip, $\mathrm{cd} / \mathrm{m}^{2}$ \\
\hline 7.5 & 13.09 & 12.51 & -40 & 12.8 & 29.36 & $1.50 * 10^{-6}$ & $19.55 * 10^{6}$ \\
\hline 10 & 13.68 & 13.08 & -37.5 & 13.38 & 30.69 & $1.55 * 10^{-6}$ & $19.73 * 10^{6}$ \\
\hline 12.5 & 14.14 & 13.51 & -35 & 13.83 & 31.71 & $1.61 * 10^{-6}$ & $19.75^{*} 10^{6}$ \\
\hline 15 & 14.44 & 13.79 & -32.5 & 14.12 & 32.37 & $1.65 * 10^{-6}$ & $19.58 * 10^{6}$ \\
\hline 17.5 & 14.67 & 13.99 & -30 & 14.33 & 32.86 & $1.70 * 10^{-6}$ & $19.36 * 10^{6}$ \\
\hline 20 & 14.99 & 14.3 & -27.5 & 14.65 & 33.59 & $1.74 * 10^{-6}$ & $19.32 * 10^{6}$ \\
\hline 22.5 & 15.26 & 14.59 & -25 & 14.93 & 34.23 & $1.78 * 10^{-6}$ & $19.27 * 10^{6}$ \\
\hline 25 & 15.47 & 14.83 & -22.5 & 15.15 & 34.75 & $1.81 * 10^{-6}$ & $19.19 * 10^{6}$ \\
\hline 27.5 & 15.69 & 15.03 & -20 & 15.36 & 35.23 & $1.84 * 10^{-6}$ & $19.13 * 10^{6}$ \\
\hline 30 & 15.86 & 15.21 & -17.5 & 15.54 & 35.63 & $1.87 * 10^{-6}$ & $19.06 * 10^{6}$ \\
\hline 32.5 & 16.03 & 15.31 & -15 & 15.67 & 35.94 & $1.89 * 10^{-6}$ & $18.98 * 10^{6}$ \\
\hline 35 & 16.35 & 15.59 & -12.5 & 15.97 & 36.63 & $1.91 * 10^{-6}$ & $19.14 * 10^{6}$ \\
\hline 37.5 & 16.45 & 15.845 & -10 & 16.15 & 37.03 & $1.93 * 10^{-6}$ & $19.18 * 10^{6}$ \\
\hline 40 & 16.59 & 16.04 & -7.5 & 16.32 & 37.42 & $1.94 * 10^{-6}$ & $19.26 * 10^{6}$ \\
\hline 42.5 & 16.68 & 16.12 & -5 & 16.4 & 37.61 & $1.95 * 10^{-6}$ & $19.26 * 10^{6}$ \\
\hline 45 & 16.69 & 16.14 & -2.5 & 16.42 & 37.64 & $1.96 * 10^{-6}$ & $19.22 * 10^{6}$ \\
\hline 47.5 & 16.59 & 16.2 & $\mathbf{0}$ & 16.40 & 37.60 & $1.96 * 10^{-6}$ & $19.18 * 10^{6}$ \\
\hline 50 & 16.55 & 16.21 & 2.5 & 16.38 & 37.57 & $1.96 * 10^{-6}$ & $19.18 * 10^{6}$ \\
\hline 52.5 & 16.52 & 16.17 & 5 & 16.35 & 37.49 & $1.95 * 10^{-6}$ & $19.20 * 10^{6}$ \\
\hline
\end{tabular}




\begin{tabular}{|c|c|c|c|c|c|c|c|}
\hline 55 & 16.48 & 16.13 & 7.5 & 16.31 & 37.39 & $1.94 * 10^{-6}$ & $19.24 * 10^{6}$ \\
\hline 57.5 & 16.43 & 16.08 & 10 & 16.26 & 37.28 & $1.93 * 10^{-6}$ & $19.31 * 10^{6}$ \\
\hline 60 & 16.31 & 16.015 & 12.5 & 16.16 & 37.06 & $1.91 * 10^{-6}$ & $19.37 * 10^{6}$ \\
\hline 62.5 & 16.09 & 15.9 & 15 & 16.00 & 36.68 & $1.89 * 10^{-6}$ & $19.38 * 10^{6}$ \\
\hline 65 & 15.95 & 15.78 & 17.5 & 15.87 & 36.38 & $1.87 * 10^{-6}$ & $19.47 * 10^{6}$ \\
\hline 67.5 & 15.74 & 15.58 & 20 & 15.66 & 35.91 & $1.84 * 10^{-6}$ & $19.50 * 10^{6}$ \\
\hline 70 & 15.55 & 15.4 & 22.5 & 15.48 & 35.49 & $1.81 * 10^{-6}$ & $19.60 * 10^{6}$ \\
\hline 72.5 & 15.3 & 15.15 & 25 & 15.23 & 34.92 & $1.78 * 10^{-6}$ & $19.66 * 10^{6}$ \\
\hline 75 & 14.98 & 14.78 & 27.5 & 14.88 & 34.12 & $1.74 * 10^{-6}$ & $19.63 * 10^{6}$ \\
\hline 77.5 & 14.73 & 14.5 & 30 & 14.62 & 33.52 & $1.70 * 10^{-6}$ & $19.75 * 10^{6}$ \\
\hline 80 & 14.44 & 14.24 & 32.5 & 14.34 & 32.89 & $1.65 * 10^{-6}$ & $19.90 * 10^{6}$ \\
\hline 82.5 & 14.11 & 13.91 & 35 & 14.01 & 32.13 & $1.61 * 10^{-6}$ & $20.01 * 10^{6}$ \\
\hline 85 & 13.58 & 13.39 & 37.5 & 13.49 & 30.93 & $1.55 * 10^{-6}$ & $19.89 * 10^{6}$ \\
\hline 87.5 & 13.02 & 12.84 & 40 & 12.93 & 29.65 & $1.50 * 10^{-6}$ & $19.75^{*} 10^{6}$ \\
\hline \multirow[t]{5}{*}{90} & 12.73 & 12.55 & 42.5 & 12.64 & 28.99 & $1.45 * 10^{-6}$ & $20.06 * 10^{6}$ \\
\hline & & & & & & $\begin{array}{c}\text { Maximum } \\
\text { luminance value, } \\
\mathrm{cd} / \mathrm{m}^{2}\end{array}$ & $20.06 * 10^{6}$ \\
\hline & & & & & & $\begin{array}{c}\text { Minimum } \\
\text { luminance value, } \\
\mathrm{cd} / \mathrm{m}^{2}\end{array}$ & $18.98 * 10^{6}$ \\
\hline & & & & & & Error, \% & 5.37 \\
\hline & & & & & & $\begin{array}{c}\text { Average } \\
\text { luminance value, } \\
\mathrm{cd} / \mathrm{m}^{2}\end{array}$ & $19.44 * 10^{6}$ \\
\hline
\end{tabular}




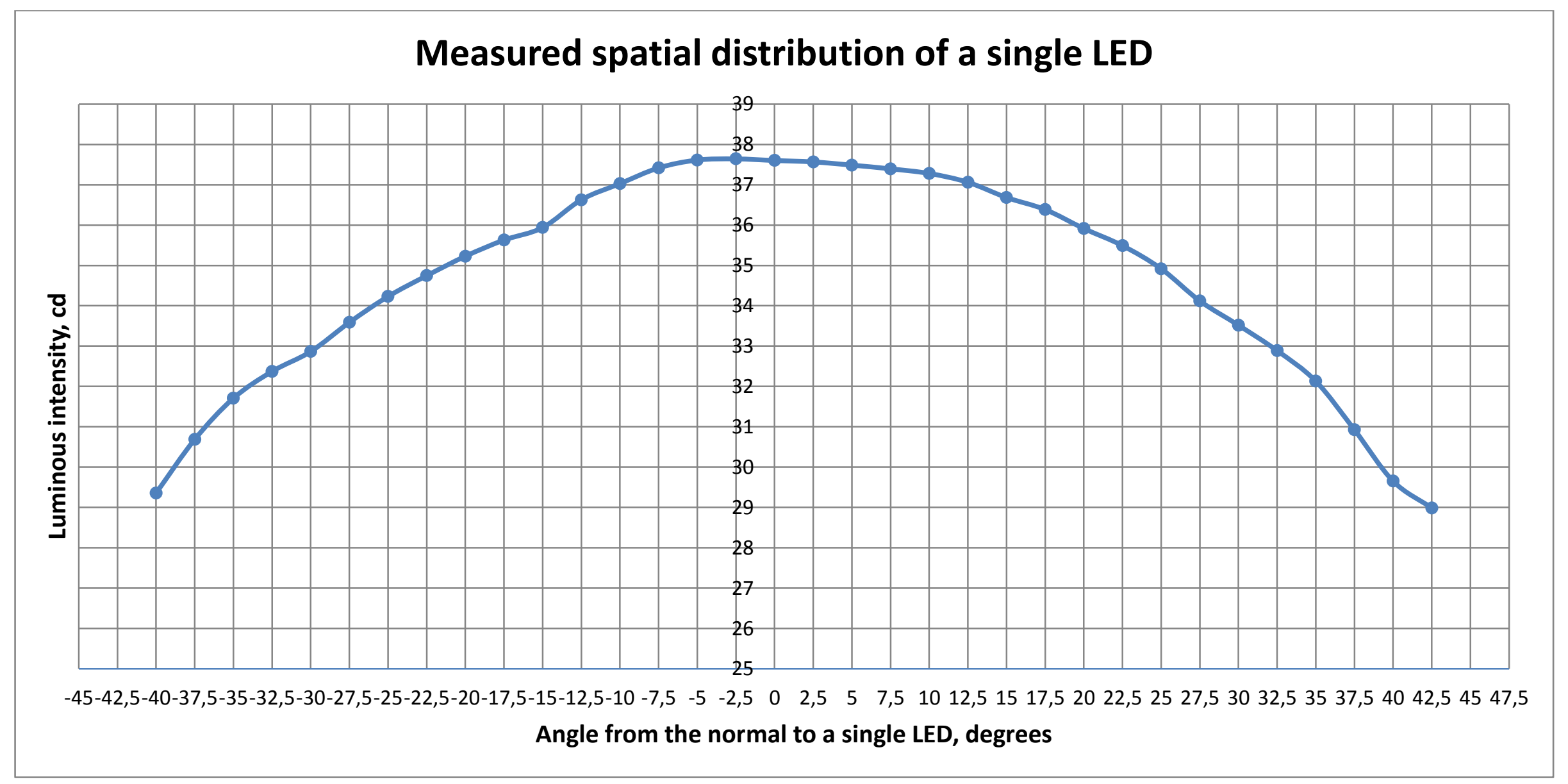

Figure 3-13. Luminous intensity curve of a single LED chip, cd 


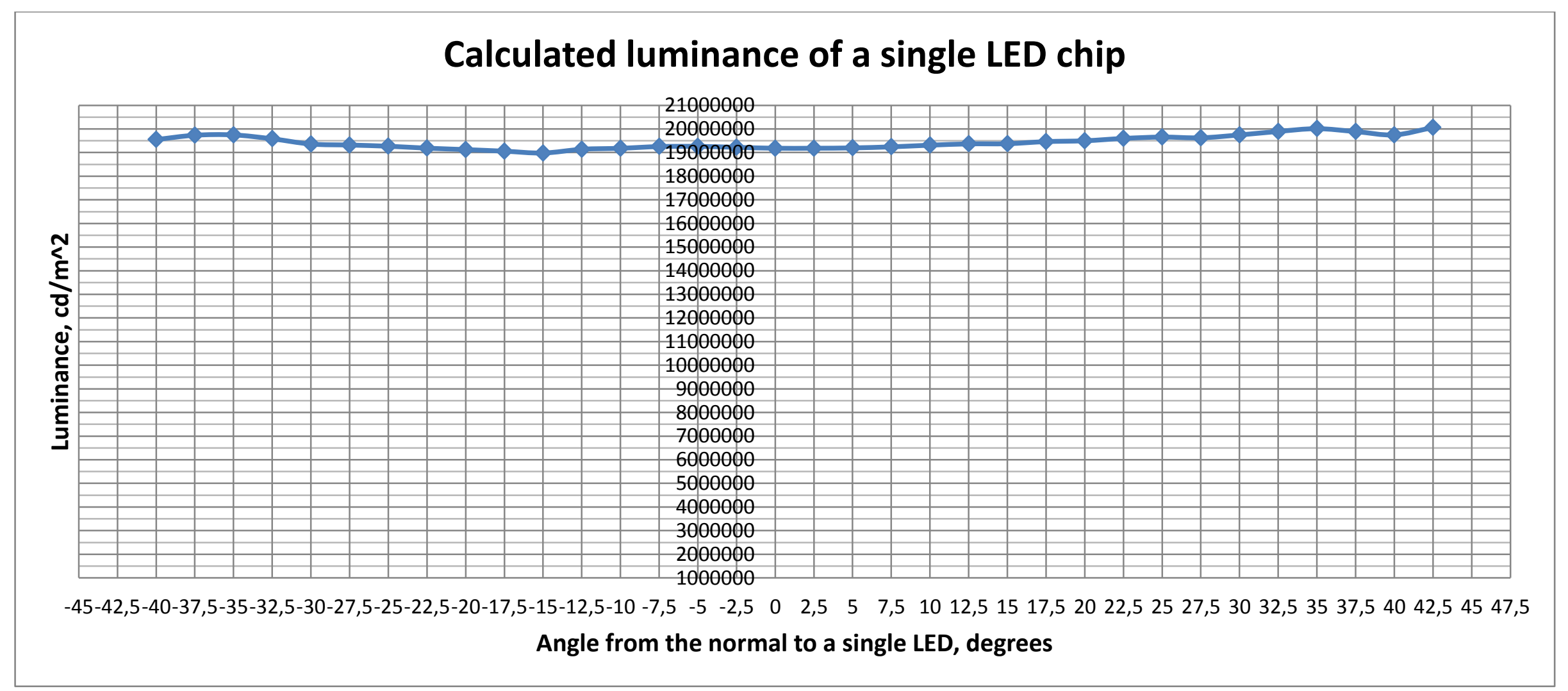

Figure 3-14. Calculated luminance values of a single LED chip depending on the angle, $\mathrm{cd} / \mathrm{m}^{2}$ 
Manufacturer's data on luminous intensity (appendix B) and measured/calculated data were compared to check the correspondence between the values. The manufacturer's data is given in relative units in the form of a graph. For accurate comparison GetData software was used to obtain relative values (GetData Graph Digitizier software). Calculated luminous intensity values were translated into the relative units. Both sets of data were put onto one graph (figure 3-17).

Manufacturer's data on luminous intesity curve was scanned. Numbers were assigned to the specified axes (figure $3-15)$. Origin of coordinates correspond to $(0,0)$ and maximum values to $(1,1)$.

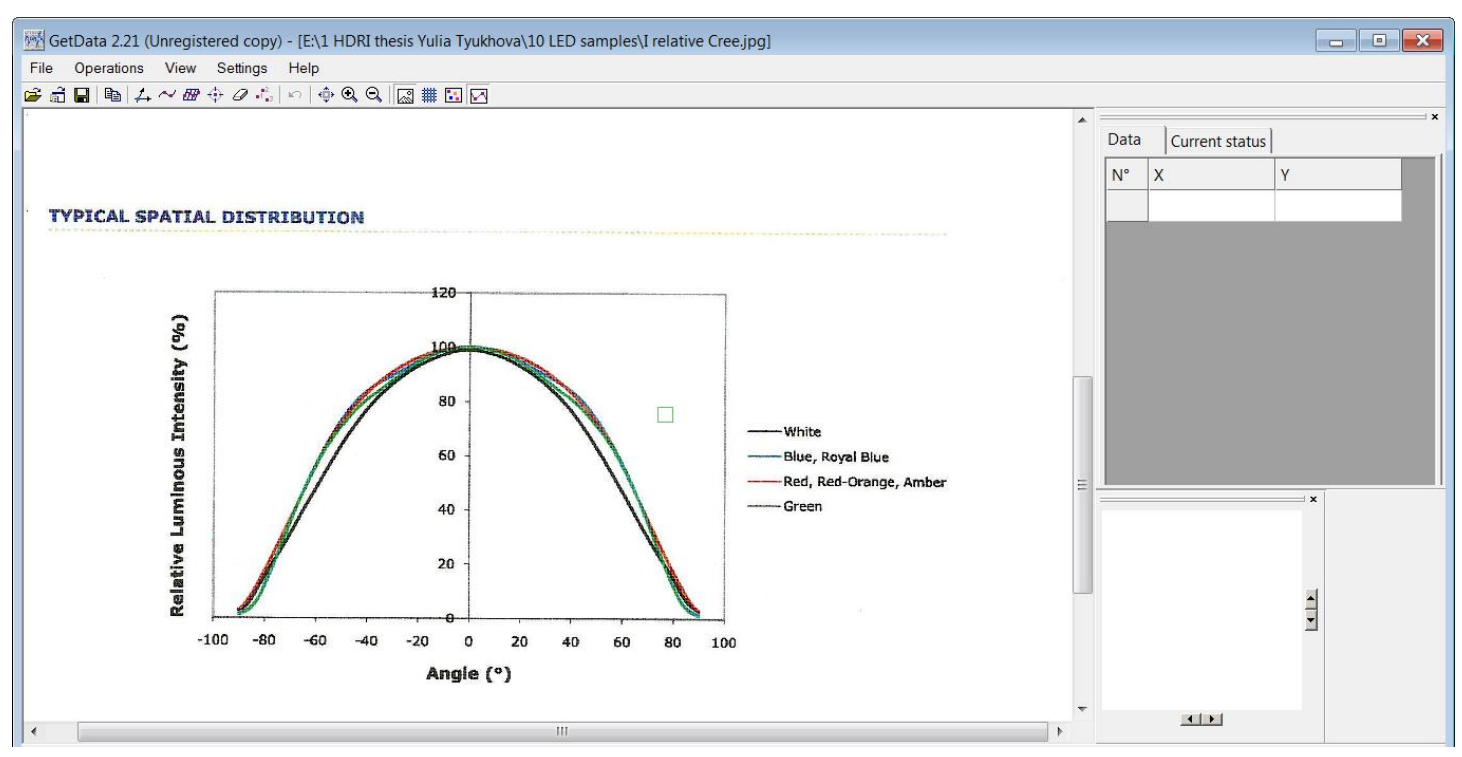

Figure 3-15. Specification of the axes over the scanned manufacturer's data on luminous intensity curve (GetData Graph Digitizier software)

The next step was to indicate points on the given curve with the point capture mode (figure 3-16). The software will estimate values according to the previously set coordinate system. 


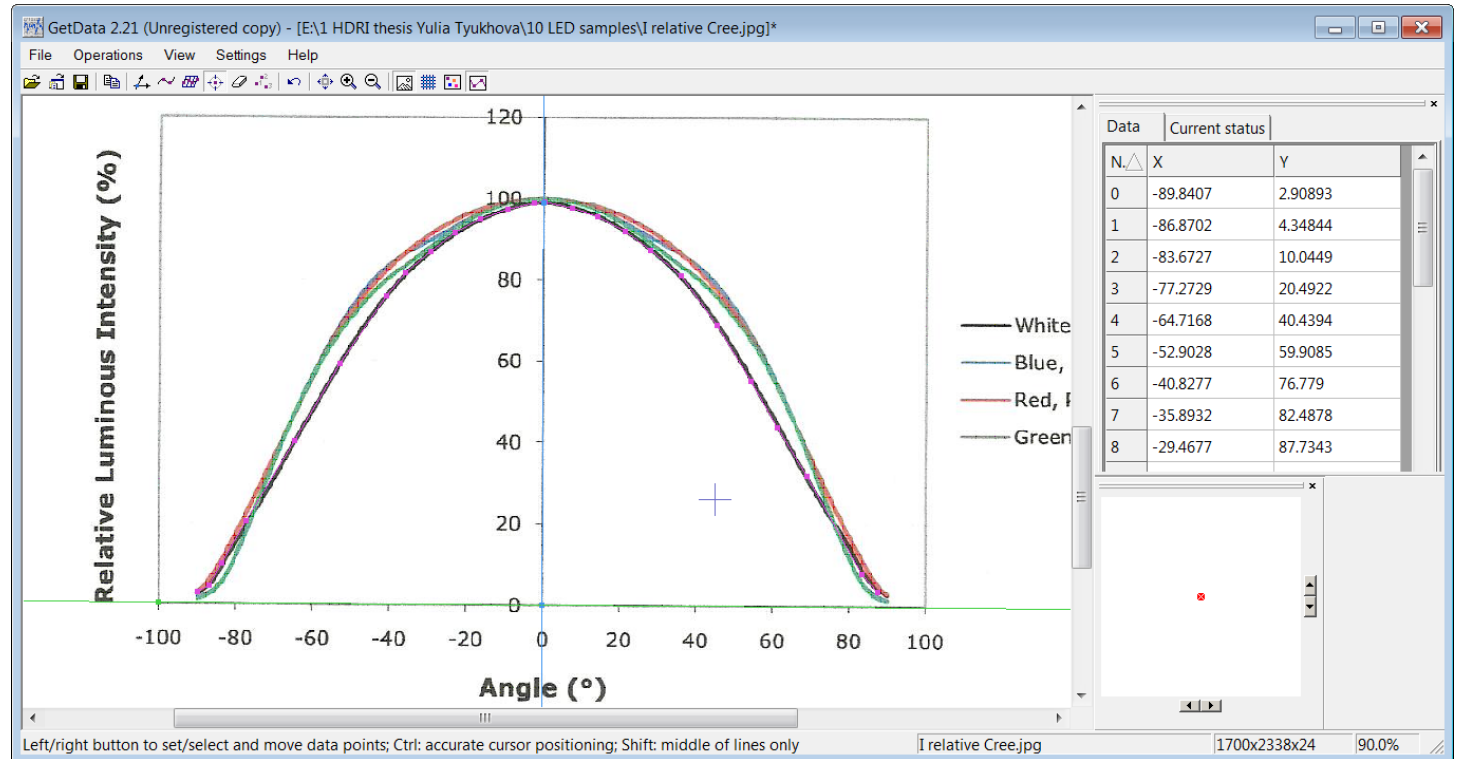

Figure 3-16. Obtaining data from the luminous intensity curve with the point capture mode (GetData Graph Digitizier software)

Then obtained points of manufacturer's data were put on the graph in relative units along with the experimental data (figure 3-17). The graph gives an indication in a relative manor that the measured data is similar to the manufacturer's data. 


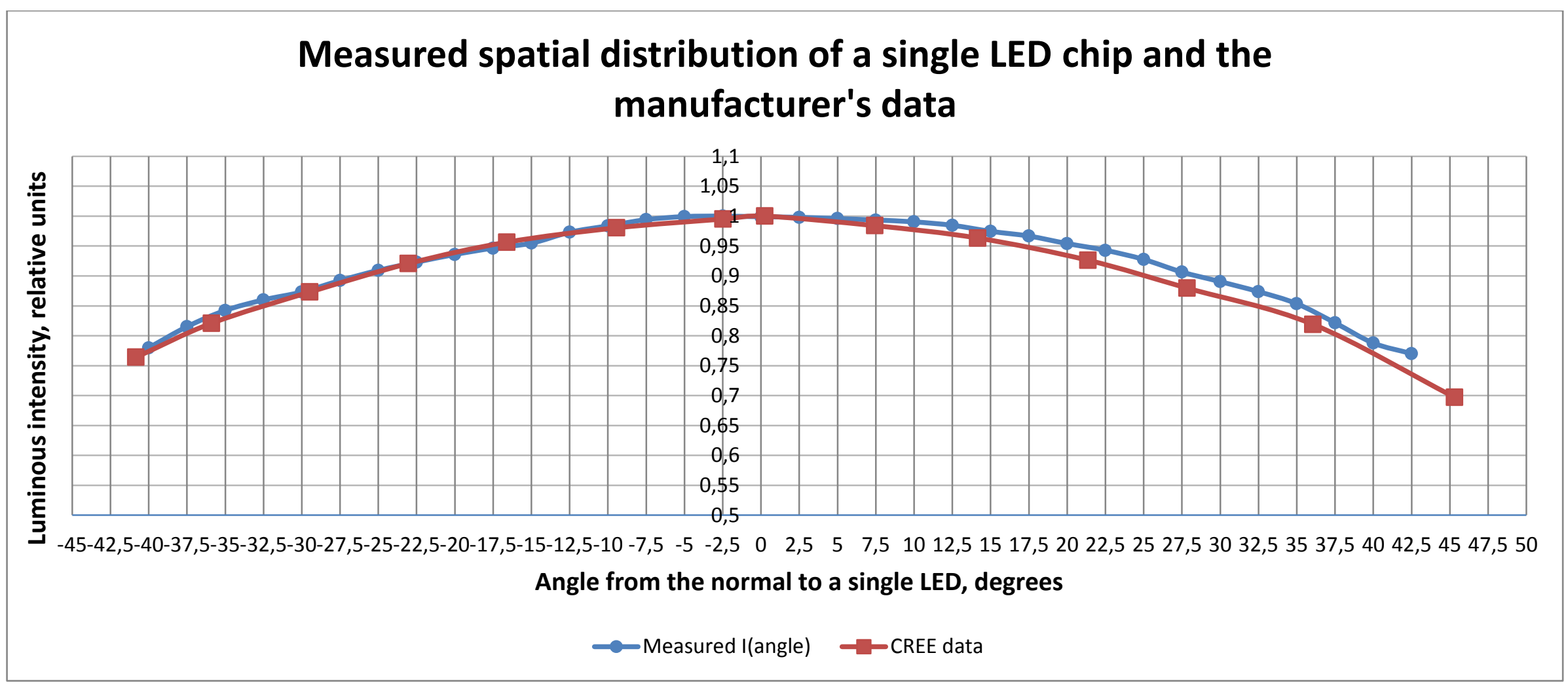

Figure 3-17. Digitalized manufacturer's and measured/calculated intensity curves of a single LED chip, relative units 
Outcome: With the assumption that the LED is a uniform light source the luminance value was obtained through direct illuminance measurements and subsequent calculations. The average luminance is $19.44 * 10^{6} \mathrm{~cd} / \mathrm{m}^{2}$.This value can be used in the comparison with the HDRI measurements.

\subsection{The implementation of the HDRI technique for lighting measurements}

This section discusses the implementation of the HDRI technique in lighting research. Two cameras and lenses were tested in various experiments to determine best technique to capture a single LED chip. Then HDRI acquisition of a single LED chip was performed (section 3.4).

\subsubsection{Response curves for cameras and lenses}

Goal: To derive the response curves for two cameras with two lenses.

The first step was to obtain camera response curves. The photographs were taken on the 2/10/12 sunny morning at 9:30 am in Omaha, NE. For deriving RCs interior office environment with windows was chosen. Office had neutral black and white colors with smooth gradients and very bright and dark areas. Settings of the experiments and measurements are provided in the tables 3-2-3-5.

Table 3-2. Settings for the response curve experiments

\begin{tabular}{|c|c|}
\hline Camera & CANON EOS Rebel T1i 500D \\
\hline Lens & CANON zoom lens EF28-105mm 1:3.5-4.5 II USM \\
\hline $\begin{array}{l}\text { Luminance } \\
\text { meter }\end{array}$ & Minolta LS110 \\
\hline $\begin{array}{l}\text { Reflectance } \\
\text { standard }\end{array}$ & $\begin{array}{c}\text { Labsphere (SRS-99-020, SRS-40-020,SRS-2-020), 99\%, 40\%, } \\
2 \%\end{array}$ \\
\hline Scene & $\begin{array}{l}\text { Interior daylighting, neutral colors, big smooth gradient, very } \\
\text { dark and very bright }\end{array}$ \\
\hline $\begin{array}{l}\text { Lighting } \\
\text { conditions }\end{array}$ & Clear sunny day, no clouds \\
\hline ISO & 100 \\
\hline WB & $6000 \mathrm{~K}$ \\
\hline Aperture size & $\mathrm{F} / 8.0$ \\
\hline Quality & Large \\
\hline Resolution & $15 \mathrm{M} 4752 \times 3168$ \\
\hline Metering mode & Evaluative \\
\hline E correction & Peripheral illumination correction is enabled \\
\hline
\end{tabular}




\begin{tabular}{|c|c|}
\hline $\begin{array}{c}\text { Sequence was } \\
\text { taken }\end{array}$ & Through the computer without touching the camera \\
\hline Focal length & $28 \mathrm{~mm}$ \\
\hline Focus & $2 \mathrm{~m}, 8^{\prime}$ \\
\hline Shutter speeds & $1 / 4000,1 / 2000,1 / 1000,1 / 500,1 / 250,1 / 125,1 / 60,1 / 30,1 / 15$, \\
& $1 / 8,1 / 4,1 / 2,11^{\prime}, 2^{\prime}, 4^{\prime}$, \\
\hline Mode & Manual \\
\hline
\end{tabular}

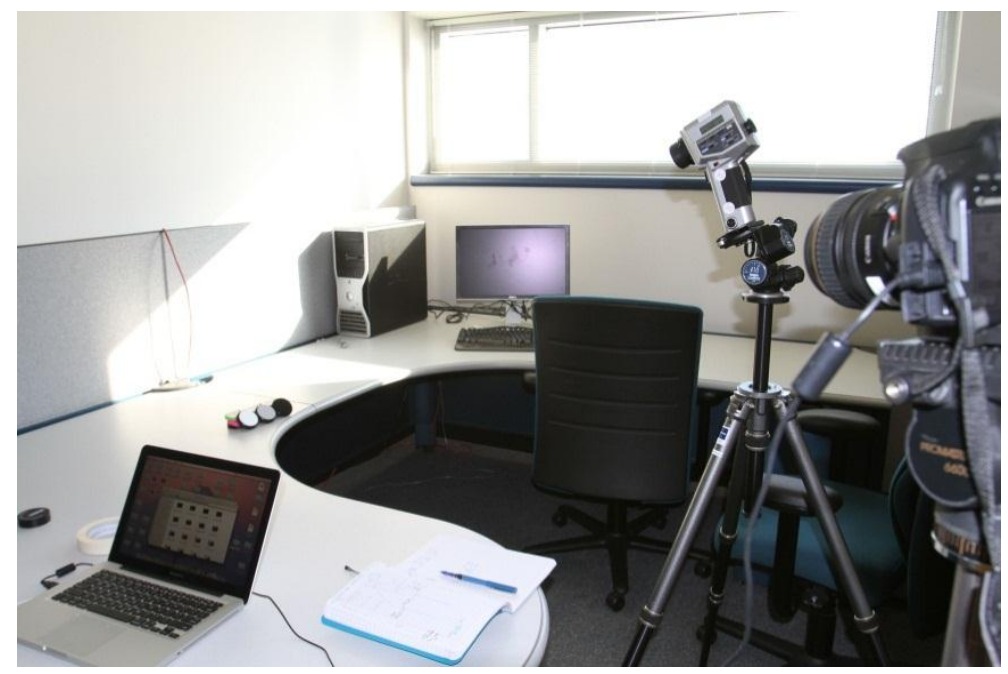

Figure 3-18. Experimental set-up for deriving the response curve

Luminance meter and the camera were set on the tripods (figure 3-18). Photos were taken through computer with CANON EOS Utility Software. Measurements of reflectance standards' luminance values (with the luminance meter LS110) were taken before and after the image sequence (figure 3-19). Then obtained measurements were averaged (see tables 3-3 and 3-5). Image sequence took no more than 1.5 minutes per camera.

Table 3-3. Luminance meter measurements of the reflectance standards

\begin{tabular}{|c|c|c|c|}
\hline $\begin{array}{c}\text { Reflectance } \\
\text { standard } \\
\text { measurements with } \\
\text { the luminance } \\
\text { meter, cd/m }\end{array}$ & White 99\% & Grey 40\% & Black 2\% \\
\hline $\begin{array}{c}\text { Before the sequence } \\
\text { of photos }\end{array}$ & 408.3 & 188 & 17.57 \\
\hline $\begin{array}{c}\text { After the sequence } \\
\text { of photos }\end{array}$ & 398 & 184.9 & 16.72 \\
\hline Average & 403.15 & 186.45 & 17.145 \\
\hline
\end{tabular}




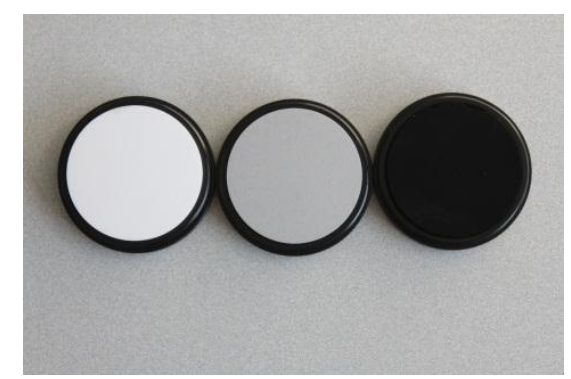

Figure 3-19. Reflectance standards $(99 \%, 40 \%, 2 \%)$

Photographs were fused in Photosphere. Measured RC coefficients can be obtained from Go >>Library >>Preferences $>>$ Photosphere (Mac OS).

$$
\operatorname{red}(r)=-6.439693 e-03+4.521897 \mathrm{e}-01 * \mathrm{r}+-1.292528 \mathrm{e}+00 * \mathrm{r}^{\wedge} 2+
$$

$1.804436 \mathrm{e}+00 * \mathrm{r}^{\wedge} 3$

$$
\operatorname{green}(\mathrm{g})=--5.809676 \mathrm{e}-03+4.179402 \mathrm{e}-01 * \mathrm{~g}+-1.177586 \mathrm{e}+00 * \mathrm{~g} \wedge 2+
$$

\section{$1.723113 \mathrm{e}+00 * \mathrm{~g}^{\wedge} 3$}

blue $(b)=-4.380568 \mathrm{e}-03+3.787649 \mathrm{e}-01 * \mathrm{~b}+-1.076614 \mathrm{e}+00 * \mathrm{~b}^{\wedge} 2+$

\section{$1.659887 \mathrm{e}+00 * \mathrm{~b}^{\wedge} 3$.}

Then hand-held calibration was made at the grey standard $\left(186.45 \mathrm{~cd} / \mathrm{m}^{2}\right)$ with the luminance meter. Calibration coefficient $(\mathrm{CF})$ is 0.96 .

See tables 3-4 and 3-5 for the second camera and lens settings and measurements.

\begin{tabular}{|c|c|}
\hline Camera & CANON EOS 7D \\
\hline Lens & Canon EF 16-35mm f/2.8L II USM \\
\hline $\begin{array}{l}\text { Luminance } \\
\text { meter }\end{array}$ & Minolta LS110 \\
\hline $\begin{array}{l}\text { Reflectance } \\
\text { standard }\end{array}$ & $\begin{array}{c}\text { Labsphere (SRS-99-020, SRS-40-020,SRS-2-020), 99\%, 40\%, } \\
2 \%\end{array}$ \\
\hline Scene & $\begin{array}{c}\text { Interior daylighting, neutral colors, big smooth gradient, very } \\
\text { dark and very bright }\end{array}$ \\
\hline $\begin{array}{l}\text { Lighting } \\
\text { conditions }\end{array}$ & Clear sunny day, no clouds \\
\hline ISO & 100 \\
\hline WB & Custom $6500 \mathrm{~K}$ \\
\hline Aperture size & $\mathrm{F} / 8.0$ \\
\hline Quality & Large + RAW \\
\hline
\end{tabular}

Table 3-4. Settings for the response curve experiments 


\begin{tabular}{|c|c|}
\hline Resolution & 17.9 M 5184x3456 \\
\hline Metering mode & Evaluative \\
\hline E correction & Peripheral illumination correction is enabled \\
\hline $\begin{array}{c}\text { Sequence was } \\
\text { taken }\end{array}$ & Through the computer without touching the camera \\
\hline Focal length & $16 \mathrm{~mm}$ \\
\hline Focus & $2 \mathrm{~m}, 8^{\prime}$ \\
\hline Shutter speeds & $1 / 8000,1 / 4000,1 / 2000,1 / 1000,1 / 500,1 / 250,1 / 125,1 / 60,1 / 30$, \\
& $1 / 15,1 / 8,1 / 4,1 / 2,1,2,2^{\prime},{ }^{\prime}$, \\
\hline Mode & Manual \\
\hline
\end{tabular}

Table 3-5. Luminance meter measurements of the reflectance standards

\begin{tabular}{|c|c|c|c|}
\hline $\begin{array}{c}\text { Reflectance } \\
\text { standard } \\
\text { measurements with } \\
\text { the luminance } \\
\text { meter, cd/m }\end{array}$ & White 99\% & Grey 40\% & Black 2\% \\
\hline $\begin{array}{c}\text { Before the sequence } \\
\text { of photos }\end{array}$ & 421.8 & 192.8 & 18.75 \\
\hline $\begin{array}{c}\text { After the sequence } \\
\text { of photos }\end{array}$ & 413.2 & 190.4 & 18.2 \\
\hline Average & 417.5 & 191.6 & 184.8 \\
\hline
\end{tabular}

The sequence of images for the second camera and lens was calibrated at the grey card $\left(191.6 \mathrm{~cd} / \mathrm{m}^{2}\right) . \quad C F=1.038$.

RC coefficients:

$\operatorname{red}(\mathrm{r})=-4.884809 \mathrm{e}-03+4.246084 \mathrm{e}-01 * \mathrm{r}+-1.162948 \mathrm{e}+00 * \mathrm{r}^{\wedge} 2+$

$1.781711 \mathrm{e}+00 * \mathrm{r}^{\wedge} 3$

$\operatorname{green}(\mathrm{g})=-4.765016 \mathrm{e}-03+4.014215 \mathrm{e}-01 * \mathrm{~g}+-1.095227 \mathrm{e}+00 * \mathrm{~g}^{\wedge} 2+$

$1.737057 \mathrm{e}+00 * \mathrm{~g} \wedge 3$

blue $(b)=-2.361507 \mathrm{e}-03+3.261316 \mathrm{e}-01 * \mathrm{~b}+-8.961151 \mathrm{e}-01 * \mathrm{~b}^{\wedge} 2+$

$1.610831 \mathrm{e}+00 * \mathrm{~b}^{\wedge} 3$.

Outcome: After obtaining response curves and making hand-held calibrations with the luminance meter, cameras can be used as luminance mapping devices.

\subsubsection{Optical vignetting}

Goal: To check optical vignetting effect under various conditions. 


\subsubsection{Description of experimental setting}

Vignetting effect can be assessed by making an HDRI of a uniformly lit surface. For this research vignetting effect was assessed by making an HDR image of the uniform luminance source.

Experimental setting for vignetting experiments is shown on figure 3-20. Uniform light source was set to have a certain level of luminance. Camera was set next to the sphere on a tripod, and the sequence of photos was taken through the computer with no ambient light on.

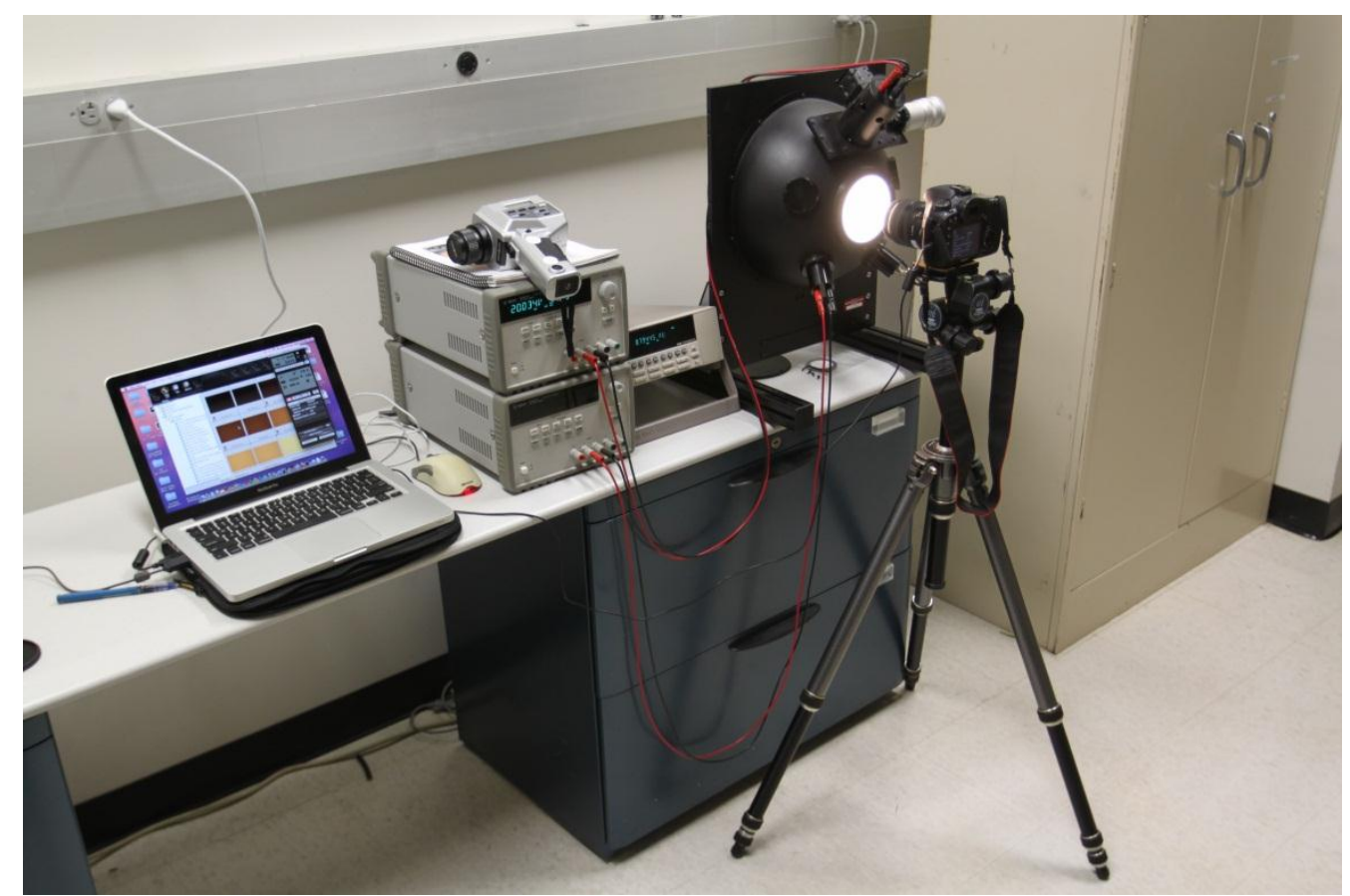

Figure 3-20. Experimental set-up for evaluating optical vignetting effect

The description of ULS can be found in Appendix B.

\subsubsection{Checking uniformity of the ULS}

Even though ULS is a variation of an integrating sphere and should in theory be very uniform, an experiment checking this was conducted.

Ambient light was turned off. Incandescent lamp had to be turned on for about 45 minutes in the sphere to stabilize the light output. Then measurements of 
luminance were taken in five points with the calibrated LS110 luminance meter without any close-up lenses. Luminance meter was set on a tripod 1 meter away from the sphere.

Luminance readings were taken in five points (see figure 3-21 and table 3-6).

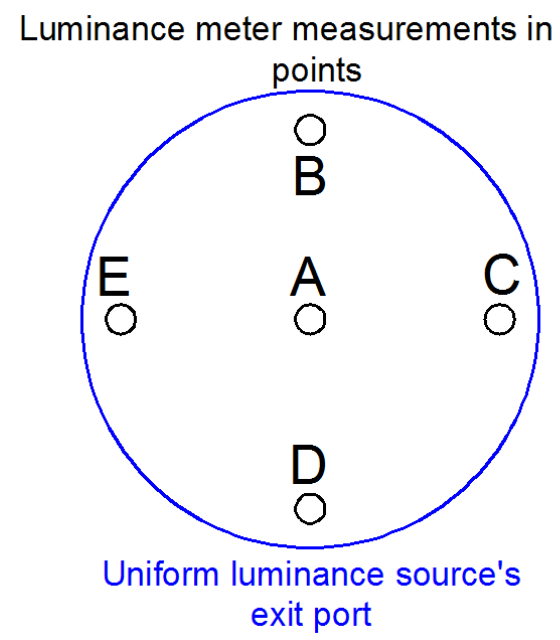

Figure 3-21. Five points of ULS of an exit port where luminance values are measured

Table 3-6. Luminance readings of uniform luminance source in five points of an exit port

\begin{tabular}{|c|c|c|}
\hline $\begin{array}{c}\text { Measuring point of the } \\
\text { ULS exit port }\end{array}$ & $\begin{array}{c}\mathbf{L}, \mathbf{c d} / \mathbf{m}^{\mathbf{2}} \text { (before } \\
\text { vignetting experiments) }\end{array}$ & $\begin{array}{c}\mathbf{L}, \mathbf{c d} / \mathbf{m}^{\mathbf{2}} \text { (between } \\
\text { vignetting } \\
\text { experiments) }\end{array}$ \\
\hline A & 8418 & 8413 \\
\hline B & 8404 & 8405 \\
\hline C & 8427 & 8426 \\
\hline D & 8439 & 8440 \\
\hline E & 8410 & 8424 \\
\hline
\end{tabular}

To calculate the error of non-uniformity the following formula was used:

$$
\text { Error }=\frac{\left|L_{1}-L_{2}\right|}{L_{1}} \times 100 \%
$$

Outcome: The non-uniformity of ULS is less than $1 \%$. ULS can be considered perfectly uniform field to test the optical vignetting effect of the camera/lens combination. 


\subsubsection{Build-in peripheral illumination correction experiment}

Interesting aspect to investigate is the built-in peripheral illumination correction that should compensate vignetting effect within the camera (according to Canon website as of January 2011). CANON manual reports that camera has a profile for 25 lenses in the memory and more can be uploaded from the website.

For the purpose of this experiment two HDRIs of ULS were obtained with the EOS7D CANON camera and CANON 16-35 mm zoom lens with peripheral illumination correction on and off (table 3-7). Other camera settings were set as recommended in section 2.2.1.2.

Table 3-7. Camera settings to test the built-in peripheral illumination correction

\begin{tabular}{|c|c|}
\hline Camera & CANON EOS 7D \\
\hline Lens & Canon EF 16-35mm f/2.8L II USM \\
\hline Goal & $\begin{array}{c}\text { Test the built-in peripheral illumination correction results for } \\
\text { EOS7D and the lens 16-35mm }\end{array}$ \\
\hline WB & $6500 \mathrm{~K}$ \\
\hline Focal length & $16 \mathrm{~mm}$ \\
\hline Aperture size & F/8.0 \\
\hline Focus & Manual, 0.28m \\
\hline $\begin{array}{c}\text { Peripheral } \\
\text { Illumination } \\
\text { correction }\end{array}$ & On/off \\
\hline Shutter speed & \\
\hline
\end{tabular}

Picoammeter readings were made before and after the photo sequence was taken to check the stability of the incandescent lamp voltage, so the light level was constant. It was found that voltage was stable during all the experiments $(0.01 \%$ error).

The HDRI was obtained by fusing LDR photos with the known response curve for the camera and lens combination in Photosphere. Photosphere provides some statistical data of the selected area (mean, median, minimum, maximum values and standard deviation in logarithm domain (see appendix E)) along with the histogram for luminance and the RGB values. By highlighting certain part of the image in 
Photosphere, histogram is updated automatically according to the information within the selected rectangle.

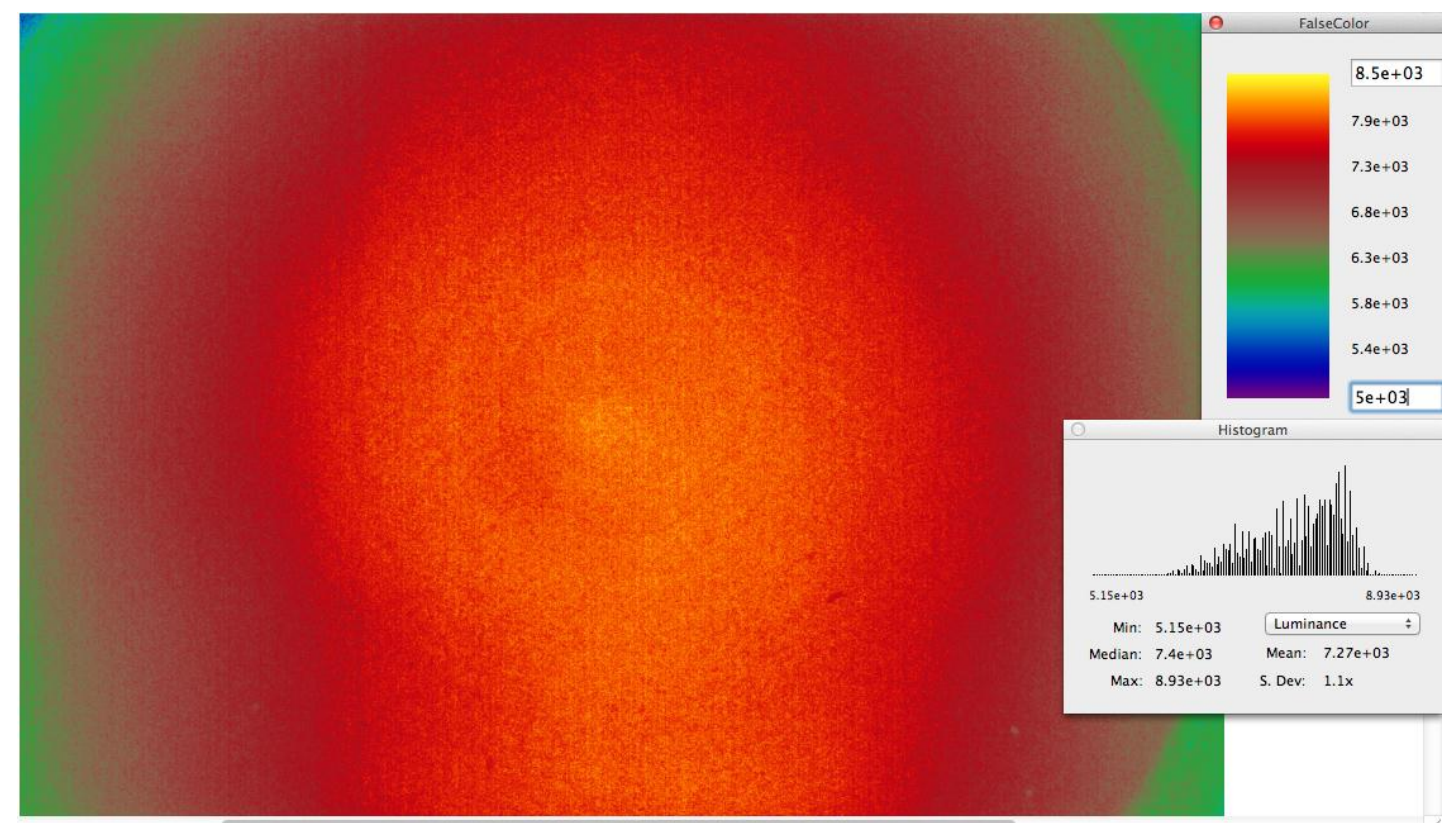

Figure 3-22. HDRI of ULS with peripheral Illumination correction on (CANON EOS 7D with zoom lens 16-35 mm)

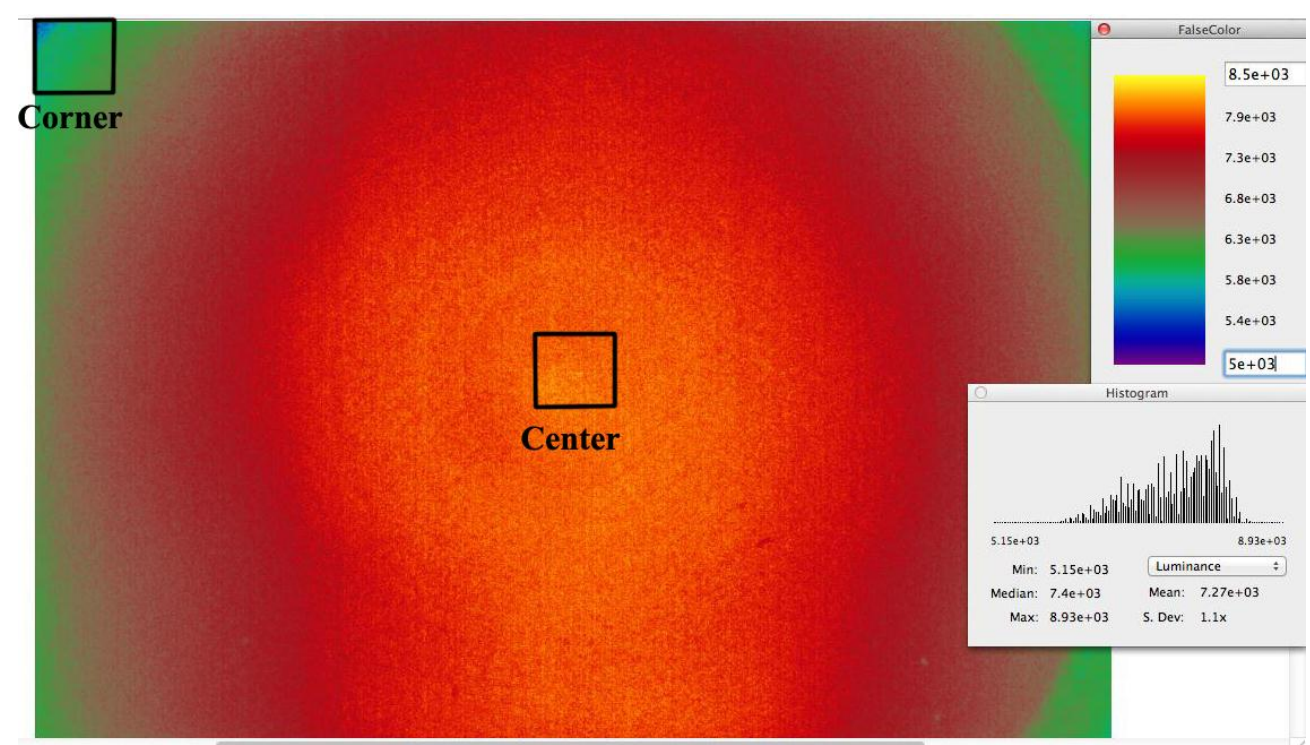

Figure 3-23. HDRI of ULS and the analyzed areas

To estimate the error due to the optical vignetting effect in the image, mean values for corner and center selected areas were obtained in Photosphere (figure 323). The highlighted area is about 350 pixels x 350 pixels.

Mean value of the center is $\mathrm{L}_{1}=7950 \mathrm{~cd} / \mathrm{m}^{2}$. 
Mean value of the corner is $\mathrm{L}_{2}=5990 \mathrm{~cd} / \mathrm{m}^{2}$.

Error $1=\frac{\left|L_{1}-L_{2}\right|}{L_{1}} \times 100 \%=25 \%$ light fall off (figure 3-22).

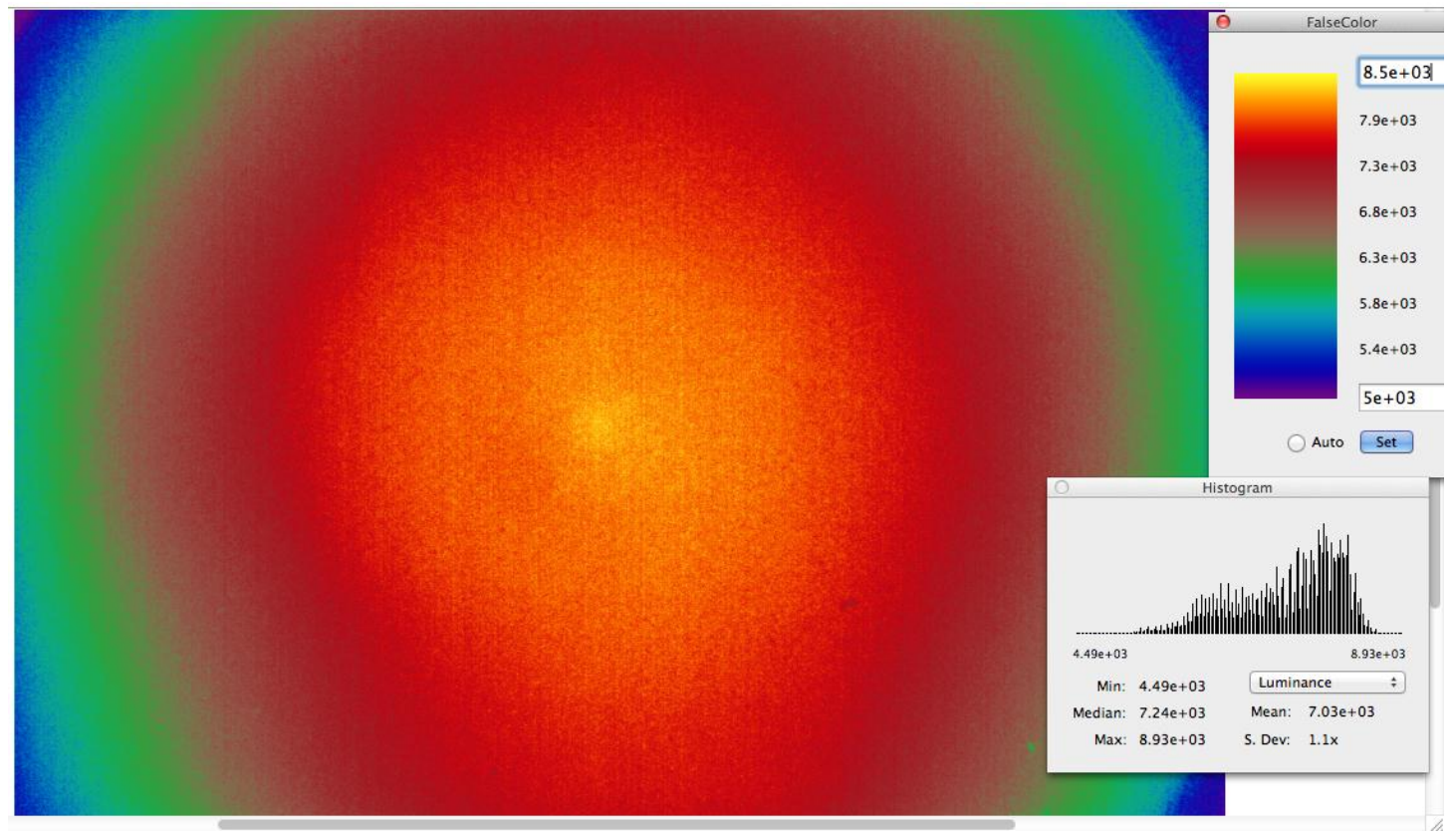

Figure 3-24. HDRI of ULS with peripheral Illumination correction off (CANON EOS 7D with zoom lens 16-35 mm)

Mean value of the corner is $5320 \mathrm{~cd} / \mathrm{m}^{2}$.

Mean value of the center is $8050 \mathrm{~cd} / \mathrm{m}^{2}$.

Error $2=\frac{\left|L_{1}-L_{2}\right|}{L_{1}} \times 100 \%=34 \%$ light fall off (figure 3-24).

For the convenient comparison both HDRIs were scaled to the same pseudo colors range $\left(8500-5000 \mathrm{~cd} / \mathrm{m}^{2}\right)$. By comparing the two images it can be clearly seen that peripheral illumination correction does improve the quality of HDRI.

The difference between the errors in luminance values due to the optical vignetting effect for two obtained images with peripheral illumination correction on and off is about $9 \%$.

Built-in peripheral illumination correction does improve the quality of HDRI, but not as it is necessary for photometric measurements. It doesn't compensate light fall off within the acceptable error. 
Initially camera is not developed for luminance measurements, so

manufacturer wouldn't account for the accurate photometric measurements. Visually one can't see light fall off towards the edges on the photograph.

Outcome: Image sequence used for creating HDRI should be taken with enabled built-in peripheral illumination correction in the camera, but this capability is not enough to compensate for vignetting effect during photometric measurements.

\subsubsection{Optical vignetting effect with different zoom settings}

Optical vignetting effect was checked for CANON EOS7D camera with the lens $16-35 \mathrm{~mm}$ lens at different zoom settings (table 3-8).

Table 3-8. Camera settings to test the vignetting effect for one camera/lens combination with different zoom settings

\begin{tabular}{|c|c|}
\hline Camera & CANON EOS7D \\
\hline Lens & Canon EF 16-35mm f/2.8L II USM \\
\hline Goal & Check the vignetting effect at different zooms \\
\hline WB & 6500K \\
\hline Focal length & Manual, 0.28m \\
\hline Focus & $1 / 8000-4$ '' (separated by 1 EV) \\
\hline Shutter speed & On \\
\hline $\begin{array}{c}\text { Peripheral } \\
\text { illumination } \\
\text { correction }\end{array}$ & \\
\hline
\end{tabular}

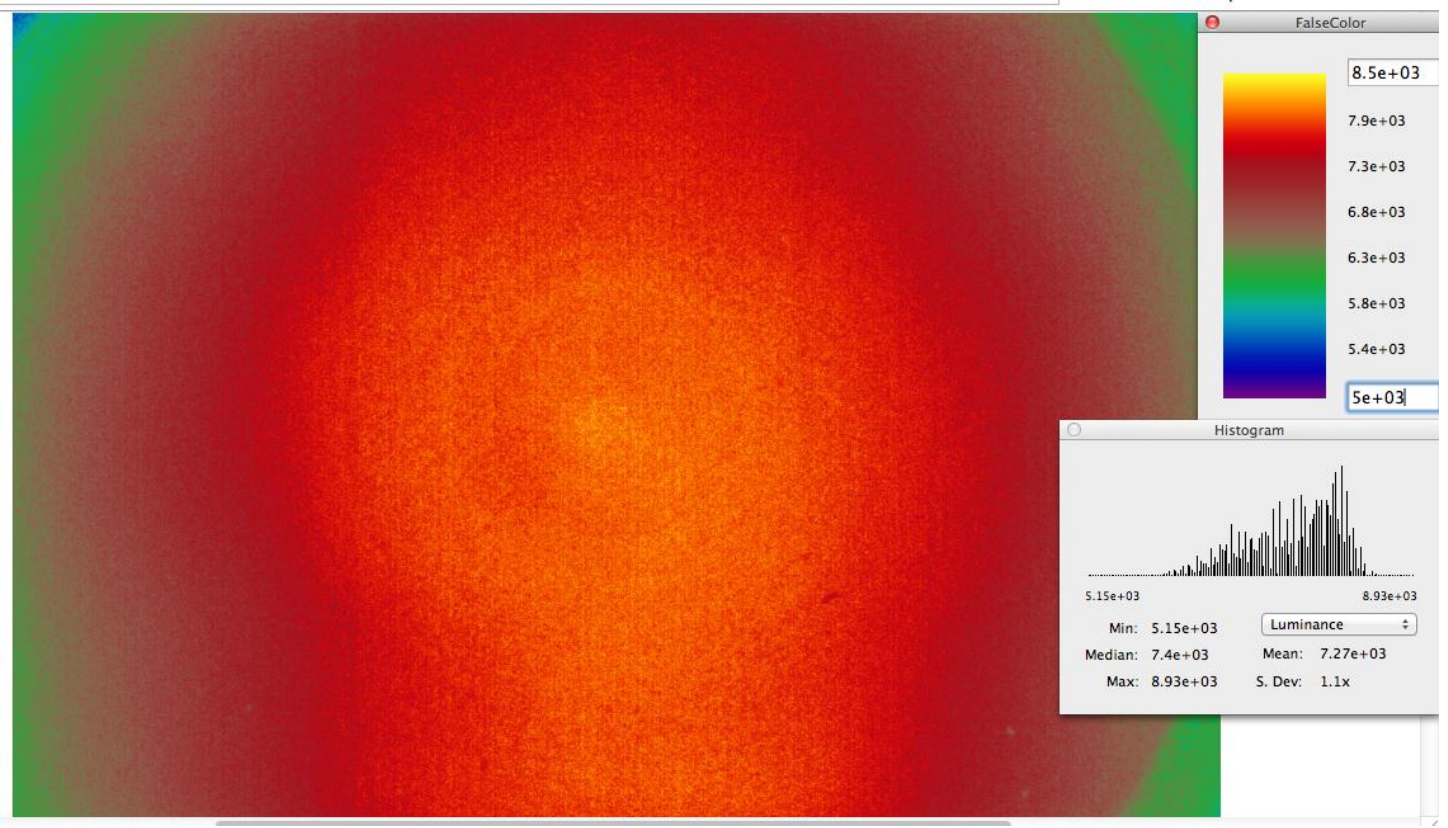

Figure 3-25. HDRI of ULS at $16 \mathrm{~mm}$ focal length 
Analyzed areas are the same as in previous section (center and corner 350 pixels by 350 pixels).

Mean value of the corner is $5990 \mathrm{~cd} / \mathrm{m}^{2}$.

Mean value of the center is $7950 \mathrm{~cd} / \mathrm{m}^{2}$.

Error $1=\frac{\left|L_{1}-L_{2}\right|}{L_{1}} \times 100 \%=25 \%$ light fall off.

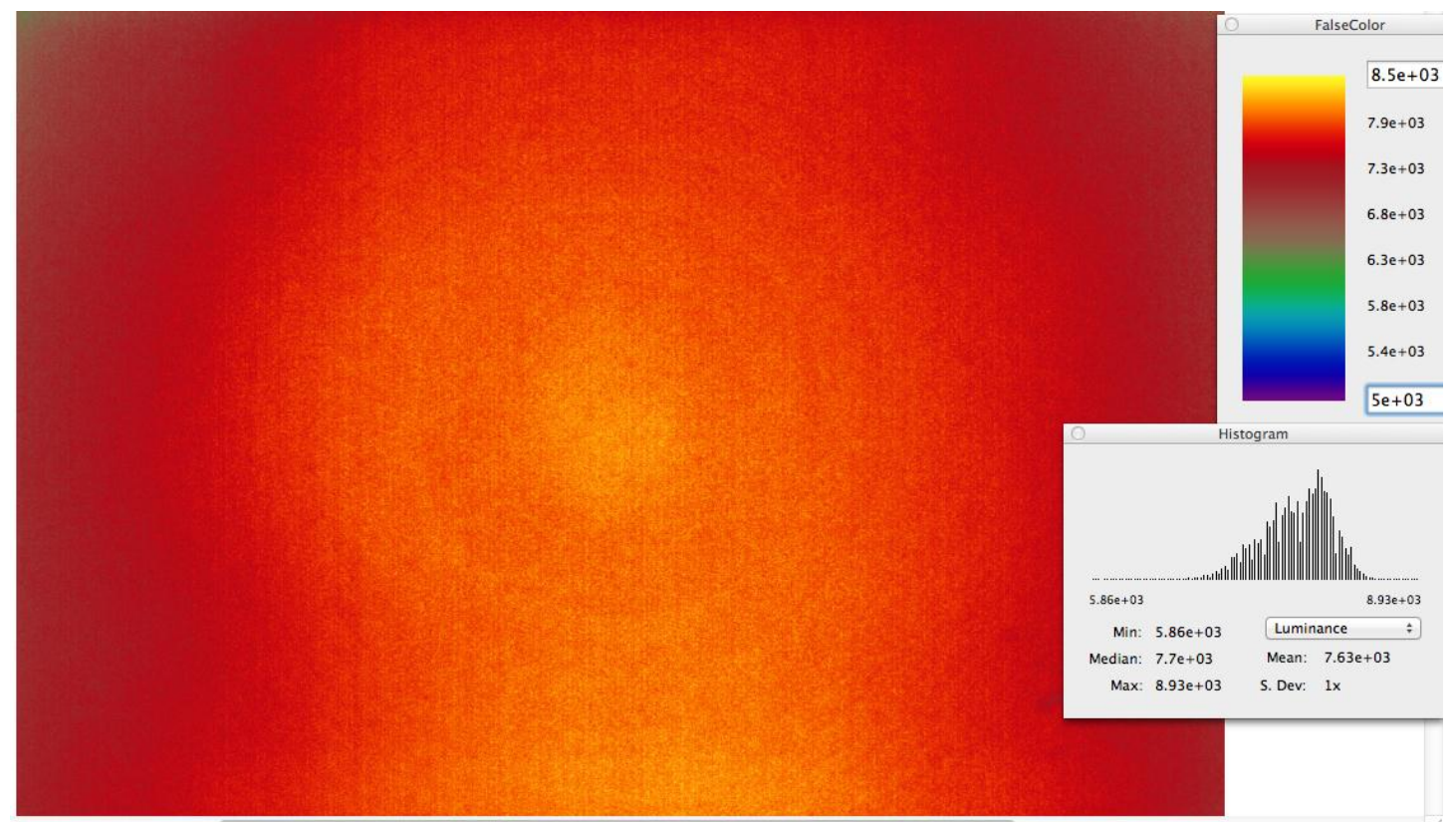

Figure 3-26. HDRI of ULS at $26 \mathrm{~mm}$ focal length

Mean value of the corner is $6720 \mathrm{~cd} / \mathrm{m}^{2}$.

Mean value of the center is $7980 \mathrm{~cd} / \mathrm{m}^{2}$.

Error $2=\frac{\left|L_{1}-L_{2}\right|}{L_{1}} \times 100 \%=16 \%$ light fall off. 


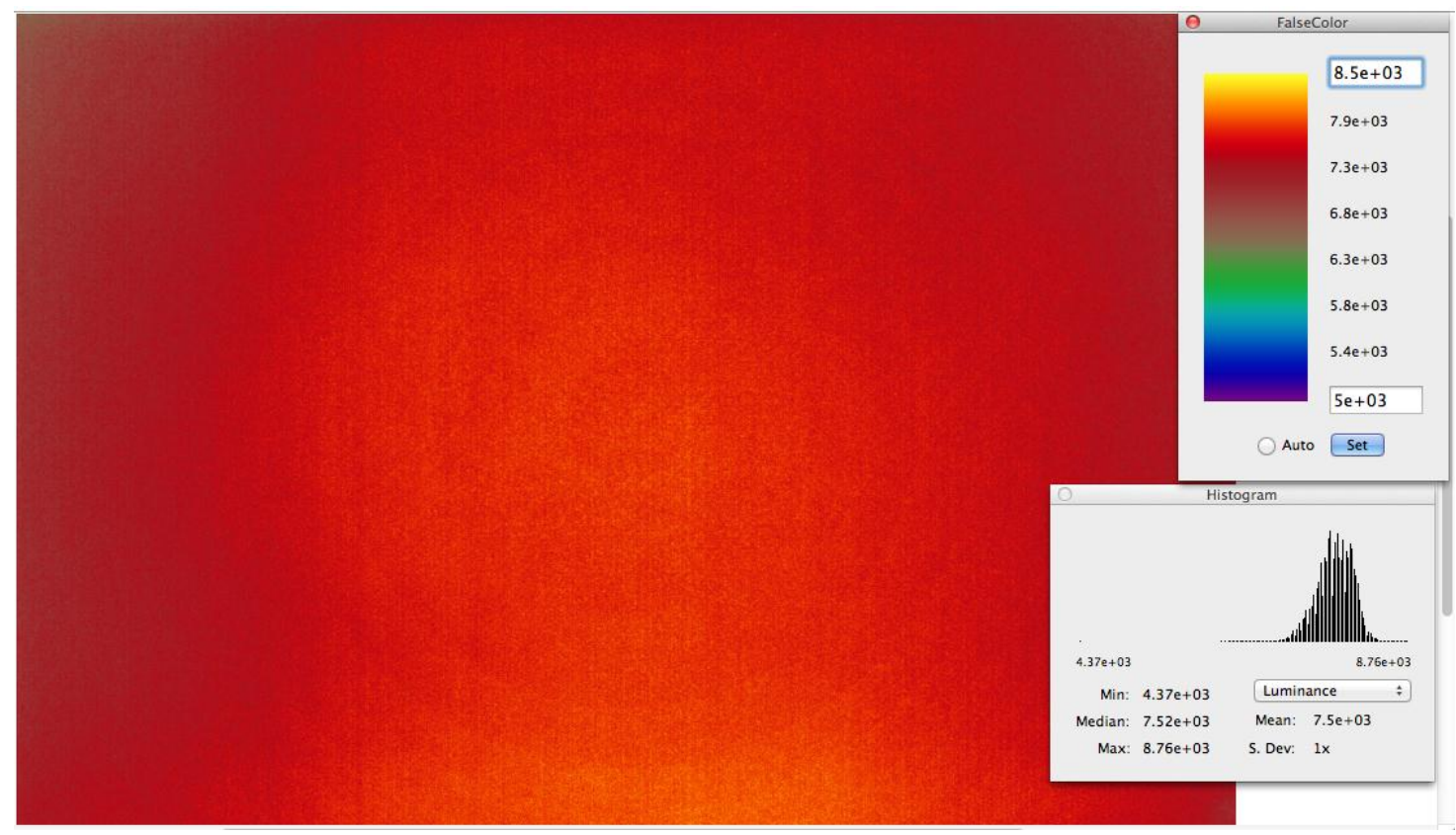

Figure 3-27. HDRI of ULS at $35 \mathrm{~mm}$ focal length

Mean value of the corner is $6760 \mathrm{~cd} / \mathrm{m}^{2}$.

Mean value of the center is $7710 \mathrm{~cd} / \mathrm{m}^{2}$.

Error $3=\frac{\left|L_{1}-L_{2}\right|}{L_{1}} \times 100 \%=12 \%$ light fall off.

All three images were scaled to the same pseudo color ranges (5000-8500 $\left.\mathrm{cd} / \mathrm{m}^{2}\right)$.

Outcome: For EOS7D fitted with CANON zoom lens 16-35mm vignetting effect is reduced when zoomed in, which is consistent with some publications (Moeck et al. 2006).

Vignetting effect depending on different variables should be thoroughly tested to accumulate more statistical data. This can be a separate study.

\subsubsection{Defining the experimental settings for capturing an HDRI of a single LED chip in GE garage fixture}

This section investigates experimental settings for capturing a single LED. 


\subsubsection{Capture of a single LED chip trial}

The luminaire was placed on a table. Only one LED chip was uncovered, all others were covered by non-transparent black fabric. Tripod was used for the camera EOS 7D fitted with CANON zoom lens16-35 mm (figure 3-28). Camera was aimed normal to the LED chip. The sequence of images of the LED was taken with the shutter speeds 1/8000-4', and the aperture size f/8 (other settings were set as recommended in section 2.2.1.2). The RC for this combination of camera/lens was used to fuse images in Photosphere (figure 3-29).

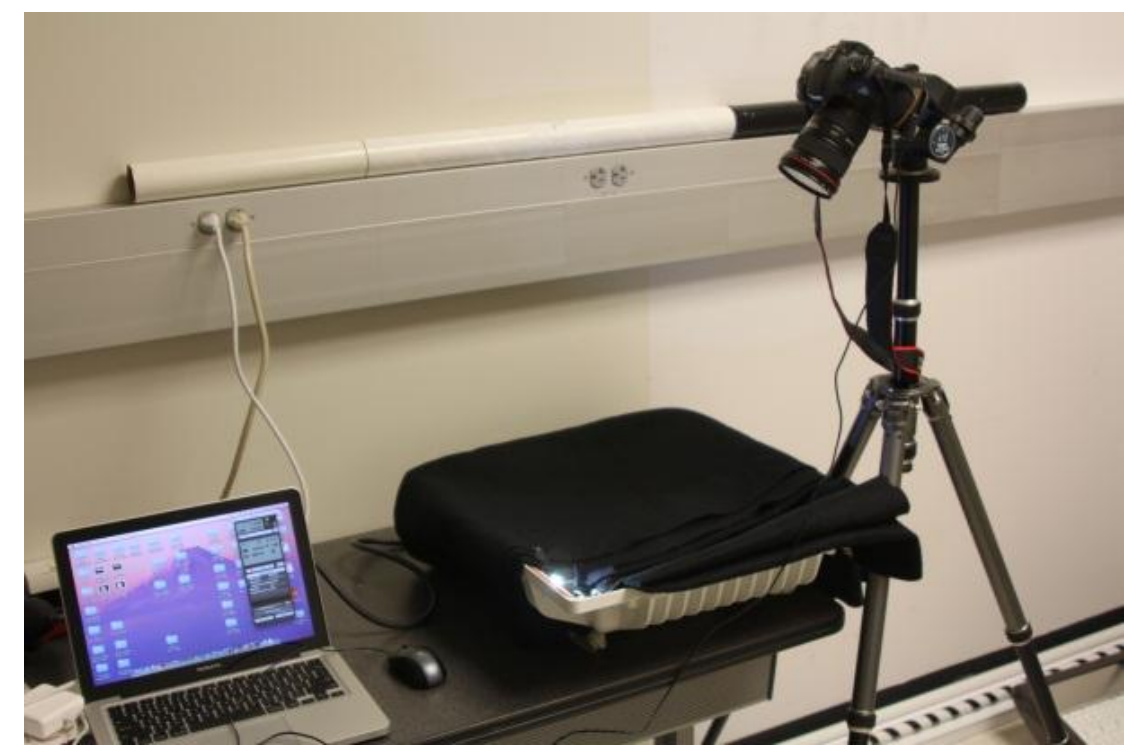

Figure 3-28. Experimental setting for obtaining an HDRI of the LED 


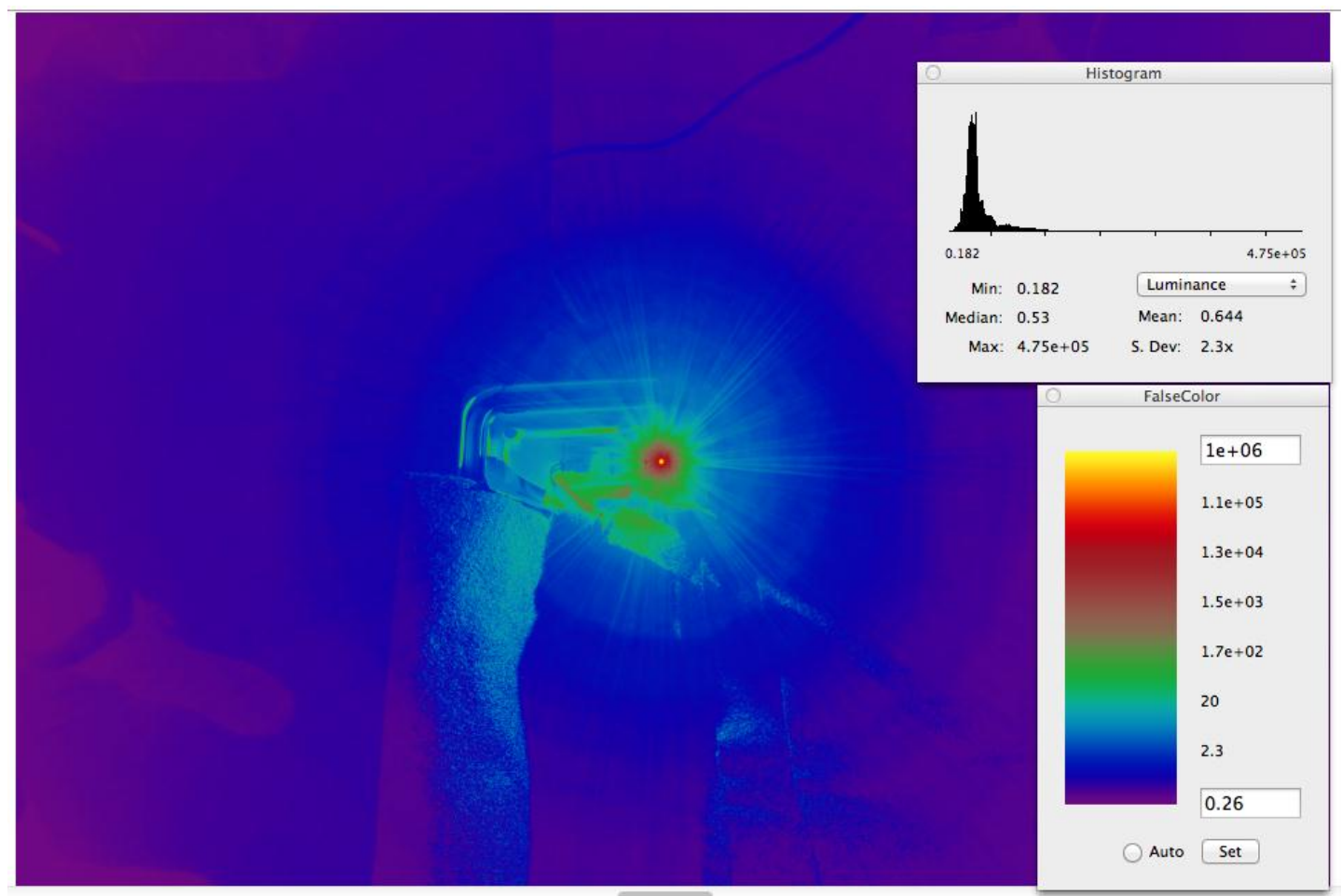

Figure 3-29. Pseudo colors HDRI of a single LED chip (obtained with CANON EOS 7D at $16 \mathrm{~mm}$, normal to the LED)

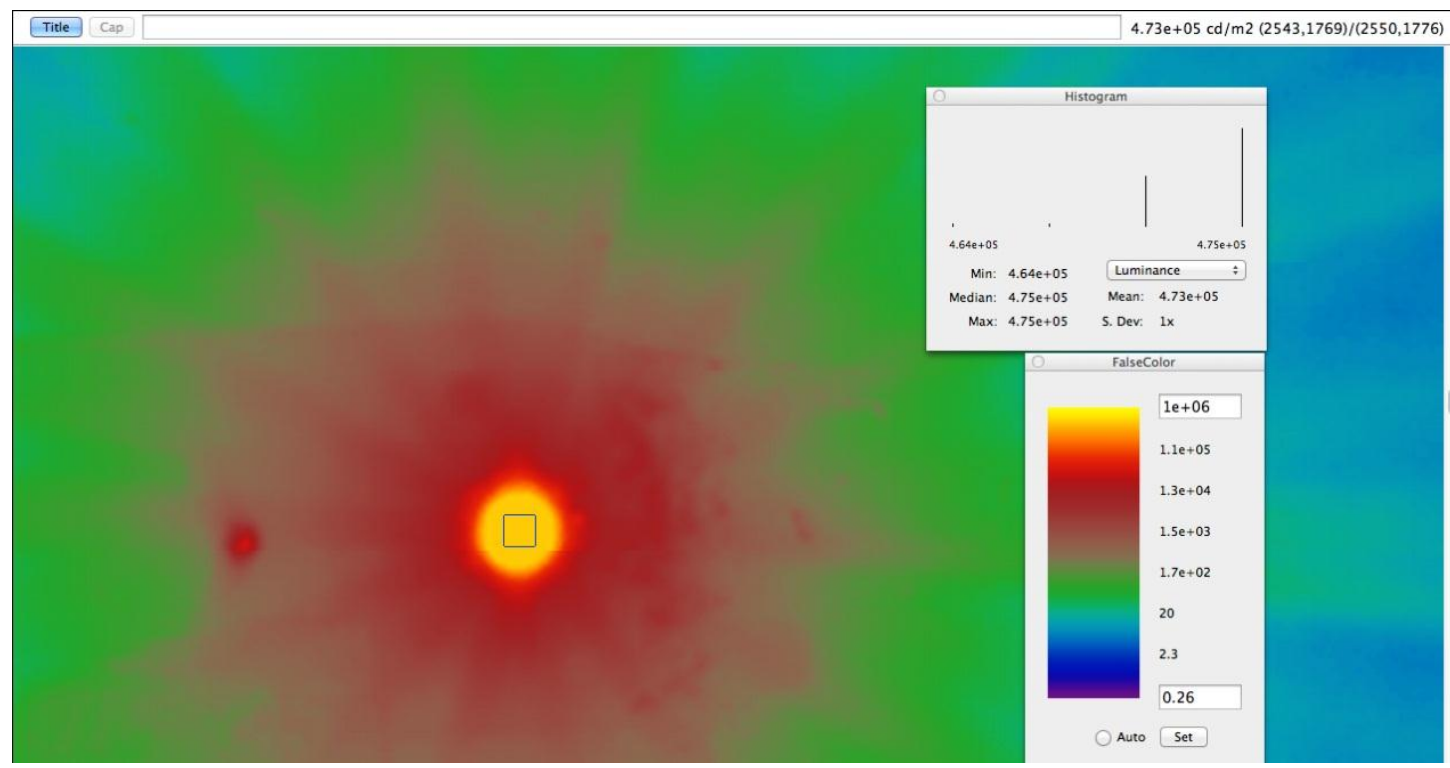

Figure 3-30. Statistical data for a single LED in Photosphere

Luminance values of a single LED chip are (figure 3-30):

Mean $=4.73 * 10^{5} \mathrm{~cd} / \mathrm{m}^{2}$

$\operatorname{Min}=4.64 * 10^{5} \mathrm{~cd} / \mathrm{m}^{2}$

$\operatorname{Max}=4.75 * 10^{5} \mathrm{~cd} / \mathrm{m}^{2}$ 
Median $=4.75 * 10^{5} \mathrm{~cd} / \mathrm{m}^{2}$

Outcome: According to sections 3.2.2, 3.2.3, where luminance is measured with traditional methods, obtained HDRI luminance is underestimated. Therefore, further analysis has to be conducted.

\subsubsection{Analysis of a scene used for obtaining the response curve}

When RC for a specific combination of camera/lens is obtained, the maximum value within the calibrating scene is $1.01 * 10^{5} \mathrm{~cd} / \mathrm{m}^{2}$ (figure $3-31$ ).

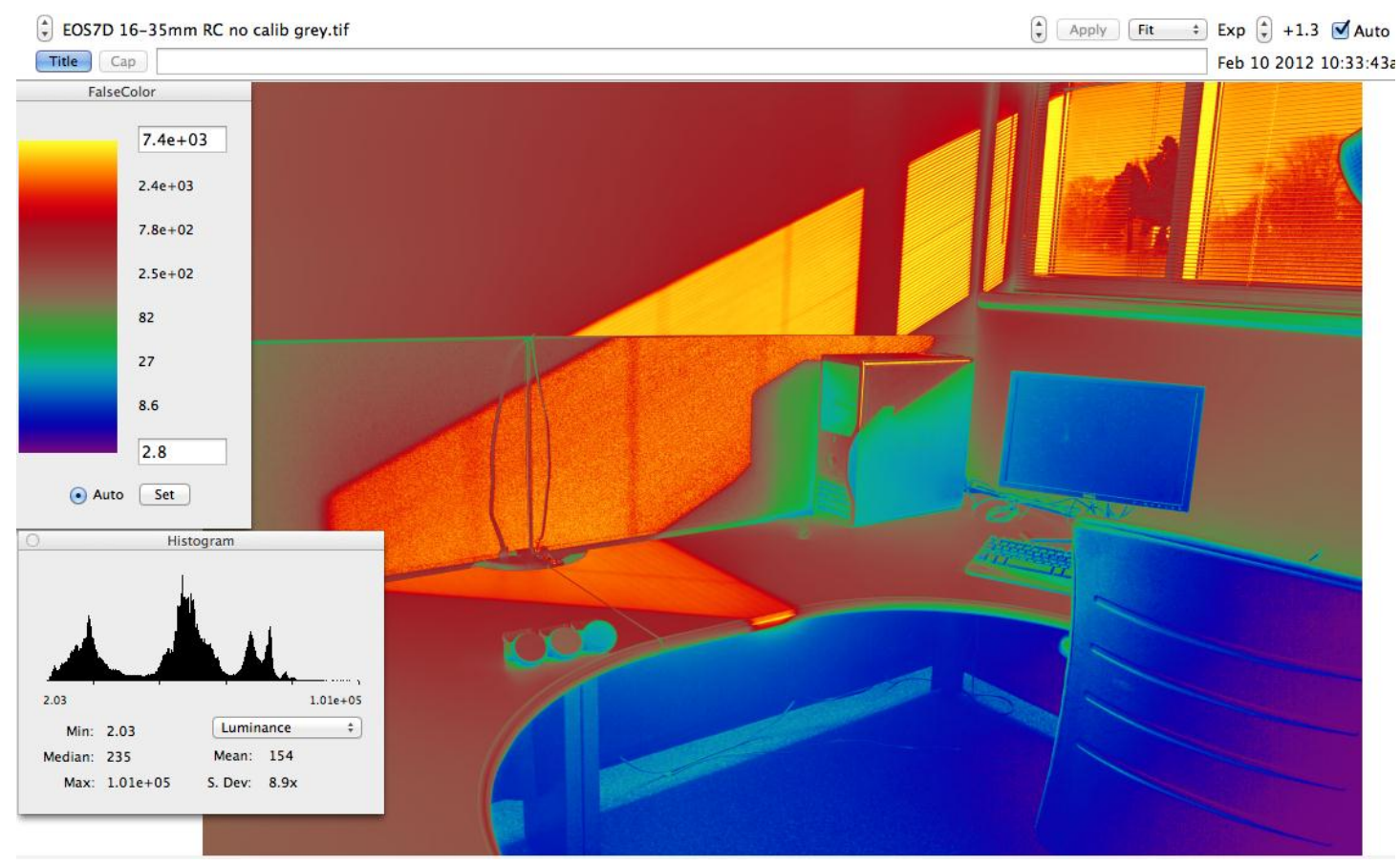

Figure 3-31. Pseudo colors HDRI of camera calibration scene for obtaining RC for EOS 7D camera fitted with CANON zoom lens 16-35 mm

LED luminance in the HDRI is underestimated (section 3.3.3.1). Such a big error (98\% compared to the calculated luminance in section 3.2.3) might occur because the scene didn't have very high luminance values for camera to sample.

In many scenes it is enough to give a wide range of luminances for camera to sample without providing extreme values. Extension to those extremes is not critical in most cases. Algorithm recovers the RC of the camera based on the given data. But 
it might be critical to give very high values for the camera to sample in case of capturing a bright light source.

Outcome: To try a calibration scene with a wider dynamic range for the purpose of obtaining the RC.

\subsubsection{Scene with a wider dynamic range}

Brighter scene will expand the luminance range for the camera to sample. The scene should include the sun, although, afternoon sun should not be used, because it will damage the sensor. In addition it is too bright. The chosen scene included the sun before the sunset. Measurements were taken on the 2/18/2012 at 4:21pm-4:25pm (figure 3-32).
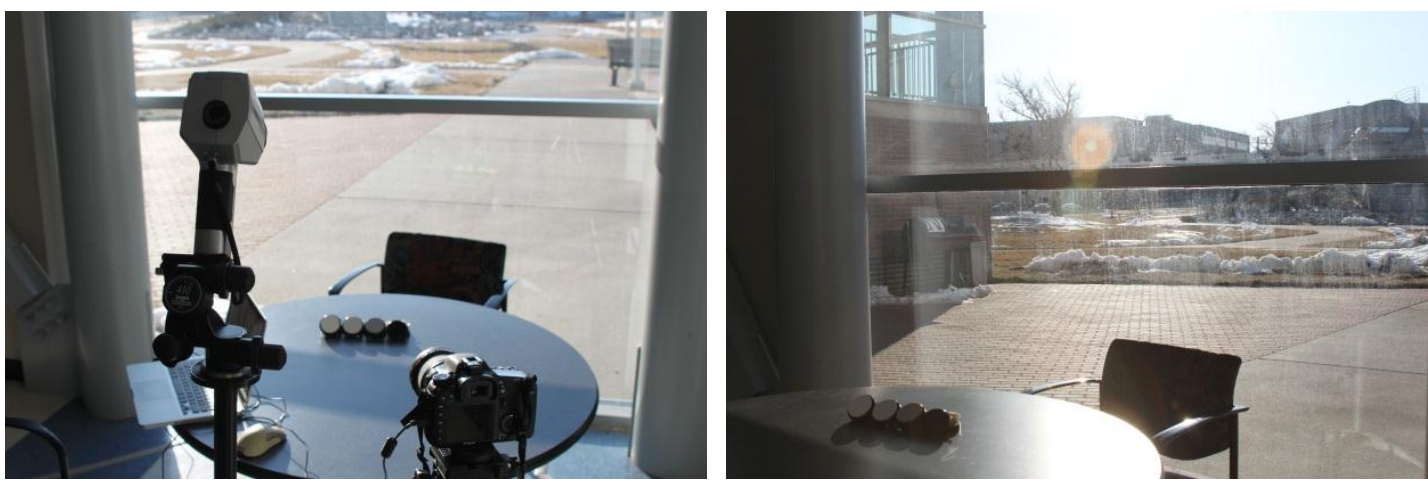

Figure 3-32. Calibration scene with a wider dynamic range for obtaining the RC of camera/lens combination

The results for EOS 7D digital camera at $16 \mathrm{~mm}$ focal length are shown in the tables 3-9 and 3-10. Table 3-9 shows measurements of reflectance standards with the LS110 luminance meter. Luminance values obtained from the HDRI without applying the CF in Photosphere are shown in table 3-10.

Table 3-9. Measurements with the LS110 luminance meter

\begin{tabular}{|c|c|c|c|c|}
\hline $\begin{array}{c}\text { Reflectance } \\
\text { standards/ } \\
\text { L, cd/m }\end{array}$ & White 99\% & White 80\% & Grey 40\% & Black 2\% \\
\hline $\begin{array}{c}\text { Before the } \\
\text { sequence of photos }\end{array}$ & 973.9 & 774.6 & 421.6 & 32.79 \\
\hline $\begin{array}{c}\text { After the sequence } \\
\text { of photos }\end{array}$ & 989.5 & 774.4 & 424.7 & 32.65 \\
\hline
\end{tabular}




\begin{tabular}{|c|c|c|c|c|}
\hline Average & 981.7 & 774.5 & 423.15 & 32.72 \\
\hline
\end{tabular}

Table 3-10. HDRI luminance values without applying the CF in Photosphere

\begin{tabular}{|c|c|c|c|c|c|c|}
\hline $\begin{array}{c}\text { Reflectance } \\
\text { standards/ } \\
\text { L, cd/m² }\end{array}$ & $\begin{array}{c}\text { White } \\
\mathbf{9 9 \%}\end{array}$ & $\begin{array}{c}\text { White } \\
\mathbf{8 0 \%}\end{array}$ & $\begin{array}{c}\text { Grey } \\
\mathbf{4 0 \%}\end{array}$ & $\begin{array}{c}\text { Black } \\
\mathbf{2 \%}\end{array}$ & $\begin{array}{c}\text { Maximum value } \\
\text { within captured } \\
\text { scene }\end{array}$ & $\begin{array}{c}\text { Minimum } \\
\text { value within } \\
\text { captured } \\
\text { scene }\end{array}$ \\
\hline $\begin{array}{c}\text { Luminance in } \\
\text { Photosphere } \\
\text { without } \\
\text { applying the } \\
\text { CF }\end{array}$ & 1260 & 1220 & 787 & 288 & 411000 & 0.342 \\
\hline
\end{tabular}

From the obtained results it is clearly seen that reflectance standards are overestimated, while the luminance for the Sun is underestimated. Calibration will not solve the problem since $\mathrm{CF}$ will scale all values by the same amount (appendix D).

Outcome: The scene with a wider dynamic range doesn't solve the problem. It is confirmed with the following experiment (not described in this work). The HDRI of LED fused with the RC obtained from this scene (with a wider dynamic range) is still low (the LED luminance value is $\mathbf{4 . 7 8} * \mathbf{1 0} \mathbf{5} \mathrm{cd} / \mathrm{m}^{2}$ ). Histograms of the shortest and longest exposures have to be checked.

\subsubsection{Exposures analysis with the histograms in Photoshop CS5}

Photoshop allows opening CR2 images (RAW image format for Canon digital cameras) without making adjustments to the color space. It allows viewing detailed histograms of the photo:

Image $>>$ Adjustments $>>$ Levels.

The shortest and the longest exposures were chosen from the sequence of the LED photographs used for fusing into the HDRI. They were checked in Photoshop CS5 (figures 3-33 to 3-36). 


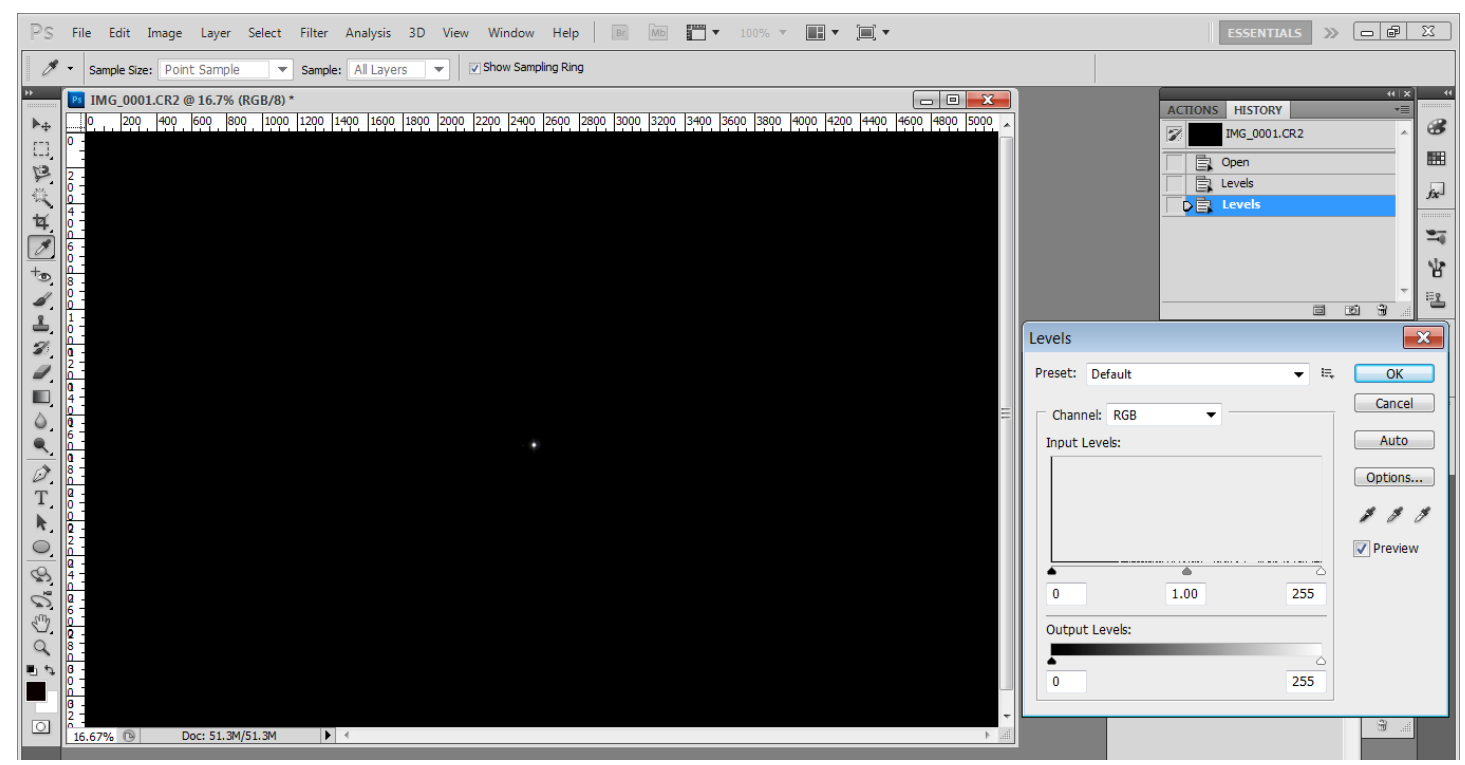

Figure 3-33. Histogram of the RGB values in the shortest exposure (1/8000'') of the LED sequence

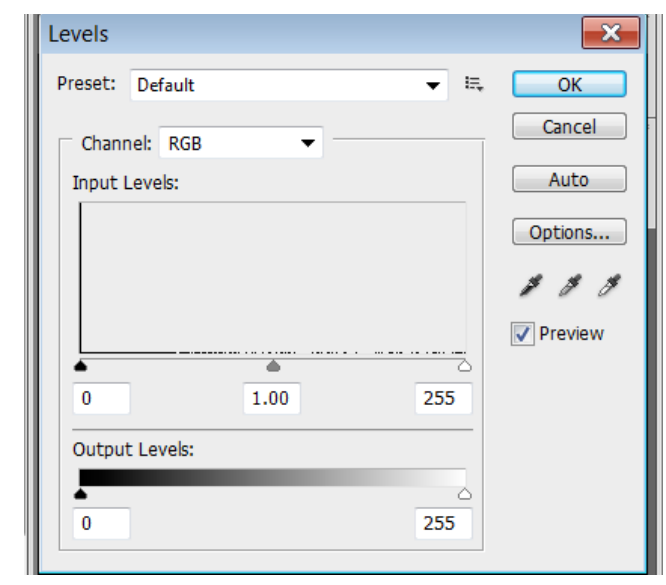

Figure 3-34. Enlarged histogram of the RGB values in the shortest exposure (1/8000', ) of the LED sequence 


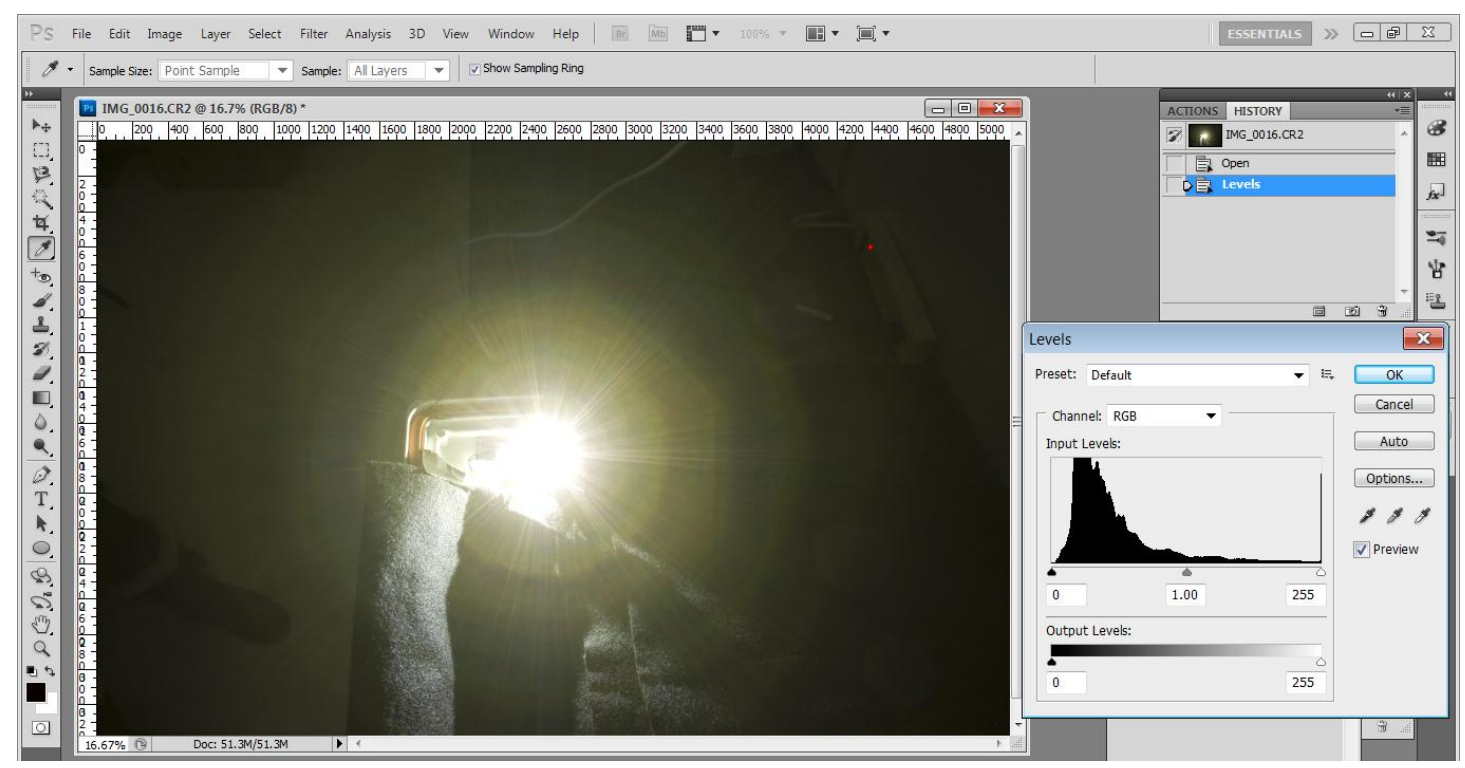

Figure 3-35. Histogram of the RGB values in the longest exposure (4,') of the LED sequence

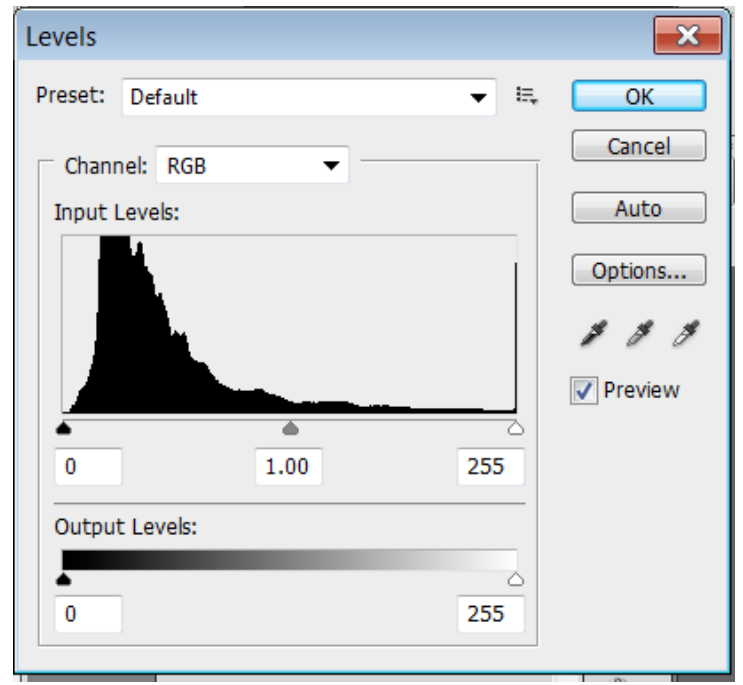

Figure 3-36. Enlarged histogram of the RGB values in the longest exposure (4',) of the LED sequence

According to section 2.2.1.2 Practical guidelines and appendix D, LED was not properly captured in this sequence.

Outcome: Technique to capture a single LED chip was determined. The LED should be properly captured with the neutral density (ND) filter and RAW images (see appendix D for more details). 
3.4 Measurements of a single LED chip in GE garage fixture with the HDRI technique

ND filter NDA2-703-002 with $\tau=0.0094$ that fits $28-105 \mathrm{~mm}$ lens was used to make an HDRI of the LED. CANON EOS7D camera was used out of two available cameras because it allows taking a photograph with the shortest exposure of 1/8000' compared to 1/4000', for EOS T1i Rebel CANON camera.

Response curve for this camera/lens combination had to be obtained and vignetting experiments had to be conducted.

\subsubsection{Response curve for Canon EOS 7D fitted with 28-105 mm CANON zoom lens}

Photographs for obtaining RC for Canon EOS 7D fitted with 28-105 mm CANON zoom lens were taken on the 3/5/2012 (table 3-11, figure 3-37). The procedure was similar to the one in section 3.3.1.

Table 3-11. Settings for the response curve experiments

\begin{tabular}{|c|c|}
\hline Camera & CANON EOS7D \\
\hline Lens & CANON zoom lens EF28-105mm 1:3.5-4.5 II USM \\
\hline $\begin{array}{c}\text { Luminance } \\
\text { meter }\end{array}$ & Minolta LS110 \\
\hline $\begin{array}{c}\text { Reflectance } \\
\text { standard }\end{array}$ & Labsphere (SRS-99-020, SRS-40-020,SRS-2-020), 99\%, 40\%, \\
\hline Scene & Interior daylighting, neutral colors, big smooth gradient, very \\
dark and very bright
\end{tabular}




\begin{tabular}{l|l} 
Mode & Manual
\end{tabular}

$\mathrm{RC}$ coefficients:

$\operatorname{red}(\mathrm{r})=5.344807 \mathrm{e}-03+-6.434375 \mathrm{e}-02 * \mathrm{r}+2.280451 \mathrm{e}+00 * \mathrm{r}^{\wedge} 2+-$

$5.606413 \mathrm{e}+00 * \mathrm{r}^{\wedge} 3+4.404040 \mathrm{e}+00 * \mathrm{r}^{\wedge} 4$

$\operatorname{green}(\mathrm{g})=4.634515 \mathrm{e}-03+-2.500448 \mathrm{e}-02 * \mathrm{~g}+1.946005 \mathrm{e}+00^{*} \mathrm{~g} \wedge 2+$

$-4.784106 e+00 * g^{\wedge} 3+3.877550 e+00 * g^{\wedge} 4$

blue $(b)=3.387779 e-03+-2.147793 e-02 * b+1.796577 e+00 * b^{\wedge} 2+-$

$4.638576 e+00 * b^{\wedge} 3+3.879167 e+00 * b^{\wedge} 4$.

Then hand-held calibration was made with the LS110 luminance meter at the grey standard $\left(353.8 \mathrm{~cd} / \mathrm{m}^{2}\right)$. CF is 1.019 .

Table 3-12 Measurements of reflectance standards with LS110 luminance meter

\begin{tabular}{|c|c|c|c|}
\hline $\begin{array}{c}\text { Reflectance } \\
\text { standard } \\
\text { measurements with } \\
\text { the luminance } \\
\text { meter, cd/m }\end{array}$ & White 99\% & Grey 40\% & Black 2\% \\
\hline $\begin{array}{c}\text { Before the sequence } \\
\text { of photos }\end{array}$ & 672.6 & 351.1 & 23.75 \\
\hline $\begin{array}{c}\text { After the sequence } \\
\text { of photos }\end{array}$ & 681.6 & 356.5 & 24.55 \\
\hline Average & 677.1 & 353.8 & 24.15 \\
\hline
\end{tabular}

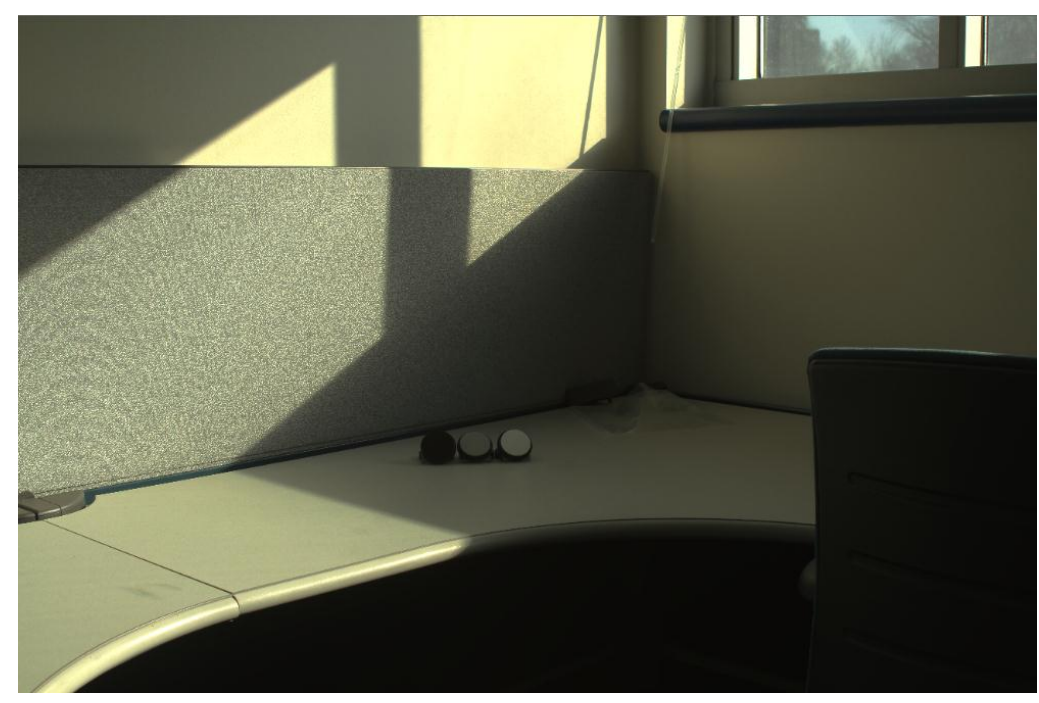

Figure 3-37. Experimental set-up for deriving the response curve for EOS 7D and 28-105 CANON zoom lens combination 
Outcome: After obtaining response curve and making hand-held calibration with the luminance meter, camera can be used as a luminance mapping device.

\subsubsection{Using raw2hdr Perl script for fusing a sequence of images}

The outcome of determining experimental conditions to capture the LED is to use RAW images and neutral density filter. A new approach uses raw2hdr in command-line tool on Macintosh computer (the script is written by G. Ward). The Perl script uses dcraw with the command-line HDR image builder hdrgen, and also requires the use of exiftool. It allows fusing RAW images into an HDR image (Photosphere doesn't accept RAW format). Then obtained HDRI was analyzed in Photosphere, where it can be calibrated (refer to appendix D - HDRI mailing lists, appendix E for Perl script settings and anyhere software website).

In order to fuse a sequence of images the following line has to be typed in the command-line of the Mac Terminal (see appendix E for settings):

raw2hdr $-\mathrm{f}-\mathrm{g}-\mathrm{o}$ image.hdr IMG_0???.CR2

Digital images undergo many transformations during their lifetime (Reinhard et al. 2010). RAW format can be compared to film photography as being photography's digital equivalent of a negative. This format contains all "raw" pixel information from the digital camera's sensor. Sequence of adjustments has to be made for RAW format to be "developed" to JPEG or TIFF. Those adjustments include: demosaicing, white balance, tone curves, contrast, color saturation, sharpening, conversion to 8-bit JPEG compression (lossy compression - image sizes are small, some image data is discarded), TIFF compression (lossless compression - info is preserved, it can be 16 bits per channel) (according to Cambridge in Color Website as of September 2011). JPEG processing has inherent color issues. RAW files bypass the problems of correction manipulations entirely because the data is linear (appendix E). 
Working with RAW images is a reasonable way to get around most of the in-camera processing that undermines absolute CIE color accuracy (HDRI mailing lists as of September 2006).

\subsubsection{The HDRI capture of an incandescent light source}

The accuracy of measurements of a light source in the scene that can be obtained with raw2hdr was tested by capturing an incandescent light source (see appendix F). The results give confidence in using this approach.

\subsubsection{Optical vignetting effect}

The results of optical vignetting experiments were obtained for two software (Photosphere with JPEG images and raw2hdr Perl script with RAW). Then vignetting effect was analyzed for the area of the image where LED and reflectance standard were located. Experiments were conducted with the ULS same as in section 3.3.2.

\subsubsection{Comparing optical vignetting effect experiment results for two software}

Goal: To compare luminance measurement error due to optical vignetting effect for RAW images (raw2hdr Perl script) and JPEG images (Photosphere).

Four HDR images were obtained for the purpose of this experiment. Camera EOS 7D with CANON zoom lens 28-105 mm was used. Photographs were made with two aperture sizes (f/4.5 and f/16) at $105 \mathrm{~mm}$ zoom. JPEG Images were fused in Photosphere with the known RC for the camera/lens combination and RAW images were merged with raw2hdr Perl script. HDRIs were calibrated in Photosphere.

Then nine areas (300x280 pixels) of each HDRI were analyzed (figures 3-38 and 3-39).

Mean values of nine areas of each HDRI were obtained from Photosphere and put onto one graph (table 3-13, figure 3-40). 
$8.47 \mathrm{e}+03 \mathrm{~cd} / \mathrm{m} 2(2369,1732) /(2674,1967)$

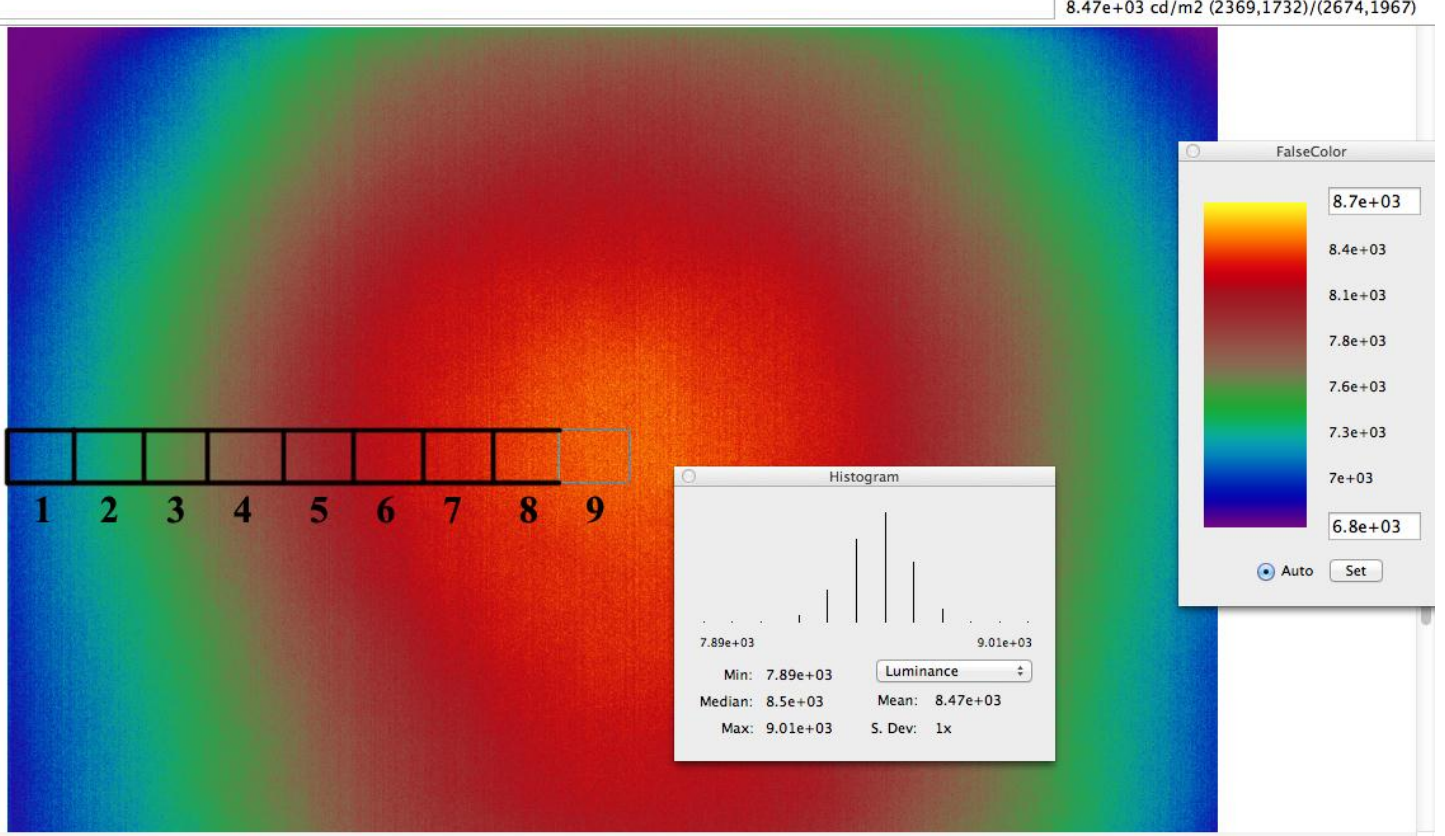

Figure 3-38. Nine areas $(300 \times 280$ pixels $)$ used for the vignetting analysis

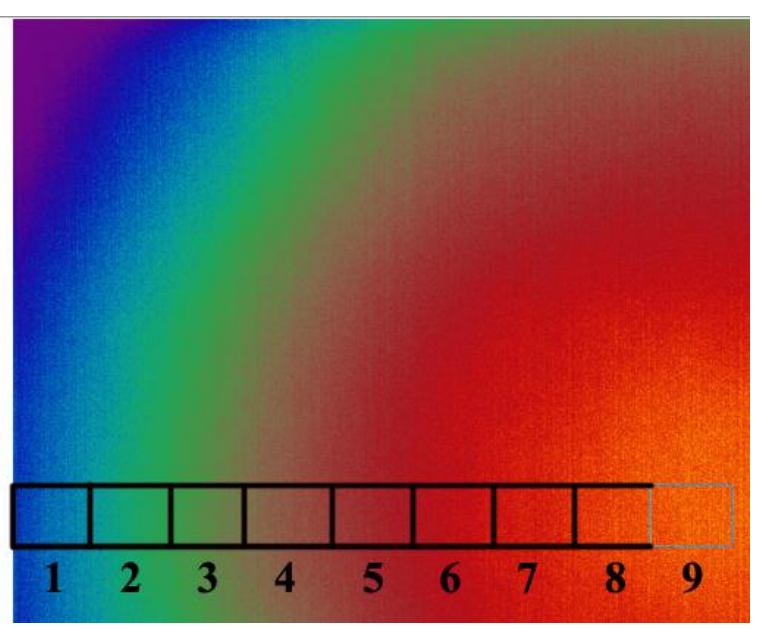

Figure 3-39. $1 / 4^{\text {th }}$ of an image for the vignetting analysis 
Table 3-13. Mean, minimum, maximum values and average standard deviation of HDRIs fused with RAW and JPEG images

\begin{tabular}{|c|c|c|c|c|c|c|c|c|c|c|c|c|}
\hline \multirow[t]{2}{*}{$\begin{array}{c}\text { Position } \\
\text { on the } \\
\text { image }\end{array}$} & \multicolumn{3}{|c|}{$\begin{array}{c}\text { Fused with Hdrgen } \\
\text { @ } 105 \mathrm{~mm} \\
\text { aperture } 4.5\end{array}$} & \multicolumn{3}{|c|}{$\begin{array}{c}\text { Fused with Hdrgen } \\
\text { @ } 105 \text { mm } \\
\text { aperture } 16\end{array}$} & \multicolumn{3}{|c|}{$\begin{array}{c}\text { Fused with } \\
\text { Photosphere @ } 105 \\
\text { mm aperture } 4.5\end{array}$} & \multicolumn{3}{|c|}{$\begin{array}{c}\text { Fused with } \\
\text { Photosphere @ } 105 \\
\text { mm aperture } 16\end{array}$} \\
\hline & Mean & Min & Max & Mean & Min & Max & Mean & Min & Max & Mean & Min & Max \\
\hline 1 & 7170 & 6690 & 7590 & 7850 & 7240 & 8300 & 6770 & 6210 & 7640 & 7420 & 6690 & 8830 \\
\hline 2 & 7430 & 6960 & 7890 & 7980 & 7380 & 8360 & 7060 & 6260 & 7760 & 7660 & 6770 & 8970 \\
\hline 3 & 7630 & 7070 & 8080 & 8080 & 7380 & 8590 & 7340 & 6610 & 8290 & 7830 & 6900 & 9180 \\
\hline 4 & 7850 & 7270 & 8300 & 8200 & 7580 & 8660 & 7630 & 6850 & 8790 & 8000 & 7120 & 9070 \\
\hline 5 & 8040 & 7470 & 8500 & 8290 & 7580 & 8730 & 7910 & 7150 & 9030 & 8140 & 7070 & 9620 \\
\hline 6 & 8200 & 7680 & 8600 & 8360 & 7790 & 8730 & 8110 & 7400 & 9030 & 8260 & 7260 & 9550 \\
\hline 7 & 8310 & 7770 & 8800 & 8390 & 7790 & 8870 & 8260 & 7290 & 9180 & 8300 & 7380 & 9620 \\
\hline 8 & 8420 & 7890 & 9010 & 8440 & 7880 & 9040 & 8380 & 7640 & 9180 & 8360 & 7380 & 9260 \\
\hline 9 & 8470 & 7890 & 9010 & 8480 & 7880 & 9180 & 8460 & 7640 & 9510 & 8480 & 7430 & 9400 \\
\hline $\begin{array}{l}\text { Average } \\
\text { SD for } 9 \\
\text { positions }\end{array}$ & \multicolumn{3}{|c|}{109} & \multicolumn{3}{|c|}{113} & \multicolumn{3}{|c|}{101} & \multicolumn{3}{|c|}{115} \\
\hline
\end{tabular}




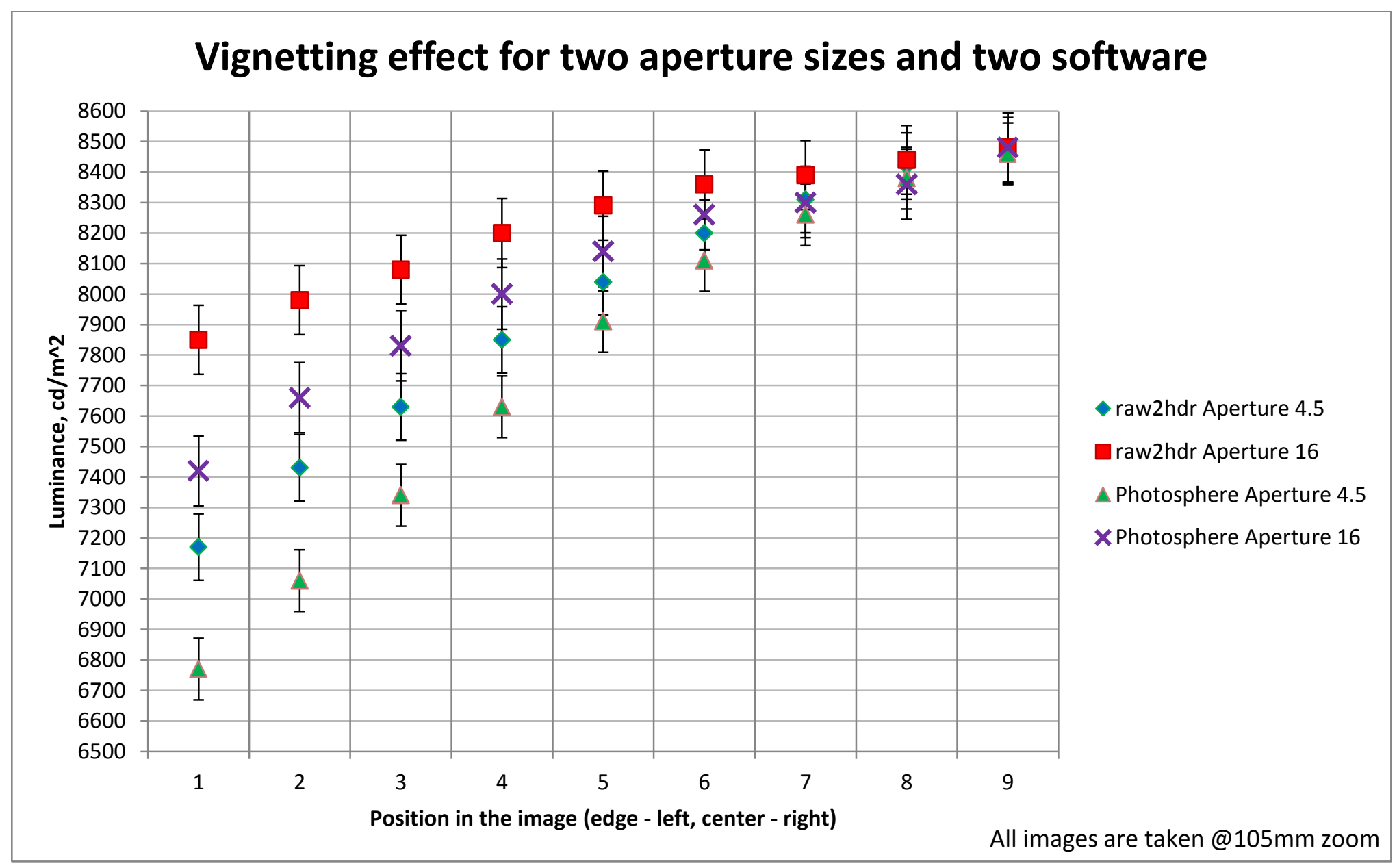

Figure 3-40. Mean luminance values and standard deviations for 9 zones of four HDRIs (two are fused in Photosphere and - two in raw2hdr) 
Outcome: For the same aperture size optical vignetting effect is smaller for the HDRI created with RAW images. More detailed research on vignetting effect for different software, aperture sizes and other variables can be performed in a future work.

\subsubsection{Analysis of the critical area of the HDRI where LED and reflectance standard's calibration zone are located}

Vignetting effect was analyzed for the area of the image where the LED and reflectance standard's calibration zone are located (figures 3-41 and 3-42). Experiments were conducted with ULS as in section 3.3.2. The size of the selected area can be observed in the upper right corner in Photosphere (pixels). RAW images of the ULS were taken with two different aperture sizes (f/4.5 and f/16) at $105 \mathrm{~mm}$ focal length and fused with raw2hdr.

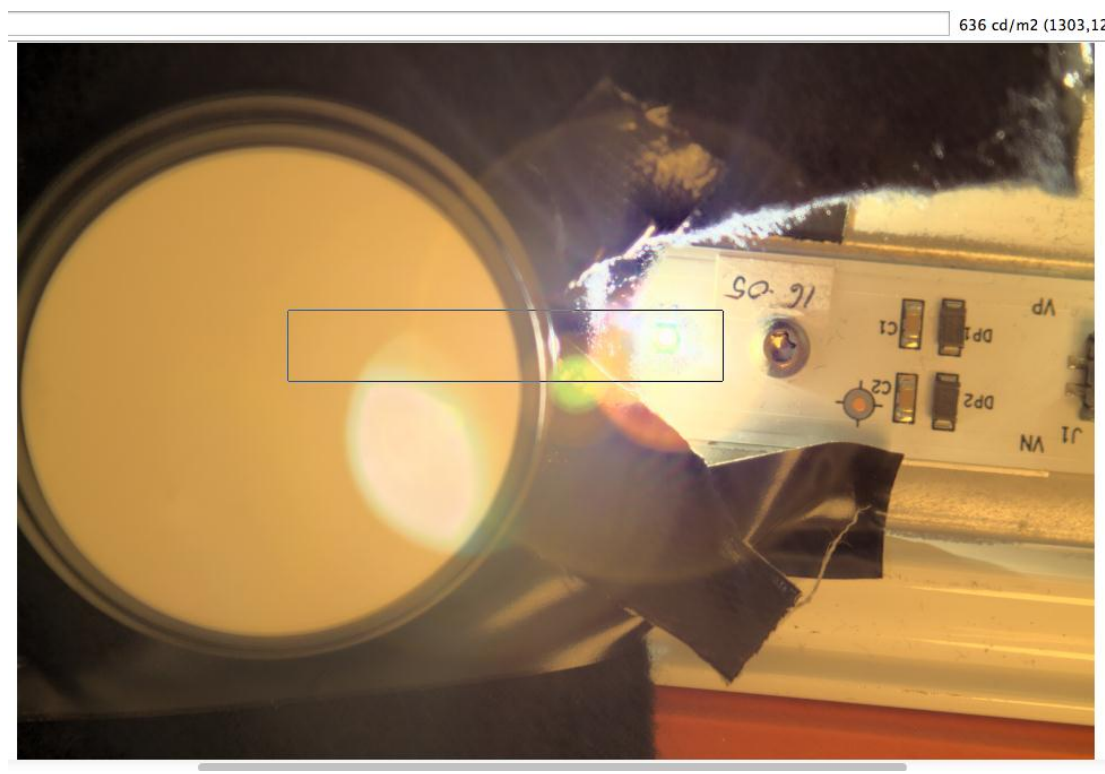

Figure 3-41. Part of the image with locations of the LED and reflectance standard's calibration zone 


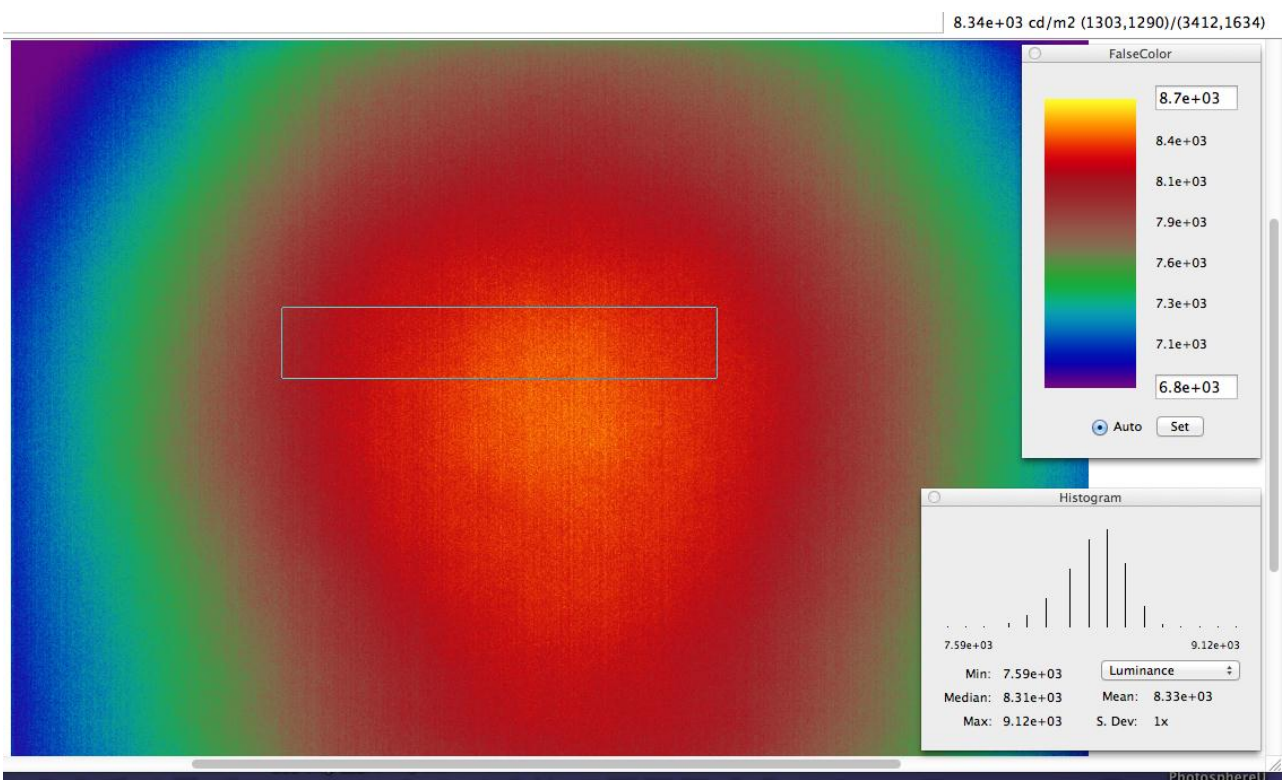

Figure 3-42. HDRI of ULS with the area of the LED and reflectance standard's calibration zone's locations (in pseudo colors)

The position of the LED and reflectance standard's calibration zone were

estimated based on pixel locations. LED is located in the area 7 on the other half of the image; while reflectance standard's calibration zone is located in area 5 (figure 343).

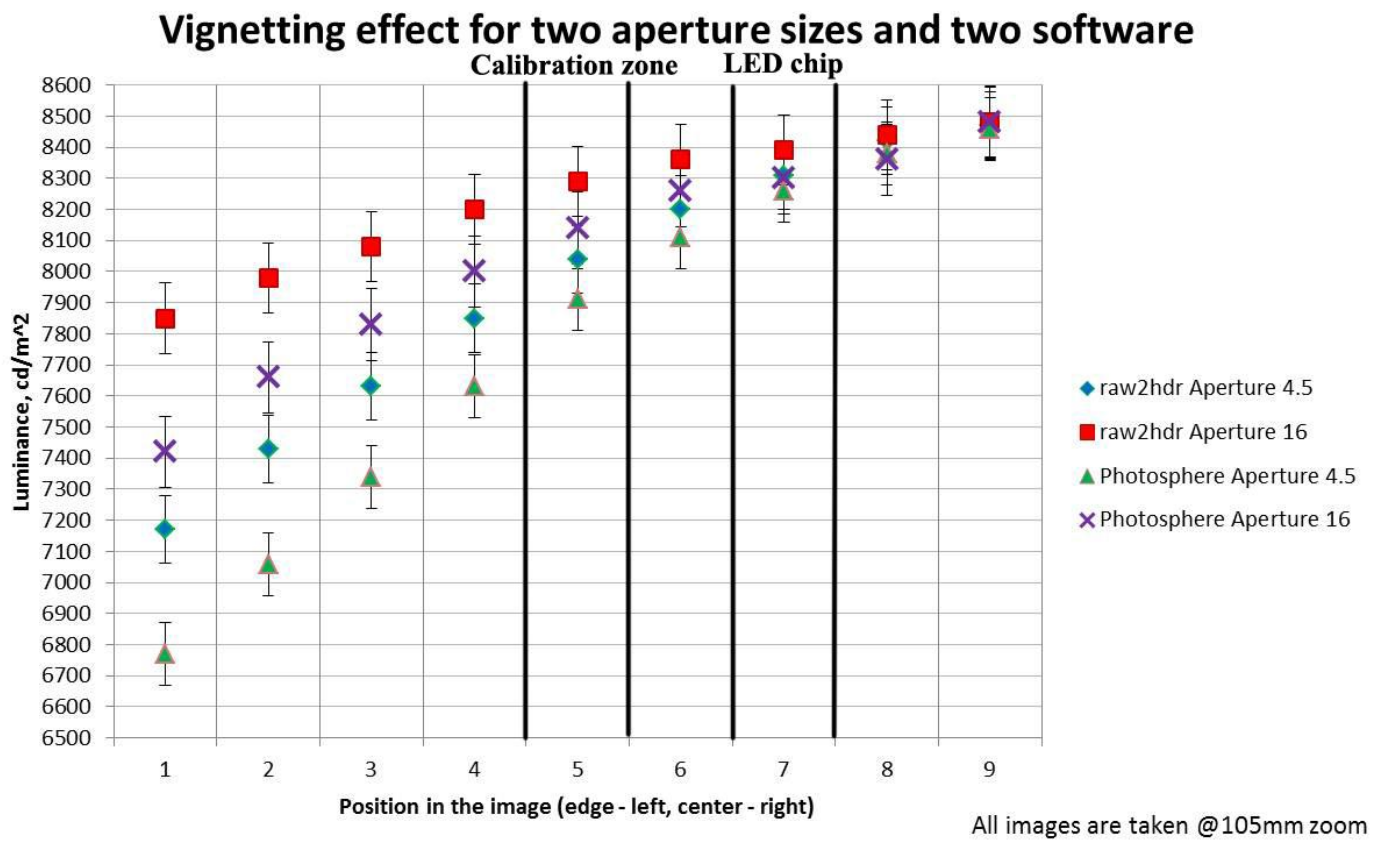

Figure 3-43. Mean luminance values and standard deviations for 9 zones of four HDRIs of the LED and reflectance standard calibration zone's location 
Figures 3-44 - 3-47 show HDRIs of ULS with locations of LED and reflectance standard's calibration zone.

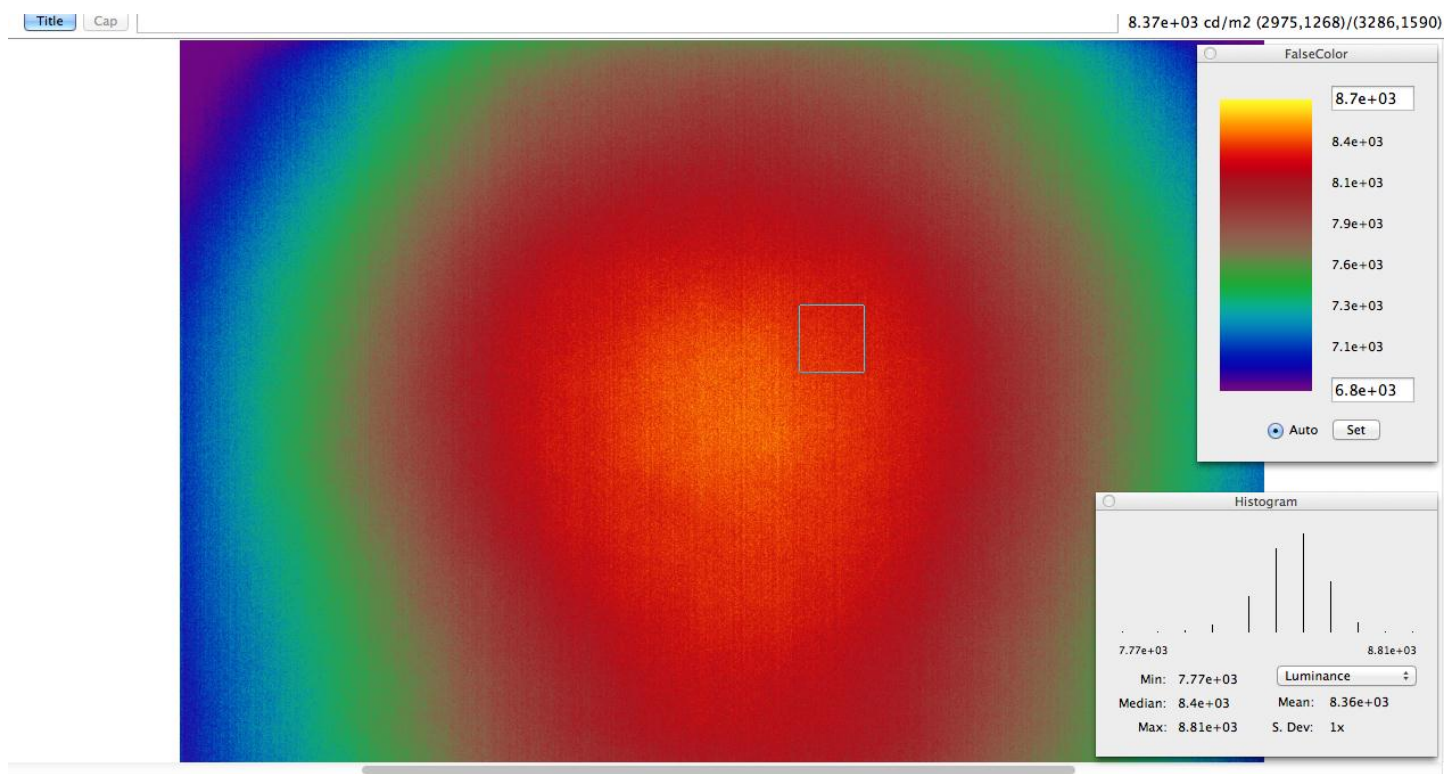

Figure 3-44. The LED location on the image testing optical vignetting effect (aperture 4.5, fused in raw $2 \mathrm{hdr} @ 105 \mathrm{~mm}$ )

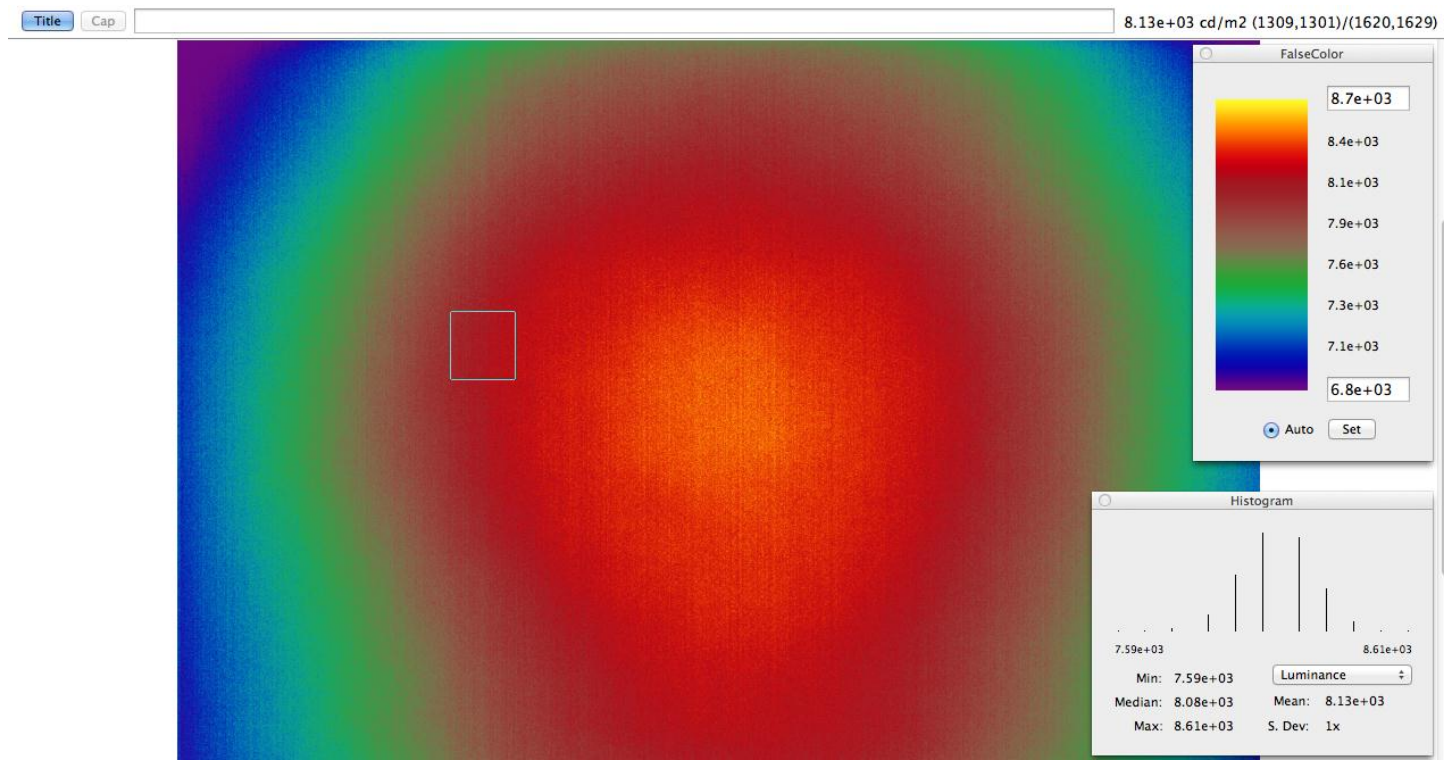

Figure 3-45. Reflectance standard's calibration zone location on the image testing optical vignetting effect (aperture 4.5, fused in raw2hdr @105mm) 


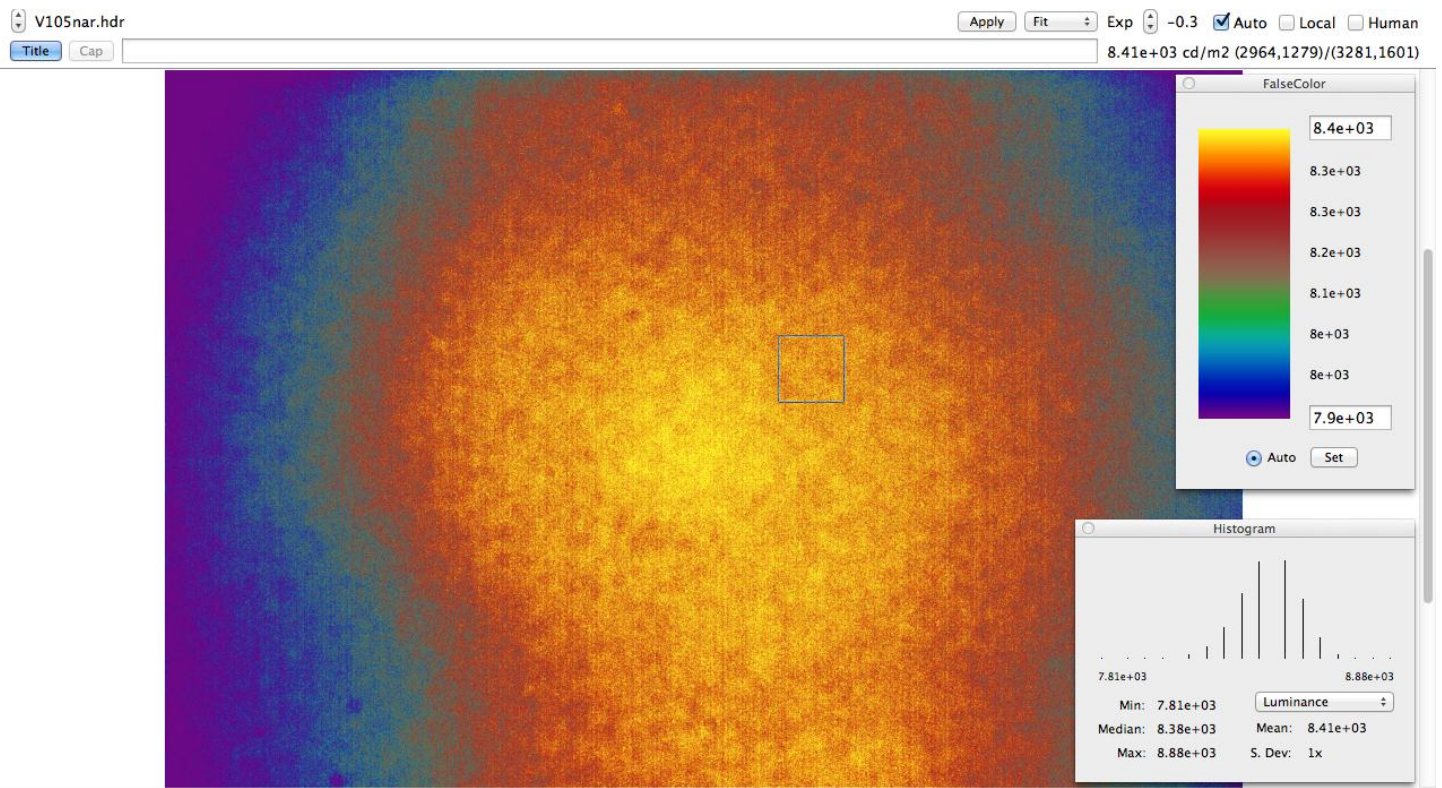

Figure 3-46. The LED location on the image testing optical vignetting effect (aperture 16, fused in raw $2 \mathrm{hdr} @ 105 \mathrm{~mm}$ )

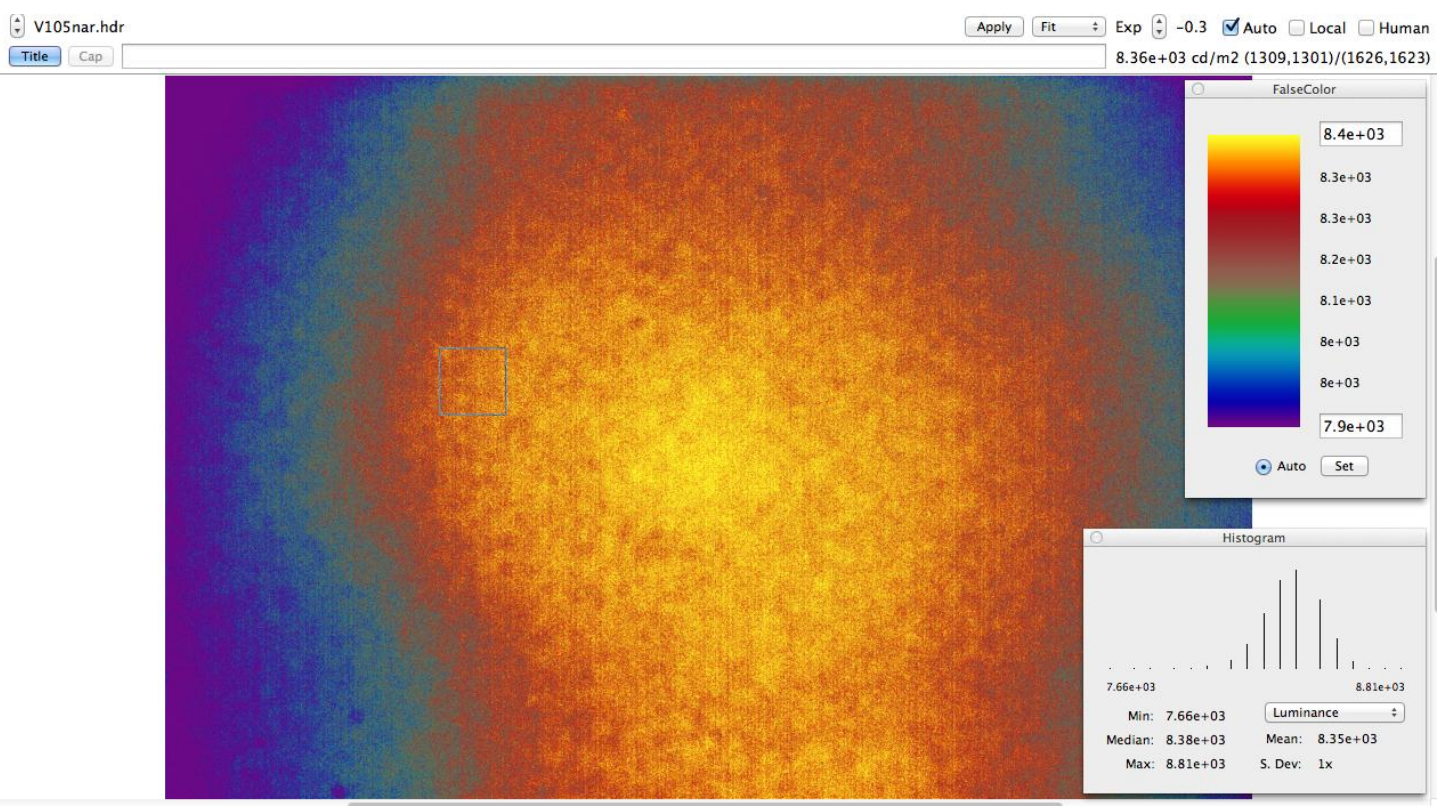

Figure 3-47. Reflectance standard's calibration zone location on the image testing optical vignetting effect (aperture 16, fused in raw $2 \mathrm{hdr}$ @105mm)

To calculate the error between luminance values due to the optical vignetting effect (HDRI of ULS), mean values of areas with locations of the LED and reflectance standard's calibration zone were used (table 3-14).

$$
\text { Error }=\frac{\mid \text { Zone } 7-\text { Zone } 5 \mid}{\text { Zone } 7} \times 100 \%
$$


Table 3-14. Mean values used for calculating the luminance error due to the optical vignetting effect (in red)

\begin{tabular}{|c|c|c|c|c|c|c|}
\hline $\begin{array}{c}\text { Position } \\
\text { on the } \\
\text { image }\end{array}$ & \multicolumn{3}{|c|}{$\begin{array}{c}\text { Fused with Hdrgen } \\
\text { @ 105mm aperture }\end{array}$} & \multicolumn{3}{c|}{$\begin{array}{c}\text { Fused with Hdrgen } \\
\text { 4.5 105mm aperture } \\
\text { 16 }\end{array}$} \\
\hline & Mean & Min & Max & Mean & Min & Max \\
\hline $\mathbf{1}$ & 7170 & 6690 & 7590 & 7850 & 7240 & 8300 \\
\hline $\mathbf{2}$ & 7430 & 6960 & 7890 & 7980 & 7380 & 8360 \\
\hline $\mathbf{3}$ & 7630 & 7070 & 8080 & 8080 & 7380 & 8590 \\
\hline $\mathbf{4}$ & 7850 & 7270 & 8300 & 8200 & 7580 & 8660 \\
\hline $\mathbf{5}$ & $\mathbf{8 0 4 0}$ & 7470 & 8500 & $\mathbf{8 2 9 0}$ & 7580 & 8730 \\
\hline $\mathbf{6}$ & 8200 & 7680 & 8600 & 8360 & 7790 & 8730 \\
\hline $\mathbf{7}$ & $\mathbf{8 3 1 0}$ & 7770 & 8800 & $\mathbf{8 3 9 0}$ & 7790 & 8870 \\
\hline $\mathbf{8}$ & 8420 & 7890 & 9010 & 8440 & 7880 & 9040 \\
\hline $\mathbf{9}$ & 8470 & 7890 & 9010 & 8480 & 7880 & 9180 \\
\hline
\end{tabular}

For the aperture $\mathrm{f} / 4.5$ the error equals to $3.2 \%$, for aperture $\mathrm{f} / 16$ - error $1.2 \%$

Outcome: For the purpose of this research vignetting effect is not taking into consideration since all the HDRI measurements are taken in the parts of the image, where luminance error due to vignetting effect is less than $5 \%$.

\subsubsection{Capturing a single LED chip with the ND filter in RAW format and fusing} images with raw2hdr Perl script

\subsubsection{Experimental setting}

The LED was captured with CANON EOS 7D fitted with 28-105 mm at 105 $\mathrm{mm}$ focal length. If to use only one aperture size (for example f/16) while capturing the LED chip on the long exposure end (so that there are no RGB values below 28), exposure time has to be too long (more than 5 minutes for one photograph). This is unacceptable for making images of fast-changing lighting environments (for e.g. the sun and sky). In this experiment two aperture sizes were used (f/4.5 and f/16). Similar approach is used in literature (Stumphel et al. 2004, Inanici 2010). Shutter speeds used were $1 / 8000-1 / 15^{\prime \prime}$ at the aperture size $\mathrm{f} / 16$, and 1/30-30', at the aperture size f/4.5. Neutral density filter NDA2-703-002 with $\tau=0.0094$ was used. Camera was normal to the LED (figure 3-48). Image sequence was fused in raw2hdr (appendix E) 
and calibrated in Photosphere at white reflectance standard $(\rho=99 \%)=155.3 \mathrm{~cd} / \mathrm{m}^{2}$

(figure 3-49).
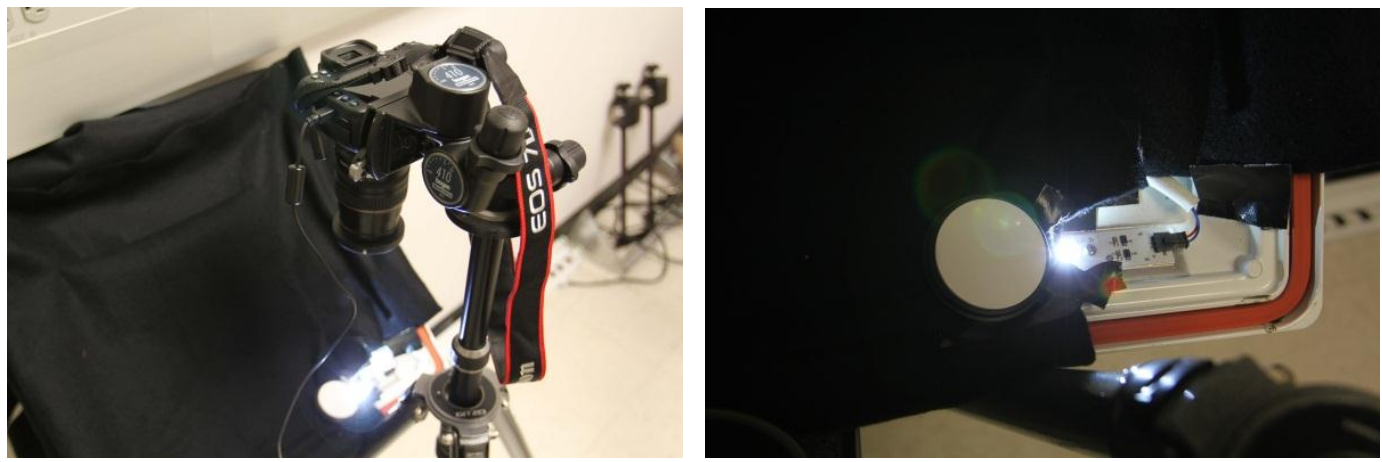

Figure 3-48. Experimental setting for making an HDRI of a single LED chip

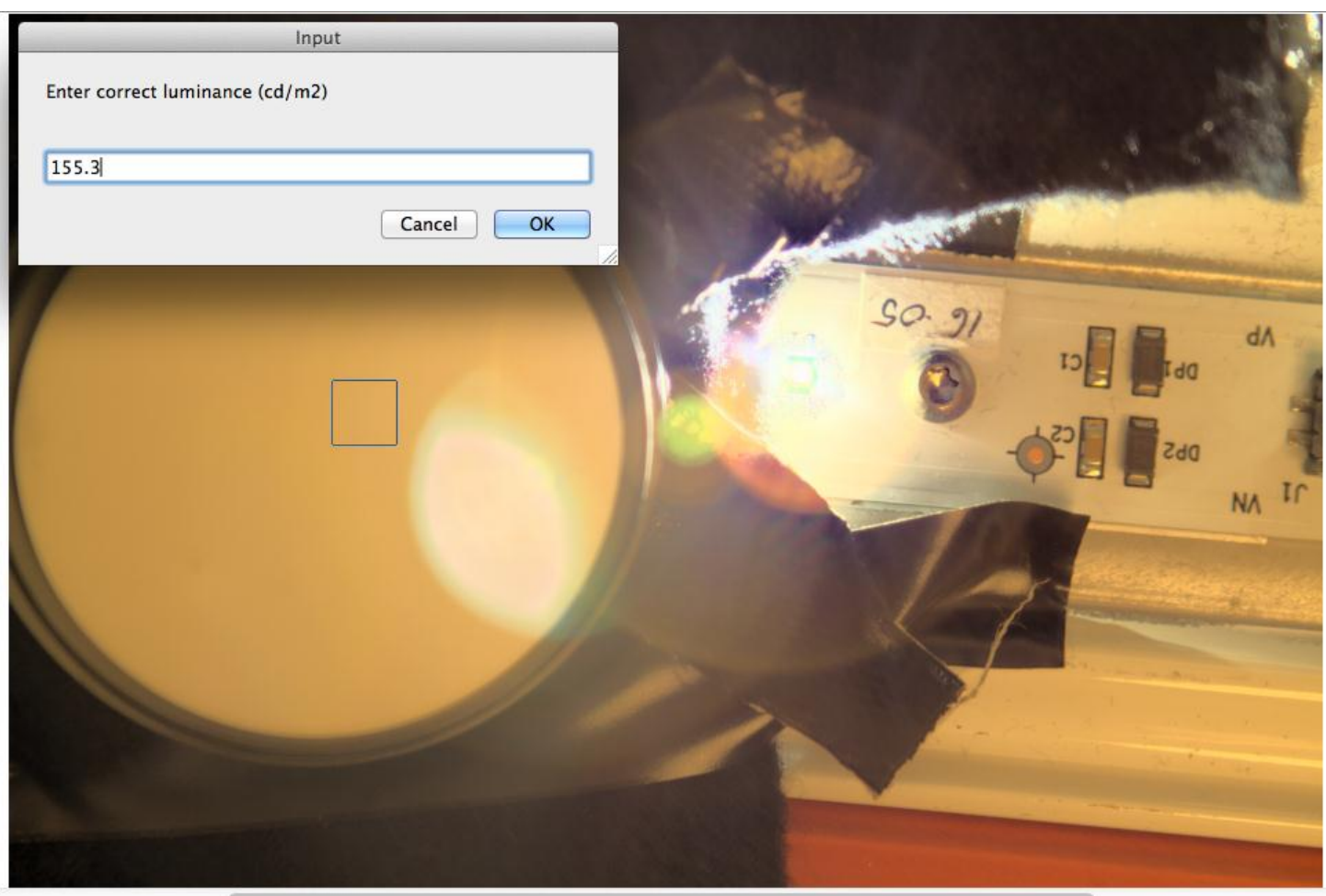

Figure 3-49. HDR image of a single LED chip and reflectance standard $(\rho=99 \%)$. Calibration at white reflectance standard 


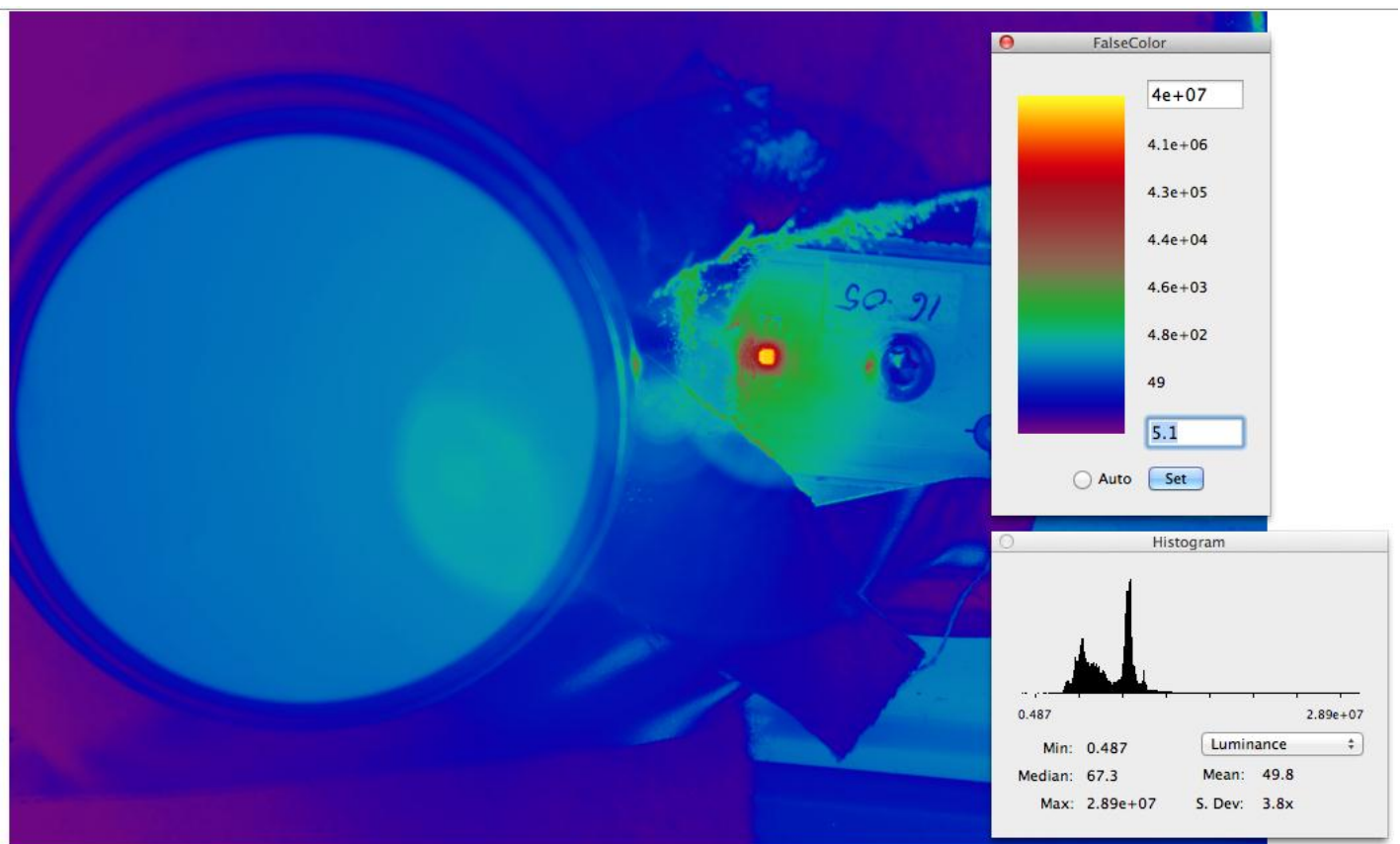

Figure 3-50. HDR image of a single LED chip and reflectance standard $(\rho=99 \%)$ in pseudo colors

To make sure that there are no RGB values lower than 28 for the longest exposure (RAW format) and no values higher than 227 for the shortest, histograms were checked in Photoshop CS5 (figures 3-51 - 3-54).

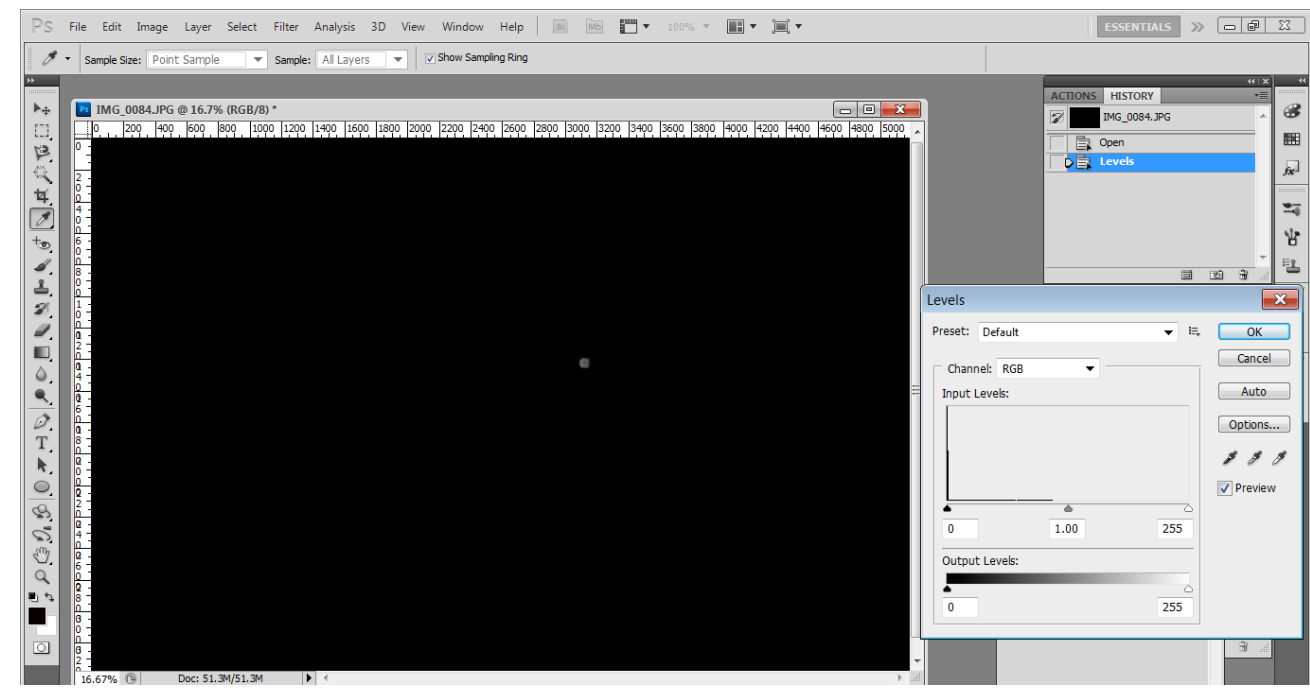

Figure 3-51. Histogram of the RGB values of the shortest exposure (1/8000', f/16, ND NDA2-703-002) in a single LED sequence 


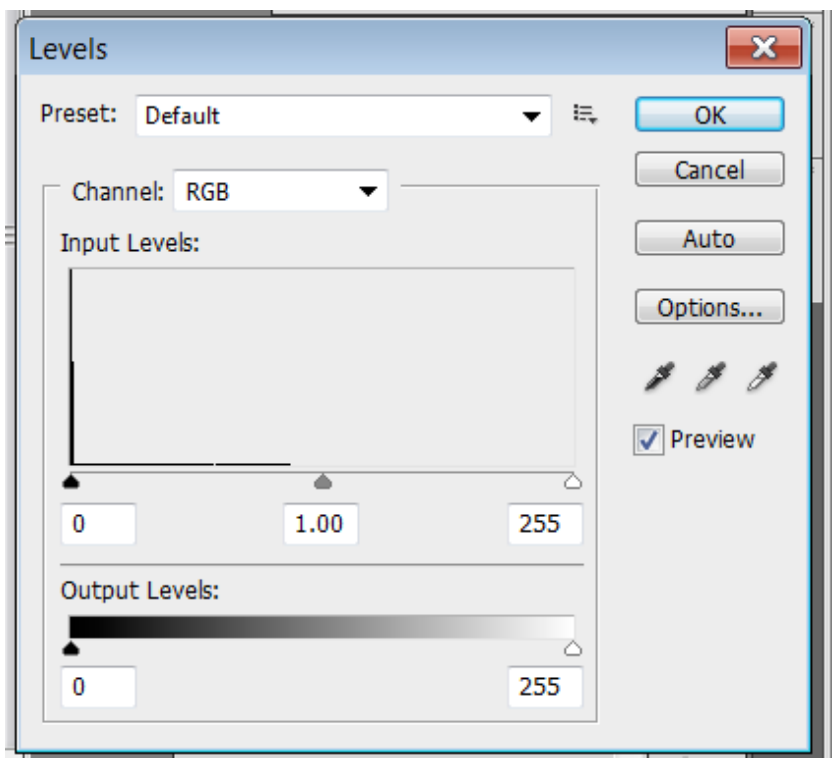

Figure 3-52. Enlarged histogram of the RGB values in the shortest exposure (1/8000', f/16, ND NDA2-703-002) in a single LED sequence

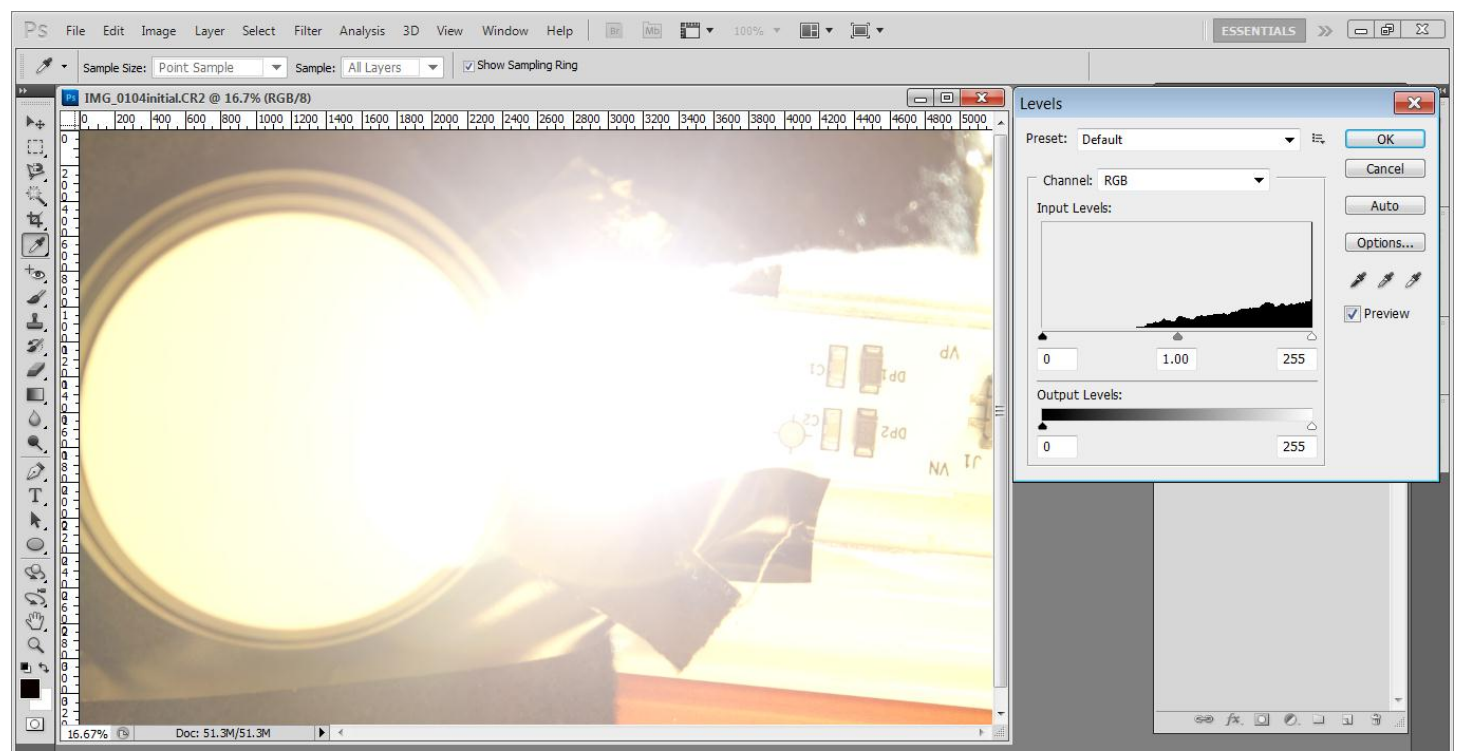

Figure 3-53. Histogram of the RGB values in the longest exposure (30', f/4.5, ND NDA2-703-002) in a single LED sequence 


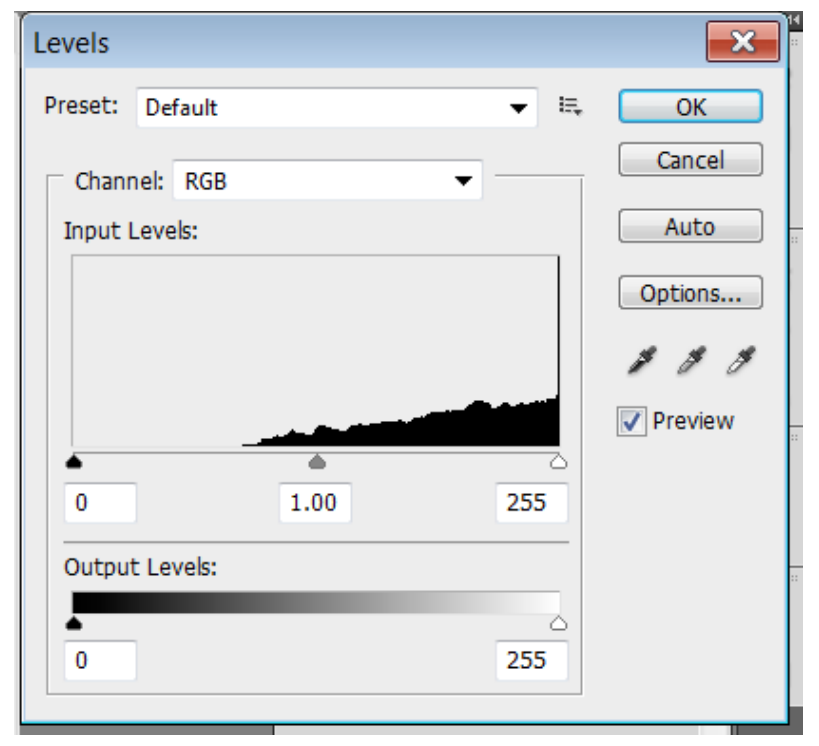

Figure 3-54. Enlarged histogram of the RGB values in the longest exposure (30', f/4.5, ND NDA2-703-002) in a single LED sequence

From figures 3-52 and 3-54, for the shortest exposure all RGB values are below 127.5, and the longest exposure histogram doesn't have values below 70 .

Outcome: Histograms show that sequence used for the HDRI acquisition was captured properly, now it can be fused for further analysis.

\subsubsection{Analysis of average luminance measurements with the HDRI}

Image sequence was fused with raw2hdr and calibrated in Photosphere at white reflectance standard $(\rho=99 \%)=155.3 \mathrm{~cd} / \mathrm{m}^{2}$ (figure 3-49). Then LED chip was enlarged, and HDRI was viewed in pseudo colors (figure 3-55).

Depending on the area within which the measurement is averaged in Photosphere, the readings change dramatically.

Mean value obtained for the selected area smaller than the LED chip is $2.04 * 10^{7} \mathrm{~cd} / \mathrm{m}^{2}$ (figure $3-55$ ).

The size of the LED chip in the image is 48 pixels $\mathrm{x} 48$ pixels (Photosphere). The area that LS110 luminance meter measures is $23 \%$ more than the size of LED chip (section 3.2.2). It corresponds to a size of about 53 pixels $x 53$ pixels in the image. 
Figures 3-56(A-E) show the mean luminance values when this area is moved around the LED chip. It can be compared with the value measured with LS110 and the close-up lens.

Aiming at the LED with the luminance meter is very difficult (section 3.2.2). This unreliable result is confirmed by luminance values obtained from the HDRI when selected area is moved around the LED chip (it measures LED with the borders outside of the chip). The range of mean values is $(7.46-9.85) * 10^{6} \mathrm{~cd} / \mathrm{m}^{2}$.

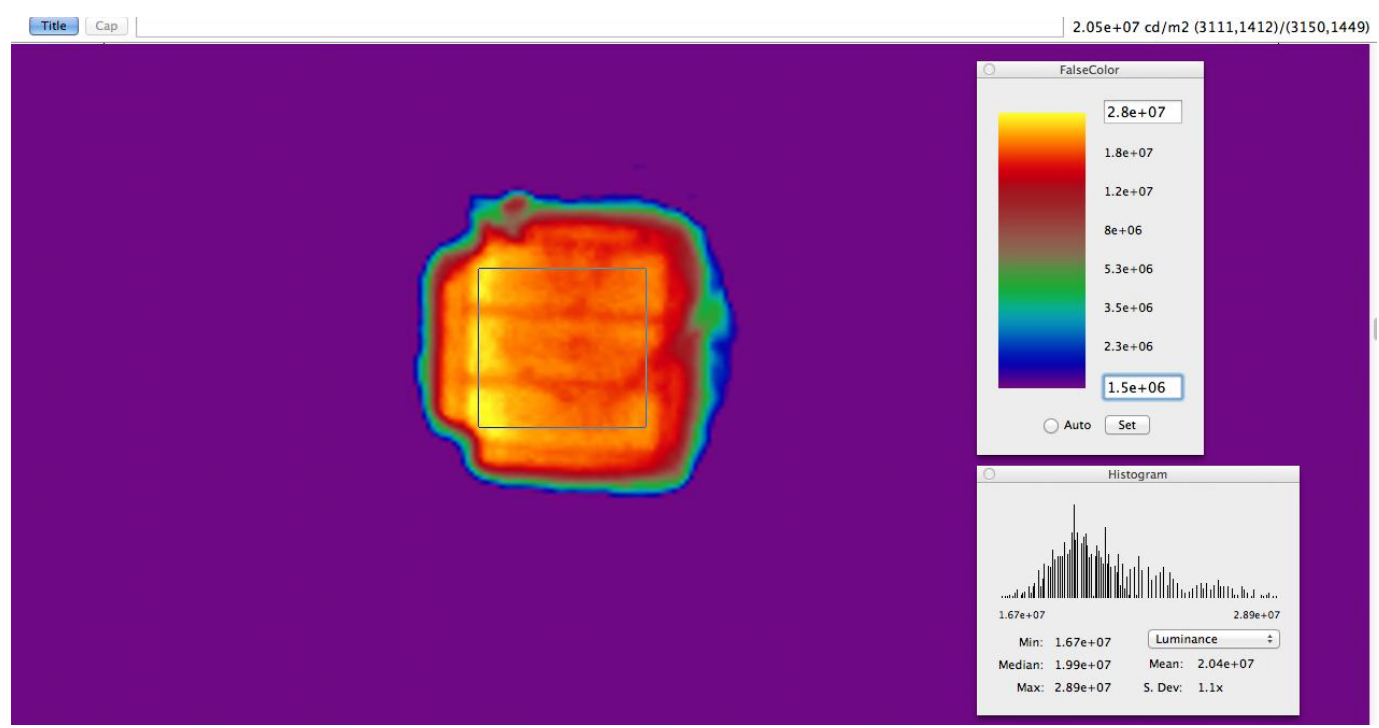

Figure 3-55. Selected area $(39 \times 37$ pixels) is smaller than the LED chip $(1.4 \mathrm{~mm} \times 1.4 \mathrm{~mm}, 48$ pixels x 48 pixels $)$. Mean value $2.04 * 10^{7} \mathrm{~cd} / \mathrm{m}^{2}$.

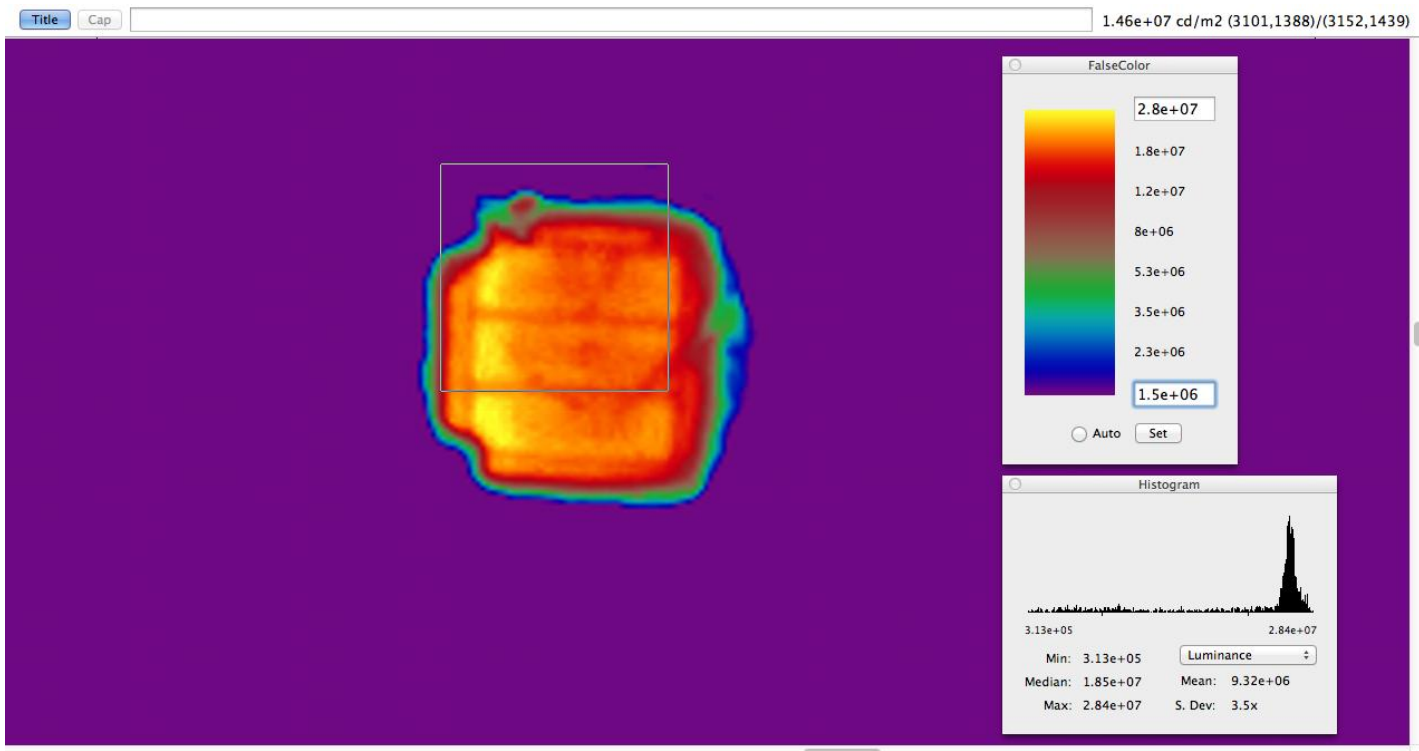

Figure 3-56A. Selected area (51x49 pixels) is moved around the LED chip. Mean value $9.32 * 10^{6} \mathrm{~cd} / \mathrm{m}^{2}$. 


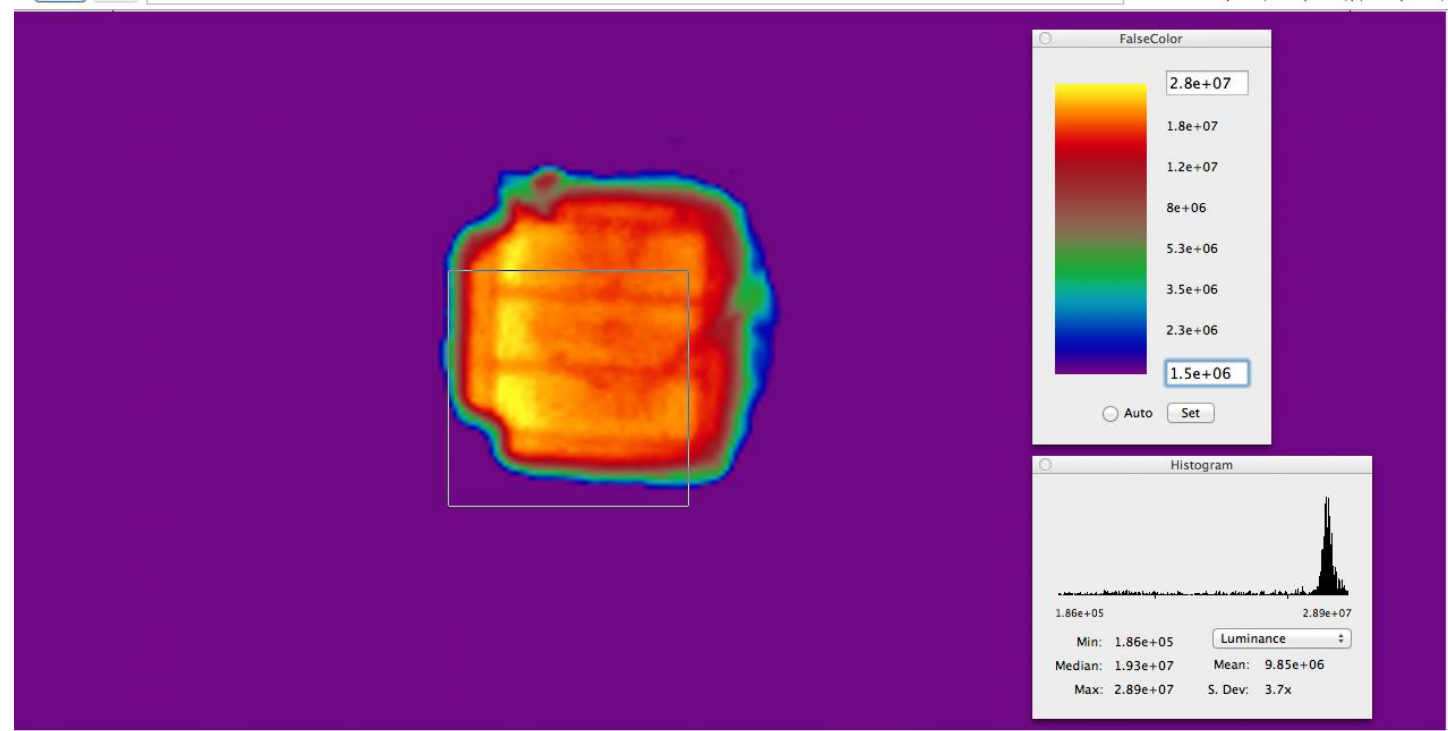

Figure 3-56B. Selected area ( $51 \times 49$ pixels) is moved around the LED chip. Mean value $9.85 * 10^{6} \mathrm{~cd} / \mathrm{m}^{2}$.

$1.25 \mathrm{e}+07 \mathrm{~cd} / \mathrm{m} 2(3122,1416) /(3174,1467)$

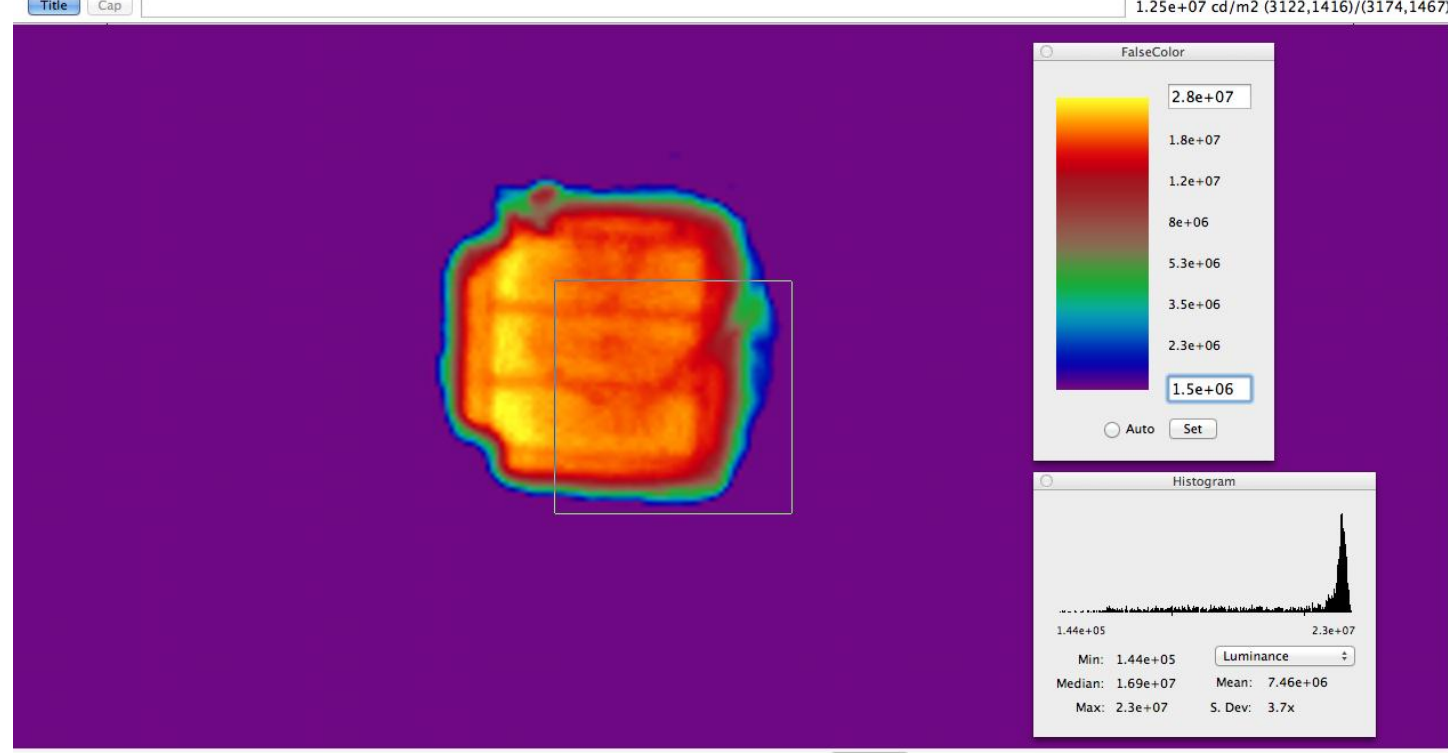

Figure 3-56C. Selected area (51x49 pixels) is moved around the LED chip. Mean value $7.46 * 10^{6} \mathrm{~cd} / \mathrm{m}^{2}$. 


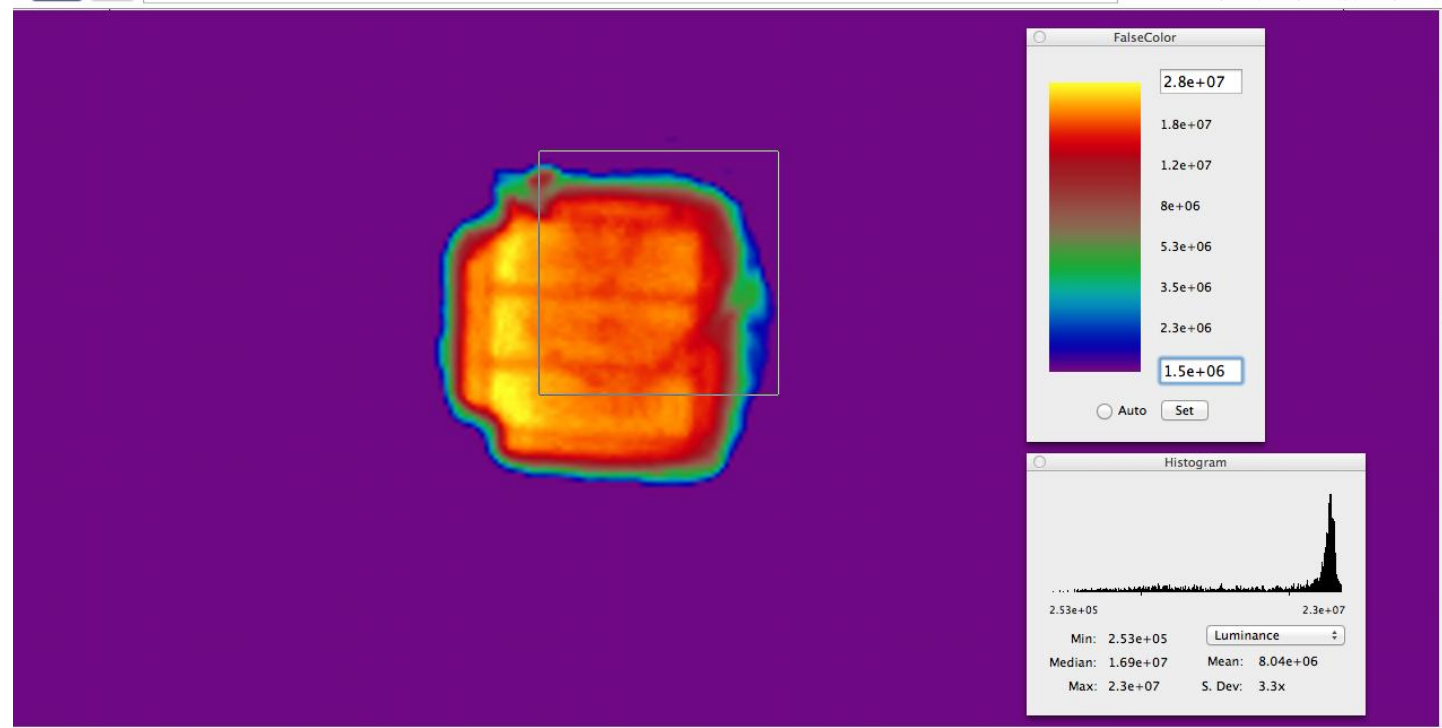

Figure 3-56D. Selected area (51x49 pixels) is moved around the LED chip. Mean value $8.04 * 10^{6} \mathrm{~cd} / \mathrm{m}^{2}$.

$1.38 \mathrm{e}+07 \mathrm{~cd} / \mathrm{m} 2(3093,1392) /(3146,1441)$

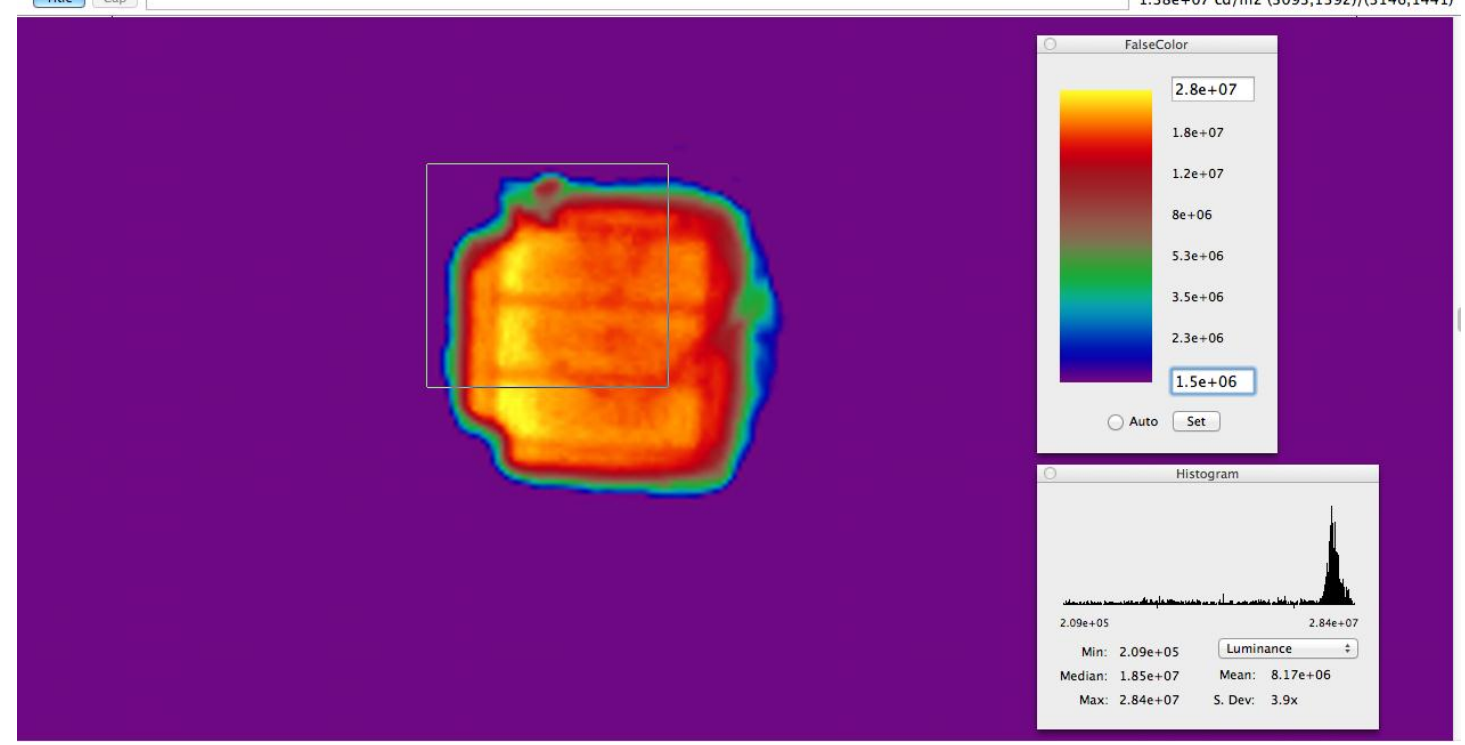

Figure 3-56E. Selected area (51x49 pixels) is moved around the LED chip. Mean value $8.17 * 10^{6} \mathrm{~cd} / \mathrm{m}^{2}$.

The next sequence (figures 3-57 A-D) shows the LED chip in pseudo colors with scale reduced in every subsequent image. It shows the non-uniformity of a single LED chip and LED's brightest parts. 


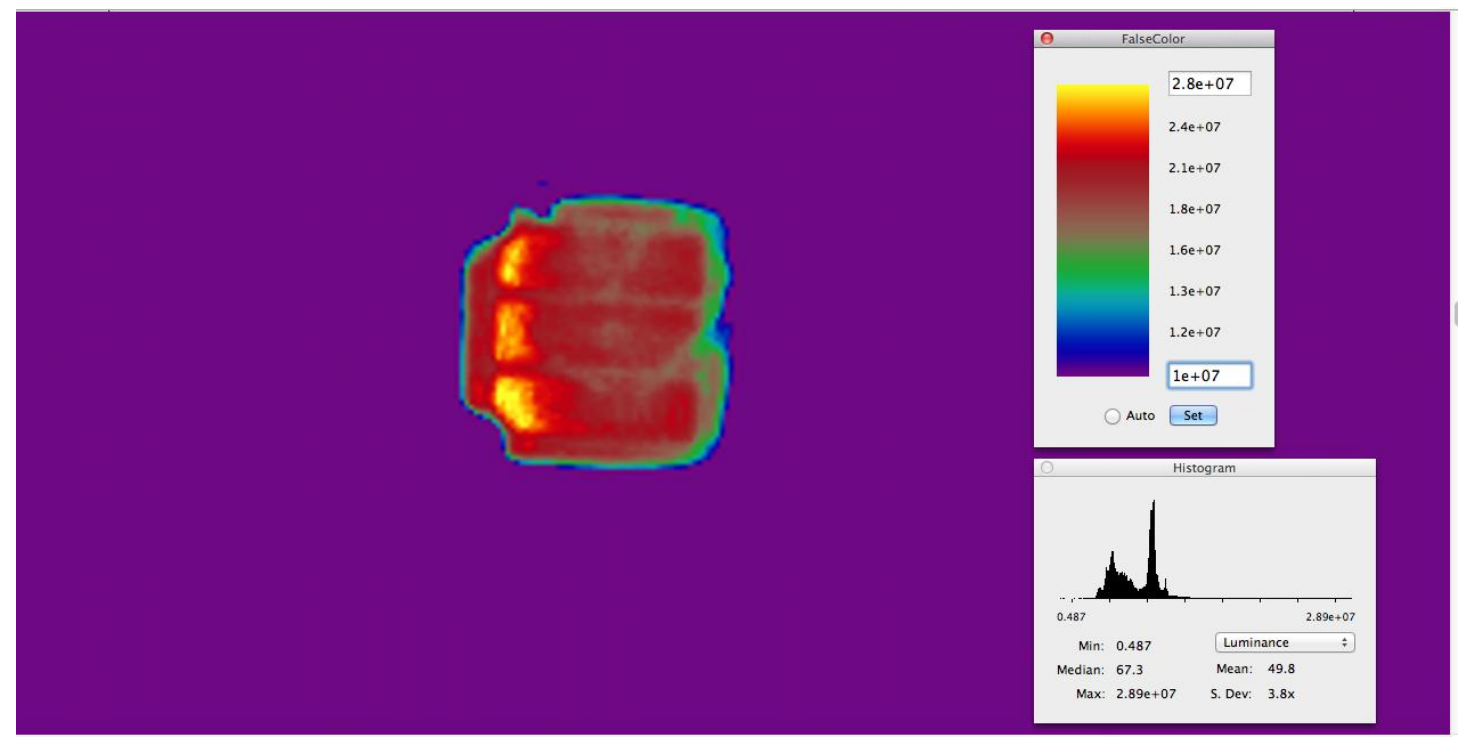

Figure 3-57A. The non-uniformity of a single LED chip

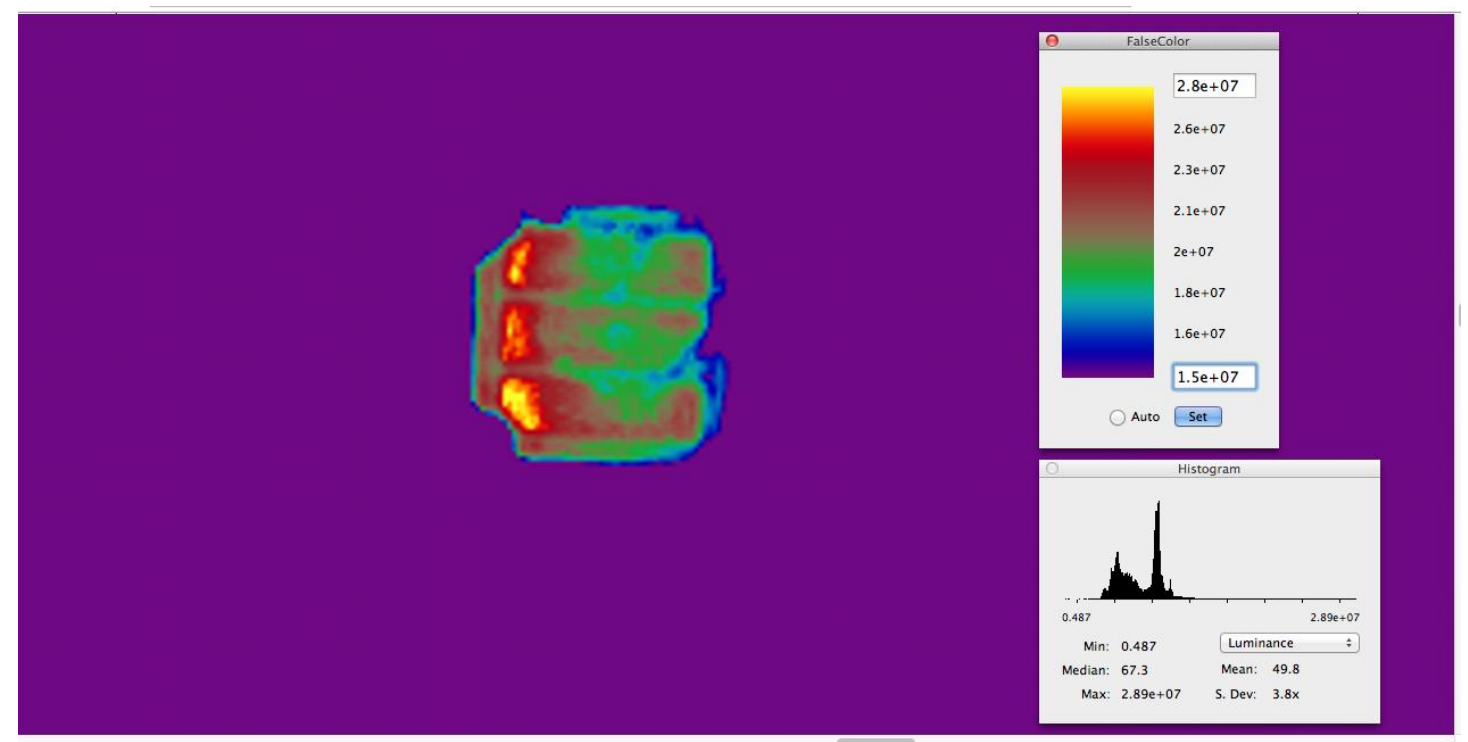

Figure 3-57B. The non-uniformity of a single LED chip 


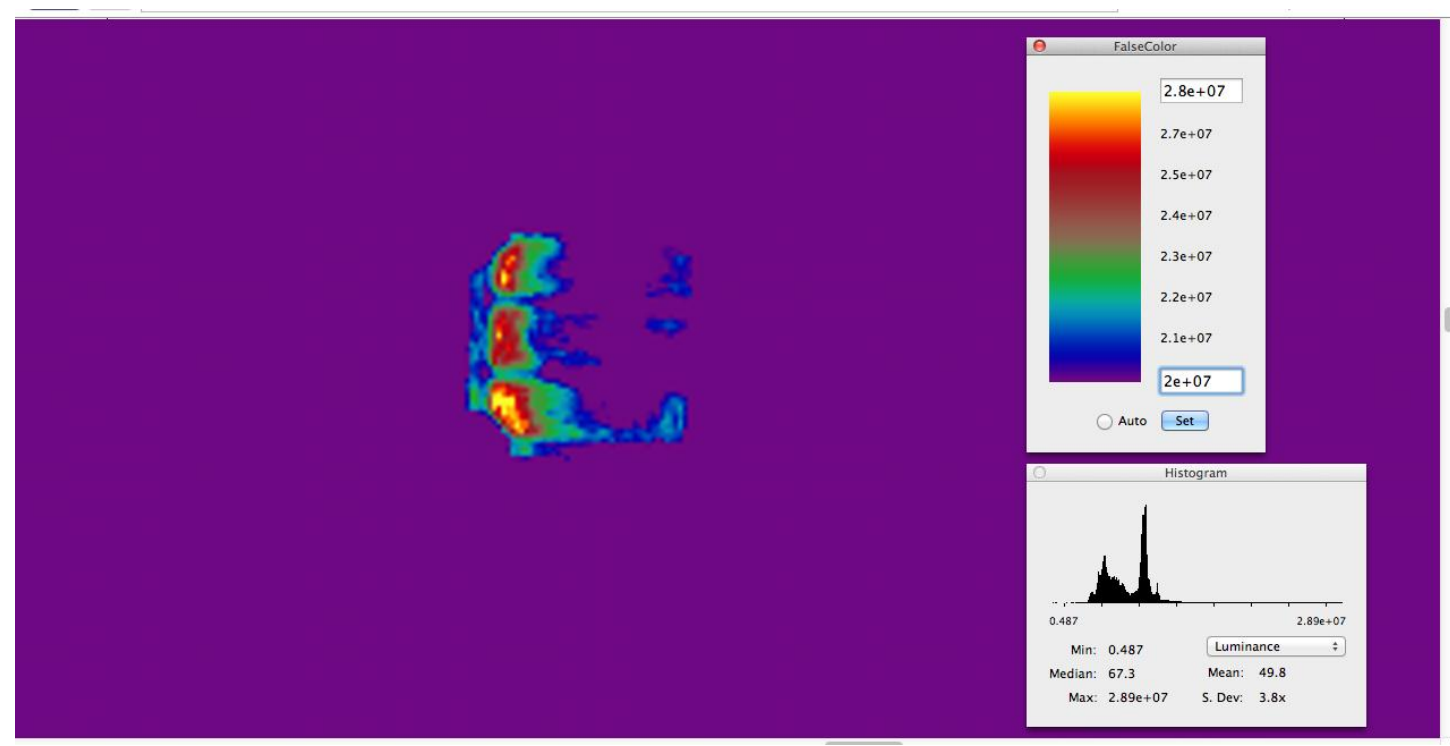

Figure 3-57C. The non-uniformity of a single LED chip

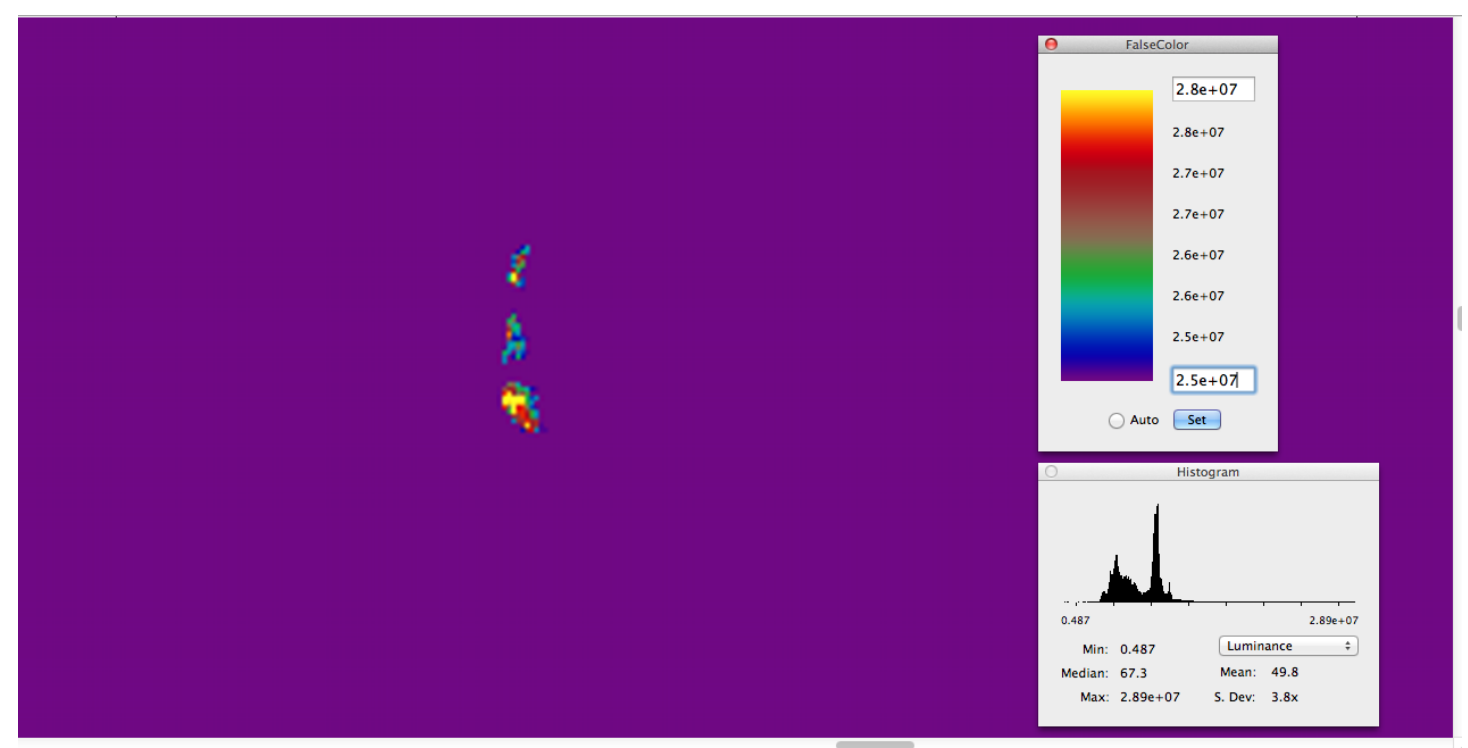

Figure 3-57D. The non-uniformity of a single LED chip 
The size of the LED chip in the image is $48 \mathrm{x} 48$ pixels (Photosphere) (figure 358). Obtained mean luminance value is $19.74 * 10^{6} \mathrm{~cd} / \mathrm{m}^{2}$.

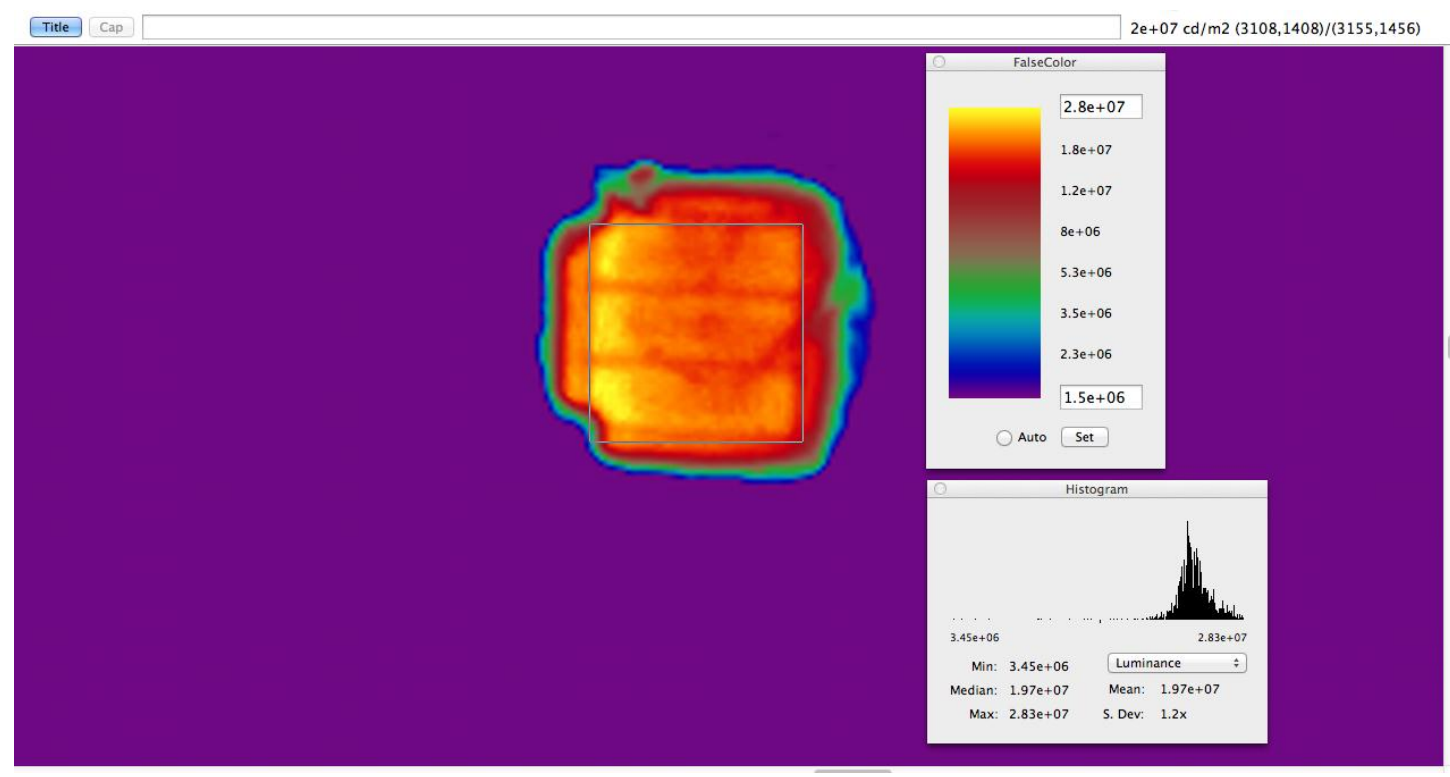

\section{Figure 3-58. Selected area of the LED chip (48x48 pixels) in pseudo colors}

Then image was cropped to obtain only a single LED chip in the image (figure 3-59).

The analysis of the HDR image was performed with the pvalue Radiance tool (appendix E). Pixel-by-pixel values were obtained with the following code in the terminal:

pvalue $-\mathrm{o}-\mathrm{pG}$ chipONLY.hdr

This will give 3 columns of data: $\mathrm{x}$ position, $\mathrm{y}$ position and green channel values. Green channel values have to be multiplied by *179 (see appendix D) in order to obtain luminance values. If only green channel values are needed without $\mathrm{x}, \mathrm{y}$ coordinates, the following has to be typed:

pvalue $-\mathrm{o}-\mathrm{pG}-\mathrm{d}$ chipONLY.hdr

And the maximum and minimum values can be found with pextrem tool (then multiplied by 179 as well): 


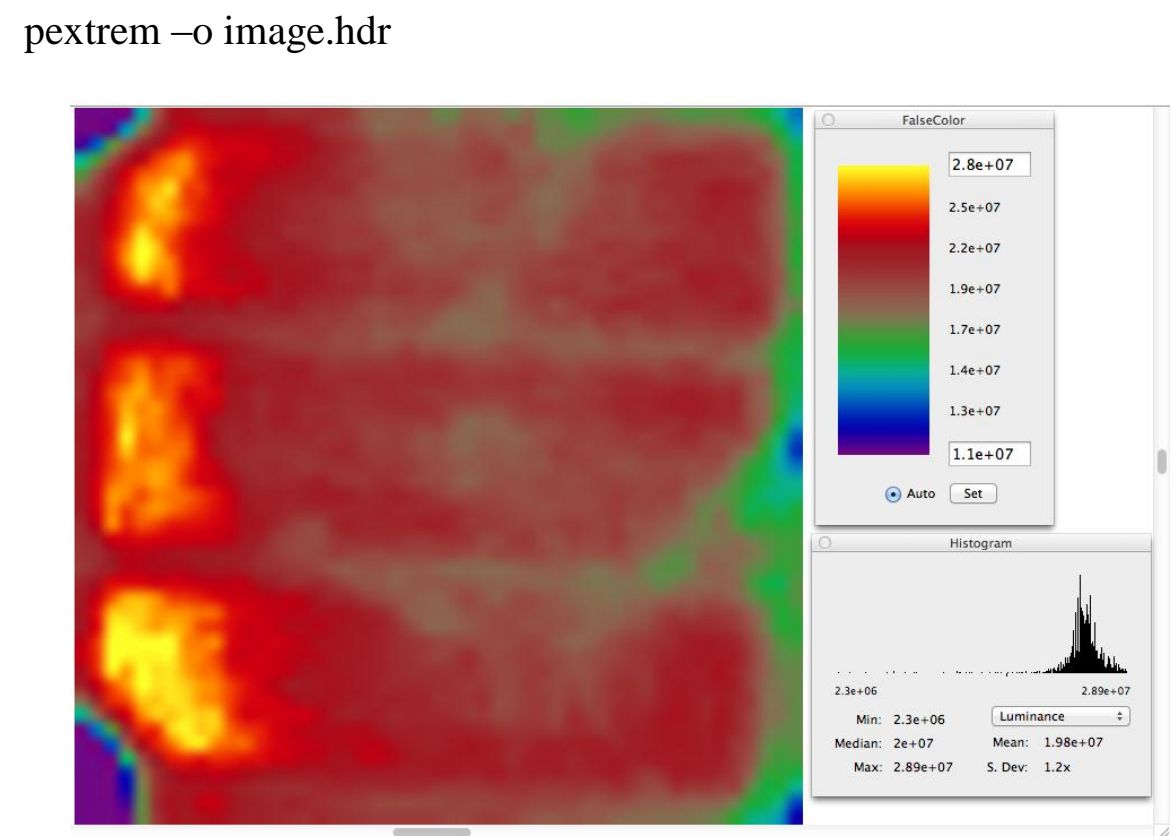

Figure 3-59. Cropped image of a single LED chip in pseudo colors (48 pixels by 48 pixels)

The data obtained with the pvalue Radiance tool was put onto the graph to see the distributions of luminance values along the $\mathrm{x}$ axis (figure 3-60, 3-61). 


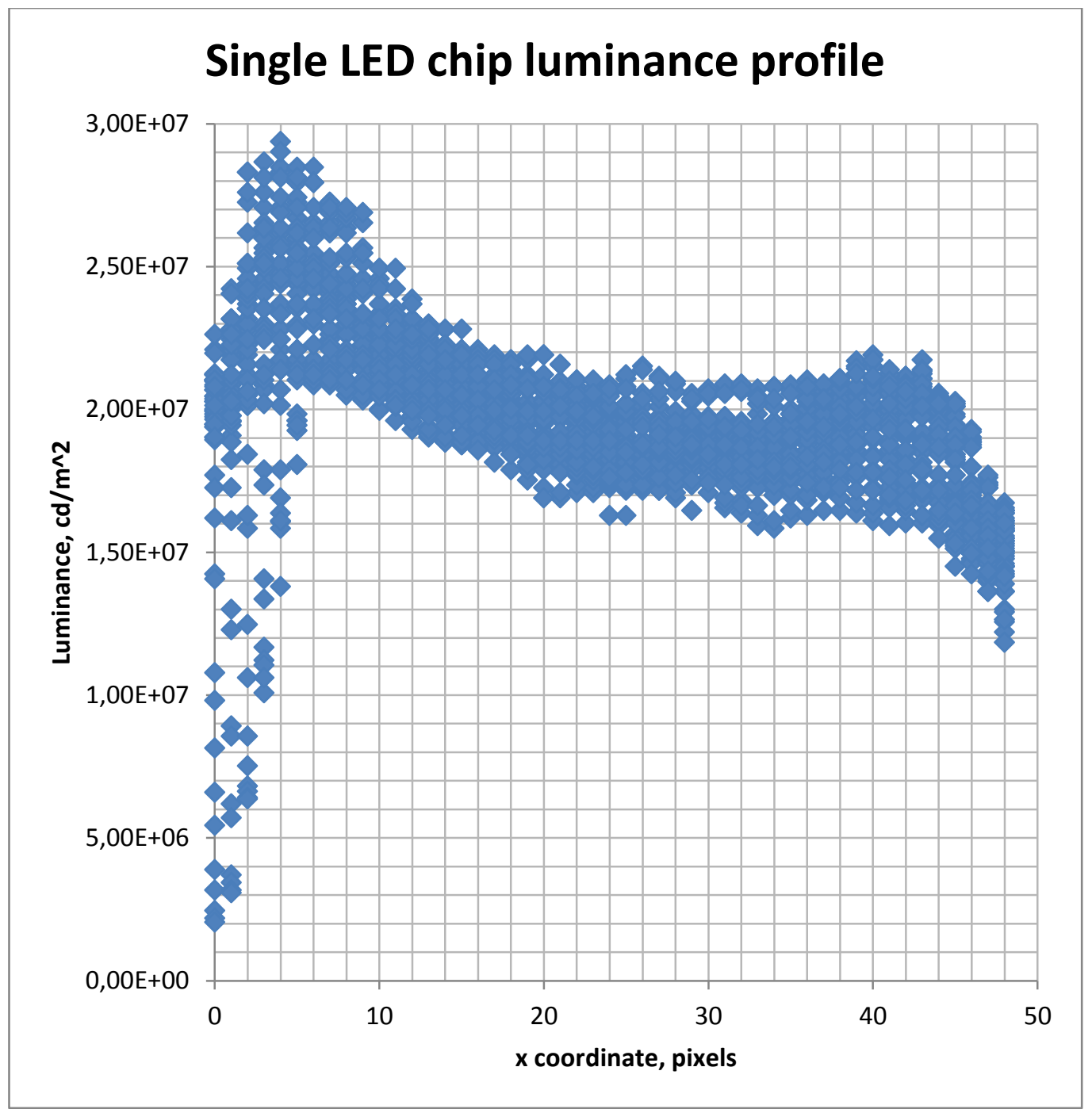

Figure 3-60. Luminance values obtained with the pvalue Radiance tool from a cropped HDRI of a single LED (48 pixels by 48 pixels)

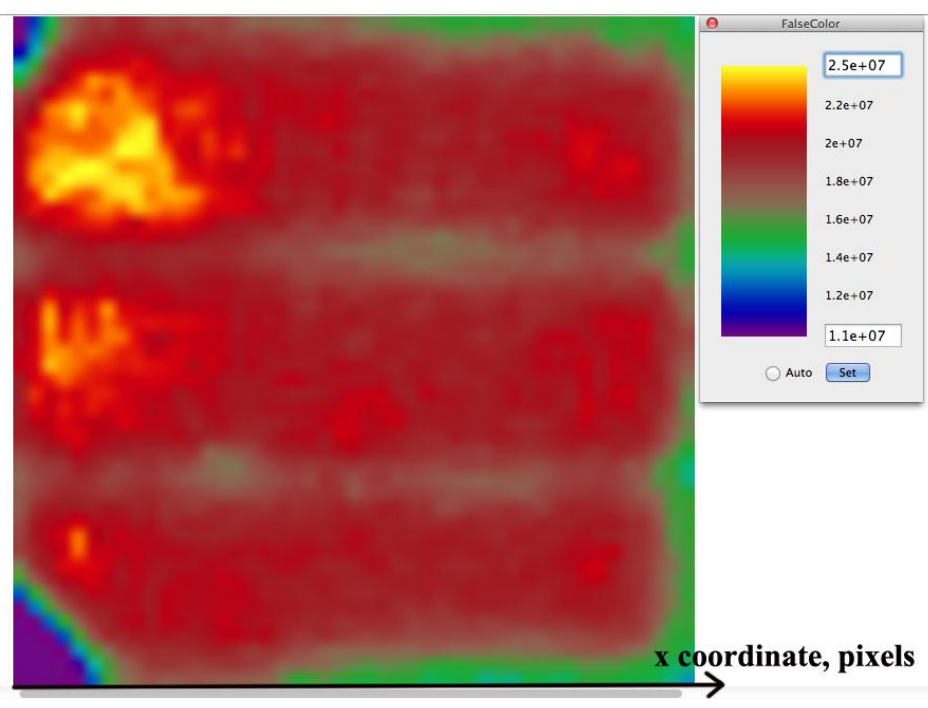

Figure 3-61. X coordinate on the cropped HDRI of a single LED (pseudo colors) 
Then luminance values were sorted in an ascending order in Excel. The data was described with the frequency distribution histogram (figure 3-62). Bins were made in $2 * 10^{6} \mathrm{~cd} / \mathrm{m}^{2}$ increments (table $3-15$ ); the number of pixels in every bin was calculated to obtain the frequency histogram.

Table 3-15. Bins of luminance values and number of pixels in every bin

\begin{tabular}{|c|c|}
\hline Bins & $\begin{array}{c}\text { Frequency } \\
\text { (number of } \\
\text { pixels in the } \\
\text { range) }\end{array}$ \\
\hline $1000000-3000000$ & 3 \\
\hline $3000000-5000000$ & 6 \\
\hline $5000000-7000000$ & 8 \\
\hline $7000000-9000000$ & 4 \\
\hline $9000000-11000000$ & 5 \\
\hline $11000000-13000000$ & 12 \\
\hline $13000000-15000000$ & 38 \\
\hline $15000000-17000000$ & 168 \\
\hline $17000000-19000000$ & 657 \\
\hline $19000000-21000000$ & 880 \\
\hline $21000000-23000000$ & 369 \\
\hline $23000000-25000000$ & 136 \\
\hline $25000000-27000000$ & 79 \\
\hline $27000000-29000000$ & 33 \\
\hline $29000000-31000000$ & 2 \\
\hline
\end{tabular}




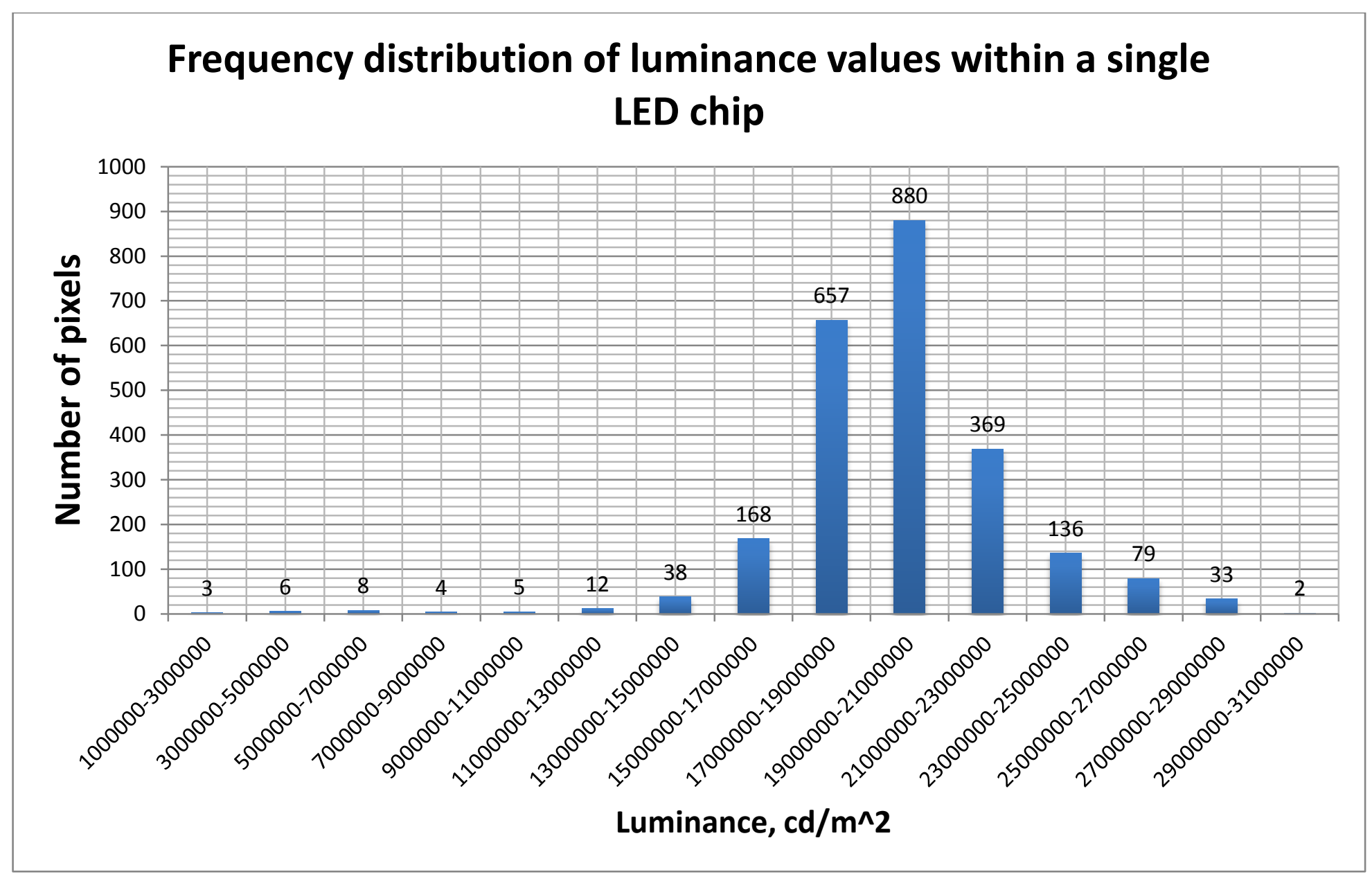

Figure 3-62. Frequency distribution of luminance values within a single LED chip obtained with the pvalue Radiance tool from a cropped HDRI 
Mean luminance for the LED chip obtained from the HDRI is $\mathbf{1 9 . 7 4 * 1 0 ^ { 6 }}$ $\mathrm{cd} / \mathrm{m}^{2}$

The error of the calculated average luminance value (section 3.2.3) compared to the mean luminance value of a single LED chip obtained from the HDRI was calculated:

$$
\begin{aligned}
& \text { Error }=\frac{\mid \text { calculated value }- \text { HDRI value } \mid}{\text { calculated value }} \times 100 \% \\
& \text { Error }=\frac{\left|19.44 * 10^{6}-19.74 * 10^{6}\right|}{19.44 * 10^{6}} \times 100 \%=1.5 \%
\end{aligned}
$$

Outcome: The HDRI captured a single LED (CANON EOS 7D fitted with CANON zoom lens $28-105 \mathrm{~mm}$ at $105 \mathrm{~mm}$, shutter speed $1 / 8000-1 / 15$ ' at the aperture size f/16, and 1/30-30', at the aperture size f/4.5; neutral density filter NDA2-703-002 with $\tau=0.0094$ was used, sequence was fused with raw2hdr) with an error of about $1.5 \%$ compared to the calculated value (obtained through illuminance measurements). Peak values in HDRI correspond to non-uniformity of a single LED chip. Low values in the luminance meter measurements indicate averaging areas of the LED luminance with its non-illuminating surroundings. 


\section{Chapter 4 Results and discussion}

This chapter discusses the challenges and results of current research and gives ideas for future work.

\subsection{Discussion of results and recommendations}

Challenges in measuring the luminance of a single LED chip are the following:

1. LED chip has a very small emitting surface (1.4 $\mathrm{mm}$ square side).

2. LED chip has a very high luminance (average luminance is in the range of $\left.(19.44-19.74) * 10^{6} \mathrm{~cd} / \mathrm{m}^{2}\right)$.

Two ways of measuring luminance with traditional methods were considered.

$\rightarrow \quad$ Measuring luminance of a single LED chip with a LS110 luminance meter even with a specific close-up lens (\#135) gives unreliable results due to inability of the meter's measuring area "fit" into the measured area of a light source (meter can measure a minimum diameter of $1.8 \mathrm{~mm}$ at minimum distance of 447 $\mathrm{mm})$.

If the luminance meter is aimed perfectly at the center of the LED chip the emitting chip covers only $77 \%$ of the LS110 measurement circle. If it is not aimed accurately, the average luminance will result in an even lower value since more of the non-illuminating area around the LED chip will be captured by a measuring circle of the luminance meter.

When measuring luminance of the LED chip, it exceeds the measuring range of the luminance meter (which is $999900 \mathrm{~cd} / \mathrm{m}^{2}$ for LS110). Thus, neutral density filter has to be implemented. For this task NDA2-703-002 with $\boldsymbol{\tau}=0.0094$ is used.

The highest obtained luminance is $\mathbf{6 . 9 7 * 1 0 ^ { 6 }} \mathrm{cd} / \mathrm{m}^{2}$. 
$\rightarrow \quad$ The second method of measuring LED luminance is through direct illuminance measurements in a constructed photometer. Luminances are calculated through obtained luminous intensity curve and projected area of a single LED chip. LED is a point light source which allows using inverse cosine square law. The assumption that LED chip is a uniform light source is made.

Luminous intensity curve for the measured LED chip has a cosine shape in polar coordinate system. The chip has same luminance values within the investigated angles ( -40 to 42.5 degrees) with $5 \%$ error.

The average luminance obtained from illuminance measurements in a created photometer and subsequent calculations is $19.44 * 10^{6} \mathrm{~cd} / \mathrm{m}^{2}$.

Such a drastic difference in calculated average luminance $\left(19.44 * 10^{6} \mathrm{~cd} / \mathrm{m}^{2}\right)$ of a single LED chip and value measured with the luminance meter $\left(6.97 * 10^{6} \mathrm{~cd} / \mathrm{m}^{2}\right)$ exists due to aiming problems. It is confirmed with the HDRI experiments.

$\rightarrow \quad$ Vignetting effect of the camera/lens combination was tested with uniform luminance source. The non-uniformity of ULS is less than $1 \%$.

The difference between the errors of luminance values due to optical vignetting effect for two obtained images with peripheral illumination correction on and off is about $9 \%$. Peripheral illumination correction does improve the quality of HDRI, but not as it is necessary for the photometric measurements. Initially camera is not developed for luminance measurements, so manufacturer wouldn't account for the accurate photometric measurements. Visually one can't see light fall off towards the edges on the photograph. Image sequence used for creating an HDRI is taken with enabled peripheral illumination correction in the camera.

Vignetting effect for EOS7D fitted with CANON zoom lens 16-35 is reduced when fully zoomed in (at $35 \mathrm{~mm}$ focal length). 
Vignetting effect depending on different variables should be thoroughly tested to accumulate more statistical data. This can be a separate study.

$\rightarrow \quad$ Digital images undergo many transformations during their lifetime. RAW files bypass the problems of correction manipulations entirely because the response is linear. Working with RAW images is a reasonable way to get around most of the in-camera processing that undermines absolute CIE color accuracy.

For the same aperture size vignetting effect is smaller for the HDRI created with RAW images compared to the fused with JPEG images (at the aperture size f/4.5 at $105 \mathrm{~mm}$ focal length vignetting effect for the HDRI fused with JPEG 20.0\%, with RAW $15.3 \%)$.

$\rightarrow \quad$ HDRI's ability to capture an incandescent light source ( RAW images are fused with raw2hdr) compared to the luminance meter measurement is within 1.4 $\%$ error at $28 \mathrm{~mm}$ and $105 \mathrm{~mm}$ focal lengths. The results give confidence in using RAW images for the HDRI creation, calibrating and analyzing an image in Photosphere afterwards.

$\rightarrow \quad$ If only one or few pixels have certain value in a range of $(0,255), \mathrm{RGB}$ histograms in Photoshop CS5 show it clearly. Thus, it allows determining if the sequence used for making the HDRI is captured properly. While CANON Digital Photo Professional software shows histograms of photos, if only few pixels correspond to a specific value of brightness, it cannot be seen on histogram, which can lead to photometric mistake. Only general shape of exposures can be seen. Photosphere doesn't allow viewing histogram for a single JPEG photo. It shows statistics for only an HDR image obtained from a sequence.

$\rightarrow \quad$ Flare is an issue when a bright light source is captured. When calibrating an image, for the correct results calibration should be done in the area 
where lens flare doesn't visually affect the image. The red area on the figure 4-1 is the area of the reflectance standard that is affected by the flare. If the image is calibrated in that area it would lead to a photometric mistake.

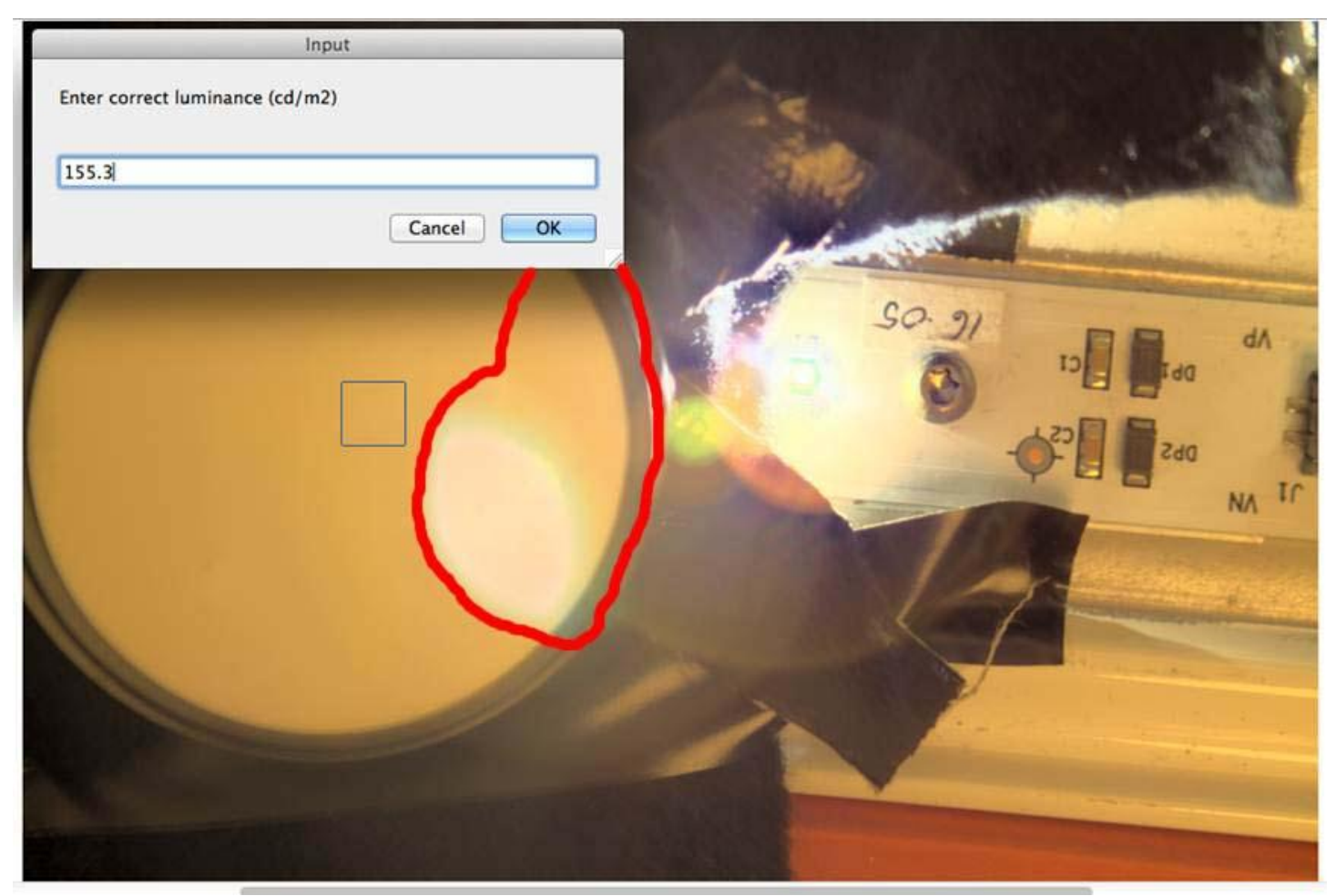

Figure 4-1. Calibrating an image in the zone where flare doesn't affect the image

$\rightarrow \quad$ LED is captured with CANON EOS 7D fitted with 28-105 mm at 105

$\mathrm{mm}$ focal length. If to use only one aperture size (for e.g. f/16) while capturing the LED chip on the long exposure end (so that there are no RGB values below 20) with a ND filter, exposure time has to be too long (more than 5 minutes for one photo). This is unacceptable for taking images of fast-changing lighting environments (for e.g. the sun and sky).

Two aperture sizes are used (f/4.5 and f/16). Vignetting effect depends on aperture size, so it has to be checked. For the purpose of this research optical vignetting effect is not taking into consideration since all the HDRI measurements are 
taken in the parts of the image, where luminance error due to vignetting effect is less than $5 \%$.

Shutter speeds used are 1/8000-1/15' at the aperture size f/16, and 1/30-30', at the aperture size f/4.5. Neutral density filter NDA2-703-002 with $\tau=0.0094$ is used. Camera is normal to the LED. Image sequence is fused in raw2hdr and calibrated in Photosphere at white reflectance standard $(\rho=99 \%)=155.3 \mathrm{~cd} / \mathrm{m}^{2}$.

Depending on the area within which the measurement is averaged in Photosphere, the readings change dramatically.

Mean value obtained for the selected area smaller than the LED chip is 20.4*10 $\mathbf{1 0}^{\mathbf{6}} \mathrm{cd} / \mathrm{m}^{2}$. The size of the LED chip in the image is 48 pixels $\mathrm{x} 48$ pixels (Photosphere). The area that LS110 luminance meter measures is $23 \%$ more than the size of the LED chip (section 3.2.2). It corresponds to a size of about 53 pixels x 53 pixels in the image.

Aiming at the LED with the luminance meter is very difficult. The inability of luminance meter to "fit" into measuring area of the LED gives unreliable results. It is confirmed by luminance values obtained from the HDRI when selected area (around 53 pixels by 53 pixels) is moved around the LED chip. It measures LED with the borders outside of the chip. The range of mean values is $\left(\mathbf{7 . 4 6 - 9 . 8 5 )} * \mathbf{1 0} 0^{\mathbf{6}} \mathrm{cd} / \mathrm{m}^{2}\right.$.

Obtained mean luminance value for the LED chip in Photosphere is $\mathbf{1 9 . 7 4 * 1 0 ^ { 6 }}$ $\mathrm{cd} / \mathrm{m}^{2}$

The error of the calculated average luminance compared to the mean luminance of a single LED chip from the HDRI (equation 3.12):

$$
\begin{aligned}
& \text { Error }=\frac{\mid \text { calculated value }-H D R I \text { value } \mid}{\text { calculated value }} \times 100 \% \\
& \text { Error }=\frac{|19442757-19735285|}{19442757} \times 100 \%=1.5 \%
\end{aligned}
$$


The summary of luminance measurements of a single LED chip is showed in table 4-1.

Table 4-1. Luminance values obtained with traditional methods and acquired with the HDRI (RAW images are fused in raw2hdr).

\begin{tabular}{|c|c|c|c|}
\hline & $\begin{array}{c}\text { HDRI luminance, } \\
\mathrm{cd} / \mathrm{m}^{2}\end{array}$ & $\begin{array}{c}\text { Measurements, } \\
\mathrm{cd} / \mathrm{m}^{2}\end{array}$ & Error, \% \\
\hline $\begin{array}{l}\text { Area smaller than a } \\
\text { single LED chip }\end{array}$ & $20.4 * 10^{6}$ & - & - \\
\hline \multirow{4}{*}{$\begin{array}{l}\text { Area of a single } \\
\text { LED chip } \\
\text { (48 pixels } \times 48 \\
\text { pixels) } \\
(1.4 \mathrm{~mm} \times 1.4 \mathrm{~mm})\end{array}$} & $19.74 * 10^{6}$ & \multirow{4}{*}{$\begin{array}{c}\mathbf{1 9 . 4 4} * 10^{6} \\
\text { (Illuminance } \\
\text { meter and } \\
\text { calculations) }\end{array}$} & \multirow[t]{4}{*}{1.5} \\
\hline & $\begin{array}{c}\text { Standard Deviation (SD) } \\
2.92 * 10^{6}\end{array}$ & & \\
\hline & $\begin{array}{c}\text { 1.96SD } \\
5.73 * 10^{6}\end{array}$ & & \\
\hline & $\begin{array}{c}95 \% \text { confidence } \\
\text { interval } \\
(19.74+0.57) * 10^{6}\end{array}$ & & \\
\hline $\begin{array}{l}\text { Area } 23 \% \text { bigger } \\
\text { than a single LED } \\
\text { chip } \\
\text { (53 pixels x } 53 \\
\text { pixels) }\end{array}$ & $\begin{array}{c}(7.46-9.85)^{*} 10^{6} \\
\text { Average 8.58*10 }\end{array}$ & $\begin{array}{c}\mathbf{6 . 9 7} * \mathbf{1 0} \\
\text { (Luminance } \\
\text { meter) }\end{array}$ & 19 \\
\hline
\end{tabular}

HDRI captured a single LED (CANON EOS 7D fitted with CANON zoom lens $28-105 \mathrm{~mm}$ at $105 \mathrm{~mm}$, shutter speed $1 / 8000-1 / 15$ ' at the aperture size $\mathrm{f} / 16$, and $1 / 30-30$ ' at the aperture size $\mathrm{f} / 4.5$; neutral density filter NDA2-703-002 with $\tau=0.0094$ and fused with raw $2 \mathrm{hdr}$ ) with an error of about $1.5 \%$ compared to the calculated average luminance (obtained through illuminance measurements) (figure 42). 
Frequency distribution of luminances within a single LED

chip

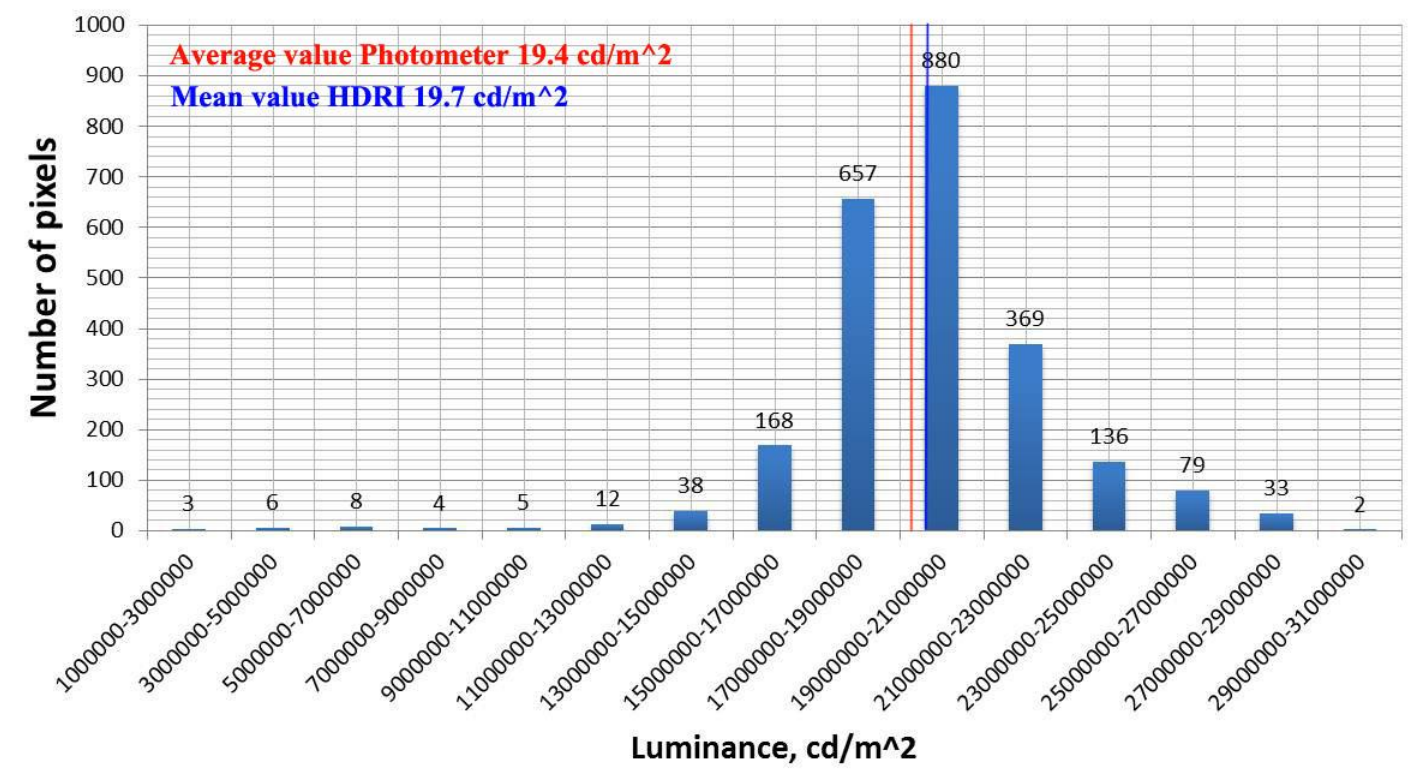

Figure 4-2. Frequency distribution of luminances within a single LED chip obtained from the HDRI; mean luminance from the HDRI; and average value from illuminance measurements/calculations

The illuminance (T10) and luminance (LS110) meters give average

measurements. The luminance meter gives an average within the measuring circle, and the illuminance meter averages the signal over the whole sensor area of the receiver, while the HDRI has a capability of high resolution information from many pixels within the image. Peak values in HDRI correspond to non-uniformity of a single LED chip. Since the smallest measuring size of the luminance meter measures LED chip with non-illuminating surroundings, it cannot "catch" non-uniformities with higher values that are smaller than the measuring area. The result is low luminance.

The HDRI technique shows positive results in capturing a bright light source with a narrow light distribution (a single LED chip). It gives confidence in using the technology in lighting research and to expand its capabilities. Although many challenges exist, they can be address in future work. 


\subsection{Future research}

$\rightarrow \quad$ For the future research more measurements of LED chips under a variety of experimental settings should be conducted to acquire more statistical data on HDRI's ability to accurately capture photometric data.

$\rightarrow \quad$ Measuring the whole luminaire that consists of arrays with multiple LEDs is an interesting area to investigate. Flare might play a critical role in making an HDR image of the LED fixture because of the multiple ghost images. Also, vignetting filter has to be applied, since big light fall off is observed towards the edge of the image.

$\rightarrow \quad$ In the application with LED luminaires installed, camera zoom settings might have a significant impact on results of luminance measurements.

$\rightarrow \quad$ More detailed research on vignetting effect for different software, aperture sizes and other variables should be performed in a future work. Vignetting experiments with peripheral correction on/off fused in different software might be a part of it.

$\rightarrow \quad$ The spectral luminous efficiency function underestimates perceived LEDs' intensity of wavelengths toward the blue end of the spectrum. It might be investigated while obtaining luminance values with the HDRI. 


\section{References}

Anaokar, Smita, and Moeck, Martin. "Validation of high dynamic range imaging to luminance measurement”. LEUKOS - Journal of Illuminating Engineering Society of North America (2005, (2)): 133-44.

"Anyhere Software", accessed September, 2011, http://anyhere.com/.

Banterle, Francesco, Debattista, Kurt, Artusi, Alessandro, Pattanaik, Sumanta, Myszkowski, Karol, Ledda, Patrick, and Chalmers, Alan. "High dynamic range imaging and low dynamic range expansion for generating HDR content". Computer Graphics Forum 28 (2009, (8)): 2343-67.

Bellia, Laura, Cesarano, Arcangelo, Fabio Iuliano, Giuseppe, and Spada, Gennaro. "HDR luminance mapping analysis system for visual comfort evaluation" (paper presented at 2009 IEEE Intrumentation and Measurement Technology Conference, I2MTC 2009, May 5, 2009 - May 7, 2009).

Busch, David D. David busch's canon EOS 5D mark II guide to digital SLR photography. (Boston, Mass.: Course Technology 2011).

Cai, H., and Chung, T. M. "Improving the quality of high dynamic range images”. Lighting Research \& Technology (2011, 43 (1), (03)): 87-102.

“CANON”, accessed February, 2012. http://www.canon.com/.

"Cambridge in Color website. A learning community for photographers", accessed March, 2012, http://www.cambridgeincolour.com/tutorials/. 
Chung, Tse-Ming, and T. H. Ng, Roger. "Variation of calibration factor over time for high dynamic range photography in a single daylit interior scene". Journal of Light and Visual Environment (2010, 34 (2)): 87-93.

[CIE] Commission Internationale de l'Eclairage. 2007. 127-2007 Technical Report. "Measurement of LEDs", Vienna, CIE, 2007, accessed March, 2012, http://www.scribd.com/doc/56472704/CIE-127-2007-Measurement-of-LEDs-SecondEdition.

[CIE] Commission Internationale de l'Eclairage. 2007. 177-2007 Technical Report. “Color Rendering of White LED Light Sources”, Vienna, CIE, 2007.

“CREE. The LED lighting”, accessed February, 2012, http://www.cree.com/, http://www.cree.com/products/xlamp_xpe.asp.

Debevec, Paul E., and Malik, Jitendra. "Recovering high dynamic range radiance maps from photographs" (paper presented at Proceedings of the 1997 Conference on Computer Graphics, SIGGRAPH, August 3, 1997 - August 8, 1997).

DiLaura, David, Houser, Kevin, Mistrick, Richard, and Steffy, Gary, 2011. The Lighting Handbook, $10^{\text {th }}$ edition. Reference and application. (New York: Illuminating Engineering Society 2011).

Eble-Hankins ML, Waters CE. "The UNL Glare Apparatus”. LEUKOS Journal of Illuminating Engineering Society of North America (2009, 6(1)): 79-90.

“EXIF", accessed January, 2012, http://exif.org/.

“General Electric Lighting”, accessed March, 2012, http://www.gelighting.com/na/. 
“GetData Graph Digitizier”, accessed March, 2011, http://www.getdata-graphdigitizer.com/.

Guglielmetti, Robert, and Scheib, Jennifer. "High Dynamic Range Imaging for Glare Analysis" (presented at the annual architectural and commercial trade lighting show LightFair in Philadelphia, May 16, 2011).

“HDRI mailing list", accessed March, 2012, http://www.radianceonline.org/mailman/listinfo/hdri.

Herwig, Johannes, and Pauli, Josef. "Recovery of the response curve of a digital imaging process by data-centric regularization" (paper presented at 4th International Conference on Computer Vision Theory and Applications, VISAPP 2009, February 5, 2009 - February 8, 2009).

[IES] Illuminating Engineering Society. 2010. G-2-10. "Guideline for the Application of General Illumination ("White") LED Technologies".

[IES] Illuminating Engineering Society. 2005. TM-16-05. “Technical Memorandum Light Emitting Diode (LED) Sources and Systems by Illuminating Engineering Society”.

[IES] Illuminating Engineering Society. 2011. TM-21-11. “Projecting Long Term Lumen Maintenance of LED Light Sources by Illuminating Engineering Society".

[IES] Illuminating Engineering Society. 2008. LM-79-08. “Approved Method: Electrical and Photometric Testing of Solid-State Lighting Devices by Illuminating Engineering Society". 
[IES] Illuminating Engineering Society. 2008. LM-80-08. “Approved Method: Measuring Lumen Depreciation of LED Light Sources by Illuminating Engineering Society".

Inanici, Mehlika. "Evaluation of high dynamic range photography as a luminance data acquisition system”. Lighting Research and Technology (2006, 38 (2)): 123-36.

Inanici, Mehlika. "Evaluation of high dynamic range image-based sky models in lighting simulation". LEUKOS - Journal of Illuminating Engineering Society of North America (2010, 7 (2)): 69-84.

Inanici, Mehlika , and Navvab, Mojtaba. “ The virtual lighting laboratory: Perpixel luminance data analysis". LEUKOS - Journal of Illuminating Engineering Society of North America (2009, 3 (2)): 89-104.

"International Commission on Illumination (CIE)", accessed March, 2012, http://cie.co.at/; http://www.cie.co.at/Technical+Committees; http://div2.cie.co.at/.

Jacobs, A. "High dynamic range imaging and its application in building research”. Advances in Building Energy Research (2007, (1)): 177-202.

Kim, Seon Joo, and Pollefeys, M. "Robust radiometric calibration and vignetting correction". Pattern Analysis and Machine Intelligence, IEEE Transactions on $(2008,30(4)): 562-76$.

Kohtaro, Kohmoto, Fukashi, Ogata, Fusao, Hoshino and Weidong, Qian. "Luminance measurement of HP LEDS and photobiological safety risk evaluation of 
them" (paper presented at CIE Light and Lighting Conference with Special Emphasis on LEDs and Solid State Lighting, Budapest, Hungary, May $27-29^{\text {th }}, 2009$ ).

“Konica Minolta", accessed January, 2012, http://www.konicaminolta.com/.

Konis, K., Lee, E. S., and , R. D. "Visual comfort analysis of innovative interior and exterior shading systems for commercial buildings using high resolution luminance images". LEUKOS - Journal of Illuminating Engineering Society of North America (2011, 7 (3)): 167-88.

"Labsphere", accessed March, 2012, http://www.labsphere.com/products/uniform-light-source-calibrationsystems/default.aspx

Ledda, Patrick, Chalmers, Alan, and Seetzen, Helge. " HDR displays: A validation against reality" (paper presented at 2004 IEEE International Conference on Systems, Man and Cybernetics, SMC 2004, October 10, 2004 - October 13, 2004).

Mitsunaga, Tomoo, and Nayar, Shree K. "Radiometric self calibration". Proceedings of the IEEE Computer Society Conference on Computer Vision and Pattern Recognition (1999, 1): 374-80.

Moeck, Martin. “Accuracy of luminance maps obtained from high dynamic range images". LEUKOS - Journal of Illuminating Engineering Society of North America (2007, 4 (2)): 99-112.

Moeck, Martin, and Anaokar, Smita. “ Illuminance analysis from high dynamic range images". LEUKOS - Journal of Illuminating Engineering Society of North America (2006, 2 (3)): 211-28. 
Ohno, Yoshi. "Overview of measurement standards for solid state lighting”, accessed February, 2012, http://www.cormusa.org/uploads/1_CORM_Workshop_YO.pdf.

“Introduction to Computer Vision”, accessed October, 2011, https://www.cs.drexel.edu/ kon/introcompvis/lectures/wIntroCompVis_W9.pdf.

"Photo reports on cameras and lenses", accessed January, 2012, http://www.dpreview.com/, http://www.the-digital-picture.com/, http://photozone.de/.

"Radiant Imaging website" accessed October, 2011, http://www.radiantimaging.com/.

Rea, M. S., and Jeffrey, I. G. "New luminance and image analysis system for lighting and vision I. equipment and calibration". Journal of the Illuminating Engineering Society (1990, 19 (1)): 64-72.

Reinhard, Erik, Heidrich, Wolfgang, Debevec, Paul, Pattanaik, Sumanta, Ward, Greg, Myszkowski, Karol . High Dynamic Range Imaging, Second Edition: Acquisition, Display, and Image-Based Lighting. Second Edition. (Burlington, MA: Elsevier Inc., 2010).

Robertson, Mark A., Borman, Sean, and Stevenson, Robert L. "Estimationtheoretic approach to dynamic range enhancement using multiple exposures". Journal of Electronic Imaging (2003, 12 (2)): 219-28.

Stumpfel, Jessi, Jones, Andrew, Wenger, Andreas, Tchou, Chris, Hawkins, Tim, and Debevec, Paul. " Direct hdr capture of the sun and sky”. Submitted to Afrigraph 2004. 
“U.S. Department of Energy", accessed February, 2012, http://www1.eere.energy.gov/buildings/ssl/index.html.

Van, Den Wymelenberg, Inanici, Mehlika, and Johnson, Peter. "The effect of luminance distribution patterns on occupant preference in a daylit office environment". LEUKOS - Journal of Illuminating Engineering Society of North America (2010, 7 (2)): 103-22.

Ward, Greg, and Simmons, Maryann. "JPEG-HDR: A backwards-compatible, high dynamic range extension to JPEG” (paper presented at STOC'05: Proceedings of the 37th Annual ACM Symposium on Theory of Computing, May 22, 2005 - May 24, 2005).

"WebHDR", accessed October, 2011, http://www.jaloxa.eu/webhdr/calibrate.shtml .

Weinert, Jonathan. LED Lighting Explained. (Philips Color Kinetics Inc.: 2010).

Xiao, Feng, DiCarlo, Jeffrey M., Catrysse, Peter B., and Wandell, Brian A. "High dynamic range imaging of natural scenes" (paper presented at Final Program and Proceedings of the 10th IS and T/SID Color Imaging Conference: Color Science, Systems and Applications, November 12, - November 15, 2002). 


\section{Appendix A: Table of experimental settings from studies of HDRI in lighting}

Table A. Experimental settings from studies of HDRI validation in lighting research

\begin{tabular}{|c|c|c|c|c|c|c|c|c|}
\hline $\begin{array}{l}\text { Features } \\
\text { /Settings } \\
\text { in papers }\end{array}$ & (Inanici 2006) & $\begin{array}{c}\text { (Anaokar et al. } \\
\text { 2005) }\end{array}$ & $\begin{array}{c}\text { (Moeck et al. } \\
\text { 2006) }\end{array}$ & (Moeck 2007) & $\begin{array}{c}\text { (Chung et al. } \\
\text { 2010) }\end{array}$ & $\begin{array}{c}\text { (Cai et al. } \\
\text { 2011) }\end{array}$ & $\begin{array}{l}\text { (Stumpfel } \\
\text { et al. } \\
\text { 2004) }\end{array}$ & (Inanici 2010) \\
\hline Camera & $\begin{array}{c}\text { Nikon Coolpix } \\
5400 \text { digital }\end{array}$ & $\begin{array}{l}\text { Nikon Coolpix 5400, } \\
\text { digital camera with a } \\
5.2 \text { megapixel CCD } \\
\text { array }\end{array}$ & $\begin{array}{l}\text { Nikon Coolpix } \\
5400 ; \\
\text { Olympus C-5060 } \\
\text { Wide Zoom built } \\
\text { in } 2003 \text { (for } \\
\text { building) }\end{array}$ & $\begin{array}{c}\text { Canon Eos } 350 \\
\text { D Digital Rebel } \\
\text { XT, digital SLR } \\
\text { camera with } 8 \\
\text { megapixels }\end{array}$ & Canon EOS 350D & $\begin{array}{c}\text { Canon EOS } \\
\text { 350D }\end{array}$ & $\begin{array}{c}\text { Canon } \\
\text { EOS-1Ds }\end{array}$ & $\begin{array}{c}\text { Canon EOS } \\
\text { 5D }\end{array}$ \\
\hline Lens & $\begin{array}{c}\text { Fisheye Nikon } \\
\text { FC-E9 }\end{array}$ & $\begin{array}{c}\text { F2.8-F4.6 } 4 \mathrm{X} \text { optical } \\
\text { zoom lens. Focal } \\
\text { range of } 5.8-24 \\
\text { mm, which is } \\
\text { equivalent to } 28 \text { - } \\
116 \mathrm{~mm} \text { for } 35 \mathrm{~mm} \\
\text { film }\end{array}$ & $\begin{array}{c}\mathrm{f} 2.8 \text { - f/8.0 zoom } \\
\text { lens (for } \\
\text { building) }\end{array}$ & Canon zoom lens & $\begin{array}{c}\text { Ultra wide angle } \\
\text { lens Sigma 10-20 } \\
\text { mm F4-5.6 EX DC } \\
\text { HSM human vision }\end{array}$ & $\begin{array}{l}\text { Ultra wide angle } \\
\text { lens Sigma 10- } \\
20 \mathrm{~mm} \mathrm{F4-5.6} \\
\text { EX DC HSM } \\
\text { human vision }\end{array}$ & $\begin{array}{c}8 \mathrm{~mm} \\
\text { Sigma } \\
\text { fisheye } \\
\text { lens }\end{array}$ & $\begin{array}{l}\text { Sigma } 8 \mathrm{~mm} \\
\text { F3.5 EXDG }\end{array}$ \\
\hline $\begin{array}{l}\text { Focal } \\
\text { length }\end{array}$ & $5.6 \mathrm{~mm}$ & - & $24 \mathrm{~mm}$ & & $10 \mathrm{~mm}$ & $10,14,20 \mathrm{~mm}$ & & \\
\hline $\begin{array}{l}\text { Angle of } \\
\text { view }\end{array}$ & $190^{\circ}$ & - & - & - & $96^{\circ}$ horizontal view & - & & $\begin{array}{l}180^{\circ} \text { angle of } \\
\text { view and equi- } \\
\text { angle } \\
\text { projection } \\
\text { properties }\end{array}$ \\
\hline $\begin{array}{c}\text { White } \\
\text { balance }\end{array}$ & Daylight & $\begin{array}{c}\text { Preset. Matrix Auto } \\
\text { White Balance with } \\
\text { TTL control }\end{array}$ & $\begin{array}{c}\text { Automatic white } \\
\text { balance }\end{array}$ & - & Daylight & Fluorescent & & Daylight \\
\hline $\begin{array}{c}\text { Auto- } \\
\text { bracketing }\end{array}$ & Off & - & - & - & $\begin{array}{c}\text { On, Automatic } \\
\text { Exposure } \\
\text { Bracketing mode - } \\
\text { 4EV to 4EV of the } \\
\text { metered exposure }\end{array}$ & $\begin{array}{l}\text { On, } 4 \text { ranges of } \\
\text { exposure values }\end{array}$ & $\begin{array}{l}\text { Special } \\
\text { program }\end{array}$ & \\
\hline Image size & $2592 \times 1944$ & - & $2592 \times 1944$ & JPEG 3456 by & Large/fine & Large/fine & & \\
\hline
\end{tabular}




\begin{tabular}{|c|c|c|c|c|c|c|c|c|}
\hline & pixels & & $\begin{array}{c}\text { pixels. HDRI } \\
1296 \times 972\end{array}$ & 2304 pixels & & $\begin{array}{c}\text { (JPEG) } \\
\left(3456 \_2304\right. \\
\text { pixels, PPI 72) }\end{array}$ & & \\
\hline Sensitivity & ISO 100 & - & - & ISO 100 & ISO 100 & ISO 100 & & ISO100 \\
\hline $\begin{array}{c}\text { Exposure } \\
\text { mode }\end{array}$ & $\begin{array}{c}\text { Manual exposure } \\
\text { mode }\end{array}$ & Aperture priority & - & Aperture priority & Aperture priority & - & & $\begin{array}{c}\text { Manual } \\
\text { exposure mode }\end{array}$ \\
\hline $\begin{array}{c}\text { Description } \\
\text { of settings } \\
\text { in exposure } \\
\text { mode }\end{array}$ & $\begin{array}{c}\text { Aperture is fixed } \\
\text { f/4.0. } \\
\text { Shutter speeds } \\
\text { 2s to } 1 / 4000 \mathrm{~s} \text {. }\end{array}$ & $\begin{array}{c}\text { F-stop } 7.9 \text { (no zoom) } \\
\text { Shutter speed of the } \\
\text { camera was changed } \\
\text { in one step } \\
\text { increments } 2 \text { s to } \\
1 / 4000 \text { s }\end{array}$ & $\begin{array}{c}\text { Building. F-stop } \\
\text { is } \mathrm{f}-2.8 \\
\text { The shutter } \\
\text { speeds are } 1 / 5, \\
1 / 8,1 / 10,1 / 15 \\
1 / 20, \text { and } \\
1 / 30 .\end{array}$ & $\begin{array}{c}\text { f-stop 5.6, } \\
\text { shutter speeds } \\
1 / 4000,1 / 2500, \\
1 / 1250,1 / 800, \\
1 / 400 \text {, and } 1 / 200\end{array}$ & $\begin{array}{l}\text { Aperture is fixed } \\
\text { f/8.0. } \\
\text { Shutter speeds } 2 \mathrm{~s} \\
\text { to } 1 / 4000 \mathrm{~s} \text {. } \\
\text { AF one shot, AE } \\
\text { lock, exposure } \\
\text { compensation none }\end{array}$ & $\begin{array}{c}\text { Six apertures } \\
\text { were } \\
\text { investigated f/4 } \\
\text { (at fj10mm } \\
\text { only), f/5.6, f/8, } \\
\text { f/11, f/16, f/22, } \\
\text { AF one shot, } \\
\text { AE lock, } \\
\text { exposure } \\
\text { compensation } \\
\text { none, Shutter } \\
\text { speed with in } \\
\text { sec, with } 1 \mathrm{EV} \\
\text { step } 30,15,8,4, \\
2,1,0.5,1 / 4, \\
1 / 8,1 / 15,1 / 30, \\
1 / 60, \\
1 / 125,1 / 250, \\
1 / 500,1 / 1000, \\
1 / 2000,1 / 4000\end{array}$ & $\begin{array}{c}\text { F4, f } 16 \\
\text { Aperture } \\
\text { Shutter } \\
\text { speed of 1- } \\
\text { 1/8000", } \\
3.0 \text { neutral } \\
\text { density } \\
\text { filter }\end{array}$ & $\begin{array}{l}\text { F5.6 for the } \\
\text { sky, f4 shutter } \\
\text { speeds } 15 \mathrm{~s} \text { to } \\
1 / 30 \text { and f16 } \\
\text { for the sun } \\
\text { shutter speeds } \\
1 / 15-1 / 8000 \text {. } \\
\text { Neutral density } \\
\text { filter Kodak } \\
\text { Wratten } 2 \\
\text { Optical Filter }\end{array}$ \\
\hline $\begin{array}{l}\text { Software } \\
\text { used for } \\
\text { creation } \\
\text { HDRI }\end{array}$ & Photosphere & - & Photosphere & - & - & Photosphere & & Photosphere \\
\hline $\begin{array}{l}\text { Scene for } \\
\text { analysis }\end{array}$ & $\begin{array}{l}\text { Black painted } \\
\text { room without } \\
\text { daylight to office } \\
\text { spaces and } \\
\text { outdoors }\end{array}$ & $\begin{array}{l}\text { For dependence of } \\
\text { error on color - } \\
\text { Color Munsell } \\
\text { Chips. }\end{array}$ & - & Targets outdoors & $\begin{array}{l}\text { Interior scene with } \\
\text { very dark, very } \\
\text { bright, large neutral } \\
\text { grey and white } \\
\text { surfaces with }\end{array}$ & $\begin{array}{c}\text { Target indoors } \\
\text { with fluorescent } \\
\text { lighting }\end{array}$ & Outdoors & Outdoors \\
\hline
\end{tabular}




\begin{tabular}{|c|c|c|c|c|c|c|c|c|}
\hline & & & & & $\begin{array}{l}\text { smooth gradients. } \\
\text { Color targets } \\
\text { indoor with } \\
\text { daylight without } \\
\text { direct sun }\end{array}$ & & & \\
\hline $\begin{array}{c}\text { Light } \\
\text { sources }\end{array}$ & $\begin{array}{l}\text { Incandescent, } \\
\text { fluorescent, } \\
\text { metal halide }\end{array}$ & $\begin{array}{c}\text { Fluorescent } \\
\text { CCT }=4100 \mathrm{~K} \\
\text { CRI }=80 \\
\text { Metal Halide } \\
\text { Sylvania } \\
\text { MP70/U/Med } \\
\text { CCT }=3000 \mathrm{~K} \\
\text { CRI }=75 \\
\text { Mercury Vapor GE } \\
\text { H250WDX 37-5 } \\
\text { CCT }=4000 ; \text { CRI }=45\end{array}$ & - & $\begin{array}{l}\text { Sunny day, } \\
\text { outdoors }\end{array}$ & $\begin{array}{l}\text { Stable overcast sky } \\
\text { conditions }\end{array}$ & $\begin{array}{c}\text { Fluorescent, low } \\
\text { and high } \\
\text { ambient light } \\
\text { level }\end{array}$ & $\begin{array}{l}\text { Daylight } \\
\text { and the } \\
\text { sun }\end{array}$ & $\begin{array}{l}\text { Daylight and } \\
\text { the sun }\end{array}$ \\
\hline Targets & $\begin{array}{c}\text { Grey targets } \\
(\rho=4-87 \%) \text {, grey } \\
28 \% \text { with white } \\
\text { surrounding } 87 \% \text {, } \\
\text { and black } 4 \% \text {, } \\
\text { white-then- } \\
\text { black, black- } \\
\text { then-white. The } \\
\text { Macbeth } \\
\text { ColorChecker. }\end{array}$ & $\begin{array}{l}\text { Grey targets, color } \\
\text { Munsell chips }\end{array}$ & $\begin{array}{l}\text { Twelve sized } \\
\text { patches on a } \\
\text { white paper for } \\
\text { assessment of E; } \\
\text { Application on } \\
\text { architectural } \\
\text { elements of a } \\
\text { building }\end{array}$ & $\begin{array}{c}\text { Matt grey cards, } \\
140 \text { color } \\
\text { checkers }\end{array}$ & $\begin{array}{l}24 \text { colors X-Rite } \\
\text { Color Checker }\end{array}$ & $\begin{array}{c}54 \text { grey, } 18 \\
\text { colored targets, } \\
\text { light source }\end{array}$ & $\begin{array}{l}\text { The sun } \\
\text { and sky }\end{array}$ & $\begin{array}{c}\text { The sun and } \\
\text { sky }\end{array}$ \\
\hline
\end{tabular}




\section{Appendix B: Specification of equipment}

\section{Cameras:}

CANON EOS 7D

CANON Rebel T1i

\section{Lenses:}

Canon EF 28-105mm f/3.5-4.5 II USM Lens (according to Photo reports websites as of October 2011)

Horizontal viewing angle $65^{\circ}-19^{\circ} 20^{\prime}$

Diagonal viewing angle $75^{\circ}-23^{\circ} 30^{\prime}$

Canon EF 16-35mm f/2.8L II USM

\section{LED luminaire:}

GE lighting. Evolve ${ }^{\mathrm{TM}}$ LED Garage Light - Medium Square

\section{EGMS-0-WL-N-60-P-C-10-white}

$0=120-277$

WL=wide;

$\mathrm{N}=\mathrm{N} / \mathrm{A}$ distribution orientation;

$60=$ LED color temperature $6000 \mathrm{~K}$;

$\mathrm{P}=$ Polycarbonate;

$\mathrm{C}=$ Clear with Frosted Portion;

$10=$ Surface Mounted;

White $=$ Color .

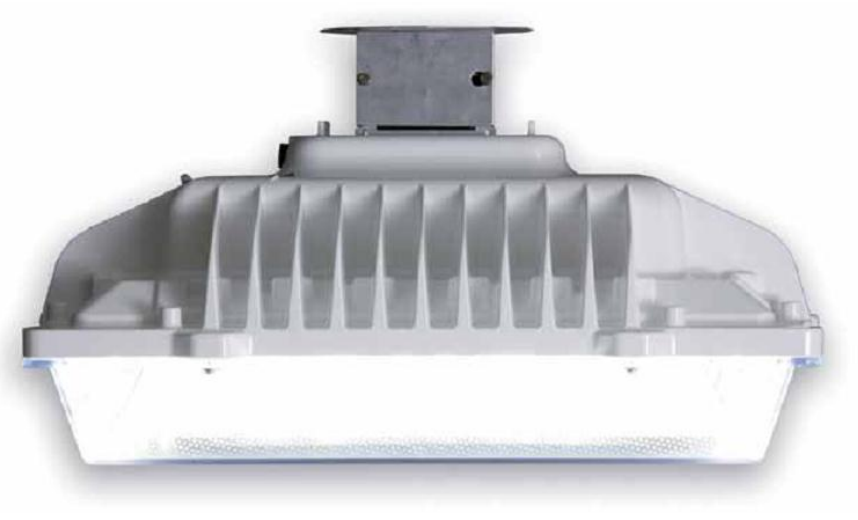




\section{Product Features}

High quality lighting meets long life and controllability with the Evolve ${ }^{T M}$ LED Garage Light. This fixture features an advanced LED optic specifically designed for applications such as parking decks with low mounting heights to provide high uniformity and excellent vertical light distribution with reduced glare and effective security light levels. It enables the instant-on capabilities needed to make the most of parking areas. There is also a unique option for up-light versions, not found in other LED garage fixtures. This provides a safe, driver friendly installation, eliminating the "cave effect" that often occurs without up-light.

The Evolve LED Garage Light significantly reduces energy and maintenance expenses over the life of the system and is also RoHS compliant, giving you a lead and mercury free lighting solution.

\section{Applications}

- Garage, warehouse, walkway and stairway lighting using a high efficiency LED optical system providing high uniformity and excellent vertical light distribution with reduced glare and effective security light levels.

\section{Housing}

- Die-cast aluminum housing with integral cast heat sinks to maximize heat transfer for electrical components and provide long LED life.

\section{LED \& Optical Assembly}

- LED optic specifically designed for applications such as parking decks with low mounting heights.

- Utilizes high brightness LEDs, $70 \mathrm{CRI}$ at $6000 \mathrm{~K}$ typical.

- LM-79 tests and reports are performed in accordance with IESNA standards.

\section{Lumen Maintenance}

- System rating is 50,000 hours at $L 85$. Contact factory for $L$ rating (Lumen Depreciation) beyond 50,000 hours.

\section{Ratings}

- UL/CUL listed, suitable for damp locations. Wet location listing available with select mounting options.

- IP 65 rated optical enclosure per ANSI C136.25-2009.

- Temperature rated at $-40^{\circ}$ to $40^{\circ} \mathrm{C}$ ambient.

- RoHS compliant, contains no lead or mercury.

\section{Mounting}

- Available with nine different mounting options providing a versatile product to meet almost any field installation condition.

\section{Finish}

- Corrosion resistant polyester powder painted minimum 2.0 mil, thickness.

- Applied inside and out of the housing for corrosion protection.

- Standard colors: Gray \& White.

- RAL and custom colors available.

\section{Electrical}

- 120-277 volt and 347-480 volt available.

- System power factor is $>90 \%$ and THD $<20 \%$.

- Class "A" sound rating.

- Integral surge protection non-dimming: - For 120-277VAC per IEEE/ANSI C62.41.-1991, 4kV/2kA Location Category B2 (120 Events)

- For 347-480VAC per IEEE/ANSI C62.41.-1991, 6kV/3kA Location Category B3 (120 Events)

- Integral surge protection GE dimming: - For 120-480VAC per IEEE/ANSI C62.41.2-2002. 6kV/3kA Location Category B (120 Events)

- Optional high capability surge protection per IEEE/ANS C62.41.2-2002.

- Rating 1 - 10kV/5kA Location Category (120 events) - Rating 2 - 6kV/3 kA Location Category C-Low 15000 events)

- EMI: Title 47 CFR Part 15 Class A

Warranty

- 5-year limited system warranty standard. 
Ordering Number Logic

Medium Square (EGMS)

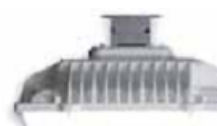

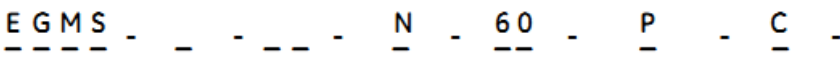

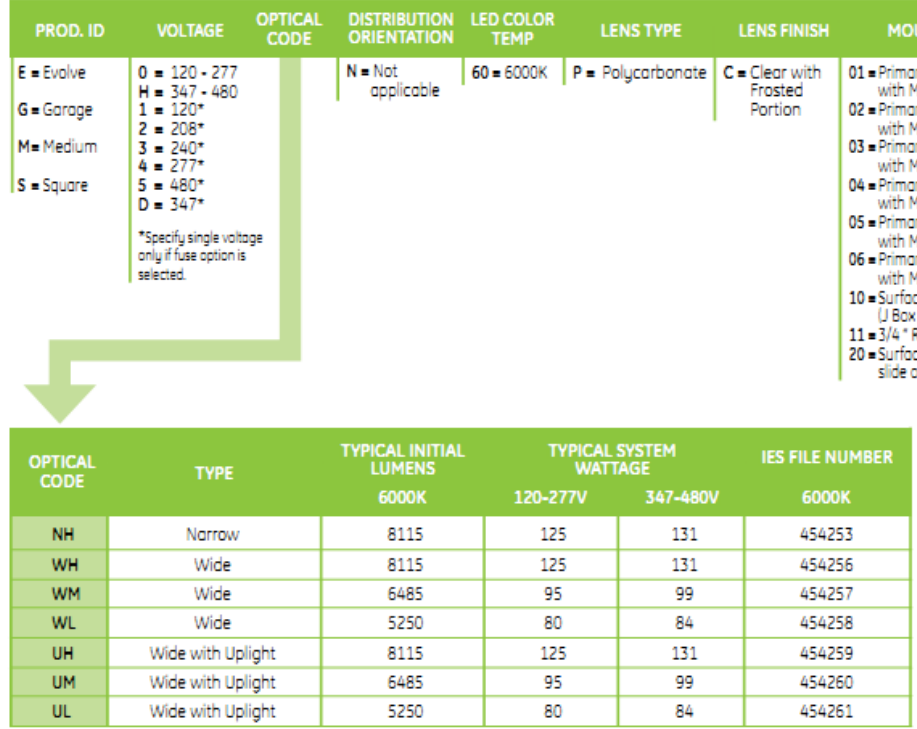

\section{Photometrics}

EGMS Type V - Narrow Square (NH) 8,115 Lumens, 6000K (GE454253.ies)

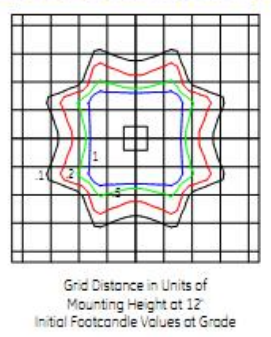

Light Distribution - No Uplight

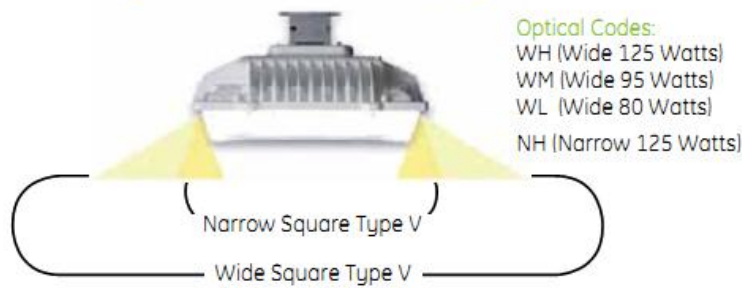

EGMS Type V - Wide Square (WH) EGMS Type V - Wide Square with Uplight (UH) 8,115 Lumens, 6000K (GE454256.ies)

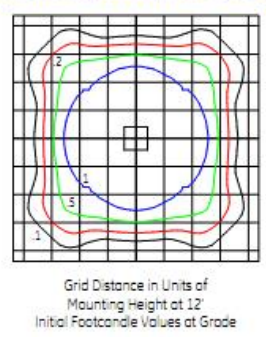

8,115 Lumens, 6000K [GE454259.ies)

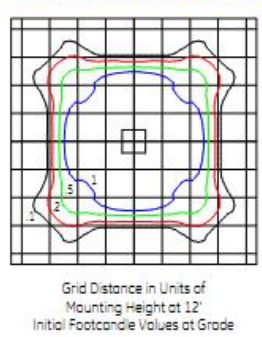

Light Distribution - With Uplight

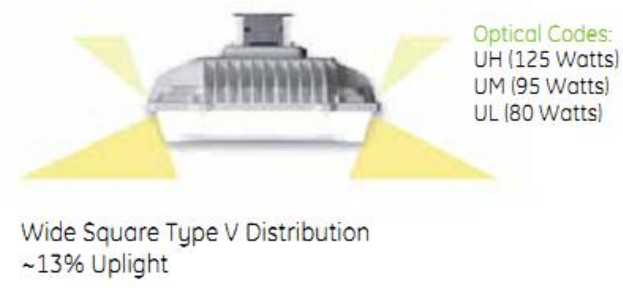




\section{Product Dimensions}

Surface Mounting with Slide on Wiring Box J-Box Not Included

Surface Mounting to J-Box Not Included

Sliding Disconnect Mounting

Pendant Mounting

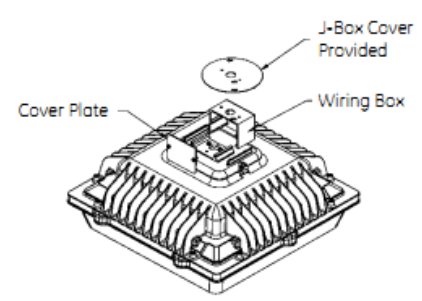

Mounting Option 20 shown above

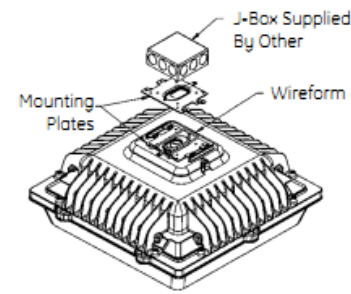

Mounting Option 10 shown above

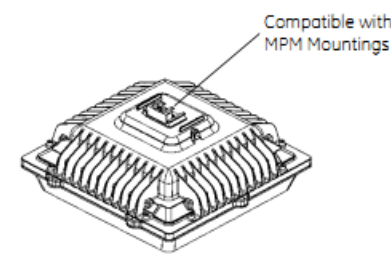

For Mounting Options $01-06$

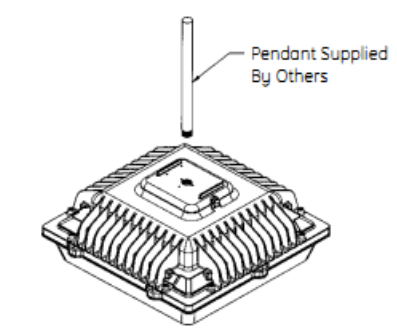

Mounting Option 11 shown above
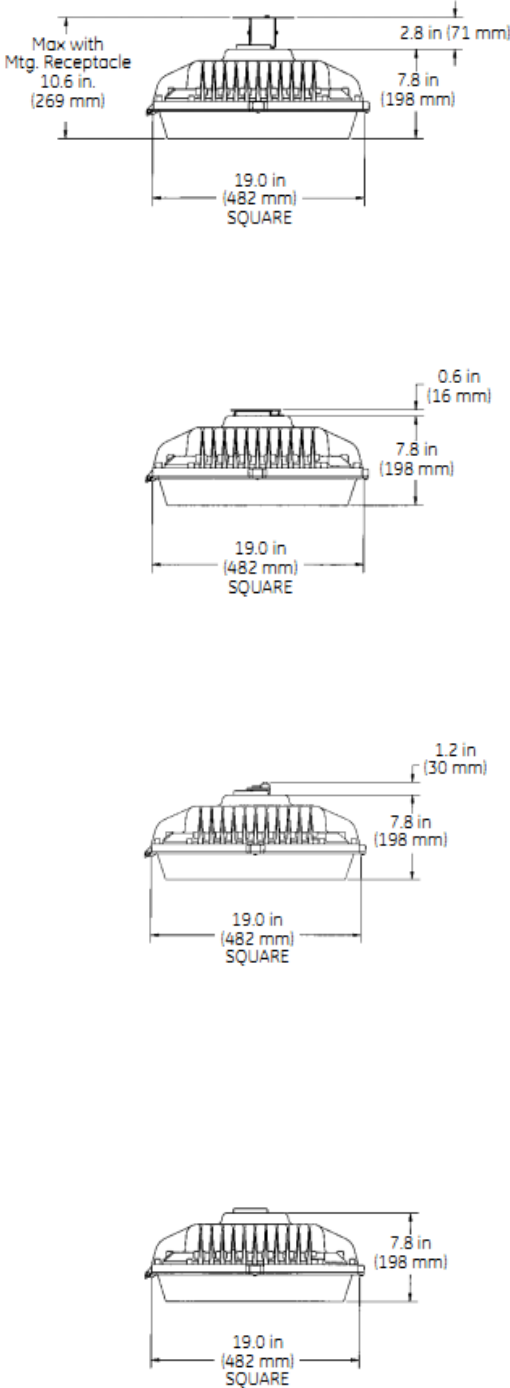

GE Lighting Solutions, LLC is o subsidiary of the General Eectric Company. Evave ond the GE brand and logo ore trodemarks of the General Electric Company.
Q 2011 GE Lighting Solutions, LLC. Informotion provided is subject to change without notice. All val Les ore design or typical values when measured under loboratory conotions.

OLP.2851/Rev. 03/25/111

\section{LEDs used in the luminaire:}

CREE LED (CREE LED lighting website)

First LED in the array was measured (Cool white 6000K). 


\section{Cree $^{\circledR}$ XLamp $^{\circledR}$ XP-E LEDs}

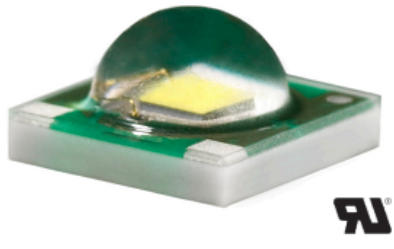

PRODUCT DESCRIPTION

The XLamp XP-E LED combines the proven lighting-class performance and reliability of the XLamp XR-E LED in a package with $80 \%$ smaller footprint. The XLamp XP-E LED continues Cree's history of innovation in LEDs for lighting applications with wide viewing angle, symmetrical package, unlimited floor life and electrically neutral thermal path.

Cree XLamp LEDs bring high performance and quality of light to a wide range of lighting applications, including color-changing, portable and personal, outdoor, indoor-directional, transportation, stage and studio, commercial and emergency-vehicle lighting.

\section{FEATURES}

- Available in white, outdoor white, 80-CRI, 85-CRI and 90-CRI white, royal blue, blue, green, amber, red \& red-orange

- Maximum drive current: up to $1 \mathrm{~A}$

- Low thermal resistance: as low as $9^{\circ} \mathrm{C} / \mathrm{W}$

- Maximum junction temperature: $150^{\circ} \mathrm{C}$

- Wide viewing angle: $115^{\circ}-130^{\circ}$

- Unlimited floor life at $\leq 30^{\circ} \mathrm{C} / 85 \% \mathrm{RH}$

- Reflow solderable - JEDEC JSTD-020C compatible

- Electrically neutral thermal path

- RoHS- and REACH-compliant

- UL-recognized component (E326295)

\section{TABLE OF CONTENTS}

Flux Characteristics - White ........ 2

Flux Characteristics - Color ......... 3

Characteristics ....................... 5

Relative Spectral Power

Distribution............................... 6

Relative Flux vs. Junction Tempera-

ture ...................................... 7

Electrical Characteristics............. 8

Thermal Design ....................... 9

Relative Flux vs. Current ............10

Relative Flux vs. Current ...........11

Typical Spatial Distribution..........11

Reflow Soldering Characteristics 12

Notes...................................13

Mechanical Dimensions...............14

Tape and Reel .......................15

Packaging...............................16 


\section{FLUX CHARACTERISTICS $\left(T_{3}=25^{\circ} \mathrm{C}\right)$ - WHITE}

The following table provides several base order codes for XLamp XP-E LEDs. It is important to note that the base order codes listed here are a subset of the total available order codes for the product family. For more order codes, as wel as a complete description of the order-code nomenclature, please consult the XLamp XP Family Binning and Labeling document.

\begin{tabular}{|c|c|c|c|c|c|}
\hline \multirow[t]{2}{*}{ Color } & \multicolumn{2}{|c|}{ CCT Range } & \multicolumn{2}{|c|}{$\begin{array}{l}\text { Base Order Codes } \\
\text { Min Luminous FFux } \\
\text { (Im) } \mathbf{3 5 0} \text { mA }\end{array}$} & \multirow[t]{2}{*}{ Order Code } \\
\hline & Min. & Max. & Group & Flux (Im) & \\
\hline \multirow{4}{*}{ Cool White } & \multirow{4}{*}{$5,000 \mathrm{~K}$} & \multirow{4}{*}{$10,000 \mathrm{~K}$} & Q4 & 100 & XPEWHT-L1-0000-00C01 \\
\hline & & & Q5 & 107 & XPEWHT-L1-0000-00D01 \\
\hline & & & R2 & 114 & XPEWHT-L1-0000-00E01 \\
\hline & & & R3 & 122 & XPEWHT-L1-0000-00F01 \\
\hline \multirow{4}{*}{$\begin{array}{l}\text { Outdoor } \\
\text { White }\end{array}$} & \multirow{4}{*}{$4,000 \mathrm{~K}$} & \multirow{4}{*}{$5,300 \mathrm{~K}$} & Q3 & 93.9 & XPEWHT-01-0000-00BC2 \\
\hline & & & Q4 & 100 & XPEWHT-01-0000-00CC2 \\
\hline & & & Q5 & 107 & XPEWHT-01-0000-00DC2 \\
\hline & & & R2 & 114 & XPEWHT-01-0000-00EC2 \\
\hline
\end{tabular}

\begin{tabular}{|c|c|c|c|c|c|}
\hline \multirow{3}{*}{$\begin{array}{l}\text { Neutral } \\
\text { White }\end{array}$} & \multirow{3}{*}{$3,700 \mathrm{~K}$} & \multirow{3}{*}{$5,000 \mathrm{~K}$} & Q3 & 93.9 & XPEWHT-L1-0000-00BE4 \\
\hline & & & Q4 & 100 & XPEWHT-L1-0000-00CE 4 \\
\hline & & & Q5 & 107 & XPEWHT-L1-0000-00DE 4 \\
\hline \multirow{3}{*}{$\begin{array}{l}\text { 80-CRI } \\
\text { White }\end{array}$} & \multirow{3}{*}{$2,600 \mathrm{~K}$} & \multirow{3}{*}{$4,300 \mathrm{~K}$} & P4 & 80.6 & XPEWHT-H1-0000-009E7 \\
\hline & & & Q2 & 87.4 & XPEWHT-H1-0000-00AE7 \\
\hline & & & Q3 & 93.9 & XPEWHT-H1-0000-00BE? \\
\hline \multirow{4}{*}{$\begin{array}{l}\text { Warm } \\
\text { White }\end{array}$} & \multirow{4}{*}{$2,600 \mathrm{~K}$} & \multirow{4}{*}{$3,700 \mathrm{~K}$} & P3 & 73.9 & XPEWHT-L1-0000-008E7 \\
\hline & & & P4 & 80.6 & XPEWHT-L1-0000-009E7 \\
\hline & & & Q2 & 87.4 & XPEWHT-L1-0000-00AE7 \\
\hline & & & Q3 & 93.9 & XPEWHT-L1-0000-00BE7 \\
\hline \multirow{3}{*}{$\begin{array}{l}85-C R I \\
\text { White }\end{array}$} & \multirow{3}{*}{$2,600 \mathrm{~K}$} & \multirow{3}{*}{$3,200 \mathrm{~K}$} & P2 & 67.2 & XPEWHT-P1-0000-007E7 \\
\hline & & & P3 & 73.9 & XPEWHT-P1-0000-008E7 \\
\hline & & & P4 & 80.6 & XPEWHT-P1-0000-009E7 \\
\hline \multirow{3}{*}{$\begin{array}{l}\text { 90-CRI } \\
\text { White }\end{array}$} & \multirow{3}{*}{$2,600 \mathrm{~K}$} & \multirow{3}{*}{$3,200 \mathrm{~K}$} & P2 & 67.2 & XPEWHT-U1-0000-007E7 \\
\hline & & & P3 & 73.9 & XPEWHT-U1-0000-008E7 \\
\hline & & & P4 & 80.6 & XPEWHT-U1-0000-009E7 \\
\hline
\end{tabular}

Notes:

- Cree maintains a tolerance of $\pm 7 \%$ on flux and power measurements and \pm 2 on CRI measurements.

- Typical CRI for Cool White \& Neutral White $(3,700 \mathrm{~K}-10,000 \mathrm{~K} \mathrm{CCT})$ is 75.

- Typical CRI for Outdoor White $(4,000 \mathrm{~K}-5,300 \mathrm{~K} \mathrm{CCT})$ is 70 .

- Typical CRI for Warm White $(2,600 \mathrm{~K}-3,700 \mathrm{~K} \mathrm{CCT})$ is 80 .

- Minimum CRI for 80 -CRI White is 80 .

- Minimum CRI for 85 -CRI White is 85 .

- Minimum CRI for 90-CRI White is 90. 


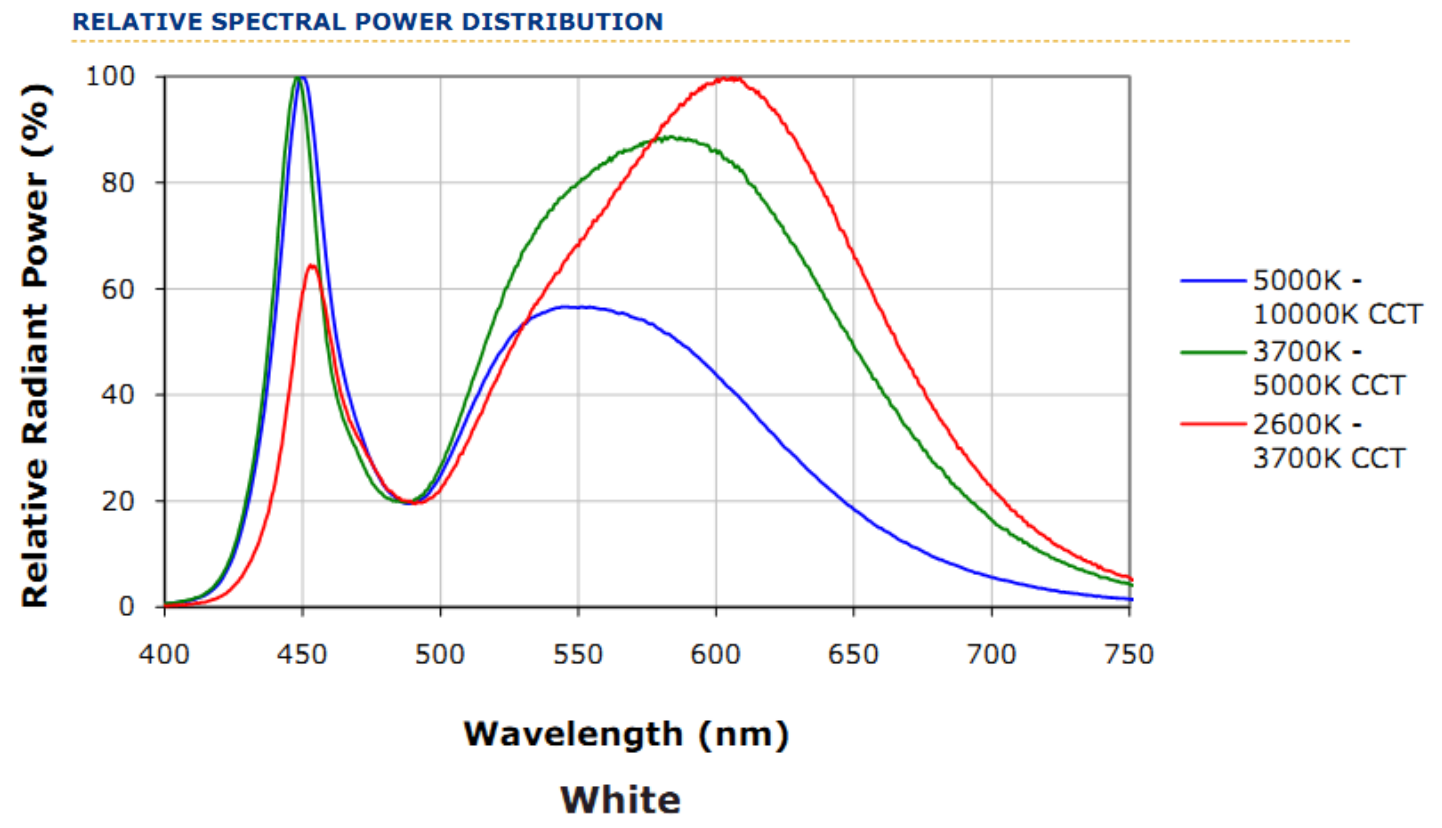

RELATIVE FLUX VS. JUNCTION TEMPERATURE ( $I_{F}=350$ MA $)$

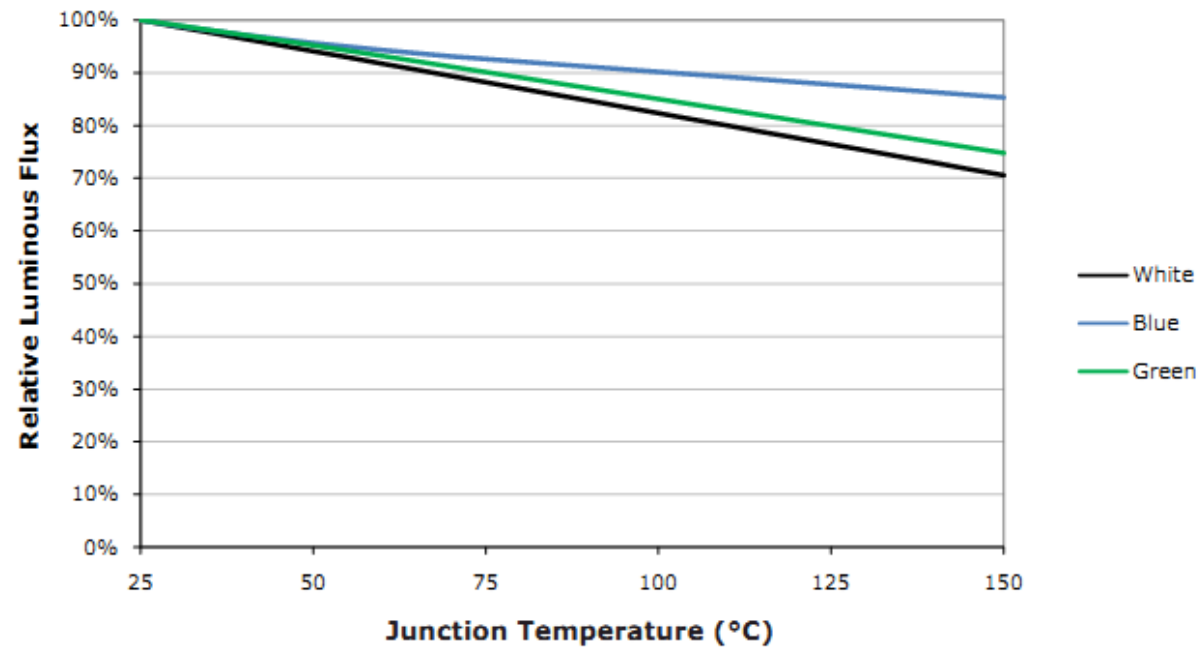

ELECTRICAL CHARACTERISTICS $\left(T_{3}=25^{\circ} \mathrm{C}\right)$

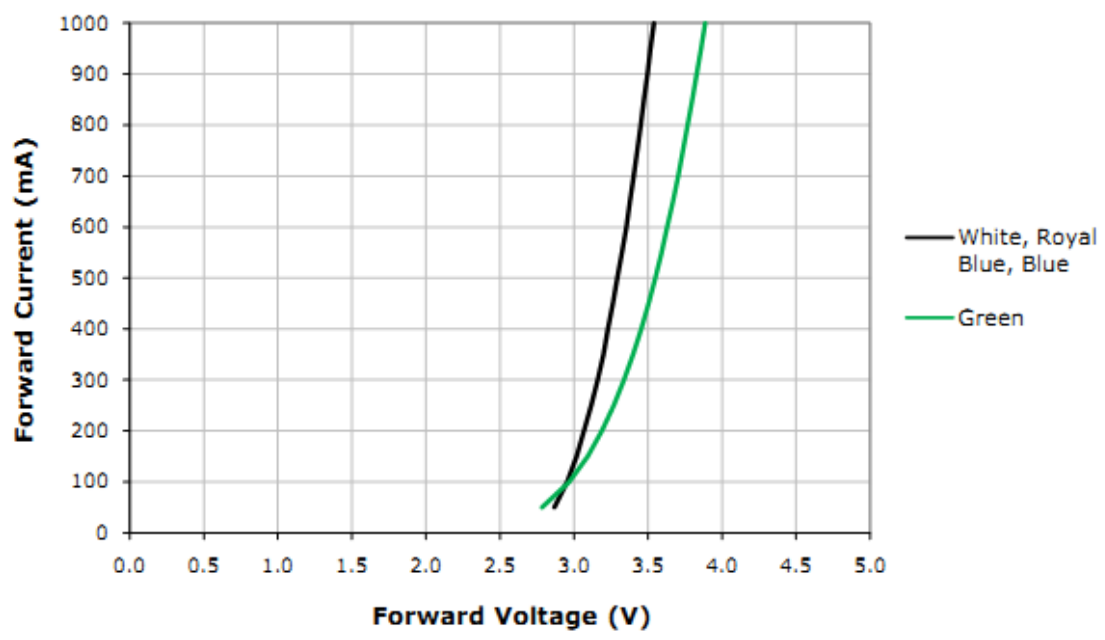




\section{THERMAL DESIGN}

The maximum forward current is determined by the thermal resistance between the LED junction and ambient. It is crucial for the end product to be designed in a manner that minimizes the thermal resistance from the solder point to ambient in order to optimize lamp life and optical characteristics.

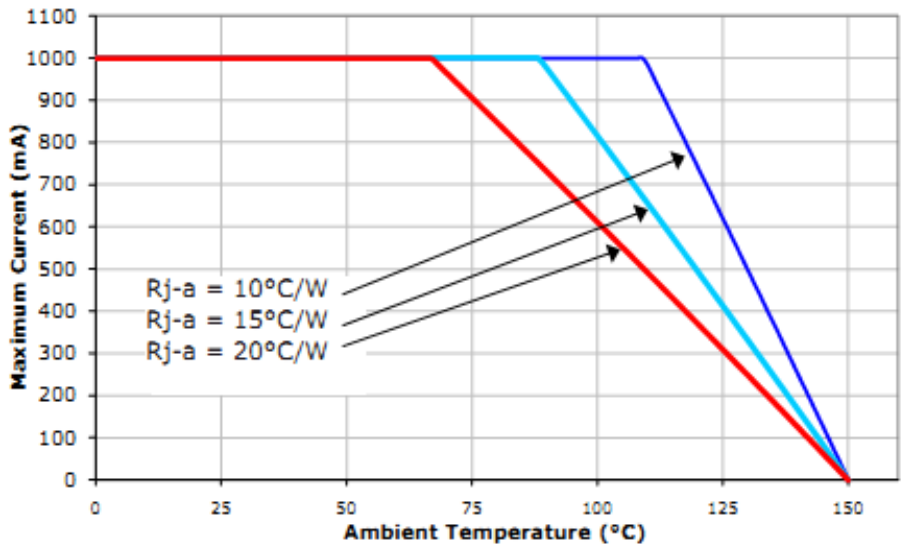

White, Blue and Royal Blue

RELATIVE FLUX VS. CURRENT $\left(T_{3}=25^{\circ} \mathrm{C}\right)$

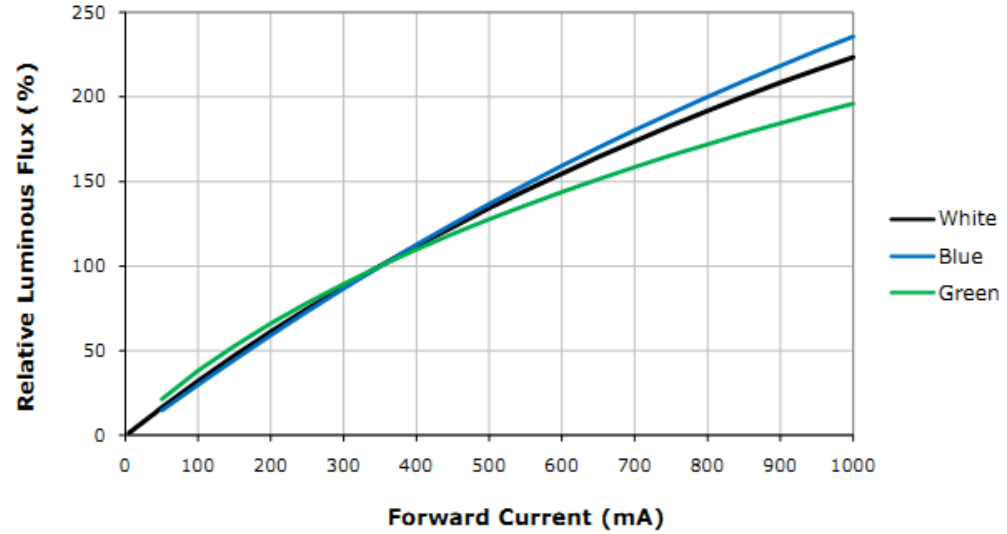

TYPICAL SPATIAL DISTRIBUTION

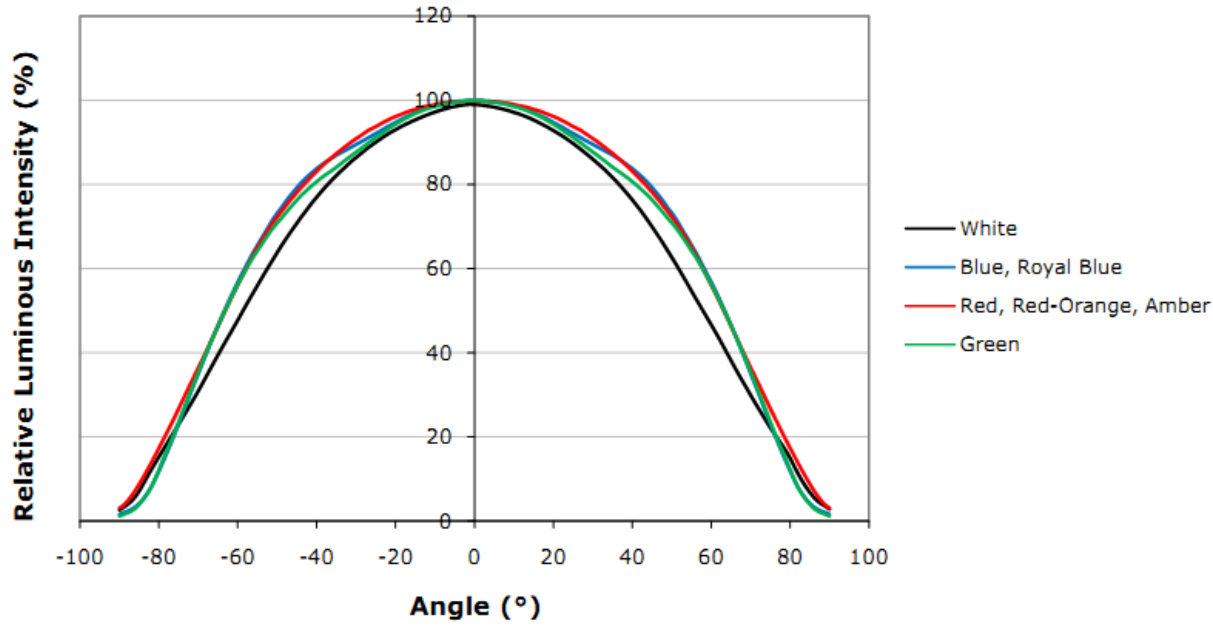




\section{NOTES}

Lumen Maintenance Projections

Based on internal long-term reliability testing, Cree projects royal blue, blue, green and white XLamp XP-E LEDs to maintain an average of $70 \%$ lumen maintenance after 50,000 hours, provided the LED junction temperature is maintained at or below $135^{\circ} \mathrm{C}$ and the LED is operated with a constant current of up to $700 \mathrm{~mA}$. Cree currently recommends a maximum drive current of $700 \mathrm{~mA}$ for XLamp XP-E white in designs seeking the ENERGY STAR* 35,000 hour lifetime rating ( $\geq 94.1 \%$ luminous flux@6000 hours) or 25,000 hour lifetime rating ( $\geq 91.8 \%$ luminous flux@6000hours).

Please read the XLamp Long-Term Lumen Maintenance application note for more details on Cree's lumen maintenance testing and forecasting. Please read the XLamp Thermal Management application note for details on how thermal design, ambient temperature, and drive current affect the LED junction temperature.

* These lifetime ratings are based on the current ENERGY STAR Solid State Lighting Luminaires V1.1 (December 12, 2008) and ENERGY STAR Integral LED Lamps V1.0 (December 3, 2009) lumen maintenance criteria.

\section{Moisture Sensitivity}

In testing, Cree has found XLamp XP-C \& XP-E LEDs to have unlimited floor life in conditions $\leq 30^{\circ} \mathrm{C} / 85 \%$ relative hu-midity (RH). Moisture testing included a 168 hour soak at $85^{\circ} \mathrm{C} / 85 \% \mathrm{RH}$ followed by 3 reflow cycles, with visual and electrical inspections at each stage.

\section{RoHS Compliance}

The levels of environmentally sensitive, persistent biologically toxic (PBT), persistent organic pollutants (POP), or other-wise restricted materials in this product are below the maximum concentration values (also referred to as the threshold limits) permitted for such substances, or are used in an exempted application, in accordance with EU Directive 2002/95/EC on the restriction of the use of certain hazardous substances in electrical and electronic equipment (RoHS), as amended through April 21, 2006.

Vision Advisory Claim WARNING: Do not look at exposed lamp in operation. Eye injury can result. See LED Eye Safety at http://www.cree.com/products/pdf/XLamp_EyeSafety.pdf.

Intellectual Property

For remote phosphor applications, a separate license to certain Cree patents is required. 

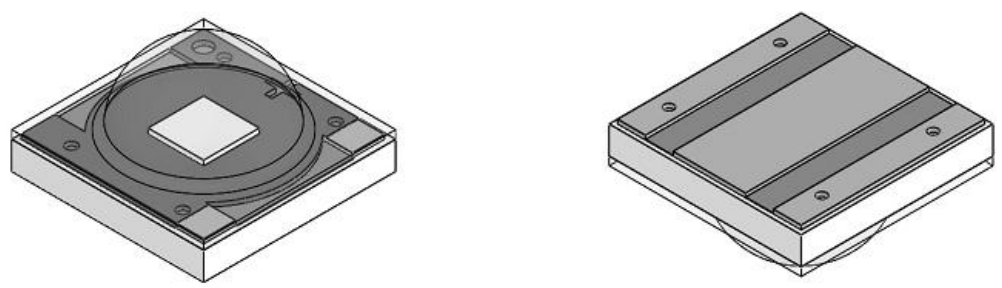

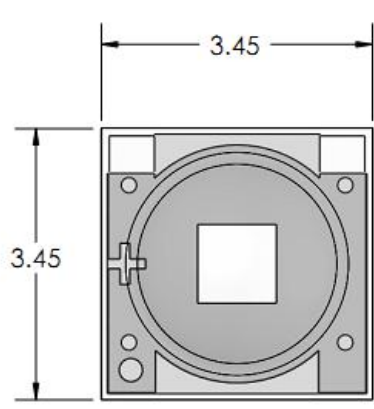

Top View

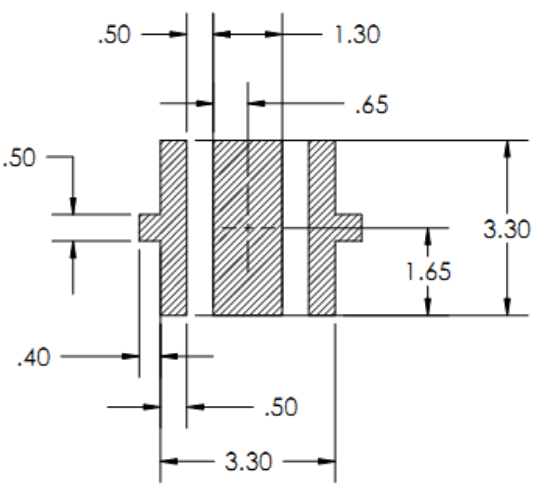

RECOMMENDED PCB SOLDER PAD

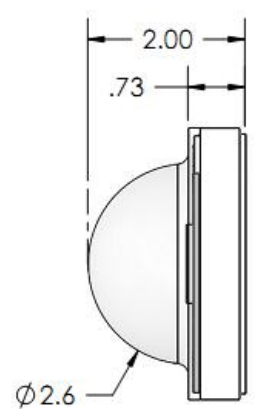

Side View
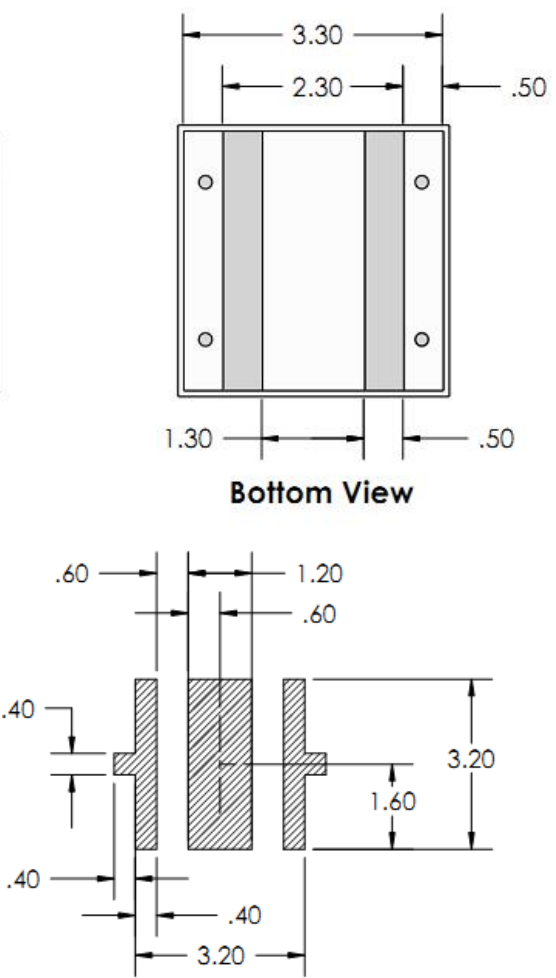

RECOMMENDED STENCIL PATTERN (HATCHED AREA IS OPENING)

Illuminance meter (body serial number 42921027, measuring head serial number 62931034:

Minolta T-10 (Konica Minolta website)

\section{Uniform luminance source (ULS):}

(Labsphere website)

ULS is a special type of integrating sphere that has two lamps. The primary lamp is located outside of the sphere. Power supplies are used to drive the lamps. As specified by the manufacturer lamps should be driven by 20 volts and 5.7 amps (114 watts). The color temperature is $2856 \mathrm{~K}$. The change in power will alter the color temperature of the light source. In order to avoid these effects one has to change the 
aperture, which will allow more or less light to enter the sphere and to result in different luminous levels. Aperture size dictates the amount of light entering the sphere. Baffle inside the sphere acts like a diffuser which results in a uniform luminance level. The purpose of the second lamp is to increase luminance level if necessary, which was not in this case. The source lamps in the uniform light source sphere are incandescent lamps. They emit a broadband spectrum to represent all wavelengths for calibration. Picoammeter receives a reading from a photometric head and shows luminance value of the sphere.

\section{Luminance meter (serial number 79923018):}

(Konica Minolta website)

LS-110

Acceptance area: $1 / 3^{\circ}$

Field of view: $9^{\circ}$

Focusing distance: $1014 \mathrm{~mm}$ to infinity (447 minimum with close-up lens)

Measuring range: Fast: 0.01 to $999900 \mathrm{~cd} / \mathrm{m}^{2}$

Accuracy: $0.01-9.99 \mathrm{~cd} / \mathrm{m}^{2} \pm 2 \% \pm 2$ digits of measured value

$10.00 \mathrm{~cd} / \mathrm{m}^{2}$ or more $\pm 2 \% \pm 1$ digit of measured value

(Measuring conditions: Standard Illuminant A, ambient temperature: 20 to $30^{\circ} \mathrm{C}$ )

Optical system $85 \mathrm{~mm} \mathrm{f/2.8} \mathrm{lens}$

Luminance meter was calibrated for 12/8/11-12/8-12 by Konica Minolta

Table B. Close-up lense

\begin{tabular}{|c|l|l|l|l|}
\hline $\mathbf{m m}$ & $\begin{array}{l}\text { (a)Measuring } \\
\text { diameter at } \\
\text { minimum } \\
\text { measuring } \\
\text { distance }\end{array}$ & $\begin{array}{l}\text { (b)Measuring } \\
\text { diameter at } \\
\text { maximum } \\
\text { measuring } \\
\text { distance } \\
\text { lens }\end{array}$ & $\begin{array}{l}\text { (c)Minimum } \\
\text { measuring } \\
\text { distance }\end{array}$ & $\begin{array}{l}\text { (d)Maximum } \\
\text { measuring } \\
\text { distance }\end{array}$ \\
\hline None & $\Phi 4.8$ & - & 1014 & $\infty$ \\
\hline$\# 135$ & $\Phi 1.8$ & $\Phi 2.9$ & 447 & 615 \\
\hline
\end{tabular}

Color-correction factor should be set to 1.05 to adjust the response of the meter to compensate for the close-up lens. 


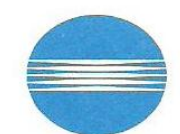

KONICA MINOLTA

\section{CERTIFICATE OF CALIBRATION}

Issued By

Konica Minolta Sensing Americas, Incorporated

101 Williams Drive, Ramsey, New Jersey 07446

Voice (201) 785-2462 Facsimile (201) 785-2490

Submitted By: University of Nebraska.

Certificate Number: KMSA-001-00-008393.

Instrument: Luminance Meter, LS-110. Serial Number: 79923018.

This certificate is provided for the Luminance Meter listed above to confirm that the performance and accuracy of said instrument is within KONICA MINOLTA'S WORKING STANDARDS which are traceable to the National Institute of Standards and Technology (NIST), Test Number 685/280718-11.

\section{**STATEMENT OF CALIBRATION CONDITIONS **}

In compliance with your request concerning calibration of above Luminance Meter, Konica Minolta provides the following information:

a) The instrument is calibrated to a KONICA MINOLTA STANDARD tungsten filament lamp for color and luminous intensity. The lamp is illuminant standard "A" calibrated to our primary standard lamp (\#5E-103K/153) which has been certified by N.I.S.T. for Color Temperature and Luminous Intensity.

b) The voltage meter and the power supply used on the optical bench were last calibrated in February 2011.

c) Our KONICA MINOLTA STANDARD tungsten filament lamps are rotated and recalibrated after accumulating 24 hours of use.

d) Our calibration facility is environmentally controlled to maintain a temperature between $69-74 \mathrm{~F}$ with a humidity of approximately $45 \%-65 \%$.

e) Uncertainty coverage factor ( $\mathrm{k}=2)$ Uncertainty: $(1.3 \%)$ Range: $10 \mathrm{~cd}--3000 \mathrm{~cd}$ For Luminous intensity.

f) Our recommended cycle of calibration is a twelve month cycle.

CALIBRATION STANDARD $\quad$ PREVIOUS READING $\quad$ NEW READING

$\underline{Y=145.1 \mathrm{~cd} / \mathrm{m} 2(+-2 \%)} \quad \underline{\mathrm{Y}=141.8 \mathrm{~cd} / \mathrm{m} 2(\mathrm{In} \mathrm{Tol})} \quad \underline{\mathrm{Y}=145.1 \mathrm{~cd} / \mathrm{m} 2}$

Date Received:_December 2, 2011. Date Calibrated: December 8, 2011.

Date Calibration Due: December 8, 2012.

Certified By:_S. Politza. Title: Service Repair Engineer.

**THIS CERTIFICATE SHALL NOT BE REPRODUCED EXCEPT IN FULL, WITHOUT THE WRITTEN APPROVAL OF THIS LABORATORY**

KONICA MINOLTA SENSING AMERICAS, INC. 101 Williams Drive, Ramsey, NJ 07446-1293 Tel: 201-236-4300 - Fax: 201-785-2480 - Toll Free: 888-473-2656

Figure B. Calibration certificate for LS110 luminance meter 


\section{Appendix C: LED output measurements depending on line voltage over the day-time period}

LS110 measured the luminance values of a white reflectance standard (99\%) set in the room with only single LED chip illuminating it. FLUKE 87 III True RMS Multimeter is measuring line voltage. LED was turned on at 10:46am.

Table C. Luminance of reflectance standard (99 \%) illuminated by a single LED chip depending on line voltage over a day-time period

\begin{tabular}{|c|c|c|}
\hline Time of the day & $\begin{array}{c}\mathrm{L}, \mathrm{cd} / \mathrm{m}^{2} \text { (reflectance } \\
\text { standard } 99 \% \\
\text { illuminated by a single } \\
\text { LED chip) }\end{array}$ & Voltage, $\mathrm{V}$ \\
\hline $10: 46 \mathrm{am}$ & 15.96 & 118.4 \\
\hline $10: 50 \mathrm{am}$ & 15.96 & 118.4 \\
\hline $10: 58 \mathrm{am}$ & 16.01 & 118.1 \\
\hline 11:01 am & 15.76 & 118.1 \\
\hline 11:06 am & 15.73 & 118.2 \\
\hline $11: 12 \mathrm{am}$ & 15.70 & 118.1 \\
\hline 11:19 am & 15.61 & 118.1 \\
\hline $11: 23 \mathrm{am}$ & 15.54 & 118.2 \\
\hline $1: 09 \mathrm{pm}$ & 15.15 & 118.1 \\
\hline $1: 27 \mathrm{pm}$ & 15.09 & 118.0 \\
\hline $1: 52 \mathrm{pm}$ & 15.09 & 118.2 \\
\hline $2: 22 \mathrm{pm}$ & 15.11 & 118.2 \\
\hline $2: 47 \mathrm{pm}$ & 15.12 & 118.2 \\
\hline $3: 12 \mathrm{pm}$ & 15.07 & 118.5 \\
\hline $3: 37 \mathrm{pm}$ & 15.1 & 118.5 \\
\hline $4: 01 \mathrm{pm}$ & 15.1 & 118.5 \\
\hline $4: 28 \mathrm{pm}$ & 15.12 & 118.5 \\
\hline $4: 59 \mathrm{pm}$ & 15.9 & 118.3 \\
\hline $5: 27 \mathrm{pm}$ & 15.1 & 118.4 \\
\hline $5: 49 \mathrm{pm}$ & 15.13 & 118.4 \\
\hline $6 \mathrm{pm}$ & 15.1 & 118.4 \\
\hline
\end{tabular}




\section{Appendix D: Excerpt from HDRI Mailing Lists (www.radiance- online.org)}

For more information please refer to the HDRI mailing lists website.

\section{October 2011}

Response curve from Photosphere

Tyukhova, Yulia ytyukhova at unomaha.edu Tue Oct 25 15:00:13 PDT 2011

Does anybody know how to get the information on the response curve from Photosphere similar to the one that WebHDR would give you?

Thank you,

Yulia

Gregory J. Ward gregoryjward at gmail.com

Tue Oct 25 15:46:50 PDT 2011

Hi Yulia,

The camera response functions are stored in the text file \$HOME/Library/Preferences/Photosphere

Towards the end, you will see a list of camera responses that have been stored by make, model, and version. Each line looks something like this:

“NIKON CORPORATION”|'NIKON D200”|"Ver.2.01”|\{-4.765716e-04,1.355876e$01,-1.269868 \mathrm{e}-01,9.918758 \mathrm{e}-01\} \mid\{-6.390453 \mathrm{e}-04,1.371893 \mathrm{e}-01,-1.224765 \mathrm{e}-$ $01,9.859263 \mathrm{e}-01\} \mid\{-9.074065 \mathrm{e}-04,1.351222 \mathrm{e}-01,-1.165654 \mathrm{e}-01,9.823506 \mathrm{e}-01\}$

The coefficients enclosed in curly braces are the polynomial camera responses, one per color in red, green, and blue order. The coefficient order is from low to high power, so the above line translates to:

$\operatorname{red}(\mathrm{r})=-4.765716 \mathrm{e}-04+1.355876 \mathrm{e}-01 * \mathrm{r}+-1.269868 \mathrm{e}-01 * \mathrm{r}^{\wedge} 2+9.918758 \mathrm{e}-01 * \mathrm{r}^{\wedge} 3$ $\operatorname{green}(\mathrm{g})=-6.390453 \mathrm{e}-04+1.371893 \mathrm{e}-01 * \mathrm{~g}+-1.224765 \mathrm{e}-01 * \mathrm{~g}^{\wedge} 2+9.859263 \mathrm{e}-$ $01 * \mathrm{~g}^{\wedge} 3$

blue $(b)=-9.074065 e-04+1.351222 \mathrm{e}-01 * b+-1.165654 \mathrm{e}-01 * \mathrm{~b}^{\wedge} 2+9.823506 \mathrm{e}-$ $01 * b^{\wedge} 3$

The input $(\mathrm{r}, \mathrm{g}, \mathrm{b})$ values are camera sensor values normalized in a $0-1$ range. The output values are related to world luminance units, sometimes with a calibration scaling factor built in.

I hope this is helpful.

-Greg

\section{February 2012}


Tyukhova, Yulia ytyukhova at unomaha.edu

Sun Feb 19 21:34:25 PST 2012

Hello everybody!

I'll provide the summary of my research and have questions within the summary. I would appreciate any of your help!

My research investigates if HDRI technique can precisely capture luminances of small bright light sources (e.g. LED garage fixtures) with narrow light distributions.

I was able to figure out luminance values for a single LED, which can be compared to the ones from HDR images. But I have a couple of questions/concerns on HDRI technique and Photosphere.

At first, I've used "regular" scene to retrieve response curve of the camera (large smooth gradients with very dark and bright areas, and had reflectance standards for the absolute calibration).

Camera: EOS T1i Rebel with $28-105 \mathrm{~mm}$ lens, at $28 \mathrm{~mm}$ Calibrated at the grey reflectance standard $186.45 \mathrm{~cd} / \mathrm{m}^{2}$

$\mathrm{CF}=0.957$

I've got the following RC for RGB:

$\operatorname{red}(\mathrm{r})=-6.434199 \mathrm{e}-03+4.518039 \mathrm{e}-01 * \mathrm{r}+1.291426 \mathrm{e}+00 * \mathrm{r}^{\wedge} 2+1.802896 \mathrm{e}+00$

${ }^{*} \mathrm{r}^{\wedge} 3$;

$\operatorname{green}(\mathrm{g})=-5.804720 \mathrm{e}-03+4.175837 \mathrm{e}-01 * \mathrm{~g}+1.176582 \mathrm{e}+00 * \mathrm{~g}^{\wedge} 2+1.721643 \mathrm{e}+00$ $*_{\mathrm{g}} \wedge 3$;

blue $(b)=-4.376831 e-03+3.784418 \mathrm{e}-01 * b+1.075695 e+00 * b^{\wedge} 2+1.658471 e+00$ $* b^{\wedge} 3$

If I look at the histogram of the scene, maximum luminance within the scene is $60,291 \mathrm{~cd} / \mathrm{m} 2$.

Then I use this RC to analyze HDRI of a captured LED. The value is $230,000 \mathrm{~cd} / \mathrm{m}^{2}$ for a single LED, which is low (it's has to be around $7 * 106 \mathrm{~cd} / \mathrm{m}^{2}$ ). So, it underestimates the luminance.

It seems like calibration point is critical here. I've decided to try to capture a different scene for deriving $\mathrm{RC}$ with a wider range. It would make sense that camera has to see higher luminance values in order to accurately measure them later. The dynamic range has to cover measured values.

1. How does Photosphere deals/approximates/calculates the upper end of the curve? I assume it gives more weight to mid tone values? But what happens with high luminance values?

So, the new brighter scene was picked with the direct sun! But in order to avoid the damage of the camera's sensor, measurements were taken before the sunset. 
In the new brighter captured scene without the calibration all values for reflectance standards were overestimated, while the value for the sun underestimated. Then I decided to calibrate my scene at the sun!

But when I apply absolute calibration, it simply multiplies CF to all values.

I assumed when CF is applied, it does not equally change all values, but does it proportionally to $\mathrm{RC}$ (since it is not linear). Why does it do it equally for the whole range?

Lsun=80*106 cd/m2. And of course CF is very big 391 .

New RC:

$\operatorname{red}(\mathrm{r})=3.219064 \mathrm{e}+00+-2.655078 \mathrm{e}+01 * \mathrm{r}+9.351069 \mathrm{e}+02 * \mathrm{r}^{\wedge} 2+-2.115052 \mathrm{e}+03$

$* \mathrm{r}^{\wedge} 3+1.594538 \mathrm{e}+03 * \mathrm{r}^{\wedge} 4$

green $(\mathrm{g})=2.094164 \mathrm{e}+00+-1.468109 \mathrm{e}+00 * \mathrm{~g}+7.306838 \mathrm{e}+02 * \mathrm{~g} \wedge 2+-1.720743 \mathrm{e}+03$

$* \mathrm{~g}^{\wedge} 3+1.380693 \mathrm{e}+03 * \mathrm{~g}^{\wedge} 4$

blue $(b)=1.049078 \mathrm{e}+00+1.591820 \mathrm{e}+01 * \mathrm{~b}+5.848958 \mathrm{e}+02^{*} \mathrm{~b}^{\wedge} 2+-1.461635 \mathrm{e}+03$

$* b^{\wedge} 3+1.251033 \mathrm{e}+03 * \mathrm{~b}^{\wedge} 4$

But then something interesting happened. When I analyze LED, it gives a value of $79 * 106 \mathrm{~cd} / \mathrm{m}^{2}$. So, it jumps to this upper limit calibrated with the sun previously.

(I had similar results for EOS 7D with the lens 16-35mm, at 16mm)

Does photosphere compress the response curve, so at the upper end all values above certain threshold will have the same number?

Any additional suggestions on properly obtaining and calibrating HDRI for this purpose?

Gregory J. Ward gregoryjward at gmail.com

Mon Feb 20 10:10:40 PST 2012

Hello Yulia,

Seems your question has spawned quite a bit of interesting discussion...

My main recommendation is to use camera RAW images for critical photometry, especially when there are saturated colors involved. It is impossible to correct the color of JPEG images and undo what the camera maker has done, so you need to start from the sensor data.

Photosphere does not accept camera RAW as input, but I have written a Perl script that uses dcraw with the command-line HDR image builder hdrgen to overcome this limitation. It also requires the use of another third-party program, exiftool, which I have packaged together for you at: 
Unfortunately, I do not have a good set of documentation to go with it. Typing "raw2hdr" by itself shows the basic syntax:

Usage: raw2hdr [hdrgen opts][-h][-w][-C calib][-c cspace] -o output.hdr input1.raw ..

If your images are taken on a tripod (aligned exposures), you can use the default settings:

raw2hdr -o output.hdr expos1.cr2 expos2.cr2 expos3.cr2 ...

The hdrgen settings can be found in the included HTML man page, and so can the $-\mathrm{h}$ and $-\mathrm{w}$ option meanings in the included dcraw man page. The $-\mathrm{C}$ option is to provide a linear factor to correct the overall exposure based on previous calibrations. The $-\mathrm{c}$ option is to specify an output color space. The default is "sRGB" which is actually linear CCIR-709 primaries. The only other output color space I would recommend is AdobeRGB. There is a CIE XYZ space supported by dcraw, but I have found it to be somewhat unreliable, and I do not know where the fault lies in this.

Regarding Axel's mention of camera flare, this is less of an issue for sources that are brighter than the rest of the scene. It mostly affects darker, surrounding regions. The -f option will attempt to estimate the camera/lens PSF and remove it, but it cannot be relied upon to remove this source of error completely. Your problem with the accuracy of the LED sources is due no doubt (as others have said) to limitations in your short exposures combined with the color issues inherent to JPEG processing.

Other responses inline....

>1. How does Photosphere deals/approximates/calculates the upper end of the curve? I assume it gives more weight to mid tone values? But what happens with high luminance values?

Photosphere (and hdrgen) use all the brightest pixels from the shortest exposure and all the darkest pixels from the longest exposure. Middle exposures have their brightest and darkest pixels downgraded.

I do not think your shortest exposure properly captured the LED, and maybe didn't capture the sun, either.

> 3. Does photosphere compress the response curve, so at the upper end all values above certain threshold will have the same number?

Photosphere does not compress the curve.

>4. Any additional suggestions on properly obtaining and calibrating HDRI for this purpose?

I would only reiterate others' suggestion to use a neutral density filter, and using raw2hdr rather than Photosphere.

Best,

-Greg 
Tyukhova, Yulia ytyukhova at unomaha.edu

Mon Feb 20 12:48:37 PST 2012

Everybody,

Thank you for fast responses/tools and suggestions!

Greg,

Thank you for your suggestions and files!

I am new to Radiance, and I assume that this is what I need to have installed on my computer in order to use suggested Perl scripts.

If you can provide me with the link/info how to run it, that would be really helpful!

Let me restate the question about the compression of the curve in Photosphere.

Do manufactures compress the response curve or maybe it is limited by camera/optics/sensor saturation itself on the upper end?

And I'm still curious, how CF is applied in Photosphere?

I've been using ND filter $\mathrm{t}=0.0094$ on the luminance meter, because otherwise it is impossible to measure such high luminances. I assume, you suggest to use it on the camera as well.

I'm looking forward to analyze my images with the suggested hdrgen. Luckily, I've been taken them in both formats jpeg and raw. Greg, will you recommend to have regular calibration scene calibrated at the grey card instead of using brighter scene?

Thank you, Yulia

Gregory J. Ward gregoryjward at gmail.com Mon Feb 20 13:14:15 PST 2012

Responses inline...

$>$ I am new to Radiance, and I assume that this is what I need to have installed on my computer in order to use suggested Perl scripts.

Actually, you do not need to have Radiance installed. You just need to move the executables (non-HTML files) from the unpacked directory to /usr/bin or /usr/local/bin or some other directory in your shell's PATH variable. These are command-line tools that must be run from the Terminal application under /Applications/Utilities. I.e., start Terminal and copy the files from your Downloads folder with:

cd Downloads

tar xzf raw2hdr.tgz

cd raw2hdr 


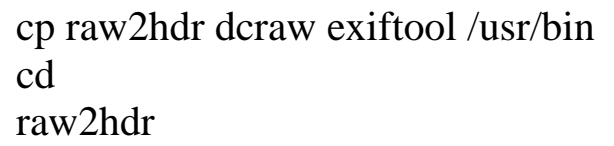

This should give you the usage message I wrote you earlier if it all goes well. Some basic commands and pointers for Unix are available many places online. Googling "basic unix tutorial" gave this page at the head of the list:

\section{http://www.ee.surrey.ac.uk/Teaching/Unix/}

$>$ Let me restate the question about the compression of the curve in Photosphere.

$>1$. Do manufactures compress the response curve or maybe it is limited by camera/optics/sensor saturation itself on the upper end?

Some camera makers do compress the top end of the response curve, and do funny things at the bottom as well. Photosphere attempts to discover the tone curve and correct for these manipulations, but it isn't perfect and if the camera is changing the tone curve dynamically, it's pretty hopeless. There are settings you can use on a DSLR to disable such manipulations, but using RAW files bypasses the problems entirely because the data is linear.

\section{> 2. And I'm still curious, how CF is applied in Photosphere?}

A calibration factor is applied equally to all coefficients in the polynomial, which is exactly the same as applying a linear scale factor after the HDR merge operation.

$>$ I've been using ND filter $t=0.0094$ on the luminance meter, because otherwise it is impossible to measure such high luminances. I assume, you suggest to use it on the camera as well.

Whatever gives you a short exposure that is past the integration time of your source $\left(1 / 60^{\text {th }}\right.$ second is acceptable) and not saturated is OK. Specifically, all values in the short exposure's histogram should be be below 245 .

> I'm looking forward to analyze my images with the suggested hdrgen. Luckily, I've been taken them in both formats jpeg and raw.

> Greg, will you recommend to have regular calibration scene calibrated at the grey card instead of using brighter scene?

The best scene for calibration is a white card in a scene with no bright sources directed at the camera. The calibration should hold in other scenes where lens flare is not problematic.

Certainly,

-Greg

Tyukhova, Yulia ytyukhova at unomaha.edu

Tue Feb 21 13:21:05 PST 2012

Greg,

Following up your suggestions... 
I was able to run your scripts, but now I have more questions on what's behind them.

I assume after combining raw images into one, I can analyze obtained hdr image in Photosphere.

$>-r *$ cam.rsp*

Use the given file for the camera's response curves. If this file exists, it must contain the coefficients of three polynomials, one for each color primary. If the file does not exist, hdrgen will use its principal algorithm to derive these coefficients and write them out to this file for later use.

So, if I will combine my images for the calibration scene, hdrgen will derive the coefficients to the file. Is there a way to see those coefficients and to know what the response curve looks like?

$>$ The - C option is to provide a linear factor to correct the overall exposure based on previous calibrations.

How do I make the absolute calibration? Usually it is a luminance value of a reflectance standard measured with luminance meter applied in Photosphere. But how do I do it with the given script?

> The -c option is to specify an output color space. The default is «sRGB» which is actually linear CCIR-709 primaries. The only other output color space I would recommend is AdobeRGB. There is a CIE XYZ space supported by dcraw, but I have found it to be somewhat unreliable, and I do not know where the fault lies in this.

In order to have luminance values Photosphere has an algorithm that does color calculations from sRGB to CIE XYZ (standard illuminant D65), where Y is the luminance value. Here I can specify an output color space, let's say sRGB, but how would I get luminance values?

$>-S$ *stonits*

5.What is this option about?

Gregory J. Ward gregoryjward at gmail.com

Tue Feb 21 13:55:58 PST 2012

Hi Yulia,

Not all of the options of hdrgen are relevant for raw2hdr. See inline....

> 1. I assume after combining raw images into one, I can analyze obtained hdr image in Photosphere.

Yes, of course.

$>>>-r$ cam.rsp 
$>$ Use the given file for the camera's response curves. If this file exists, it must contain the coefficients of three polynomials, one for each color primary. If the file does not exist, hdrgen will use its principal algorithm to derive these coefficients and write them out to this file for later use.

$>$

> 2. So, if I will combine my images for the calibration scene, hdrgen will derive the coefficients to the file. Is there a way to see those coefficients and to know what the response curve looks like?

The raw2hdr script doesn't need to derive a response curve, since the sensor data is linear. Instead, it creates an output from dcraw that follows a 2.0 gamma and creates an artificial response curve of $x^{\wedge} 2$ to decode it. This reduces quantization errors from the 8-bit intermediate images.

$>>>$ The - C option is to provide a linear factor to correct the overall exposure based on previous calibrations.

$>$

> 3. How do I make the absolute calibration? Usually it is a luminance value of a reflectance standard measured with luminance meter applied in Photosphere. But how do I do it with the given script?

Look at your raw2hdr result in Photosphere and select the measured area. Divide your measurement by the value Photosphere gives you. This is the calibration factor to use with the $-\mathrm{C}$ option for conversions for this camera and lens.

> >> The -c option is to specify an output color space. The default is "sRGB" which is actually linear CCIR-709 primaries. The only other output color space I would recommend is AdobeRGB. There is a CIE XYZ space supported by dcraw, but I have found it to be somewhat unreliable, and I do not know where the fault lies in this. $>$

$>4$. In order to have luminance values Photosphere has an algorithm that does color calculations from sRGB to CIE XYZ (standard illuminant D65), where $Y$ is the luminance value. Here I can specify an output color space, let's say sRGB, but how would I get luminance values?

As I mentioned, the sRGB and AdobeRGB spaces will both work, and Photosphere will adjust its $\mathrm{Y}$ value calculations accordingly. The color space is recorded in the HDR output.

$>>-$ s stonits

$>$ 5. What is this option about?

You do not need it - this option is for when the camera doesn't record the necessary aperture, asa and shutter speed settings in the image file.

Best,

-Greg

Tyukhova, Yulia ytyukhova at unomaha.edu

Wed Feb 22 12:59:45 PST 2012

Hello Greg!

I was able to compile my images of LED with ND filter with raw2hdr, but I 
need to clarify a couple of things.

As the intermediate step of combining the images I have the following:

Writing data to standard output ...

Can’t locate Image/ExifTool.pm in @INC (@INC contains: /usr/bin/lib

/Library/Perl/5.12/164arwin-thread-multi-2level /Library/Perl/5.12

/Network/Library/Perl/5.12/164arwin-thread-multi-2level

/Network/Library/Perl/5.12/Library/Perl/Updates/5.12.3

/System/Library/Perl/5.12/164arwin-thread-multi-2level

/System/Library/Perl/5.12

/System/Library/Perl/Extras/5.12/164arwin-thread-multi-2level

/System/Library/Perl/Extras/5.12 .) at/usr/bin/exiftool line 30.

BEGIN failed-compilation aborted at/usr/bin/exiftool line 30.

Loading Canon EOS 7D image from IMG_0279.CR2 ...

What does it mean? Is there a problem with data input/output? I just want to make sure that the data is processed properly.

At first it didn't make sense why do I need hdrgen, since it uses tiff or jpeg as an input while I'm combining hdr from raw. But then I've noticed that raw $2 \mathrm{hdr}$ generates temporary tiff photos and then uses them in hdrgen function. But if I want to include some additional settings for hdrgen (like flare removal) besides the default ones I have to following error: raw2hdr hdrgen $-\mathrm{f}-\mathrm{o}$ output6.hdr IMG_02??.CR2 Missing -o output file specification

How do I write my settings?

Thank you!

Yulia

Gregory J. Ward gregoryjward at gmail.com

Wed Feb 22 13:50:59 PST 2012

Yulia,

The error with Exiftool is my fault, I'm afraid. I naively thought that the program was self-contained, when it is not. You need to download and install it on your machine via the following URL:

\section{http://www.sno.phy.queensu.ca/ phil/exiftool/install.html}

As for your second question, you can use other hdrgen options, but you do not need to write "hdrgen" on the command line as you have done. The raw2hdr script knows how to sort out the various options.

Best,

-Greg

HDRI LED capture interesting artifacts

Tyukhova, Yulia ytyukhova at unomaha.edu

Thu Feb 23 09:10:13 PST 2012 
Hello!

I've got some interesting artifacts on my images of a single LED, and I'm curious how to interpret and overcome them.

Please see two images at:

http://www.mediafire.com/?6w6tfmpoqtpipap,c0e69acf1x51ndu

The settings for taking both are the following:

*1 ND filter output7.jpg*

Range of shutter speed $1 / 8000$ to $1 / 15^{\prime \prime}$ ' with aperture f16

Range $1 / 30$ ' to 15 ' with aperture $\mathrm{f} 4$

Luminance of reflectance standards:

$99 \% \quad 197.2 \mathrm{~cd} / \mathrm{m}^{\wedge} 2$

$40 \% \quad 99.3 \mathrm{~cd} / \mathrm{m}^{\wedge} 2$

ND filter $\mathrm{t}=0.0094$

ND filters output14.jpg*

Range $1 / 8000$ to $1 / 15^{\prime}$ ' with aperture f16

Range $1 / 30$ ' to 15 ' with aperture $\mathrm{f} 4$

Ambient light level (illuminance meter) E=667 lx

Luminance of reflectance standards:

$99 \% 215 \mathrm{~cd} / \mathrm{m}^{\wedge} 2$

$40 \% \quad 106.3 \mathrm{~cd} / \mathrm{m}^{\wedge} 2$

ND filter $\mathrm{t}=0.0094$

$2^{\text {nd }} \mathrm{ND}$ filter $\mathrm{t} 2=0.4375$

Thank you,

Yulia

Gregory J. Ward gregoryjward at gmail.com

Thu Feb 23 09:42:59 PST 2012

Hi Yulia,

There is an occasional issue with dcraw where it doesn't quite handle the highlights correctly, leaving this pink area near the brightest part of the exposure. The only fix I've found is to set the $-\mathrm{b}$ option to a larger value. You should try this with raw2hdr. Try "raw2hdr -b 1.3 ..." adding your other options as usual. If that doesn't get rid of the pink haloes, keep increasing the value until it does.

If this doesn't work, then maybe you need more exposures. I'm not really sure unless I can play with your original RAW files what else it could be.

Best,

-Greg

Axel Jacobs jacobs.axel at gmail.com

Mon Feb 27 15:49:40 PST 2012

Dear list and Hi Greg, 
In the recent raw2hdr bundle you packaged for Yulia http://www.radiance-online.org/pipermail/hdri/2012-February/000363.html there's a man page for hdrgen that list some options which do not appear in the man page that comes with the LINUX download you have on http://anyhere.com/

I have just discovered that the -x option does actually exist in the LINUX version of hdrgen, which is from around 2006, I think.

In the more recent (MacOS) version, the -x option is described as "-x Toggle overand under-exposed image removal. Normally "off," this option causes unnecessary exposures that are too light or too dark to contribute useful information to be automatically ignored."

I have always lived under the impression that 'useful information' is limited by pixel values of 200 in the darkest JPEG, and 20 (out of 255) in the brightest. I really do not remember where I took this from, but it must have been a post on the hdri list which I am unable to find now. Sorry.

In your message to hdri just recently, http://www.radiance-online.org/pipermail/hdri/2012-February/000365.html you stated "Specifically, all values in the short exposure's histogram should be below 245", which is different to the 200 threshold I mentioned above.

I have been experimenting with HDR photography for glare studies (think UGR), and have noticed some discrepancies in the results that one gets if hdrgen is run with and without the -x option. I was therefore wondering what hdrgen considers as "too light or too dark to contribute useful information". I would think that this is decision is made based on the value of the darkest/brightest pixel in the image. Is this assumption correct, and if so, what are the threshold values that are used with the -x option?

Kind regards

Axel

Gregory J. Ward gregoryjward at gmail.com Mon Feb 27 17:04:46 PST 2012

Hi Axel,

I tend not to use the -x option for critical work, as it's mostly a time-saver as opposed to a way to improve accuracy.

That said, the value range I consider "safe" extends from 27 to 228 in the 8-bit domain. I have found this empirically to be above the noise floor (provided the ISO setting is not too high) and below where some cameras introduce highlight roll-off.

I cannot use a strict cut-off for image values. Up to $0.2 \%$ of the pixels below the minimum are ignored and $0.05 \%$ of pixels above the maximum, likewise. This avoids issues with stuck pixels, which would otherwise make the -x option useless. This is also why it is better not to use -x for critical work, because you may lose the peak highlight in your image if it happens to be very small. 
Note also that hdrgen would never discard an input exposure off the end because it was "in range." Only the exposures shorter than first one below the safe maximum and the exposures longer than last one above the safe minimum that are considered superfluous.

I have a Lubuntu installation running under VMWare with gec version 4.6.19ubuntu3. I could use it to recompile hdrgen if you like, but I'm not sure what machines it would run on....

Cheers,

-Greg

\section{March 2012}

HDRI capture of LED (histograms and range)

Tyukhova, Yulia ytyukhova at unomaha.edu

Thu Mar 1 12:46:29 PST 2012

Hello!

I've been experimenting with the number of photos to include in final HDRI.

I took a sequence of photos of a single LED with reflectance standards included in the scene.

EOS7D $28-105 \mathrm{~mm}$ lens at $28 \mathrm{~mm}$

F16 1/8000-1/15,'

F4 1/30-5 mins

With the ND filter $\mathrm{t}=0.0094$

Images are fused with raw $2 \mathrm{hdr}$.

They were calibrated at white reflectance standard $215 \mathrm{~cd} / \mathrm{m} 2$.

I've noticed the following tendency:

If I fuse different number of photos (cut the number of photos on the shortest end), after calibrating at white reflectance standard, I get different luminance values for the LED.

*Shortest exposure to fuse $\quad \mathrm{L}, \mathrm{cd} / \mathrm{m} 2 *$
$1 / 125$ ' f16
$4.5 * 106$
$1 / 250$ ' f16
$9.06 * 106$
$1 / 500$ ' f16
$18 * 106$

I've seen interesting discussion between Axel and Greg on photos to include. But it seems like there are many uncertainties.

In HDRI second edition book it says "The darkest exposure should have no RGB values greater than 200 or so, and the lightest exposure should have no RGB values less than 20 or so. Do NOT include an excess of exposures beyond this range, as it will do nothing to help with the response recovery and may hurt." 
I assume it is the same for any HDRI sequence, not only for response curve.

I have plenty of photos of dark exposures that have no values greater than 200, same with the light exposures and 20.If somebody can clarify what photos should be included or have any other suggestions that would be great

Thank you,

*Yulia*

Gregory J. Ward gregoryjward at gmail.com

Thu Mar 1 17:03:41 PST 2012

Hi Yulia,

Since your maximum values are tracking the differences in your shorter exposures, it seems to indicate that you still haven't captured the brightest point on the LED. What are you looking at that makes you think the exposures have nothing over 200 ?

There is no harm in the newer version of hdrgen in including exposures that are too dark or too light. More naive methods might have trouble with this, but not hdrgen or raw2hdr.

Best,

-Greg

Moeck, Dr. Martin m.moeck at osram.com

Fri Mar 2 04:04:56 PST 2012

I suggest to use two parallel approaches, one with your LED, one with an incandescent low voltage. The problem with LED luminance measurements from HDRI is that a) the color gamut is small and b) the photopic sensitivity curve $\mathrm{V}$ (lambda) currently used is incorrect. It underestimates blue wavelengths quite a bit. Therefore, start with an incandescent first, because LEDs have a strong blue peak around 440 or $460 \mathrm{~nm}$, depending on the manufacturer. You are dealing with the liumitations of $\mathrm{V}$ (lambda) and then with tristimulus values which are just not good enough for narrowband LEDs, including white ones with a blue peak.

When you use the incandescent instead use it in a black lab. Place a few white paper samples on the walls at different known locations. Place your incandescent at a defined location. Derive the geometry, all distances and all angles between those white papers and the lamp. Turn the incandescent on and the room lights off. Measure the luminance of the white papers at a location that you marked with a soft pencil. Make HDR images and determine the luminance and scale.

Based on the luminance values of the papers, you can now derive the luminance of the incandescent filament. Make HDR images of the filament as seen from those papers. Check if the corresponding illuminance values of the white paper at reflectance around $85 \%$ corresponds to the luminance values of the filament in that direction.

Repeat several times to give you confidence. 
Once you are heading in the right direction, you can repeat the whole procedure with an LED. But this time your values will be around 20-30\% off. You should really use a CCD camera with a photopic filter to get better values, and then try to come up with a procedure to minimize errors for the HDR approach.

Regards

Martin Moeck

Osram

Tyukhova, Yulia ytyukhova at unomaha.edu

Fri Mar 2 09:14:15 PST 2012

Hello everybody!

I appreciate your help and responses.

Greg,

I've been checking histograms with CANON Digital Photo Professional. I was checking L channel, I guess I need to make sure RGB values are below 200 as well. Are there any suggestions on this issue?

Martin,

That is a very interesting suggestion! I guess I can see the difference between the measurements of two light sources.

> ..the photopic sensitivity curve V(lambda) currently used is incorrect.

It underestimates blue wavelengths quite a bit.

>> You should really use a CCD camera with a photopic filter to get better values..

You say that V(lambda) underestimates the values. But doesn't CCD camera's photopic filter have the same response curve? So, it would have the same mistake.

Lars,

>> Did you derive the response curve of your camera using another setup (not the directly visible LED) and reuse that camera response later on your LED captures? Or do you take the camera response from the same set of images (showing the LED in an otherwise dark room) that you are going to assemble into a HDR?

I've tried two approaches. The first one is to derive the response curve from the scene with very dark and bright areas with smooth gradients and neutral colors. Then fuse them to obtain RC. And then I've used it in photosphere for subsequent HDRIs. And the second one is to fuse raw images with Greg's script raw2hdr, where you do not have to have response curve. Greg: "The raw2hdr script doesn't need to derive a response curve, since the sensor data is linear. Instead, it creates an output from dcraw that 
follows a 2.0 gamma and creates an artificial response curve of $x^{\wedge} 2$ to decode it. This reduces quantization errors from the 8-bit intermediate images". And then I can analyse my hdr image in Photosphere.

Gregory J. Ward gregoryjward at gmail.com

Fri Mar 2 09:25:58 PST 2012

Hi Yulia,

> I've been checking histograms with CANON Digital Photo Professional. I was checking L channel, I guess I need to make sure RGB values are below 200 as well. Are there any suggestions on this issue?

Photosphere or Photoshop can give you an RGB histogram (along with max. values) from an 8-bit image.

$>$ Martin,

$>$ That is a very interesting suggestion! I guess I can see the difference between the measurements of two light sources.

$>>>$..the photopic sensitivity curve V(lambda) currently used is incorrect. It underestimates blue wavelengths quite a bit.

> >> You should really use a CCD camera with a photopic filter to get better values.

$>$ You say that V(lambda) underestimates the values. But doesn't CCD camera's photopic filter have the same response curve? So, it would have the same mistake.

Typical cameras do not follow the CIE standard observer curves for a number of reasons. Instead, they use red, green and blue bandpass filters and a color matrix optimized for color reproduction using some set of patches. It's an under-constrained problem, and different makers will optimize their color transform matrix differently. This is why dcraw tends to be more reliable - Dave Coffin always derives his CTM the same way and doesn't bias it towards one set of colors or another.

Even so, Martin is correct that highly saturated colors, such as those produced by typical LEDs, will often cause problems for reproduction and luminance estimation. There is no easy fix for this, unfortunately.

Best,

-Greg

HDRI analysis

Tyukhova, Yulia ytyukhova at unomaha.edu

Sun Mar 11 22:52:40 PDT 2012

Hello everybody,

I have a couple of questions on HDR image analysis.

How could I extract the luminance values from image.hdr?

With the pvalue tool I get RGB values. And in August 2007 thread Greg mentioned this (to get $\mathrm{L}$ ):

$\%$ pvalue $-\mathrm{h}-\mathrm{H}-\mathrm{b}$ render.pic $\backslash$ 


$$
\text { | rcalc }-\mathrm{e} ' \$ 1=\$ 1 ; \$ 2=\$ 2 ; \$ 3=179 * \$ 3 '
$$

But I would assume this is for .pic files. Is there a way how I can get it from .hdr that was already calibrated in Photosphere?

How is a standard deviation calculated in Photosphere? What does 1.1x mean? I would expect to see number closer to the luminance value displayed in the image.

In June 2009 thread there was a discussion on averaging luminance values withing the circular area. From anybody's experience, what is the easiest way to get average $\mathrm{L}$ value from hdr image within the circle area?

Thank you for all your help,

*Yulia*

Gregory J. Ward gregoryjward at gmail.com

Sun Mar 11 23:38:49 PDT 2012

Hi Yulia,

You need the "-o" option to pvalue, and the rest should work. The ".pic" file is the same as the ".hdr" file produced by Photosphere, a.k.a. "Radiance RGBE format" in the "Save As..." dialog.

The standard deviation for luminance is computed in the log domain, so what it gives you is a multiplier/divisor on the value rather than an absolute deviation. In other words, one standard deviation above is $1.1 \mathrm{x}$ the median, and one standard deviation below is median/1.1.

Averaging in a circular area is tricky and usually not worth the effort. There aren't any luminance meters that are accurate enough about their circles to worry about the difference between a circle and a square. If you must do it, it's a long and nastylooking pcomb command.

Best,

-Greg 


\section{Appendix E: Radiance tools \\ (http://www.radiance-online.org/pipermail/hdri/2012-February/000363.html)}

\section{hdrgen}

Create a high dynamic-range image from multiple exposures of a static scene. The input files may be JPEG or TIFF, but must be 24-bit RGB (trichromatic) images. The output is your choice of a Radiance HDR picture or a 32-bit LogLuv TIFF image. The syntax of the hdrgen command is:

hdrgen $-\mathrm{o}$ out_file $[-\mathrm{r}$ cam.rsp $][-\mathrm{m}$ cachesiz $][-\mathrm{a}][-\mathrm{e}][-\mathrm{s}$ stonits 1$]$ image1 $[-$ s stonits2] image2 ...

As many exposures may be given as necessary, and should ideally be spaced within two f-stops of each other. The brightest exposure should have no black pixels, and the darkest exposure should have no white pixels, but there is little point in extending beyond these limits, which may cause problems in determining the camera response function. The order of options and input files is unimportant, with the exception of the - s option, which must preceed the corresponding exposure. Following is an explanation of the options and their meanings:

-o out_file

Write high dynamic-range image to the given file. If the file has a '.tif' suffix, it will be written out as a LogLuv TIFF image. If it has a '.exr' suffix, it will be written out as an ILM OpenEXR image. If it has a '.jpg' suffix, it will be written out in JPEGHDR format. If it has any other suffix or none at all, it will be written out as an RLE RGBE Radiance picture. If the file exists, it will not be overwritten unless the '-F' option is specified.

-F Toggle output file overwrite switch (defaults to "false").

-k var_file

Write variance image to the given file, using the same format rules as the output file. This image indicates where the input deviates from an ideal exposure sequence, and may be useful for diagnostic purposes or further image processing.

-q quality

Set output quality to quality (0-100). This affects the JPEG output compression, and potentially the details of the other formats written as well. (For example, writing out a TIFF with -q 100 results in a 96-bit/pixel IEEE floating-point file rather than a LogLuv encoding.)

-r cam.rsp

Use the given file for the camera's response curves. If this file exists, it must contain the coefficients of three polynomials, one for each color primary. If the file does not exist, hdrgen will use its principal algorithm to derive these coefficients and write them out to this file for later use. If a scene contains no low frequency content or gradations of intensity, it may be impossible to derive the response curve from the exposure sequence. Thus it is better to create this information once for a given camera and reuse it for other sequences.

-c $C S$

Specify the output color space, where $C S$ is one of "sRGB" for standard CCIR709 primaries (the default), "XYZ" for CIE XYZ space, or "AdobeRGB" for Adobe RGB color space. Note that XYZ output will only be preserved in the TIF LogLuv and Radiance output formats.

-m cachesiz

Specify the cache size to use in megabytes. No more than this much memory will be allocated to hold image data during processing. The default value is 100 . Using a 
smaller value may require longer processing if many input images are used, since some will need to be read in twice rather than once, but specifying a larger value than there is memory available will definitely be worse, due to virtual memory swapping. -a Toggle automatic exposure alignment. The default value is "on," so giving this option one time switches it off. The alignment algorithm examines neighboring exposures and finds the pixel offset in $\mathrm{x}$ and $\mathrm{y}$ that minimizes the difference in the two images. It may be necessary to switch this option off when dealing with very dark or very bright exposures taken in a tripod-stabilized sequence.

-e Toggle exposure adjustment. Normally "on," exposure adjustment fine-tunes the scale difference between adjacent images to account for slight inaccuracies in the aperture or speed settings of the camera.

-x Toggle over- and under-exposed image removal. Normally "off," this option causes unnecessary exposures that are too light or too dark to contribute useful information to be automatically ignored.

-f Toggle lens flare removal. Normally, "off," this option is designed to reduce the scattered light from a camera's lens and aperture, which results in a slightly fogged appearance in high dynamic-range images.

-g Toggle ghost removal. Normally "off," this option attempts to remove moving or changing objects in a scene, which cause ghosts in the combined output. -s stonits

Set the sample-to-nits $\left(\mathrm{cd} / \mathrm{m}^{2}\right)$ conversion factor for the following image to the floating-point value stonits. This is normally determined automatically by the program from camera information stored in the Exif image header. If the image did not come directly from a digital camera, then it will be necessary to use this option for each image. If the absolute conversion is unknown, then simply pick a value for the brightest image, and increase it subsequently for each exposure in the sequence. One f-stop requires doubling this conversion factor, and two f-stops requires quadrupling. Diagnostics

The primary failure mode for this algorithm is the one mentioned in the description of the -r option, when the exposures contain too little information to solve for the camera response function. The best solution to this problem is to take off the exposures that are very light and very dark, or to use a different sequence of images to generate a response file. This file may then be used to combine the entire set of images, since the program no longer needs to solve for the responses.

Most of the other diagnostics you will encounter are "warnings," which means that the final image will be written, but may have problems. In particular, when the alignment algorithm fails on a hand-held sequence, some ghosting may be visible on high contrast edges in the output. Using the -a option to turn off automatic alignment will eliminate the warning, but unless the sequence was taken on a very stable tripod, the results will usually be worse rather than better.

Example

To combine all JPEG images matching a given wildcard and put into a LogLuv TIFF: hdrgen P13351?.JPG - o testimg.tif

Author

This software was written by Greg Ward of Exponent Corporation. Send comments or questions to gward@exponent.com or gward@1mi.net.

References

Tomoo Mitsunaga and Shree Nayar, "Radiometric Self-Calibration," Proceedings of IEEE Conference on Computer Vision and Pattern Recognition, June, 1999. 
Greg Ward, "LogLuv encoding for full-gamut, high-dynamic range images ," Journal of Graphics Tools, 3(1):15-31 1998.

Greg Ward, High Dynamic Range Images, web page.

Paul Debevec, web page.

\section{deraw}

Updated: May 14, 2009

NAME

dcraw - command-line decoder for raw digital photos

SYNOPSIS

dcraw $[O P T I O N] \ldots[$ FILE $] \ldots$

DESCRIPTION

dcraw decodes raw photos, displays metadata, and extracts thumbnails.

GENERAL OPTIONS

$-\mathbf{v}$

Print verbose messages, not just warnings and errors.

$-\mathbf{c}$

Write decoded images or thumbnails to standard output.

-e

Extract the camera-generated thumbnail, not the raw image. You'll get either a JPEG or a PPM file, depending on the camera.

$-\mathbf{z}$

Change the access and modification times of an AVI, JPEG, TIFF or raw file to when the photo was taken, assuming that the camera clock was set to Universal Time.

$-\mathbf{i}$

Identify files but do not decode them. Exit status is 0 if dcraw can decode the last file, 1 if it can't. -i - v shows metadata.

dcraw cannot decode JPEG files!!

\section{REPAIR OPTIONS}

-P deadpixels.txt

Read the dead pixel list from this file instead of ".badpixels". See FILES for a description of the format.

-K darkframe.pgm

Subtract a dark frame from the raw data. To generate a dark frame, shoot a raw photo with no light and do deraw $-\mathbf{D} \mathbf{- 4}-\mathbf{j} \mathbf{- t} \mathbf{0}$.

\section{-k darkness}

When shadows appear foggy, you need to raise the darkness level. To measure this, apply pamsumm -mean to the dark frame generated above.

\section{-S saturation}

When highlights appear pink, you need to lower the saturation level. To measure this, take a picture of something shiny and do dcraw -D -4 -j -c photo.raw | pamsumm -

\section{max}

The default darkness and saturation are usually correct.

\section{-n noise_threshold}

Use wavelets to erase noise while preserving real detail. The best threshold should be somewhere between 100 and 1000 .

\section{-C red_mag blue_mag}

Enlarge the raw red and blue layers by the given factors, typically 0.999 to 1.001 , to correct chromatic aberration. 
$-\mathbf{H} 0$

Clip all highlights to solid white (default).

-H 1

Leave highlights unclipped in various shades of pink.

$-\mathrm{H} 2$

Blend clipped and unclipped values together for a gradual fade to white.

-H 3+

Reconstruct highlights. Low numbers favor whites; high numbers favor colors. Try $\mathbf{H} 5$ as a compromise. If that's not good enough, do -H 9, cut out the non-white highlights, and paste them into an image generated with $\mathbf{- H} \mathbf{3}$.

\section{COLOR OPTIONS}

By default, dcraw uses a fixed white balance based on a color chart illuminated with a standard D65 lamp.

$-\mathbf{w}$

Use the white balance specified by the camera. If this is not found, print a warning and use another method.

-a

Calculate the white balance by averaging the entire image.

-A left top width height

Calculate the white balance by averaging a rectangular area. First do dcraw -j -t $\mathbf{0}$ and select an area of neutral grey color.

-r mul0 mul1 mul2 mul3

Specify your own raw white balance. These multipliers can be cut and pasted from the output of dcraw -v.

$+\mathrm{M}$ or $-\mathrm{M}$

Use (or do not use) any color matrix from the camera metadata. The default is $+\mathbf{M}$ if $\mathbf{w}$ is set, $-\mathbf{M}$ otherwise. This option only affects Olympus, Leaf, and Phase One cameras.

$-0[0-5]$

Select the output colorspace when the -p option is not used:

0 Raw color (unique to each camera)

1 sRGB D65 (default)

2 Adobe RGB (1998) D65

3 Wide Gamut RGB D65

4 Kodak ProPhoto RGB D65

5 XYZ

-p camera.icm [ -o output.icm ]

Use ICC profiles to define the camera's raw colorspace and the desired output colorspace (sRGB by default).

-p embed

Use the ICC profile embedded in the raw photo.

INTERPOLATION OPTIONS

-d

Show the raw data as a greyscale image with no interpolation. Good for photographing black-and-white documents.

-D

Same as -d, but totally raw (no color scaling).

-h 
Output a half-size color image. Twice as fast as $\mathbf{- q} \mathbf{0}$.

$-\mathbf{q}$

Use high-speed, low-quality bilinear interpolation.

$-\mathbf{q} 1$

Use Variable Number of Gradients (VNG) interpolation.

$-\mathbf{q} 2$

Use Patterned Pixel Grouping (PPG) interpolation.

$-\mathbf{q} 3$

Use Adaptive Homogeneity-Directed (AHD) interpolation.

-f

Interpolate RGB as four colors. Use this if the output shows false $2 \times 2$ meshes with VNG or mazes with AHD.

-m number_of_passes

After interpolation, clean up color artifacts by repeatedly applying a 3x3 median filter to the R-G and B-G channels.

\section{OUTPUT OPTIONS}

By default, dcraw writes PGM/PPM/PAM with 8-bit samples, a BT.709 gamma curve, a histogram-based white level, and no metadata.

-W

Use a fixed white level, ignoring the image histogram.

\section{-b brightness}

Divide the white level by this number, 1.0 by default.

\section{-g power toe_slope}

Set the gamma curve, by default BT.709 (-g 2.222 4.5). If you prefer sRGB gamma, use -g 2.4 12.92. For a simple power curve, set the toe slope to zero.

$-6$

Write sixteen bits per sample instead of eight.

$-4$

Linear 16-bit, same as -6 -W -g 11.

$-\mathbf{T}$

Write TIFF with metadata instead of PGM/PPM/PAM.

-t [0-7,90,180,270]

Flip the output image. By default, dcraw applies the flip specified by the camera. -t $\mathbf{0}$ disables all flipping.

$-\mathbf{j}$

For Fuji Super CCD cameras, show the image tilted 45 degrees. For cameras with non-square pixels, do not stretch the image to its correct aspect ratio. In any case, this option guarantees that each output pixel corresponds to one raw pixel.

-s [0..N-1] or -s all

If a file contains $\mathrm{N}$ raw images, choose one or "all" to decode. For example,

Fuji Super CCD SR cameras generate a second image underexposed four stops to show detail in the highlights.

\section{FILES}

../.badpixels, ../.badpixels, ../../.badpixels, ...

List of your camera's dead pixels, so that dcraw can interpolate around them. Each line specifies the column, row, and UNIX time of death for one pixel. For example:

962911028350000 \# died between August 1 and 4, 2002

128510670 \# do not know when this pixel died 
These coordinates are before any cropping or rotation, so use dcraw - $\mathbf{j}$-t $\mathbf{0}$ to locate dead pixels.

AUTHOR

Written by David Coffin, dcoffin a cybercom o net

\section{PVALUE(1)}

NAME

pvalue - convert RADIANCE picture to/from alternate formats

\section{SYNOPSIS}

pvalue [ options ][ file ]

pvalue -r [ options ][ file1 [ file2 file3 ]]

\section{DESCRIPTION}

Pvalue converts the pixels of a RADIANCE picture to or from another format. In the default mode, pixels are sent to the standard output, one per line, in the following ascii format:

xpos ypos red green blue

If no file is given, the standard input is read.

The reverse conversion option (-r) may be used with a single input file or when reading from the standard input, but if the second form is used with three separate input files, the three primaries are presumed to be separated in these files.

-u Print only unique values in the output, skipping runs of equal pixels. Specifying $+u$ turns this option off, which is the default.

-o Print original values, before exposure compensation. If the input file is in XYZE format, the $Y$ (green) channel will correspond to units of $\mathrm{cd} / \mathrm{m}^{2}$. Otherwise, the RGB values should be in spectral $\mathrm{W} / \mathrm{steradian} / \mathrm{m}^{2}$. Specifying to uses final exposed values, which is the default.

-h Do not print header. Specifying $+h$ causes the header to be printed, which is the default.

-H Do not print the resolution string. (See also the -r option below.) Specifying an input resolution for reverse conversion also turns this option off. Specifying $+\mathrm{H}$ causes the resolution string to be printed, which is the default.

-s nbytes

Skip the specified number of bytes on the input header. This option is useful for skipping unintelligible headers in foreign file formats. (Does not work when reading from standard input.)

-e exposure

Adjust the exposure by the amount specified. If the exposure is being given as a conversion factor, use +e instead, so an EXPOSURE line will not be recorded in the header (if any).

-g gamma

Set gamma correction for conversion. When converting from a RADIANCE picture to another format, the inverse gamma is applied to correct for monitor response. When converting to a RADIANCE picture (-r option), the gamma is applied directly 
to recover the linear values. By default, gamma is set to 1.0 , meaning no gamma correction is performed.

-d Data only, do not print $\mathrm{x}$ and y pixel position.

-da Same as -d.

-di Print ascii integer values from 0 to $255+$. If + di is given, the integer values will be preceded by the $\mathrm{x}$ and $\mathrm{y}$ pixel locations.

-db Output binary byte values from 0 to 255 .

-dw Output binary 16-bit words from 0 to 65535.

-dW Output binary 16-bit words from 0 to 65535, byte-swapped.

-df Output binary float values.

-dF Output byte-swapped binary float values.

-dd Output binary double values.

-dD Output byte-swapped binary double values.

$-\mathrm{R}$ Reverse ordering of colors so that the output is blue then green then red. The default ordering (specified with $+\mathrm{R}$ )is red then green then blue.

-n The RGB values are non-interleaved, meaning that all the red, green and blue data are stored together in separate chunks. Interleaving may be turned on with the $+n$ option, which is the default.

-b Print brightness values rather than RGB. Specifying $+b$ turns this option off, which is the default.

-pP Put out only the primary $\mathrm{P}$, where $\mathrm{P}$ is one of upper or lower case $\mathrm{aRa}, \mathrm{aGa}$ or aBa for red, green or blue, respectively. This option may be used to separate the Radiance primaries into three files with three separate runs of pvalue, or only one file when only one primary is needed. Note that there is no space between this option and its argument.

-r Perform reverse conversion. Input is in the format given by the other options. The $\mathrm{x}$ and $\mathrm{y}$ resolution must be specified on the command line, unless the image file contains a Radiance resolution string at the beginning (see $-\mathrm{H}$ option above and $-\mathrm{y}$ option below). Specifying $+r$ converts from a Radiance picture to other values, which is the default.

-p xr yr xg yg xb yb xw yw

On reverse conversion, RGB input uses the given set of color primaries. These are written into the output header with the PRIMARIES variable.

-pXYZ On reverse conversion, input is in CIE XYZ coordinates, rather than RGB. The $Y$ channel is assumed to be in $\mathrm{cd} / \mathrm{m}^{2}$.

$-\mathrm{y}$ res Set the output $\mathrm{y}$ resolution to res. If $+\mathrm{y}$ is specified, then the scanlines are assumed to be in increasing order (i.e. bottom to top). The default value for this option is 0 , which means that the picture size and scanline order must appear as the first line after the header (if any) in the input file. Either an upper or lower case aYa may be used for this option. Since Radiance files always contain such a line, this option has no effect for forward conversions.

$+x$ res Set the output $x$ resolution to res. If $-x$ is specified, then the scanlines are assumed to be in decreasing order (i.e. right to left). The ordering of the $-y$ and $+x$ options determines whether the scanlines are sorted along $\mathrm{x}$ or along y. Most Radiance pictures are sorted top to bottom, then left to right. This corresponds to a specification of the form "-y yres +x xres". Either an upper or lower case aXa may be used for this option. Like the -y option, -x options have no effect for forward conversions. 


\section{EXAMPLE}

To look at the original, unique pixels in picture:

pvalue -o -u picture $\mid$ more

To convert from a 512x400 8-bit greyscale image in bottom to top, left to right scanline ordering:

pvalue $-\mathrm{r}-\mathrm{db}-\mathrm{b}-\mathrm{h}+\mathrm{y} 400+\mathrm{x} 512$ input.im > flipped.hdr

pdlip - $v$ flipped.hdr $>$ final.hdr

AUTHOR

Greg Ward

BUGS

The $-r$ option does not work with the -u option. Also, input pixel locations are ignored during a reverse conversion, so this information is not used in determining the scanline ordering or picture size. 


\section{Appendix F: HDRI of incandescent lamp}

HDRI's ability to capture an incandescent light source is tested (RAW images fused with raw2hdr).
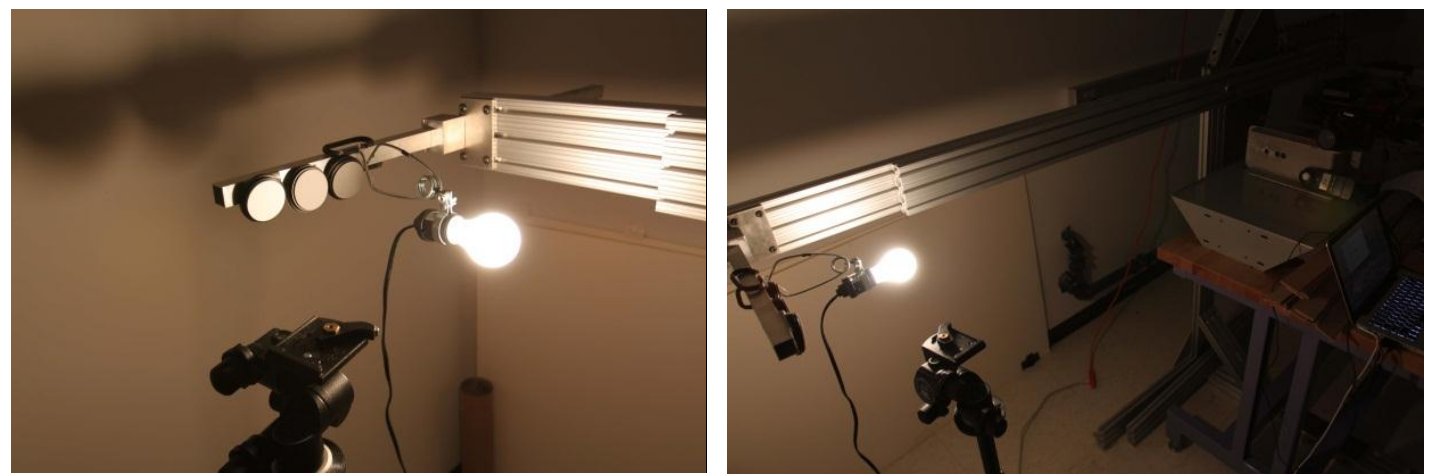

Figure F1. Experimental setting for making HDRI of the incandescent light source

Equipment and settings:

Lamp Sylvania 75W soft white 120V (A19, diameter $23 / 8$ ' = $60 \mathrm{~mm}$ );

Canon EOS 7D fitted with Canon zoom lens 28-105mm;

Aperture size f/8;

Shutter speeds $1 / 8000-8$ ',

Images are fused with raw2hdr Perl script. Luminances of reflectance standards and a particular part of the incandescent source are measured with LS110. After calibrating at a white reflectance standard $\left(350.2 \mathrm{~cd} / \mathrm{m}^{2}\right)$, HDRI's luminance measurements are compared with the luminance meter measurements (table F).

Table F. Luminance value measurements with the luminance meter compared to HDRI

\begin{tabular}{|c|c|c|c|c|}
\hline$\rho, \%$ & 20 & 40 & 99 & $\begin{array}{c}\text { Incandescent } \\
\text { lamp }\end{array}$ \\
\hline $\mathbf{L}_{\text {meter, }} \mathrm{cd} / \mathrm{m}^{2}$ & 64.05 & 166.2 & 350.2 & 32950 \\
\hline $\begin{array}{c}\mathbf{L}_{\text {HDRI } 28 \text { mm }} \\
\mathrm{cd} / \mathrm{m}^{2}\end{array}$ & 71.5 & 170 & $\begin{array}{c}\text { Calibrated at } \\
\text { this point }\end{array}$ & 33400 \\
\hline $\begin{array}{c}\mathbf{L}_{\text {HDRI } 105 \mathrm{~mm}}, \\
\mathrm{~cd} / \mathrm{m}^{2}\end{array}$ & - & - & - & 32500 \\
\hline \multirow[t]{2}{*}{ Error, \% } & \multirow[t]{2}{*}{11.6} & \multirow[t]{2}{*}{2} & \multirow[t]{2}{*}{0} & $\begin{array}{c}\text { Zoom } 28 \mathrm{~mm} \\
1.4 \%\end{array}$ \\
\hline & & & & $\begin{array}{c}\text { Zoom } 105 \mathrm{~mm} \\
1.4 \%\end{array}$ \\
\hline
\end{tabular}




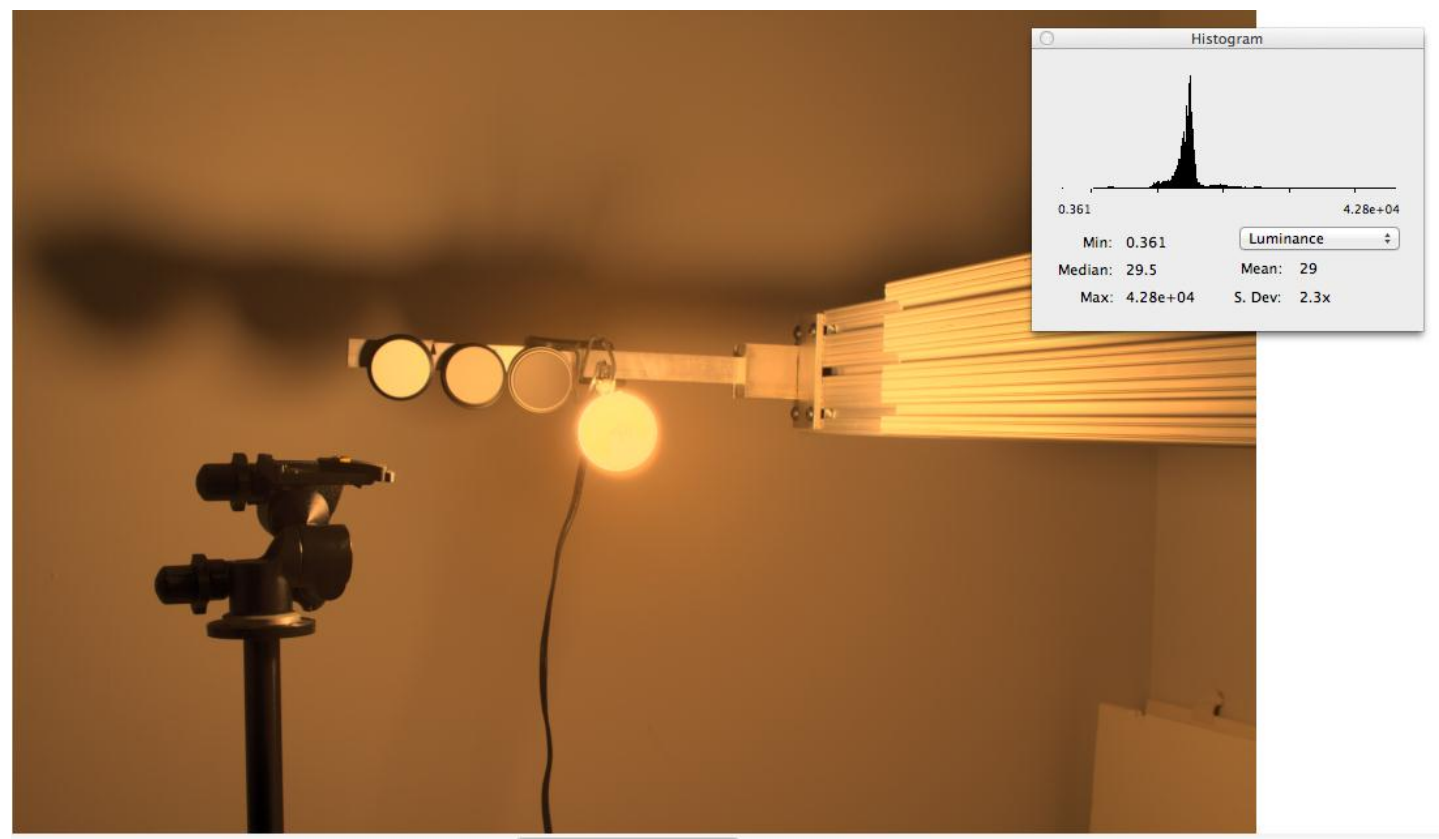

Figure F2. HDRI of incandescent light source captured with CANON EOS 7D fitted with $28-105 \mathrm{~mm}$ zoom lens at $28 \mathrm{~mm}$

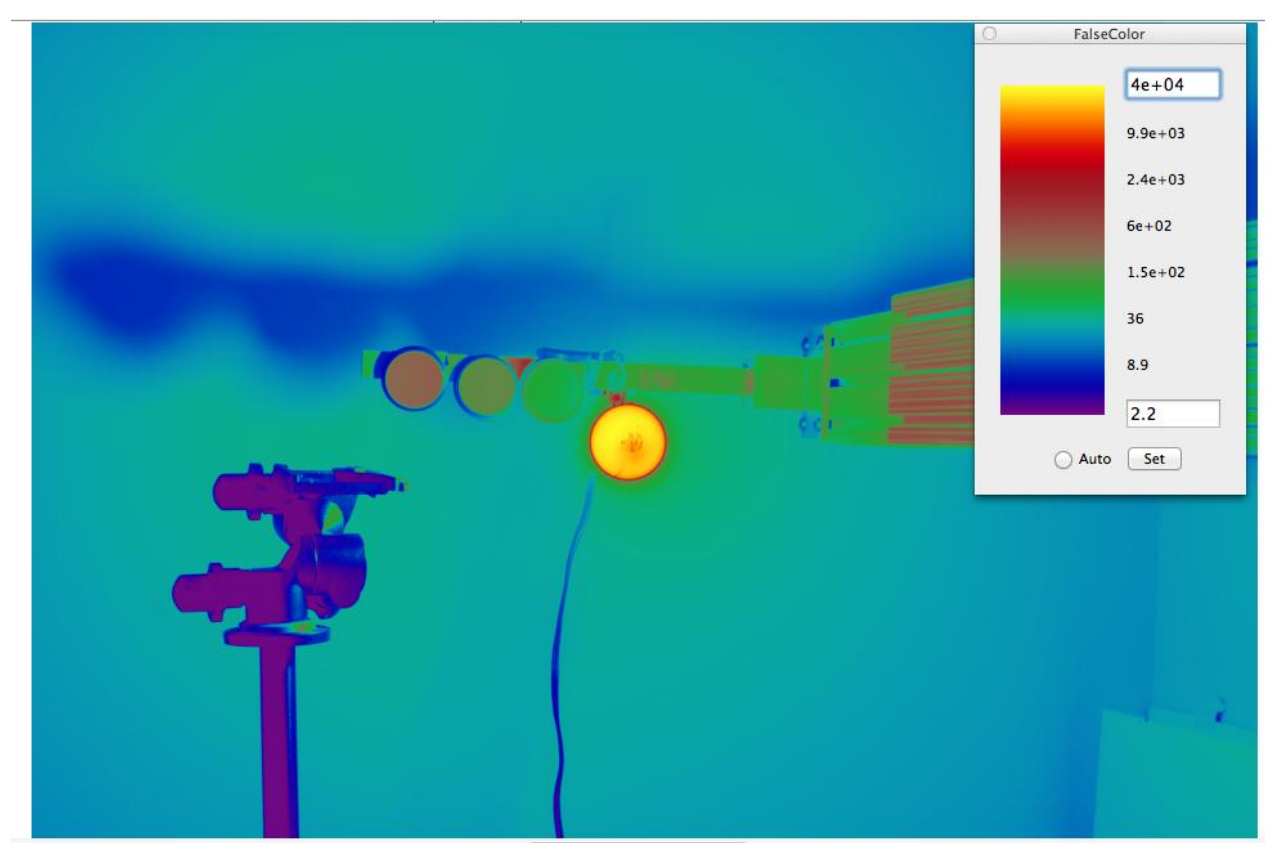

Figure F3. HDRI (pseudo colors) of incandescent light source captured with CANON EOS 7D fitted with $28-105 \mathrm{~mm}$ zoom lens at $28 \mathrm{~mm}$ 


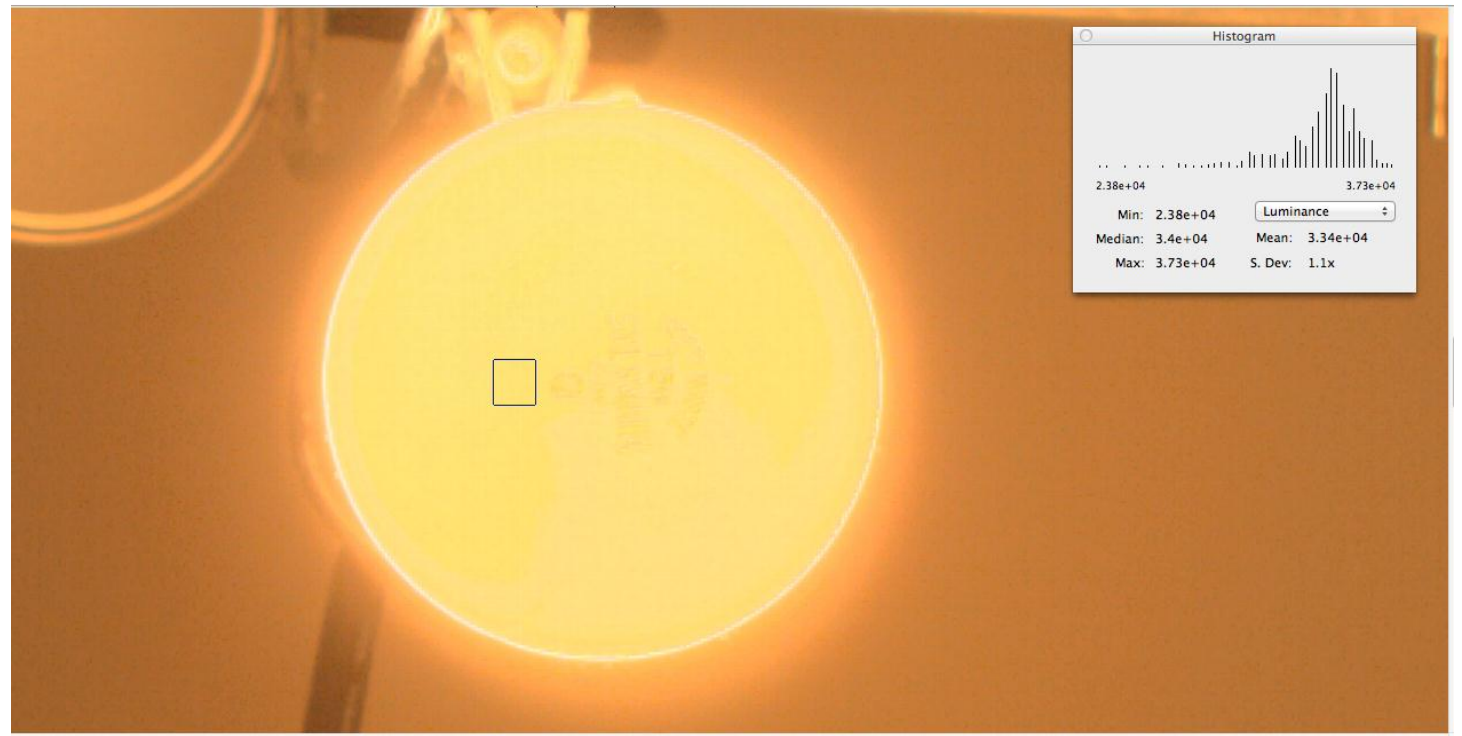

Figure F4. Enlarged HDRI of incandescent light source captured with CANON EOS 7D fitted with $28-105 \mathrm{~mm}$ zoom lens at $28 \mathrm{~mm}$

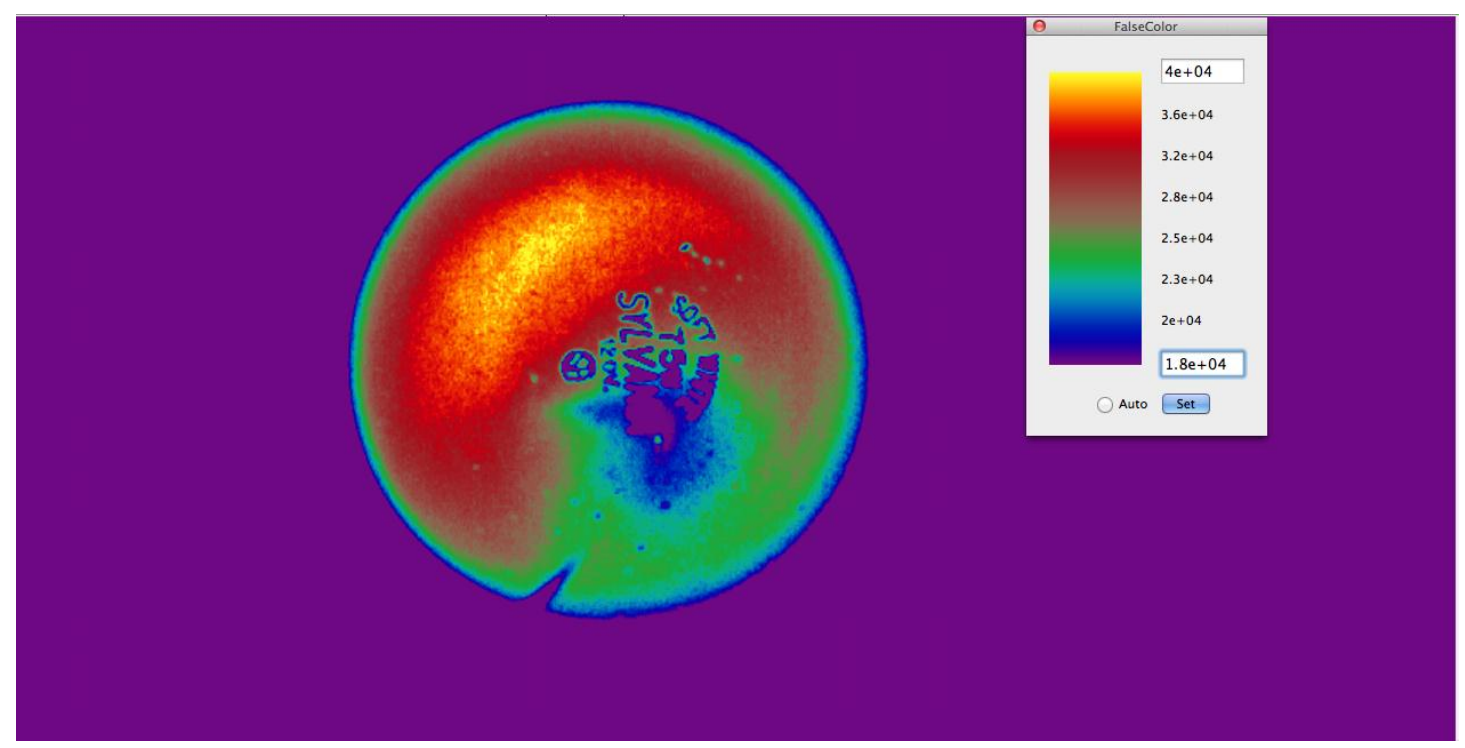

Figure F5. Enlarged HDRI (pseudo colors) of incandescent light source captured with CANON EOS 7D fitted with $28-105 \mathrm{~mm}$ zoom lens at 28 $\mathbf{m m}$ 


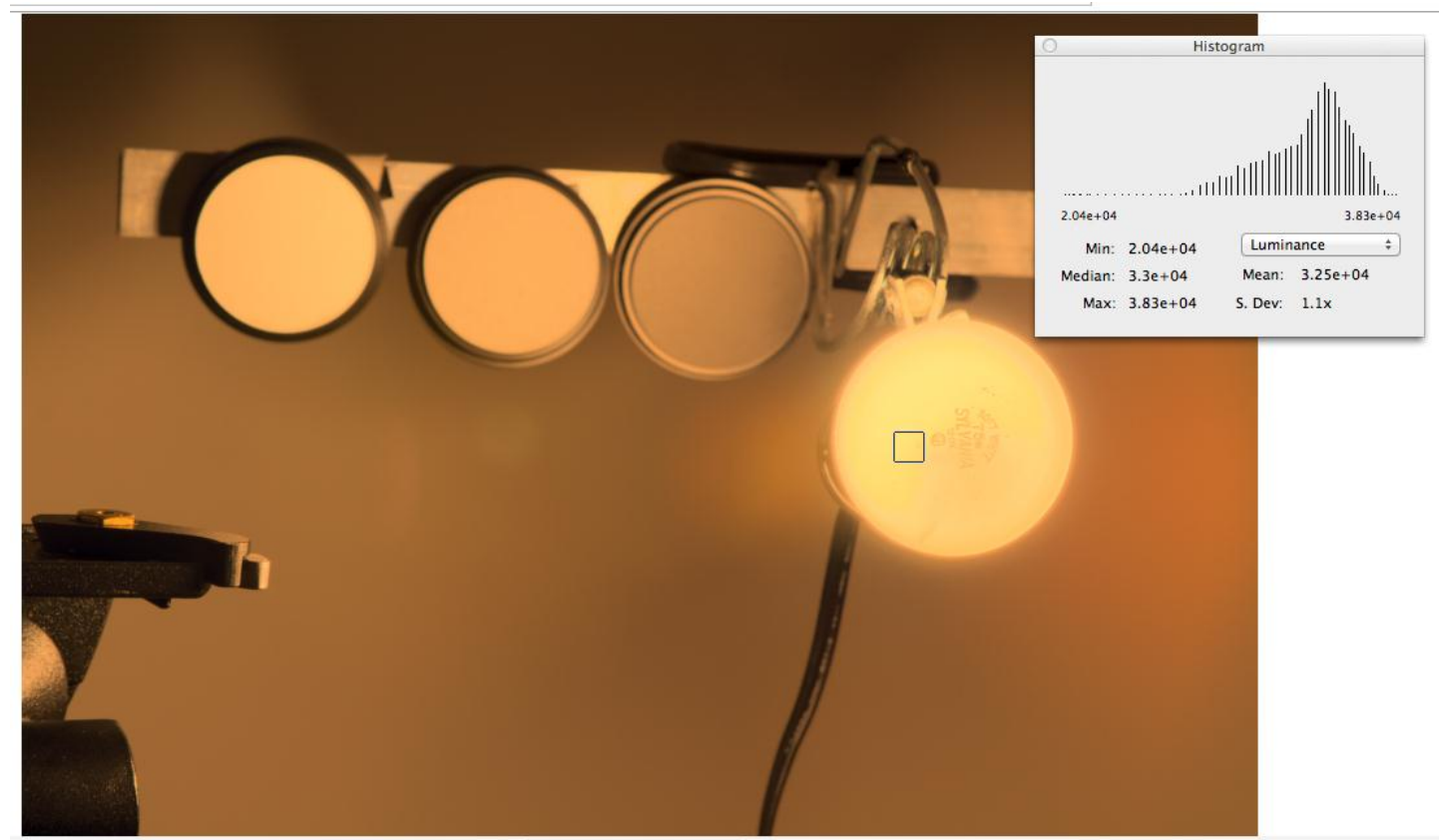

Figure F6. HDRI of incandescent light source captured with CANON EOS 7D fitted with $28-105 \mathrm{~mm}$ zoom lens at $105 \mathrm{~mm}$

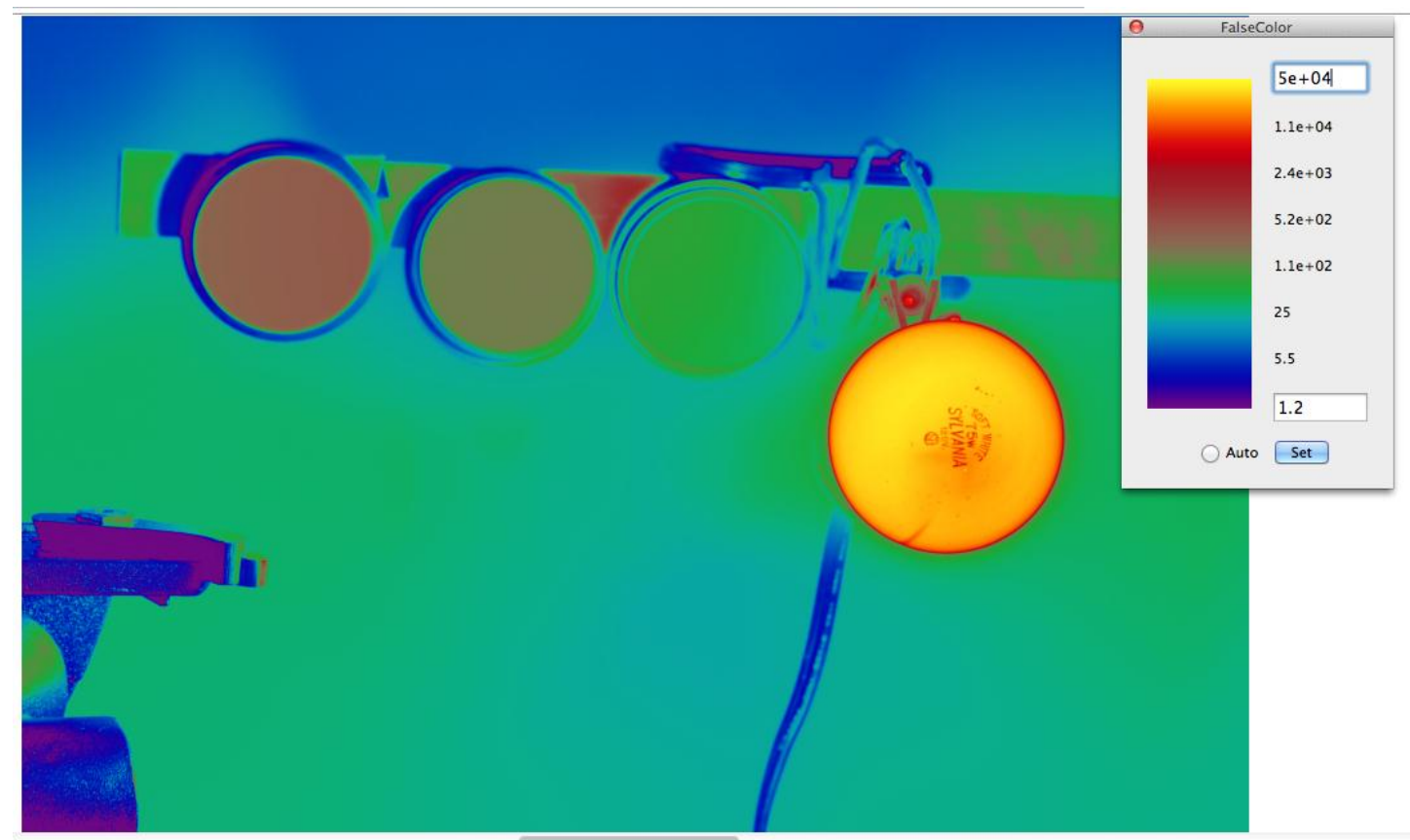

Figure F7. HDRI (pseudo colors) of incandescent light source captured with CANON EOS 7D fitted with $28-105 \mathrm{~mm}$ zoom lens at $105 \mathrm{~mm}$ 


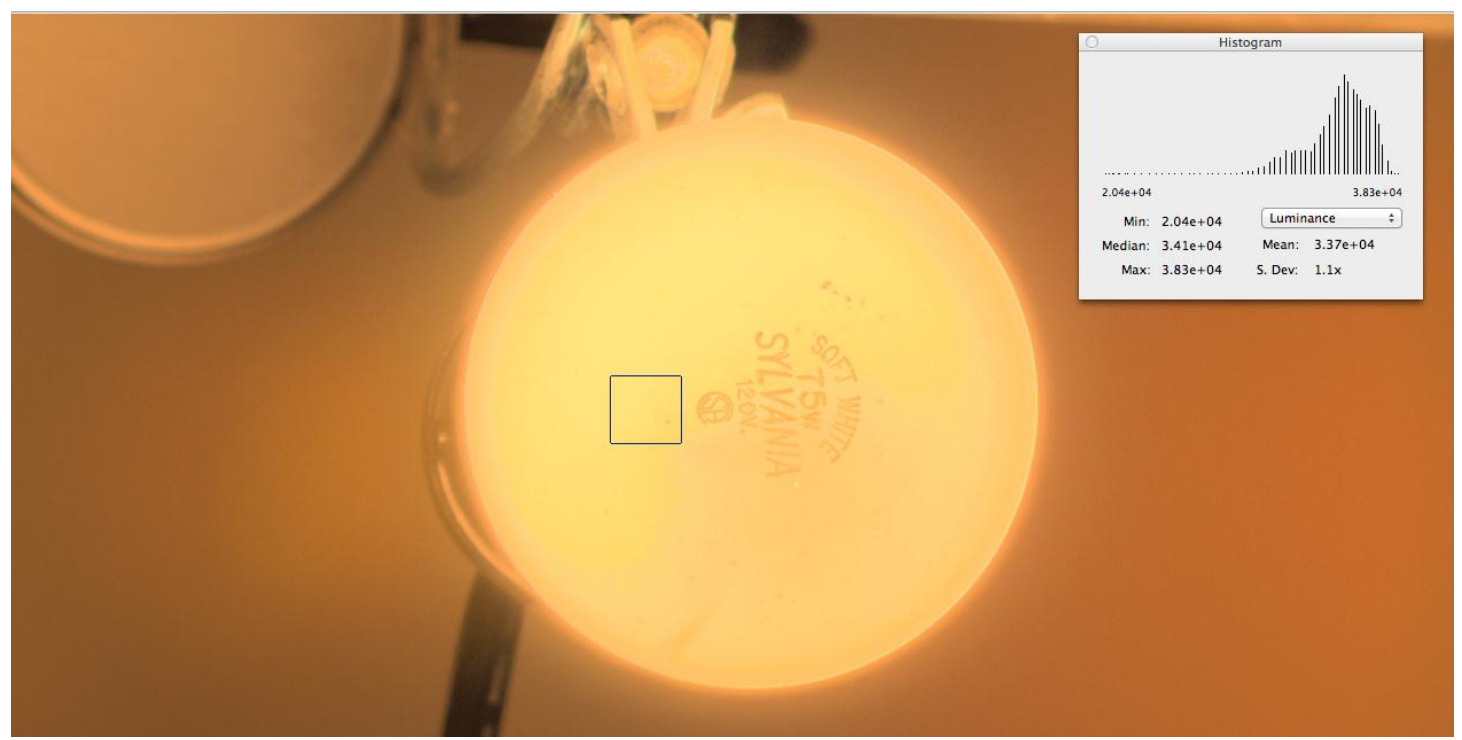

Figure F8. Enlarged HDRI of incandescent light source captured with CANON EOS 7D fitted with $28-105 \mathrm{~mm}$ zoom lens at $105 \mathrm{~mm}$

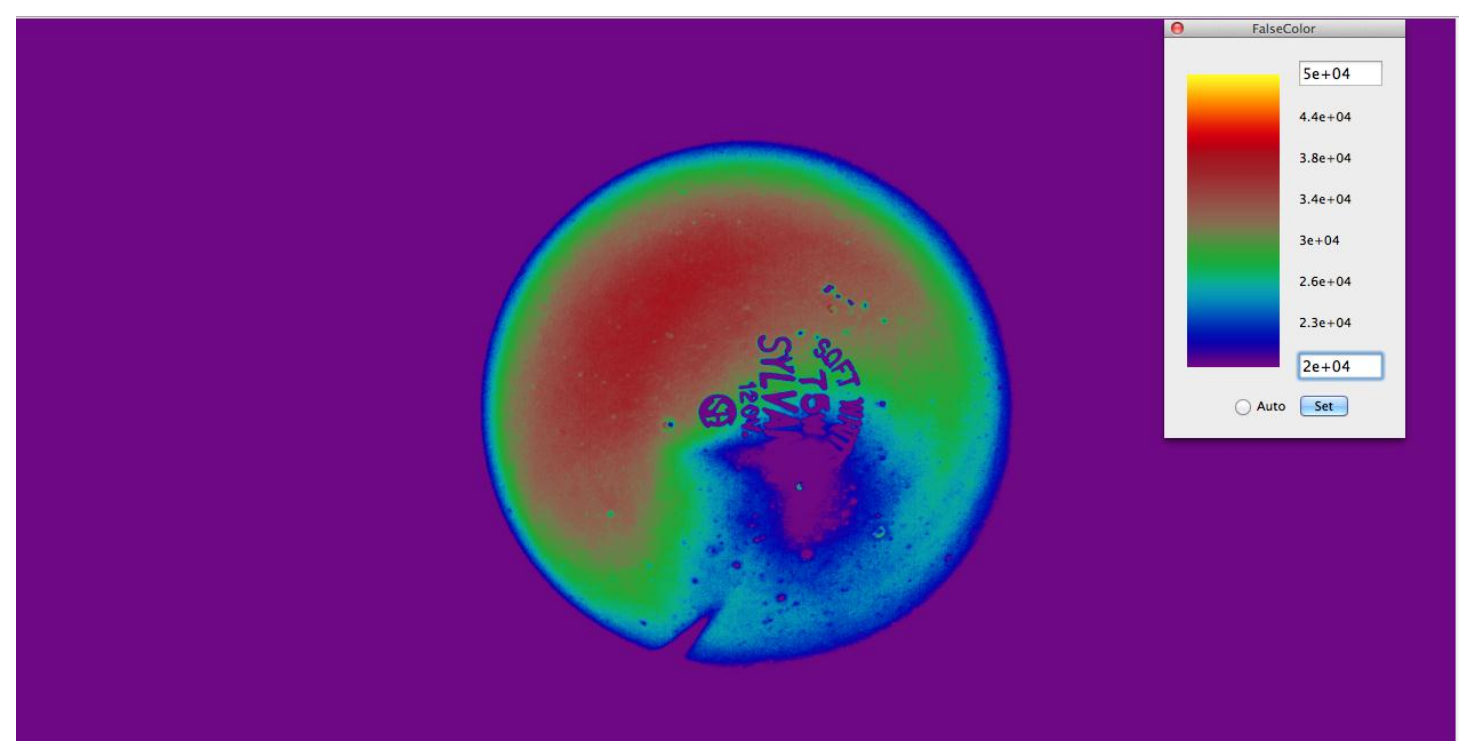

Figure F9. Enlarged HDRI (pseudo colors) of incandescent light source captured with CANON EOS 7D fitted with $28-105 \mathrm{~mm}$ zoom lens at 105 $\mathbf{m m}$

Outcome: HDRI's luminances of an incandescent lamp are within the acceptable ranges (1.4\% error). It is tested for a particular camera/lens combination for both zoom conditions ( 28 and $105 \mathrm{~mm}$ ) compared to the reference luminance measurement obtained with LS110. 